Search for the Tô Quark in

\title{
UTPP 42
}

\section{8-TeV Proton-Antíproton Gollisions}
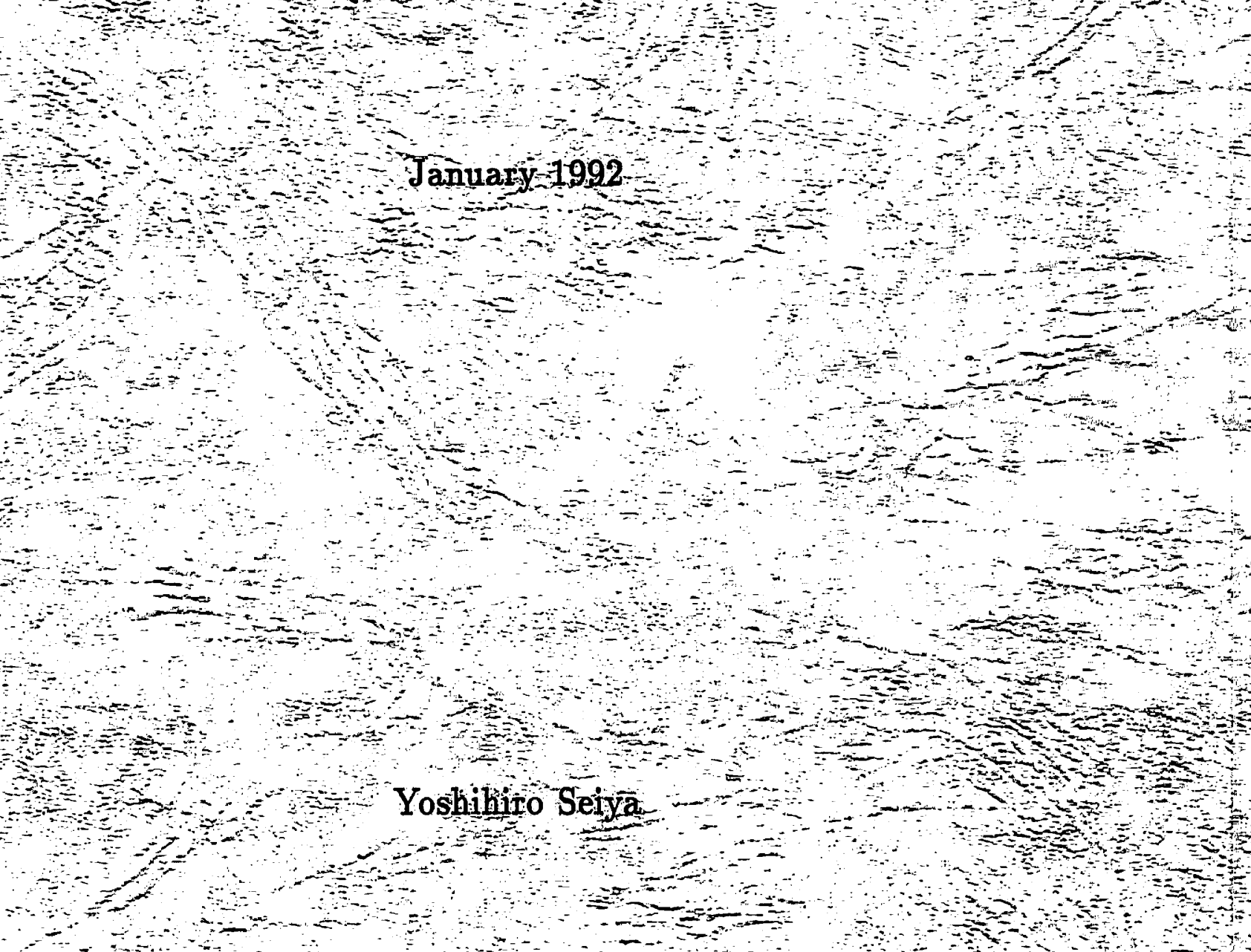

Submited in partial fulfiliment of the requirements $f$

the Degree of Doctor of Science in. the Doctoral program in

University of Tsukuba

3
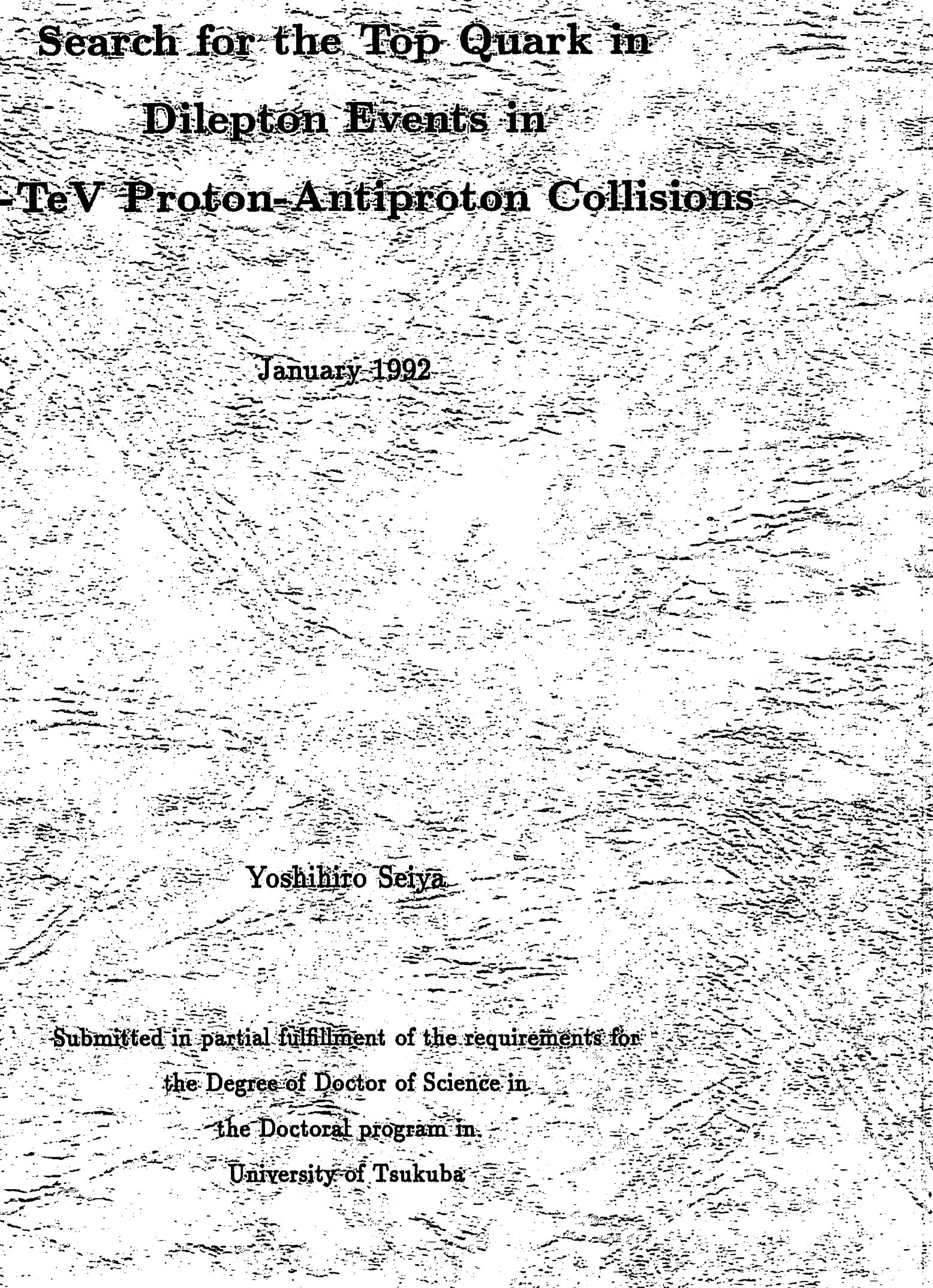

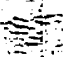 20 satisis:

\section{Dilepton Events in}

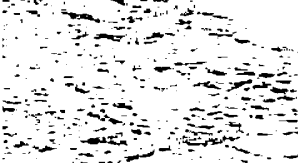




\title{
Search for the Top Quark in
}

Dilepton Events in

\section{8-TeV Proton-Antiproton Collisions}

\author{
January 1992
}

Yoshihiro Seiya

Submitted in partial fulfillment of the requirements for the Degree of Doctor of Science in the Doctoral program in University of Tsukuba 


\begin{abstract}
A search for the top quark in $p \bar{p}$ collisions at a center of mass energy of $1.8 \mathrm{TeV}$ is described. The data were collected with the Collider Detector at Fermilab (CDF) during the 1988-1989 Tevatron collider run. The corresponding integrated luminosity is 4.1 $\mathrm{pb}^{-1}$.

The Standard Model predicts the existence of a weak isospin partner of the bottom quark, called the top quark $(t)$. Although several experimental results suggest the existence of the top quark in the context of the Standard Model, it is yet to be observed. Previous experiments exclude the top quark with mass less than $77 \mathrm{GeV} / c^{2}$ at $95 \%$ confidence level.

The CDF detector is a general purpose detector built to explore $p \bar{p}$ collisions. It covers almost the full solid angle around the $p \bar{p}$ interaction point. The CDF consists of tracking detectors and calorimeters for measurements of the particle momentum and energy. Muon detectors are instrumented at the most outside of the detector. Using these detector components, the CDF is capable of identifying electrons, photons, muons, neutrinos (as the imbalance of the total transverse energy), hadrons and jets.

In the top quark search described in this thesis, the top quark production via the strong interaction process, $p \bar{p} \rightarrow t \bar{t}$, is considered because it dominates the top quark production at the Tevatron energy. After the production, the top quark decays into a $W$ and a bottom quark $b$ via the weak charged current decay assumed in the Standard Model. The $W$ suceedingly decays into a quark-antiquark pair or a lepton-neutrino pair. Since a dilepton channel where both the $t$ and $\bar{t}$ quark decay semileptonically,

$$
t \bar{t} \rightarrow\left(W^{+} b\right)\left(W^{-} \bar{b}\right) \rightarrow\left(l^{+} \nu b\right)\left(l^{\prime-} \bar{\nu}^{\prime} \bar{b}\right),
$$

provides the cleanest signature in $p \bar{p}$ collisions, we consider this dilepton channel for the top quark search.

We select events containing two leptons $(e \mu, e e$ and $\mu \mu)$ by exploiting the good electron and muon identification capabilities of the CDF detector. The main background processes to the $t \bar{t}$ dilepton events are the $b \bar{b}$ production and the Drell-Yan process. Considering the decay kinematics of the top quark with much heavier mass compared to the $b$ quark mass, it is shown that the $b \bar{b}$ background is effectively removed by requiring the leptons to have high momentum in the transverse plane (the high $P_{\mathrm{T}}$ cut) and to be isolated from other particle activities in the events (the isolation cut). Further cuts on some event kinematical and topological properties are imposed to reduce the dilepton background from the Drell-Yan process. Concretely, it is shown that the appropreate cuts on the opening angle of the two leptons in the transverse plane, the missing transverse energy (the imbalance of the transverse energy) and the invariant mass formed by the two leptons are effective to remove the Drell-Yan dilepton background. After applying all the selection requirements for the $t \bar{t}$ events, that is, the lepton identification criteria, the high $P_{\mathrm{T}}$ cut, the isolation cut, and the event-kinematical and topological cut, one event was found in the data.
\end{abstract}


Based on that one event was found, we derive an upper limit on the top quark production cross section within the framework of the minimal Standard Model (with one neutral Higgs boson). We translate the upper limit on the cross section to the lower limit on the top quark mass using the theoretical predictions for the production cross section as a function of the top quark mass. The detection efficiency for the $t \bar{t}$ events, which is necessary for calculating the upper limit on the production cross section, is determined by the Monte Carlo method combining the lepton identificaiton efficiency and the lepton trigger efficiency estimated using the CDF real data. As a result, a lower limit on the top quark mass,

$$
M_{\text {top }}>85 \mathrm{GeV} / c^{2} \quad(95 \% \text { C.L. }),
$$

is obtained from the study on the high $P_{\mathrm{T}}$ dilepton events.

In order to get more stringent mass limit, we combine another dilepton analysis performed by other CDF collaborators on an independent channel of the high $P_{\mathrm{T}}$ dilepton. The analysis searches for the $t \bar{t}$ events with a high $P_{\mathrm{T}}$ lepton from the top quark decay and a low $P_{\mathrm{T}}$ muon from the decay chain of $t \rightarrow b \rightarrow \mu$. From the inclusion of this channel, We obtain

$$
M_{\text {top }}>91 \mathrm{GeV} / c^{2} \quad \text { (95\% C.L.) }
$$

as a final result. Currently, this is the most stringent limit on the top quark mass available in the world. 


\section{Acknowledgements}

I want to thank my advisor, Professor Koji Takikawa, for his support throughout my graduate student career. I learned much from his precise way of thinking and serious attitude to physics research. I am also grateful for his careful reading of the text and his many criticisms to the point. I want to thank Professor Kunitaka Kondo who gave me the opportunity to work at CDF and a constant support throughout my graduate student career. It was a great fortune for me that $I$ had a chance to work with him and to share his keen insights early in my graduate years. His enthusiasm for physics has always encouraged me.

G. P. Yeh coordinated this analysis and provided me a lot of suggestions at crucial points. Milciades Contreras devoted much time with me to discussing about and checking out this analysis. I could not have finished this work without their continuous help. They have been always kind, thoughtful and gave encouragement to me not only as my collaborators of this work but also as my personal friends.

I am grateful to John Yoh, Paul Tipton and Claudio Campagnari for their support and discussions on the analysis. Many thanks go to Stefano Belforte, Bob Blair, Melissa Franklin and Lee Pondrom for their judicious criticisms, helpful suggestions and support to the analysis. Masanori Mishina, Yasuo Fukui, Yohei Morita and Howard Budd are appreciated for their help to my work on the detector. Conversations with Luc Demortier, Shuichi Kunori, Shoji Mikamo, Nobuaki Oshima, Taiji Yamanouchi, and Barbara Yeh made my stay at Fermilab unforgettable.

Discussions on physics and other various things with Shinghong Kim, Satoru Ogawa, Mikio Takano, Fumihiko Ukegawa, Mariko Ninomiya, Takashi Ino and Masahiko Yokoyama were very beneficial for me. I also wish to thank other members of the Tsukuba high energy physics group for their constant help. They include Fumio Abe, the late Yoshimi Funayama, Kazuhiko Hara, Hiroshi Iso, Shigeyuki Miyashita, Itsuo Nakano, Ryutaro Oishi, Kiyoshi Yasuoka, and other colleagues.

This thesis would not have been possible without the greatly intensive works of many 
CDF collaborators who have developed the detector and other analysis environment, the Fermilab accelerator people who constructed and operated the Tevatron with a remarkable success, the Fermilab technical staffs who made a great effort in the construction of the detector and the Tevatron, and the people of the Fermilab Computer Division who provided constant help for the computer work.

Finally, I want to express my great appreciation to Carol Picciolo, Kyoko Kunori, Kazuko Kumashiro and Mutsumi Uenishi for their help through their secretary works.

This work was supported by the Ministry of Science, Culture and Education of Japan, the Department of Energy, the National Science Foundation, Instituto Nazionale di Fisica Nucleare, and the A. P. Sloan Foundation. 


\section{Contents}

Acknowledgements $\quad$ i

List of Tables $\quad$ vi

List of Figures viii

The CDF Collaboration $\quad$ xv

1 Introduction 1

2 Top Quark in the Standard Model 5

2.1 Indication of the Existence of the Top Quark . . . . . . . . . 5

2.1.1 Phenomenological Evidences .............. 5

2.1 .2 Theoretical Indication $\ldots \ldots \ldots . \ldots . \ldots$

2.2 Constraints on the Top Quark Mass . . . . . . . . . . . . 9

2.2.1 Phenomenological Constraints .............. 9

2.2.2 Direct Searches for the Top Quark . . . . . . . . . . . 11

2.3 Hadronic Production of the Heavy Quark . . . . . . . . . . . . . . 14

2.4 Fragmentation of the Heavy Quark . . . . . . . . . . . . 17

2.5 Decay of the Heavy Quark . . . . . . . . . . . . . 18

2.6 Top Search in Dilepton Events at CDF . . . . . . . . . . . 19

3 The CDF Detector 31 
3.1 Detector Components . . . . . . . . . . . . . . . . . 31

3.1.1 Beam-Beam Counter . . . . . . . . . . . . . . 32

3.1 .2 Tracking Detector . . . . . . . . . . . . . 32

3.1.3 Calorimeter . . . . . . . . . . . . . 34

3.1.4 Central Muon Detector . . . . . . . . . . . . . . . 38

3.2 Trigger System $\ldots \ldots \ldots \ldots \ldots \ldots \ldots \ldots \ldots \ldots$

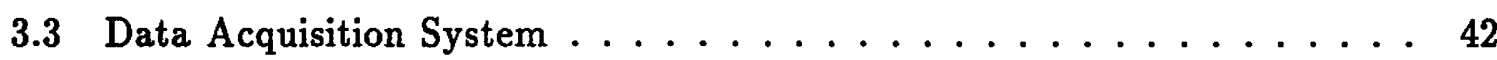

4 Event Trigger and Selection $\quad 55$

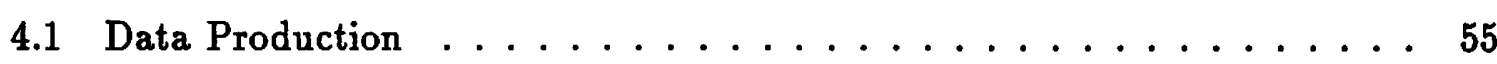

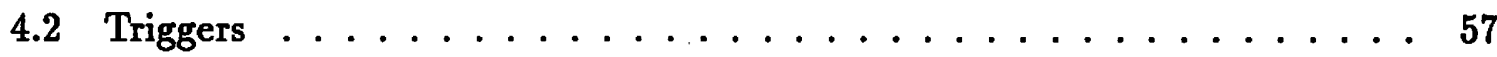

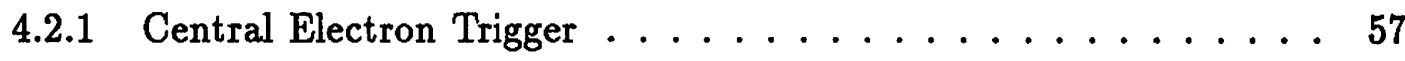

4.2 .2 Plug Electron Trigger . . . . . . . . . . . . . . . 59

4.2.3 Central Muon Trigger $\ldots \ldots \ldots \ldots \ldots \ldots$

4.2.4 Triggers for the Dilepton Events . . . . . . . . . . . . 60

4.3 Electron Selection . . . . . . . . . . . . . . . . 60

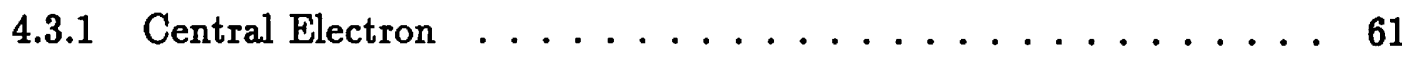

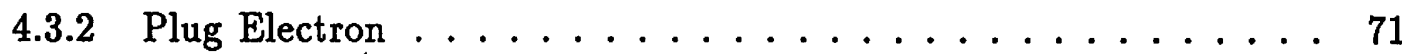

4.4 Central Muon Selection . . . . . . . . . . . . . . 76

4.5 Event Topology Cuts . . . . . . . . . . . . . . . . . . 79

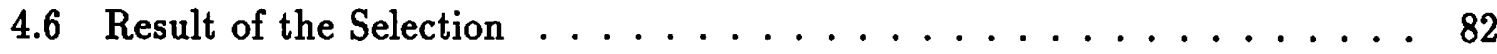

5 Detection Efficiency and Background 110

5.1 Detection Efficiency . . . . . . . . . . . . . . . . . 111

5.1.1 Monte Carlo Study . . . . . . . . . . . . . . . . . . . 112

5.1 .2 Lepton Identification Efficiency $\ldots \ldots \ldots \ldots \ldots$

5.1 .3 Trigger Efficiency . . . . . . . . . . . . . . . . . 122

5.1 .4 Summary . . . . . . . . . . . . . . . . . . . . 124

5.2 Systematic Uncertainty . . . . . . . . . . . . . . . 125 
5.3 Background Study . . . . . . . . . . . . . . . 129

6 Mass Limit on the Top Quark 159

$\begin{array}{llr}7 \text { Conclusions } & 167\end{array}$

$\begin{array}{ll}\text { A Luminosity Measurement } & 168\end{array}$

$\begin{array}{ll}\text { Bibliography } & 175\end{array}$ 


\section{List of Tables}

4.1 Triggers and corresponding dilepton event types which are selected by the

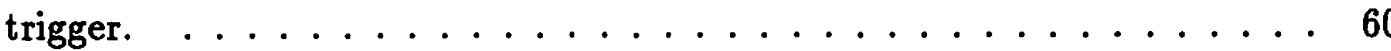

4.2 Characteristics of the top candidate event. The observed calorimeter $E_{\mathrm{T}}$ is used in the $P_{\mathrm{T}}$ column for the electron and jet clusters. . . . . . 83

5.1 The fiducial cut efficiencies for each type of leptons. For muons, the track quality cuts are included. The ISAJET Monte Carlo with $M_{\text {top }}=90$ $\mathrm{GeV} / \mathrm{c}^{2}$ was used to obtain the efficiencies. . . . . . . . . . . 114

5.2 The isolation cut efficiencies for each type of leptons from top quark of several masses. . . . . . . . . . . . . . . . . . . 115

5.3 The central electron identification cut efficiencies calculated from the CDF $Z \rightarrow e e$ and Monte Carlo $Z \rightarrow e e$ events. . . . . . . . . . 117

5.4 The plug electron identification cut efficiencies calculated from CDF $Z \rightarrow$ $e e$ and Monte Carlo $Z \rightarrow e e$ events. . . . . . . . . . . . . 120

5.5 The muon identification cut efficiencies calculated from CDF $Z \rightarrow \mu \mu$ and Monte Carlo $Z \rightarrow \mu \mu$ events. . . . . . . . . . . . . . 122

5.6 Dilepton detection efficiencies for $M_{\mathrm{top}}=90 \mathrm{GeV} / c^{2}$. The total dilepton efficiency at this mass is $\varepsilon_{\text {Total }}=(16.2 \pm 1.8) \%$. The efficiency $\varepsilon_{\mathrm{Geom}} \cdot P_{\mathrm{T}}$ is relative to the nominal dilepton branching fraction (4/81) of $t \bar{t}$ events. 125

5.7 Summary of the uncertainties in the detection efficiency $(\Delta \varepsilon / \varepsilon)$ and the

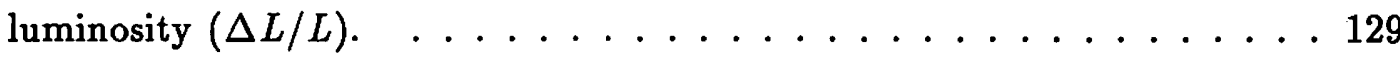


5.8 The efficiencies for various background processes. The cross section multiplied by the braching ratio for each channel $\sigma \cdot \mathrm{BR}$ shown in the table is taken from the ISAJET calculation. The $\varepsilon_{\text {Total }}$ is relative to the number of events produced times the branching ratio for the channel to be considered. The statistical errors are shown for the total detection efficiencies $\varepsilon_{\text {Total }} \ldots \ldots \ldots \ldots \ldots \ldots \ldots$

5.9 The efficiency of the event topology cut and its breakdown, for each background process. The errors are statistical. Note that only the back-toback topology cut is applied in the $e \mu$ channel. . . . . . . . . . . . 131

5.10 The expected number of background events at $4.1 \mathrm{pb}^{-1}$ before and after the event topology cut with the statistical error. . . . . . . . . . . 131

5.11 The probabilities for real leptons $\left(p_{\text {real }}\right)$, and for fake leptons $\left(p_{\text {fake }}\right)$ to pass the identification cut. Isolated EM clusters in a jet event sample are considered as fake electrons. As fake muons, all the isolated tracks in a jet event sample are considered. The statistical errors are shown in this

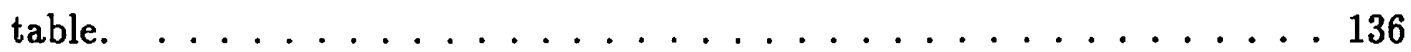

5.12 The number of fake dilepton backgrounds expected in $4.1 \mathrm{pb}^{-1}$. The errors

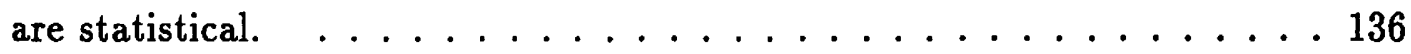

6.1 Poisson upper limits for $n_{0}$ observed events. . . . . . . . . . . 161

6.2 Upper limits for $n_{0}$ observed events at 95\% C.L. using a Poisson-Gaussian convoluted probability function with the systematic uncertainty of $\sigma / \mu$.

6.3 The upper limits on the $t \bar{t}$ production cross section from the high $P_{\mathrm{T}}$ dilepton analysis and the combined analysis including the low $P_{\mathrm{T}}$ muon

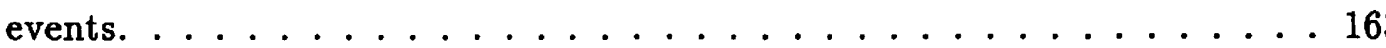




\section{List of Figures}

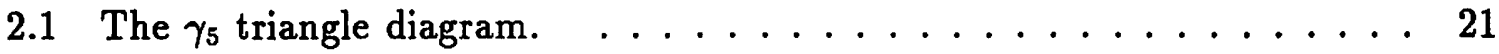

2.2 The box diagrams for (a) $B_{d}^{0}-\bar{B}_{d}^{0}$ and (b) $B_{s}^{0}-\bar{B}_{s}^{0}$ mixing. . . . . . . 22

2.3 The diagrams for the radiative correction to the weak interaction. . . . 23

2.4 The radiative correction factor to the $W$ mass, $\Delta r$, as a function of $M_{\text {top }}$

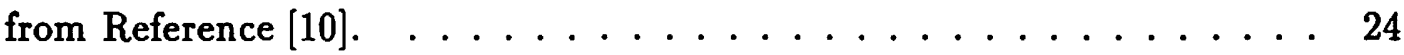

2.5 The $t \bar{t}$ production cross section in $p \bar{p}$ collisions by Altarelli et. al. [23], based on the next-to-leading order calculation by Nason, Dawson and

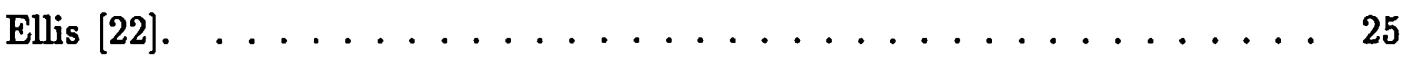

2.6 The lowest order Feynman diagrams for the heavy quark pair production. 26

2.7 The $t \bar{t}$ production cross section as a function of the scale parameter $\mu$. . $\quad 27$

2.8 The $t \bar{t}$ production cross section at $\sqrt{s}=1.8 \mathrm{TeV}$ by Ellis [24], based on the next-to-leading order calculation by Nason, Dawson and Ellis [22]. . 28

2.9 The decay width of the top quark as a function of the top quark mass. . 29

2.10 The fraction of the $t \bar{t}$ production for which the fragmentation occurs before the top quark decays, as a function of the top quark mass, for $1.8-\mathrm{TeV}$ $p \bar{p}$ collisions. Solid line: hadronization scale $\Lambda^{-1}=1 \mathrm{fm}$. Dashed line: $\Lambda^{-1}=0.5 \mathrm{fm}$. Dotted line: $\Lambda^{-1}=2 \mathrm{fm} . \ldots \ldots . \ldots 30$

3.1 A perspective view of the CDF detector showing the central detector and the forward and backward detectors. . . . . . . . . . . . . 44

3.2 An elevation view through the forward half of the CDF. The detector is forward-backward symmetric about the interaction point. . . . . . . 45 
3.3 A beam's-eye view of one of the beam-beam counter planes. . . . . . . 46

3.4 Two of the eight Vertex Time Projection Chamber modules. . . . . . . . 47

3.5 An endplate of the Central Tracking Chamber showing the arrangement of the blocks which hold the 84 layers of sense wires. . . . . . . . . 48

3.6 One of 48 wedges, showing the central EM calorimeter with the lightgathering system.

3.7 Quadrant of the calorimeter where A, B, C show central, endwall and plug, respectively. ................... 50

3.8 Cross sectional view of the end plug electromagnetic calorimeter. . . . . 51

3.9 Isometric view of a PEM quadrant, showing the projective pad tower structure and the longitudinal layers. . . . . . . . . . 52

3.10 The layout of the central muon chambers in one wedge. . . . . . . 53

3.11 The arrangement of the four planes of the central muon chambers in a view along the beam direction $\left(\Delta \phi=4.2^{\circ}\right) \ldots \ldots \ldots \ldots$

4.1 The $\chi_{\text {strip }}^{2}$ for central electrons from $Z \rightarrow e e$ and jet samples. . . . . 84

4.2 The average strip chamber $\chi^{2}=\left(\chi_{\text {strip }}^{2}+\chi_{\text {wire }}^{2}\right) / 2$ distributions for test beam electrons, test beam charged pions and $W$ electrons. . . . . . 85

4.3 The $H A D /(E M+H A D)$ ratio for test beam electrons, test beam $\pi$ 's and electrons from $W \rightarrow e \nu$ events. . . . . . . . . . . . 86

4.4 The ratio of the calorimeter energy and the momentum, $E / P$, for central electrons from $Z \rightarrow e e$ and jet samples. . . . . . . . . . 87

4.5 The position match in $R-\phi$ view $\left(D_{X}\right)$ and in $z$ direction $\left(D_{Z}\right)$ for central electrons from $Z \rightarrow e e$ and jet samples. . . . . . . . . . 88

4.6 The lateral shower profile variable $\left(L_{\text {shr }}\right)$ for central electrons from $Z \rightarrow e e$ and jet samples. . . . . . . . . . . . . . . . . 89

4.7 The radial distance of the conversion points from central conversion elec-

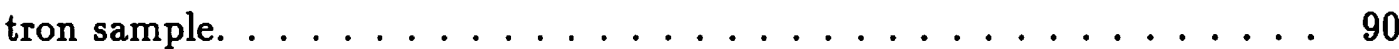


4.8 The isolation distribution for central electrons from the ISAJET+CDFSIM $t \bar{t}$ Monte Carlo with $M_{\text {top }}=90 \mathrm{GeV} / c^{2} \ldots \ldots \ldots . \ldots 1$

4.9 The predicted number of dilepton events as a function of common $E_{\mathrm{T}}\left(P_{\mathrm{T}}\right)$ threshold on both leptons from Reference [19]. . . . . . . . . . . . 92

4.10 The ratio of the hadronic energy and the electromagnetic energy for plug electrons from $Z \rightarrow e e$ and jet samples. . . . . . . . . . . 93

4.11 The lateral shape chi-squared of the EM shower, $\chi_{3 \times 3}^{2}$, for plug electrons from $Z \rightarrow e e$ and jet samples. . . . . . . . . . . . . . . 94

4.12 The VTPC hit occupancy, $f_{\mathrm{VTPC}}$, for plug electrons from $Z \rightarrow e e$ and jet

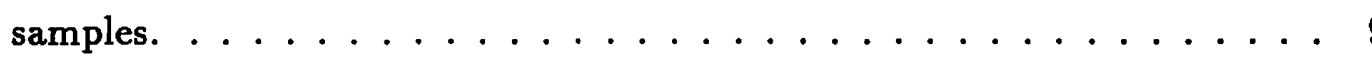

4.13 Track matching for plug electrons from $Z \rightarrow e e$ and jet samples. (a) The CTC $\phi$ matching, (b) the CTC radial distance matching, (c) the VTPC $\phi$ matching, and (d) the VTPC radial distance matching. . . . . . . . 9

4.14 The isolation distribution for plug electrons from the ISAJET+CDFSIM $t \bar{t}$ Monte Carlo with $M_{\text {top }}=90 \mathrm{GeV} / c^{2}$. . . . . . . . . . 97

4.15 The energy distribution of the tower through which a muon traverses from test beam data. (a) The electromagnetic energy $E M_{\mu}$ and (b) the hadronic energy $H A D_{\mu} \ldots \ldots \ldots \ldots \ldots$. . . . . . . . . 98

4.16 The electromagnetic tower energy $E M_{\mu}$ through which a muon traverses. The distributions from $Z \rightarrow \mu \mu$ and jet samples are shown. . . . . . . 99

4.17 The hadronic tower energy $H A D_{\mu}$ through which a muon traverses. The distributions from $Z \rightarrow \mu \mu$ and jet samples are shown. . . . . . . 100

4.18 The position matching in $R-\phi$ view, $D_{X}^{\mu}$, between the CTC track and the CMU chamber hits for central muons from $Z \rightarrow \mu \mu$ and jet samples. . . . 101

4.19 The distributions of (a) calorimeter isolation and (b) track isolation, for central muons from the ISAJET+CDFSIM Monte Carlo with $M_{\text {top }}=90$ $\mathrm{GeV} / c^{2} \ldots \ldots \ldots \ldots \ldots \ldots$ 
4.20 The opening angle $\Delta \phi$ between a muon and a jet in an inclusive muon sample.

4.21 The azimuthal opening angle $\Delta \phi_{c \mu}$ between an electron and a muon for $e \mu$ events from (a) the $t \bar{t}$ Monte Carlo with $M_{\text {top }}=90 \mathrm{GeV} / c^{2}$, (b) $b \bar{b}$ Monte Carlo, and (c) $Z \rightarrow \tau \tau$ Monte Carlo. . . . . . . . . . . . . . 104

4.22 The invariant mass $M_{\ell}$ of dielectron or dimuon pairs from (a) the $t \bar{t}$ Monte Carlo with $M_{\mathrm{top}}=90 \mathrm{GeV} / c^{2}$, (b) $b \bar{b}$ Monte Carlo, and (c) $Z, \gamma \rightarrow e e, \mu \mu$ Monte Carlo. . . . . . . . . . . . . . . . . . . . 105

4.23 Scatter plots of $\Delta \phi_{\ell \ell}$ versus $\psi_{\mathrm{T}}$ for $e e, \mu \mu$ events from (a) the $t \bar{t}$ Monte Carlo with $M_{\text {top }}=90 \mathrm{GeV} / c^{2}$, (b) $b \bar{b}$ Monte Carlo, and (c) $Z, \gamma \rightarrow e e, \mu \mu$ Monte Carlo. . . . . . . . . . . . . . . . . . 106

4.24 Scatter plots for the $4 e \mu$ events. (a) The electron $E_{\mathrm{T}}$ versus the muon $P_{\mathrm{T}}$. (b) The transverse energy or the tranverse momentum of the least energetic lepton versus the opening angle between $e$ and $\mu . \ldots 107$

4.25 The invariant mass distributions for the dielectron and dimuon CDF data (histograms) together with the $Z, \gamma \rightarrow e e, \mu \mu$ Monte Carlo (curves). . . . 108

4.26 The scatter plots of (a) the opening angle between two electrons versus $\mathbb{F}_{\mathrm{T}}$ for the CDF dielectron data and (b) the opening angle between two muons versus $\not_{\mathrm{T}}$ from the CDF dimuon data. . . . . . . . . . . 109

5.1 The distance in $\eta-\phi$ space between GENP leptons from top quark decays and the nearest reconstructed lepton for (a) electrons with $E_{\mathrm{T}}>15 \mathrm{GeV}$ and (b) muons with $P_{\mathrm{T}}>15 \mathrm{GeV} / c . M_{\mathrm{top}}=90 \mathrm{GeV} / c^{2} \ldots \ldots 138$

5.2 Efficiency for a top lepton to have a matched lepton reconstructed by the offline codes as a function of lepton $E_{\mathrm{T}} / P_{\mathrm{T}}$ for (a) electrons and (b) muons. $M_{\mathrm{top}}=90 \mathrm{GeV} / c^{2}$.

5.3 The isolation cut efficiency for electrons from $M_{\text {top }}=90 \mathrm{GeV} / c^{2}$ ISAJET Monte Carlo data as a function of $\eta$. The detector simulation is not applied. 
5.4 The isolation cut efficiency for the central electrons from $M_{\text {top }}=90$ $\mathrm{GeV} / c^{2}$ ISAJET Monte Carlo data as a function of $E_{\mathrm{T}}$. The detector simulation is not applied. . . . . . . . . . . . . . 141

5.5 Comparison of the lepton isolation distributions for the CDF $Z$ data and the ISAJET + CDFSIM $Z$ Monte Carlo. For the Monte Carlo events, the cut $E_{\mathrm{T}}\left(P_{\mathrm{T}}\right)>15 \mathrm{GeV}(\mathrm{GeV} / c)$ is imposed on leptons. For leptons in the CDF data, all the lepton selection cuts except the isolation are required. 142

5.6 Comparison of the lepton isolation distributions for the low $E_{\mathrm{T}} \mathrm{CDF}$ $e \mu$ events and the ISAJET + CDFSIM $b \bar{b}$ Monte Carlo. For the Monte Carlo events, the cut $E_{\mathrm{T}}\left(P_{\mathrm{T}}\right)>7 \mathrm{GeV}(\mathrm{GeV} / c)$ is imposed on leptons. For leptons in the CDF data, all the lepton selection cuts except the isolation requirement are required. . . . . . . . . . . . . . 143

5.7 The isolation distributions for central electrons from the $t \bar{t}$ Monte Carlo with $M_{\text {top }}=90 \mathrm{GeV} / c^{2}$, the CDF $Z \rightarrow$ ee Monte Carlo and the $b \bar{b}$ Monte Carlo. . . . . . . . . . . . . . . . . . . . 144

5.8 Comparison of the central electron identification variables for the CDF and Monte Carlo $Z \rightarrow e e$ events. . . . . . . . . . . . . 145

5.9 Comparison of the central electron identification variables for the CDF and Monte Carlo $Z \rightarrow e e$ events. . . . . . . . . . . . . . . 146

5.10 The central electron identification efficiency as a function of the isolation calculated from $M_{\mathrm{top}}=90 \mathrm{GeV} / c^{2}$ ISAJET Monte Carlo + CDFSIM

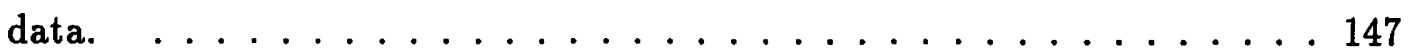

5.11 The CTC and VTPC track matching efficiency as a function of $\eta$ for the plug $Z$ and $W$ events. . . . . . . . . . . . . . . . 148

5.12 The track matching (CTC or VTPC) efficiency as a function of $\eta$ for the plug $Z$ and $W$ events. . . . . . . . . . . . . . 149

5.13 The electron identification cut efficiency and the background reduction factor of the cut for the central electron and the plug electron. . . . 150 
5.14 The trigger efficiencies as a function of electron $E_{\mathrm{T}}$ or muon $P_{\mathrm{T}}$ for the central electron, the plug electron and the central muon triggers. . . . 151

5.15 The top quark mass dependence of the cut efficiencies and the total detection efficiency. . . . . . . . . . . . . . . . . 152

5.16 The GENP isolation for the central electrons (a), and contributions from (b) the hard collision, (c) initial state radiation, and (d) beam jet. The $t \bar{t}$ Monte Carlo with $M_{\text {top }}=90 \mathrm{GeV} / c^{2}$ is used for the plots. . . . . . 153

5.17 The GENP isolation from the hard collision for the central electron (a), and the contributions from (b) the top quark decay, (c) top quark fragmentation, and (d) final state radiation. The $t \bar{t}$ Monte Carlo with $M_{\text {top }}=$ $90 \mathrm{GeV} / c^{2}$ is used for the plots. . . . . . . . . . . . . . . 154

5.18 Comparison of the $\mathbb{W}_{\mathrm{T}}$ distributions for (a) the CDF central $W \rightarrow e \nu$ data and the Monte Carlo, and (b) the CDF plug $W \rightarrow e \nu$ data and the Monte Carlo. . . . . . . . . . . . . . . . . 155

5.19 The event topology cut efficiency $\varepsilon_{\text {Event }}$ for $Z \rightarrow e e, \mu \mu$ Monte Carlo events as a function of $P_{\mathrm{T}}^{Z, \gamma} \ldots \ldots \ldots \ldots \ldots$

5.20 The transverse momentum $P_{\mathrm{T}}^{Z}$ distribution from CDF $Z \rightarrow$ ee events. $\quad 157$

5.21 The distribution of the azimuthal opening angle, $\Delta \phi_{c \mu}$, between an electron and a muon from low $E_{\mathrm{T}} e \mu$ events. (a) CDF data and ISALEP Monte Carlo prediction. (b) The contribution from $b \bar{b}$ direct production process in the Monte Carlo data. (c) The contribution from gluon splitting process in the Monte Carlo data. . . . . . . . . . . . 158

6.1 A Poisson distribution and a modified distribution (Equation 6.7) for

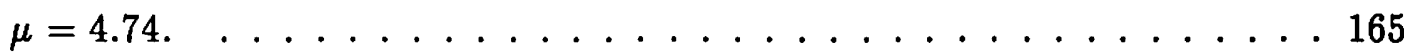


6.2 The $95 \%$ confidence level limits on $\sigma(t \bar{t})$ compared with a band of theoretical predictions from Reference [24]. We show three sets of experimental limits: (1) From the $e \mu$ analysis of Reference [19], (2) From the analysis of the dilepton modes, (3) From the combination of the dilepton analysis and the low $P_{\mathrm{T}}$ muon analysis. ................... 166 


\section{The CDF Collaboration}

F. Abe, ${ }^{8}$ D. Amidei, ${ }^{4}$ G. Apollinari, ${ }^{11}$ M. Atac,${ }^{4}$ P. Auchincloss, ${ }^{14}$ A. R. Baden, ${ }^{6}$

A. Bamberger, ${ }^{a}$ A. Barbaro-Galtieri, ${ }^{9}$ V. E. Barnes, ${ }^{12}$ F. Bedeschi, ${ }^{11}$ S. Behrends, ${ }^{2}$ S. Belforte, ${ }^{11}$ G. Bellettini, ${ }^{11}$ J. Bellinger,${ }^{18} \mathrm{~J}$. Bensinger, ${ }^{2}$ A. Beretvas, ${ }^{4}$ J. P. Berge,${ }^{4}$

S. Bertolucci, ${ }^{5}$ S. Bhadra,${ }^{7}$ M. Binkley, ${ }^{4}$ R. Blair, ${ }^{1}$ C. Blocker,${ }^{2}$ A. W. Booth,${ }^{4}$ G. Brandenburg, ${ }^{6}$ D. Brown, ${ }^{6}$ E. Buckley, ${ }^{14}$ A. Byon, ${ }^{12}$ K. L. Byrum, ${ }^{18}$ C. Campagnari, ${ }^{3}$ M. Campbell, ${ }^{3}$ R. Carey, ${ }^{6}$ W. Carithers, ${ }^{9}$ D. Carlsmith, ${ }^{18}$ J. T. Carroll, ${ }^{4}$ R. Cashmore, ${ }^{a}$ F. Cervelli, ${ }^{11}$ K. Chadwick, ${ }^{4}$ G. Chiarelli, ${ }^{5}$ W. Chinowsky, ${ }^{9}$ S. Cihangir ${ }^{4}$ A. G. Clark, ${ }^{4}$ D. Connor, ${ }^{10}$ M. Contreras, ${ }^{2}$ J. Cooper,${ }^{4}$ M. Cordelli, ${ }^{5}$ D. Crane, ${ }^{4}$ M. Curatolo, ${ }^{5}$ C. Day, ${ }^{4}$ S. Dell'Agnello, ${ }^{11}$ M. Dell'Orso, ${ }^{11}$ L. Demortier, ${ }^{2}$ P. F. Derwent, ${ }^{3}$ T. Devlin, ${ }^{14}$ D. DiBitonto, ${ }^{15}$ R. B. Drucker, ${ }^{9}$ J. E. Elias, ${ }^{4}$ R. Ely, ${ }^{9}$ S. Errede, ${ }^{7}$ B. Esposito, ${ }^{5}$ B. Flaugher, ${ }^{14}$ G. W. Foster, ${ }^{4}$ M. Franklin, ${ }^{6}$ J. Freeman,${ }^{4}$ H. Frisch,${ }^{3}$ Y. Fukui, ${ }^{8}$ Y. Funayama, ${ }^{16}$ A. F. Garfinkel, ${ }^{12}$ A. Gauthier,${ }^{7}$ S. Geer,${ }^{6}$ P. Giannetti, ${ }^{11}$ N. Giokaris,${ }^{13}$ P. Giromini, ${ }^{5}$ L. Gladney, ${ }^{10} \mathrm{M}$. Gold, ${ }^{9} \mathrm{~K}$. Goulianos, ${ }^{13} \mathrm{H}$. Grassmann, ${ }^{11} \mathrm{C}$. Grosso-Pilcher, ${ }^{3}$ C. Haber, ${ }^{9}$ S. R. Hahn, ${ }^{4}$ R. Handler, ${ }^{18}$ K. Hara,${ }^{16}$ R. M. Harris,${ }^{9}$ J. Hauser,${ }^{3}$ T. Hessing, ${ }^{15}$

R. Hollebeek, ${ }^{10}$ L. Holloway, ${ }^{7}$ P. Hu, ${ }^{1 / 1}$ B. Hubbard, ${ }^{9}$ B. T. Huffman, ${ }^{12}$ R. Hughes, ${ }^{10}$ P. Hurst,${ }^{7}$ J. Huth,${ }^{4}$ M. Incagli, ${ }^{11}$ T. Ino, ${ }^{16}$ H. Iso,${ }^{16}$ H. Jensen, ${ }^{4}$ C. P. Jessop, ${ }^{6}$ R. P. Johnson, ${ }^{4}$ U. Joshi, ${ }^{4}$ R. W. Kadel,${ }^{4}$ T. Kamon, ${ }^{15}$ S. Kanda, ${ }^{16}$ D. A. Kardelis, ${ }^{7}$ I. Karliner, ${ }^{7}$ E. Kearns, ${ }^{6}$ R. Kephart, ${ }^{4}$ P. Kesten, ${ }^{2}$ R. M. Keup,${ }^{7}$ H. Keutelian, ${ }^{7}$ S. Kim, ${ }^{16}$ L. Kirsch, ${ }^{2}$ K. Kondo, ${ }^{16}$ S. E. Kuhlmann, ${ }^{1}$ E. Kuns, ${ }^{14}$ A. T. Laasanen, ${ }^{12}$ J. I. Lamoureux, ${ }^{18}$ W. Li, ${ }^{1}$ T. M. Liss, ${ }^{7}$ N. Lockyer, ${ }^{10}$ C. B. Luchini, ${ }^{7}$ P. Maas, ${ }^{4}$ M. Mangano ${ }^{11}$ J. P. Marriner,${ }^{4}$ R. Markeloff, ${ }^{18}$ L. A. Markosky, ${ }^{18}$ R. Mattingly, ${ }^{2}$ P. McIntyre,,${ }^{15}$ A. Menzione, ${ }^{11}$ T. Meyer, ${ }^{15}$ S. Mikamo, ${ }^{8}$ M. Miller, ${ }^{3}$ T. Mimashi, ${ }^{16}$ S. Miscetti, ${ }^{5}$ M. Mishina, ${ }^{8}$ S. Miyashita, ${ }^{16}$ Y. Morita, ${ }^{16}$ S. Moulding, ${ }^{2}$ A. Mukherjee,${ }^{4}$ L. F. Nakae, ${ }^{2}$ I. Nakano, ${ }^{16}$ C. Nelson, ${ }^{4}$ C. Newman-Holmes, ${ }^{4}$ J. S. T. Ng, ${ }^{6}$ M. Ninomiya, ${ }^{16}$ L. Nodulman, ${ }^{1}$ S. Ogawa, ${ }^{16}$ R. Paoletti, ${ }^{11}$ A. Para, ${ }^{4}$ E. Pare, ${ }^{6}$ J. Patrick, ${ }^{4}$ T. J. Phillips, ${ }^{6}$ R. Plunkett, ${ }^{4}$ L. Pondrom,${ }^{18}$ J. Proudfoot,${ }^{1}$ G. Punzi, ${ }^{11}$ D. Quarrie, ${ }^{4}$ K. Ragan, ${ }^{10}$ G. Redlinger,${ }^{3}$ J. Rhoades, ${ }^{18}$ M. Roach, ${ }^{17}$ F. Rimondi, ${ }^{a}$ L. Ristori, ${ }^{11}$ T. Rohaly, ${ }^{10}$ A. Roodman, ${ }^{3}$ A. Sansoni, ${ }^{5}$ R. D. Sard, ${ }^{7}$ A. Savoy-Navarro, ${ }^{a}$ V. Scarpine, ${ }^{7}$ P. Schlabach, ${ }^{7}$ E. E. Schmidt, ${ }^{4}$ M. H. Schub,${ }^{12}$ R. Schwitters,${ }^{6}$ A. Scribano, ${ }^{11}$ S. Segler, ${ }^{4}$ Y. Seiya, ${ }^{16}$ M. Sekiguchi, ${ }^{16}$ P. Sestini, ${ }^{11}$ M. Shapiro, ${ }^{6}$ M. Sheaff, ${ }^{18}$ M. Shochet,${ }^{3}$ J. Siegrist, ${ }^{9}$ P. Sinervo, ${ }^{10}$ J. Skarha, ${ }^{18}$ K. Sliwa, ${ }^{17}$ D. A. Smith, ${ }^{11}$ F. D. Snider, ${ }^{3}$ R. St. Denis,${ }^{6}$

A. Stefanini, ${ }^{11}$ R. L. Swartz, Jr. ${ }^{7}$ M. Takano, ${ }^{16}$ K. Takikawa, ${ }^{16}$ S. Tarem, ${ }^{2}$ D. Theriot,${ }^{4}$

M. Timko, ${ }^{15}$ P. Tipton,${ }^{9}$ S. Tkaczyk, ${ }^{4}$ A. Tollestrup ${ }^{4}$ G. Tonelli, ${ }^{11} \mathrm{~J}$. Tonnison, ${ }^{12}$ W. Trischuk, ${ }^{6}$ Y. Tsay, ${ }^{3}$ F. Ukegawa, ${ }^{16}$ D. Underwood, ${ }^{1}$ R. Vidal,${ }^{4}$ R. G. Wagner, ${ }^{1}$ R. L. Wagner, ${ }^{4}$ J. Walsh, ${ }^{10}$ T. Watts, ${ }^{1.4}$ R. Webb,${ }^{15}$ C. Wendt,${ }^{18}$ W. C. Wester, $\Pi 1,{ }^{9}$ T. Westhusing, ${ }^{11}$ S. N. White, ${ }^{13}$ A. B. Wicklund, ${ }^{1}$ H. H. Williams, ${ }^{10}$ B. L. Winer,${ }^{9}$ A. Yagil, ${ }^{4}$ A. Yamashita, ${ }^{16}$ K. Yasuoka, ${ }^{16}$ G. P. Yeh, ${ }^{4}$ J. Yoh, ${ }^{4}$ M. Yokoyama,${ }^{16}$ J. C. Yun, ${ }^{4}$ F. Zetti ${ }^{11}$

\footnotetext{
1 Argonne National Laboratory, Argonne, Illinois 60439

2 Brandeis University, Waltham, Massachusetts 02254

3 University of Chicago, Chicago, Mlinois 60637

4 Fermi National Accelerator Laboratory, Batavia, Illinois 60510

5 Laboratori Nazionali di Frascati, Istituto Nazionale di Fisica Nucleare, Frascati, Italy

6 Harvard University, Cambridge, Massachusetts 02138
} 
7 University of Mlinois, Urbana, Illinois 61801

8 National Laboratory for High Energy Physics (KEK), Tsukuba, Ibaraki 305, Japan

9 Lawrence Berkeley Laboratory, Berkeley, California 94720

10 University of Pennsylvania, Philadelphia, Pennsylvania 19104

11 Istituto Nazionale di Fisica Nucleare, University and Scuola Normale Superiore of Pisa, I-56100 Pisa, Italy

12 Purdue University, West Lafayette, Indiana 47907

13 Rockefeller University, New York, New York 10021

14 Rutgers University, Piscataway, New Jersey 08854

15 Texas A\&M University, College Station, Texas 77843

16 University of Tsukuba, Tsukuba, Ibaraki 305, Japan

17 Tufts University, Medford, Massachusetts 02155

18 University of Wisconsin, Madison, Wisconsin 53706

a Visitor 


\section{Chapter 1}

\section{Introduction}

Since a unified theory of the electromagnetic interaction and the weak interaction was first proposed in 1960's [1], a gauge theory based on $S U(3)_{\mathrm{QCD}} \times S U(2)_{\mathrm{L}} \times U(1)$ group, now called the Standard Model, has been making a great success in describing interactions of elementary particles. In this model, only the top quark and the Higgs boson are yet to be observed. This thesis describes a search for the top quark in $p \bar{p}$ collisions at $\sqrt{s}=1.8 \mathrm{TeV}$. The data with an integrated luminosity of $4.1 \mathrm{pb}^{-1}$ were collected by the CDF detector during the 1988-1989 Tevatron run.

In the remainder of Chapter 1 , we briefly review a historical development of the particle physics with an emphasis on the phenomenology, and try to clarify where we stand now in particle physics. Chapter 2 describes our understanding of the top quark in the framework of the Standard Model. Chapter 3 describes the CDF detector relevant to this analysis where a search for the top quark in dilepton events $(e \mu, e e$ and $\mu \mu)$ is performed. In Chapter 4, our event selection criteria for the top quark search is described. Only one event remained, passing all the selection cuts. From this result, we derive a lower mass limit on the top quark in succeeding chapters. Chapter 5 describes the determination of the detection efficiency for the top events which is necessary for deriving a mass limit. A mass limit calculation is performed in Chapter 6, and Chapter 7 concludes this thesis. 
Today, most of physicists believe in the top quark. It is quite contrary to the situation in 1890's when most of physicists doubted even the existence of the electron. Before 1900, there was no definitive picture about the structure of the atom. It was uncovered when J. J. Thomson first observed electrons and Rutherford with his co-workers obtained an evidence for the nucleus early in 1900's. When the periodic law according to the atomic number was developed by Moseley in 1914, we reached an understanding of the atom structure.

The origin of the great development of the elementary particle physics can be traced back to elaborate studies on the nucleus, only where one could observe strong interactions and weak interactions. One cannot miss the construction of the Quantum Mechanics in 1920's, which has turned out to be indispensable for describing particle interactions in the nucleus. The structure of the nucleus was made clear when Rutherford proposed the neutron as a constituent of nuclei together with the proton in 1920, which was experimentally confirmed by Chadwick in 1932 . The neutron had a distinctive feature that it was almost a carbon copy of the proton except the electric charge it carries. This fact inspired the concept of the isospin. This concept due to Heisenberg is an origin of today's prescription of treating particles in a multiplet. Another great theoretical achievement in strong interactions was a prediction of the pion by Yukawa in 1935. The pion was thought to mediate the strong force between nucleons. At almost the same time (1934), Fermi proposed a theory for the $\beta$ decay by introducing the neutron and the neutrino $\nu$ which was first suggested by Pauli in 1930. The pion and the neutrino were experimentally observed later.

By the end of 1940's, we had already known the existence of the proton, the neutron, the photon, the electron (and the positron), the pion and the muon. It seemed that we had obtained a complete list of the elementary particles. The successive discoveries of vast varieties of strange particles, however, changed such a thought. The first observation of these unexpected particles in 1947 was for kaons, called "V-particles" at that time, in the secondary particles of cosmic rays. To account for a strange characteristics of 
the strange particles that they were produced with a large production cross section while they had a long life time, a new quantum number "strangeness" was introduced. Physicists also found that the strange particles together with the known hadrons were arranged in a certain manner. The situation was similar to the case when the atoms were arranged according to the atomic number. The arrangement is, however, much more complicated this time and required a mathematical treatment based on the group theory. Physicists constructed various models to explain the variety of particles from several basic or "elementary" particles. At first, some of the baryons were considered as such elementary particles. The Sakata model assumed that all the particles were composed of the combination of $p, n, \Lambda$ and their antiparticles. This idea based on $S U(3)$ group made a great success in describing the meson multiplet, leading to a prediction of the $\eta$ particle. But it could not reproduce the observed baryon multiplet.

The $S U(3)$-based eight-multiplet ("eightfold way") by Gell-Mann and Ne'eman succeeded in describing the hadron multiplet. It gave rise to a prediction of the $\Omega$ particle, which was experimentally observed in 1964. Thus Physicists sought for the "elementary particles" which were consistent with the $S U(3)$ and well described the observed arrangement of hadrons. In 1964, Gell-Mann and Zweig proposed a new $S U(3)$ model with three elementary particles called "quark"s. Three quarks, named up quark $(u)$, down quark $(d)$ and strange quark $(s)$, carried fractional charges and fractional baryon numbers. In this model, all the baryons were thought to consist of three quarks while the mesons were thought to consist of a quark and an antiquark.

The quark model is a main part of the current picture for the elementary particle physics. Based on the quark model, the Cabbibo theory suceeded to describe weak interactions. As for strong interactions, the Quantum Chromodynamics (QCD) was developed where the color was an equivalent of the charge in electromagnetic interactions and the strong force was mediated by gluons between quarks.

Just as physicists wanted to describe observed particles from the elementary particles, they were tempted to unify the known four interactions into one "elementary 
interaction". The first unification of the electromagnetic and the weak interaction into an electroweak interaction was performed by Glashow, Weinberg and Salam in 1960's. In this model, now called the Standard Model together with the QCD, the weak force was mediated by heavy bosons, $W^{ \pm}$and $Z^{0}$. The masses of the elementary particles were generated via interactions with a new particle, the Higgs boson. The first observation in early 1980's of the $W$ and $Z$ in $p \bar{p}$ collisions at masses predicted by the Standard Model was considered as a great success of this theory.

The first heavy quark, the charm quark, was observed in 1972 as a quarkonium state $(J / \psi)$. The existence of the charm quark had been predicted by the Standard Model in order to give a systematic description of electroweak interactions being consistent with the experimental results. According to the Standard Model, the quarks and leptons form a pair called a weak isospin doublet. The $u$ and the $d$ quark form a doublet, and the $s$ quark needs its partner which is the charm quark. Such an arrangement shows an apparent correspondence between quarks and leptons where the electron (muon) forms a weak isospin doublet together with the electron(muon)-neutrino. When the $\tau$ lepton was discovered in 1975, the existence of its counterpart in the quark sector was envisaged and it was actually observed in 1977 as a quarkonium state $\Upsilon$. It was found to have a same charge as the $d$ and the $s$ quarks. 


\section{Chapter 2}

\section{Top Quark in the Standard Model}

One can see that we now stand in almost the same situation as that just before the discovery of the charm quark. We believe in the top quark for essentially the same reason that was argued for the charm quark twenty years ago. The following subsections describe in more detail why we believe the existence of the top quark and the current understandings on the top quark in the Standard Model.

\subsection{Indication of the Existence of the Top Quark}

In the framework of the Standard Model $[1,2,3]$, there are several indications of the existence of the top quark.

\subsubsection{Phenomenological Evidences}

Forward-backward Asymmetry in $e^{+} e^{-} \rightarrow b \bar{b}$

The existence of the top quark is suggested indirectly by observing that the $b$ quark belongs to a weak isospin doublet in the Standard Model. The forward-backward asymmetry of the $b$ quark production in the $e^{+} e^{-} \rightarrow b \bar{b}$ process provides one of the experimental chances which determine whether the $b$ quark forms a doublet or is a singlet in the Standard Model. 
The angular distribution of the $b$ quark in the process $e^{+} e^{-} \rightarrow b \bar{b}$ is given in the form

$$
\frac{d \sigma}{d(\cos \theta)}=A\left(1+\cos \theta^{2}\right)+B \cos \theta
$$

As a consequence of the neutral weak interaction, an asymmetry term, the second term of the right hand side in Equation (2.1), appears. The coefficients, $A$ and $B$, are functions of the eigenvalues of the operators $T_{3}$ and $Q$ for the $b$ quark, where $T_{3}$ is the third component of the weak isospin and $Q$ is the charge operator. If the left-handed $b$ quark $\left(b_{\mathrm{L}}\right)$ is a member of the weak isospin doublet, the corresponding eigenvalue for $T_{3}$ is $T_{3 \mathrm{~L}}^{b}=-1 / 2$, while $T_{3 \mathrm{~L}}^{b}=0$ for a singlet $b_{\mathrm{L}}$. Integrating Equation (2.1) in the forward $(0 \leq \cos \theta \leq 1)$ and the backward $(-1 \leq \cos \theta \leq 0)$ region, we can define a parameter describing the forward-backward asymmetry as a function of $T_{3 \mathrm{~L}}$ :

$$
\begin{aligned}
A_{\mathrm{FB}} & \equiv \frac{N_{b}^{\mathrm{F}}-N_{b}^{\mathrm{B}}}{N_{b}^{\mathrm{F}}+N_{b}^{\mathrm{B}}} \\
& \propto T_{3 \mathrm{~L}}^{b}\left(T_{3 \mathrm{~L}}^{b}+\frac{2}{3} \sin ^{2} \theta_{\mathrm{W}}\right)
\end{aligned}
$$

The recent measurement of the forward-backward asymmetry of the $b$ quark in the $e^{+} e^{-} \rightarrow b \bar{b}$ process was performed by the JADE collaboration [4], giving a result of

$$
a_{b}=2 T_{3 \mathrm{~L}}^{b}=-0.9 \pm 0.24 \pm 0.10
$$

where muon pairs from the $b$ quark decays were used to measure the asymmetry. It indicates that the left-handed $b$ quark belongs to a weak isospin doublet, and hence that the top quark exists.

\section{Absence of the Flavour Changing Neutral Current}

According to a theoretical calculation [5], if the left-handed $b$ quark is a weak isospin singlet, we would observe the decay of the $B$ mesons via the flavor changing neutral 
current (FCNC) at a rate such that

$$
\frac{\Gamma\left(B \rightarrow \ell^{+} \ell^{-} X\right)}{\Gamma\left(B \rightarrow \ell^{+} \nu X\right)} \geq 0.12
$$

An experimental test of this prediction was performed by the CLEO collaboration [6]. They searched for the decay $B \rightarrow \ell^{+} \ell^{-} X$ in a sample of $\Upsilon(4 S)$ 's and obtained an upper limit of

$$
B r\left(B \rightarrow \ell^{+} \ell^{-} X\right)<0.0012
$$

at the $95 \%$ confidence level (C.L.).

If the left-handed $b$ quark forms a weak isospin doublet, the suppression of the flavor changing neutral current can be explained by the GIM mechanism [2] in the Standard Model. In this case, a unitary matrix, the Kobayashi-Maskawa (KM) matrix, is introduced [3]. The KM matrix is an extension of the Cabbibo matrix [7] and connects weak eigenstates $\left(d^{\prime}, s^{\prime}, b^{\prime}\right)$ and mass eigenstates $(d, s, b)$ of the down type quarks:

$$
\left(\begin{array}{c}
d^{\prime} \\
s^{\prime} \\
b^{\prime}
\end{array}\right)=\left(\begin{array}{lll}
V_{u d} & V_{u s} & V_{u b} \\
V_{c d} & V_{c s} & V_{c b} \\
V_{t d} & V_{t s} & V_{t b}
\end{array}\right)\left(\begin{array}{c}
d \\
s \\
b
\end{array}\right) .
$$

The interaction of the neutral current and the $Z$ boson is given in terms of the Lagrangian as

$$
\begin{gathered}
\mathcal{L}=-i \frac{g}{\cos \theta_{W}}\left(j_{3}-\sin ^{2} \theta W j_{\mathrm{EM}}\right) Z \\
j_{3}-\sin ^{2} \theta_{W} j_{\mathrm{EM}}=\sum_{\text {fermion }} \bar{f}\left\{\gamma_{\mu} \frac{1}{2}\left(1-\gamma_{5}\right) T_{3}^{f}-\sin ^{2} \theta_{W} \gamma_{\mu} Q^{f}\right\} f .
\end{gathered}
$$

Since the KM matrix is a unitary matrix, the current sum over the $d^{\prime}, s^{\prime}$ and $b^{\prime}$ quarks 
is written as

$$
\begin{aligned}
& \sum_{\mathrm{d}^{\prime}, \mathbf{s}^{\prime}, \mathrm{b}^{\prime}} \bar{f}^{\prime}\left\{\gamma_{\mu} \frac{1}{2}\left(1-\gamma_{5}\right) T_{3}^{f}-\sin ^{2} \theta_{\mathrm{W}} \gamma_{\mu} Q^{f}\right\} f^{\prime} \\
= & \sum \bar{f}^{\prime} A f^{\prime} \\
= & \bar{F} V^{-1} A V F \\
= & \sum_{d, s, b} \bar{f} A f
\end{aligned}
$$

where

$$
\begin{aligned}
& A=\gamma_{\mu} \frac{1}{2}\left(1-\gamma_{5}\right) T_{3}^{f}-\sin ^{2} \theta_{W} \gamma_{\mu} Q^{f} \\
& F=\left(\begin{array}{l}
d \\
s \\
b
\end{array}\right)
\end{aligned}
$$

and $V$ is the KM matrix. The cancellation of the flavor changing neutral current as is seen in Equation (2.10) is called the GIM mechanism, which forbids the transitions such as $b \rightarrow s$.

\subsubsection{Theoretical Indication}

The renormalizability of the field theory requires a cancellation of the $\gamma_{5}$ triangle diagrams (Figure 2.1). Since each contribution of the diagrams is proportional to $T_{3}^{f}\left(Q^{f}\right)^{2}$, the sum over all the fermions leads to

$$
\begin{aligned}
& N_{\text {c.l. }}\left(-\frac{1}{2}\right)(-1)^{2}+3 N_{U}\left(\frac{1}{2}\right)\left(+\frac{2}{3}\right)^{2}+3 N_{D}\left(-\frac{1}{2}\right)\left(-\frac{1}{3}\right)^{2} \\
& =\frac{1}{2}\left(-N_{\text {c.l. }}+\frac{4}{3} N_{U}-\frac{1}{3} N_{D}\right)
\end{aligned}
$$

where $N_{\text {c.l. }}, N_{U}$ and $N_{D}$ are the number of charged leptons, up type left-handed quarks and down type left-handed quarks, respectively. The equal number of the fermion doublets for both the lepton sector and the quark sector (and therefore the existence of the top quark) realizes the cancellation of the $\gamma_{5}$ triangle diagrams. 


\subsection{Constraints on the Top Quark Mass}

The Standard Model relates the mass of the top quark to the various observables. Looking at these internal relationships and the experimental data on the relevant observables, one can derive constraints on the top quark mass. Direct searches for the top quark have also been performed in various $e^{+} e^{-}$and $p \bar{p}$ experiments. The current understanding of the top quark mass is briefly reviewed in this section.

\subsubsection{Phenomenological Constraints}

$B^{0}-\bar{B}^{0}$ Mixing

The top quark takes part in the $B^{0}-\bar{B}^{0}$ mixing via the box diagram exchanging the $W$ boson and the $u, c$ and $t$ quarks (Figure 2.2). Comparing observed level of the mixing and theoretical prediction as a function of the top quark mass, one can derive a constraint on the top quark mass. Because there are some uncertain factors such as the KM matrix elements $V_{t b}$ and $V_{t d}$, and because the structure of the $B^{0}$ meson is not known well, the lower limit on the top quark mass spreads over a somewhat wide range [8]:

$$
M_{\text {top }}>30-50 \mathrm{GeV} / c^{2}
$$

The ratio $\sigma(W \rightarrow e \nu) / \sigma(Z \rightarrow e e)$

The ratio of the cross section for the $W$ boson production followed by the decay into $e \nu$ and that for the $Z$ boson production followed by the decay into ee can be written as

$$
R=\frac{\sigma(p \bar{p} \rightarrow W X)}{\sigma(p \bar{p} \rightarrow Z X)} \cdot \frac{\Gamma(W \rightarrow e \nu)}{\Gamma(Z \rightarrow e e)} \cdot \frac{\Gamma(Z)}{\Gamma(W)}
$$

where $\Gamma(W \rightarrow e \nu)$ is the partial decay width of the $W$ for the decay mode of $W \rightarrow e \nu$, $\Gamma(W)$ is the total decay width of the $W$, and $\Gamma(Z \rightarrow e e), \Gamma(Z)$ are defined similarly. 
The top quark mass dependence of the ratio $R$ comes from the total decay width of the $W$ and $Z$ bosons. The ratio $R$ as measured by CDF [9] is

$$
R=10.2 \pm 0.8(\text { stat }) \pm 0.4(\text { sys })
$$

Using the SLAC and the LEP data for the $Z$ width [11] and a theoretical calculation for the ratio $R$ [12], CDF obtained

$$
\frac{\Gamma(W)}{\Gamma(W \rightarrow e \nu)}=9.47 \pm 0.86
$$

from which a $95 \%$ confidence level lower limit on the top quark mass was set as follows [9]:

$$
M_{\text {Lop }}>43 \mathrm{GeV} / c^{2}
$$

This result is independent of any assumptions on the decay modes of the top quark.

\section{Radiative Corrections}

The mass of the top quark and that of the Higgs boson affect the electroweak radiative corrections via virtual loops (Figure 2.3). The correction to the $W$ mass, $\Delta r$, is dependent on the top quark mass, where $\Delta r$ is defined as

$$
M_{\mathrm{I}}^{2}=\frac{\pi \alpha}{\sqrt{2} G_{\mu}} \frac{1}{(1-\Delta r) \sin ^{2} \theta_{\mathrm{W}}}
$$

Here $\alpha$ is the fine structure constant and $G_{\mu}$ is the weak coupling constant from the muon decay. As shown in Figure 2.4, $\Delta r$ is not so sensitive to the Higgs mass while it has a stronger dependence on the top quark mass. According to the comprehensive analysis given in Reference [13], an approximate upper limit on the top quark mass is set as

$$
M_{\text {top }}<200 \mathrm{GeV} / c^{2}
$$




\subsubsection{Direct Searches for the Top Quark}

Direct searches for the top quark have been performed in various collider experiments including both $e^{+} e^{-}$and $p \bar{p}$ colliders. All the detection techniques for the top quark exploit distinctive features of the top quark events due to the heavy top quark mass. As it will be discussed in $\S 2.5$, the signatures of the top quark event are the existence of isolated high $P_{\mathrm{T}}$ particles such as electrons, muons, neutrinos (missing energy), hadrons and high $E_{\mathrm{T}}$ jets.

\section{Searches in $e^{+} e^{-}$Collider Experiments}

In $e^{+} e^{-}$collisions, the top quark is produced in a pair production:

$$
e^{+} e^{-} \rightarrow t \bar{t}
$$

The top quark then decays pure-hadronically or semileptonically. The hadronic events are well identified in $e^{+} e^{-}$collisions. Since these hadrons are separated from each other (see §2.5), they distribute over a large solid angle, resulting in a spherical shape of the event. Several parameters which measure the topological shape of an event are often used in searches for the top quark in $e^{+} e^{-}$collider experiments. The commonly used parameters are the thrust, acoplanarity, sphericity and aplanarity [14]. The thrust $T$ and acoplanarity $A_{\mathrm{c}}$ are defined as

$$
\begin{aligned}
T & =\max _{\dot{\vec{n}}}\left(\frac{\sum_{i}\left|\vec{p}_{i} \cdot \hat{\vec{n}}\right|}{\sum_{i}\left|\vec{p}_{i}\right|}\right), \\
A_{c:} & =\min _{\dot{\vec{n}}}\left(4 \cdot \frac{\sum_{i}\left|\overrightarrow{p_{i}} \cdot \hat{\vec{n}}\right|}{\sum_{i}\left|\vec{p}_{i}\right|}\right)^{2},
\end{aligned}
$$

where $\vec{p}_{i}$ is the momentum of a charged or neutral particle $i$ in the center of mass frame. The thrust $T$ varies from 0.5 (isotropic distribution) to 1.0 (collinear two jets), and $A_{c}$ varies from 0 (planar distribution) to 1 (isotropic distribution). The other 2 variables 
are obtained from the momentum tensor:

$$
M_{\alpha \beta}=\sum_{i} p_{i}^{\alpha} p_{i}^{\beta}
$$

The eigenvalues of the tensor $Q_{\alpha}\left(0 \leq Q_{1} \leq Q_{2} \leq Q_{3}\right)$ are normalized so as to satisfy $Q_{1}+Q_{2}+Q_{3}=1$. The eigenvalues $Q_{1}\left(Q_{2}\right)$ and $Q_{3}$ represent the extent to which an event spreads in the $x-y$ plane (transverse to the beam direction) and along the $z$ direction (beam direction), respectively. The sphericity $S$ and the aplanarity $A$ are defined as

$$
\begin{aligned}
S & =\frac{3}{2}\left(Q_{1}+Q_{2}\right) \\
A & =\frac{3}{2} Q_{1} .
\end{aligned}
$$

The sphericity $S$ takes a value 1 for the isotropic distribution and 0 for the collinear two jets configulation. The aplanarity varies from 0 (planar distribution) to 1 (isotropic distribution).

The recent results of the top quark search in $e^{+} e^{-}$collisions are found in References $[15,16]$. the TOPAZ collaboration looked for multihadron events with an isolated muon resulting in the limit

$$
M_{\text {Lop) }}>29.9 \mathrm{GeV} / c^{2}
$$

at $95 \%$ confidence level (C.L.). By searching for spherical events containing an isolated charged particle, the ALEPH collaboration gave the following result [16]:

$$
M_{\text {top }} \notin[26.0,45.8] \mathrm{GeV} / c^{2}
$$

The ALEPH also reported a lower limit of the top quark mass of

$$
M_{\text {tup }}>45.8 \mathrm{GeV} / c^{2}
$$

at the $95 \%$ C.L. by measuring the total hadronic cross section at the $Z$ peak [16]. 


\section{Searches in $p \tilde{p}$ Collider Experiments}

In $p \bar{p}$ collisions, the top quarks are produced in pairs through strong interactions:

$$
p \vec{p} \rightarrow t \bar{t} X,
$$

and through the weak decay of the $W$ bosons if it is kinematically allowed:

$$
p \bar{p} \rightarrow W X \rightarrow t b X .
$$

Generally the searches for the top quark in $p \bar{p}$ collisions exploit the signatures distinctive for the $t \bar{t}$ events, that is, high $P_{\mathrm{T}}$ leptons and neutrinos (missing energy) with significant jet activities.

The UA1 collaboration obtained a lower mass limit on the top quark as

$$
M_{\mathrm{top}}>60 \mathrm{GeV} / c^{2}
$$

at $95 \%$ C.L. from events containing an isolated lepton and jets [17]. The UA2 collaboration has reported a lower mass limit on the top quark based on the analysis of events containing a high $P_{\mathrm{\Gamma}}$ electron and neutrino (missing $E_{\mathrm{\Gamma}}$ ) [18]. Assuming the charged current decay of the top quark, they obtained

$$
M_{\text {Lop }} \notin[30,69] \mathrm{GeV} / c^{2}
$$

at the $95 \%$ C.L..

The CDF collaboration has already obtained several limits assuming the minimal Standard Model. At the Tevatron energy, the $t \bar{t}$ production via strong interactions (2.29) dominates over the weak production (2.30) except for the top quark mass range around $60 \mathrm{GeV} / \mathrm{c}^{2}$, as shown in Figure 2.5. By searching for events containing a high $P_{\mathrm{T}}$ electron and a muon in the process (2.29), the CDF excluded the following mass rage at 
the $95 \%$ C.L. [19]:

$$
28<M_{\text {top }}<72 \mathrm{GeV} / c^{2}
$$

The decay mode where one of the top quark decays semileptonically and the other decays pure-hadronically (lepton + jets) was also studied [20]. The most important background to the high $P_{\text {r }}$ lepton plus jets events is the $W$ production followed by the leptonic decay in association with jets $(W+j e t s)$. If the top quark mass is lighter than $M_{W}+M_{b}$, the $W$ in the top quark decay is virtual, while the $W$ in the $W+$ jets process is real. In this case, the transverse mass formed by the electron or muon $P_{\mathrm{T}}$, and the missing $E_{\mathrm{T}}\left(E_{\mathrm{T}}\right)$ is smaller for the top event than that for the $W+j e t s$ event. Here the transverse mass is defined as

$$
M_{\mathrm{T}}=\sqrt{2 E_{\mathrm{T}} E_{\mathrm{T}}\left(1-\cos \phi_{e \nu}\right)}
$$

Using the different transverse mass distribution for the top events and the $W+$ jets events, the CDF obtained a lower limit of

$$
M_{\text {Lop }}>77 \mathrm{GeV} / c^{2}
$$

at the $95 \%$ C.L.. When the top quark is heavier than $M_{W}+M_{b}$, a real $W$ is produced in the top quark decay. In such a case, it is not possible to distinguish $t \bar{t}$ events from $W+j e t s$ events by the $M \cdot r$ distribution.

\subsection{Hadronic Production of the Heavy Quark}

So far, we reviewed the indications for the existence of the top quark and the searches for the top quark carried out in various collider experiments. In this section, we discuss the heavy flavor production in $p \bar{p}$ collisions.

The current theoretical strategy for treating $p \bar{p}$ interactions can be summarized as follows [21]: 
- A proton (antiproton) consists of three quarks (antiquarks), gluons and other virtual, or sea, quarks and/or antiquarks. These constituents are called partons.

- A parton $i$ in a proton has a fraction $x$ of the proton momentum with the probability described by the structure function, $f_{i}^{p}(x, \mu)$, which has to be determined experimentally. The parameter $\mu$ represents the typical energy scale in a collision.

- A $p \bar{p}$ collision is described in terms of a hard collision of one of the partons in the proton and another parton in the antiproton. The parton cross section is calculated by the Electroweak theory and the Quantum Chromodynamics (QCD).

- Other partons which did not participate in the hard collision are treated free from the collision process.

This picture of the hadron collision is expressed in the following equation:

$$
\sigma(p \bar{p} \rightarrow a b)=\sum_{i j} \int d x_{1} d x_{2} \hat{\sigma}(i j \rightarrow a b) f_{i}^{p}\left(x_{1}, \mu\right) f_{j}^{p}\left(x_{2}, \mu\right)
$$

where $\hat{\sigma}(i j \rightarrow a b)$ is the parton cross section for the reaction $i j \rightarrow a b$ and $i j$ runs over all the possible partons which can produce the partons $a$ and $b$ in the final state.

For the top quark production in $p \bar{p}$ collisions, we discuss only the strong production (2.29) because it dominates the top quark production at the Tevatron energy. The parton cross section $\hat{\sigma}$ is calculated by the perturbative expansion about the strong coupling constant $\alpha_{\mathrm{S}}$. In the lowest order $\left(\alpha_{\mathrm{S}}^{2}\right)$, the processes contributing to the heavy quark pair production are those of the gluon fusion and the quark annihilation:

$$
\begin{aligned}
& g+g \rightarrow Q+\bar{Q} \\
& q+\bar{q} \rightarrow Q+\bar{Q}
\end{aligned}
$$

The diagrams are shown in Figure 2.6. The kinematic consequences from the leading order calculation are 
- the heavy quark and antiquark are produced back-to-back in the parton-parton center of mass frame and remain back-to-back in a plane transverse to the colliding beam direction.

- the heavy quarks have an average transverse momentum of about $M_{Q} c$.

The inclusion of the higher order processes changes not only the value of the cross section but also the kinematics of the event. The heavy quark pair production cross section calculated by Nason, Dawson and Ellis [22] includes the following parton processes:

$$
\begin{array}{ll}
q+\bar{q} \rightarrow Q+\bar{Q} & \alpha_{\mathrm{S}}^{2}, \alpha_{\mathrm{S}}^{3} \\
g+g \rightarrow Q+\bar{Q} & \alpha_{\mathrm{S}}^{2}, \alpha_{\mathrm{S}}^{3} \\
q+\bar{q} \rightarrow Q+\bar{Q}+g & \alpha_{\mathrm{S}}^{3} \\
g+g \rightarrow Q+\bar{Q}+g & \alpha_{\mathrm{S}}^{3} \\
g+q \rightarrow Q+\bar{Q}+q & \alpha_{\mathrm{S}}^{3} \\
g+\bar{q} \rightarrow Q+\bar{Q}+\bar{q} & \alpha_{\mathrm{S}}^{3} .
\end{array}
$$

The theoretical calculation of the production cross section of the heavy quark has several uncertainties:

- higher order corrections

- correctness of the parton structure functions

- choice of the scale parameter $\mu$

- choice of the QCD parameter $\Lambda_{\mathrm{QCD}}$

Figure 2.7 shows the $\mu$ dependence of the $\sigma(p \bar{p} \rightarrow t \bar{t} X)$ for the leading order and next-to leading order calculations. The sensitivity to the scale $\mu$ is reduced with the inclusion of the $\mathcal{O}\left(\alpha_{\mathrm{S}}^{3}\right)$ corrections. Actually, the complete calculation to infinite order of $\alpha_{\mathrm{S}}$ should not depend on the arbitrary scale of $\mu$. Hence the effect from the higher order correction can be estimated by varying the scale parameter $\mu$. Ellis studied the total cross section 
for the top, bottom and charm production to the next-to-leading order [24] using the results of Nason et al. [22] with a recent set of the structure functions, DFLM [25]. The results for the $t \bar{t}$ production are shown in Figure 2.8. We find there is $20 \%(15 \%)$ uncetainty in the theoretical calculation of $\sigma(t \bar{t})$ for $M_{\text {top }}=80(200) \mathrm{GeV} / c^{2}$.

\subsection{Fragmentation of the Heavy Quark}

A bare quark has not been observed in the nature. The QCD gives a qualitative explanation for this reason. As a consequence of the non-abelian gauge group of the QCD, the effective coupling constant $\alpha_{S}$ becomes large at low energy, or at a long distance scale. Due to the large coupling constant, the strong field stores sufficient energy to create one or more quark-antiquark pairs from the vacuum until the initial quark-antiquark pair becomes apart. Then the initial quark (antiquark) together with the new created quarks forms a hadron. This "soft" process, called fragmentation or hadronization, cannot be calculated by the perturbative QCD. To describe the fragmentation process, the fragmentation function $D_{q}^{h}(z)$ which has to be determined experimentally is introduced. The fragmentation function $D_{q}^{h}(z) d z$ gives a probability that we see a hadron $h$ in the final particles, with an momentum fraction between $z$ and $z+d z$ of the parent quark $q$. The momentum fraction $z$ is defined as

$$
z=\frac{p_{\text {hadron }}}{p_{\text {quark }}}
$$

The fragmentation process of the heavy quark has been studied in $e^{+} e^{-}$experiments. It turned out that the fragmentation of the heavy quark was much different from that of the light quark. The distinctive feature is that a large fraction of the parent energymomentum is transferred to a heavy hadron. This kinematical characteristics of the heavy quark fragmentation is referred to as "hard" fragmentation. Peterson et al. gave an analytic form of the fragmentation function to account for the fragmentation process 
for the heavy quark $Q[26]$ :

$$
D_{Q}^{h}(z)=\frac{N}{z\left[1-(1 / z)-\epsilon_{Q} /(1-z)\right]^{2}}
$$

where $N$ is a normalization constant and $\epsilon_{Q}$ is a parameter of order $M_{q}^{2} / M_{Q}^{2}\left(M_{q}<1\right.$ $\left.\mathrm{GeV} / c^{2}\right)$. The fragmentation processes for the charm and the bottom quark were found to be well described by the Peterson fragmentation function [27] with the $\epsilon$ parameter given by

$$
\begin{aligned}
& \epsilon_{c}=0.06 \pm 0.03 \\
& \epsilon_{b}=0.006 \pm 0.003 .
\end{aligned}
$$

\subsection{Decay of the Heavy Quark}

In the approximation called "spectator model", the decay of a heavy hadron is described as the decay of a heavy quark contained in the hadron. Other constituents of the heavy hadrons are assumed to be free from the decay process. This approximation is valid when the released energy in the decay is much larger than the energy scale of the parton interaction in the hadron and is found to be good for describing the decay of the $b$ quark.

The decay of the top quark in the minimal Standard Model (with one neutral Higgs boson) is dominated by the process

$$
t \rightarrow W b
$$

Decays into the $W d$ and $W s$ are also allowed with the suppression due to the KobayashiMasukawa matrix element $V_{t d}$ and $V_{t s}$. The branching ratio of the top quark decay is given by counting the branching ratio of the $W$ decay. The real or virtual $W$ depending on the top quark mass succeedingly decays into 3 types of lepton pair ( $W \rightarrow l \nu, l=e$, $\mu$ and $\tau$ ) and 6 types of quark pair including the degree of 3 colors $\left(W \rightarrow q \bar{q}^{\prime}\right)$. Each 
channel has an approximately same branching fraction, $B r=1 / 9$.

In the case that the top quark mass is extremely heavy, the top quark decays before the fragmentation. Figure 2.9 shows the decay width of the top quark as a function of the top quark mass. Figure 2.10 shows the fraction that the top quark goes through the fragmentation process before the decay [28].

The decay products of the heavy quark usually have a large transverse momentum (a momentum projected onto the plane perpendicular to a colliding beam direction) because of the large energy released in the decay. Also these daughter particles tend to be well separated, or isolated, from each other. One can see this effect by simply considering the invariant mass of the daughters in a 3 body decay $Q \rightarrow q \ell \nu$ for instance:

$$
\begin{aligned}
M_{Q} & =\sqrt{\left(p_{q}+p_{\ell}+p_{\nu}\right)^{2}} \\
& =\sqrt{p_{q}^{2}+p_{\ell}^{2}+p_{\nu}^{2}+2 \sum_{i j}\left(p_{i} \cdot p_{j}\right)} \\
& =\sqrt{m_{q}^{2}+m_{\ell}^{2}+m_{\nu}^{2}+2 \sum_{i j}\left(E_{i} E_{j}-p_{i} p_{j} \cos \theta_{i j}\right)}
\end{aligned}
$$

The heavy mass $M_{Q}$ results in that the decay particles get more energetic and that they are separated from each other ( $\operatorname{small} \cos \theta_{i j}$ ).

Given the decay modes of the top quark and also given the distinctive properties of the top quark decay, the characteristics of the top quark event is the existence of isolated high $P_{\mathrm{T}}$ particles such as electrons, muons, neutrinos (missing energy), hadrons and high $E_{\mathrm{T}}$ jets.

\subsection{Top Search in Dilepton Events at CDF}

The signatures for the identification of the top quark production were discussed in the previous section. For the $t \bar{t}$ events produced in $p \bar{p}$ collisions, we can consider the following signatures: 
- high $P_{\mathrm{T}}$ multijet events

- high $P_{\mathrm{T}}$ isolated lepton plus jets events (lepton $\left.+j e t s\right)$

- high $P_{\mathrm{T}}$ dilepton events,

where the lepton refers to only the electron and muon. The $\tau$ lepton is not explicitly considered because the detection of the $\tau$ in $p \bar{p}$ collisions is not easy.

Although the multijet events where both the $t$ and $\bar{t}$ decay hadronically accounts for the largest branching fraction $36 / 81$ of all the top decay modes, it is difficult to distinguish the $t \bar{t}$ events from the copious ordinary QCD jet events.

Since leptons are identified reliably in $p \bar{p}$ collisions, a great improvement in the signal-to-background ratio is obtained by requiring a lepton in the event. This type of the mode lepton + jets accounts for the branching fraction of $24 / 81$. The most important background to the lepton $+j$ ets channel is the $W$ production with jets $(W+j e t s)$ followed by the leptonic decay of the $W$.

The dilepton channel where both the top quark and the antitop quark decay semileptonically provides the cleanest signature in $p \bar{p}$ collisions with, however, the smallest branching ratio of $4 / 81$. The analysis described in this thesis tries to search for the $t \bar{t}$ events in the high $P_{\mathrm{T}}$ dilepton channels including $e \mu$, ee and $\mu \mu$. The $e \mu$ channel, especially, has no significant background, while the $e e$ and $\mu \mu$ channels suffer from a large contribution of the Drell-Yan process $\left(p \bar{p} \rightarrow \gamma, Z \rightarrow l^{+} l^{-}\right)$. There is a small contribution involving $\tau$ 's, c's and b's which produce an electron or a muon in their decays. These processes are also included in the analysis. The charge combination of two leptons is not explicitly restricted. It can be a like-sign or an unlike-sign leptons resulting in a slight enhancement of the contribution from $b$ or $c$ decays. The detailed description of the analysis will be given in Chapters 4 and 5 . 


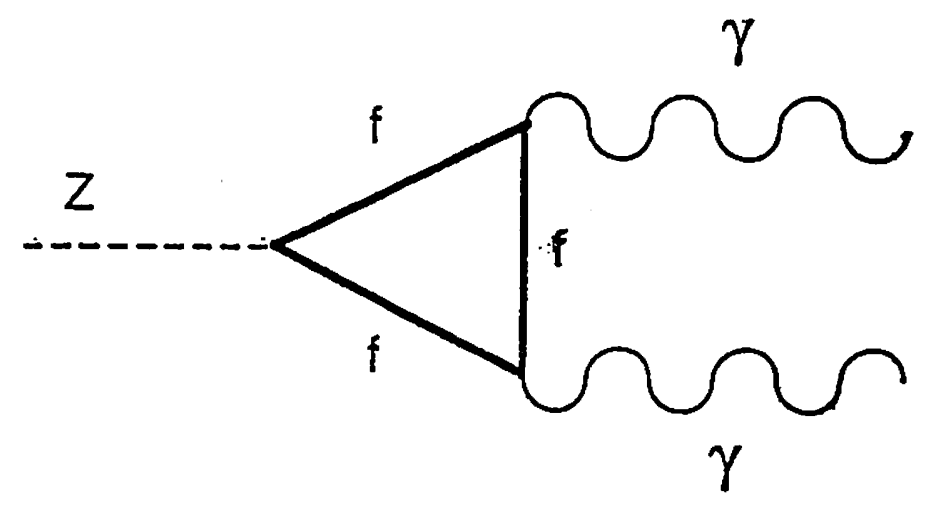

Figure 2.1: The $\gamma_{5}$ triangle diagram. 


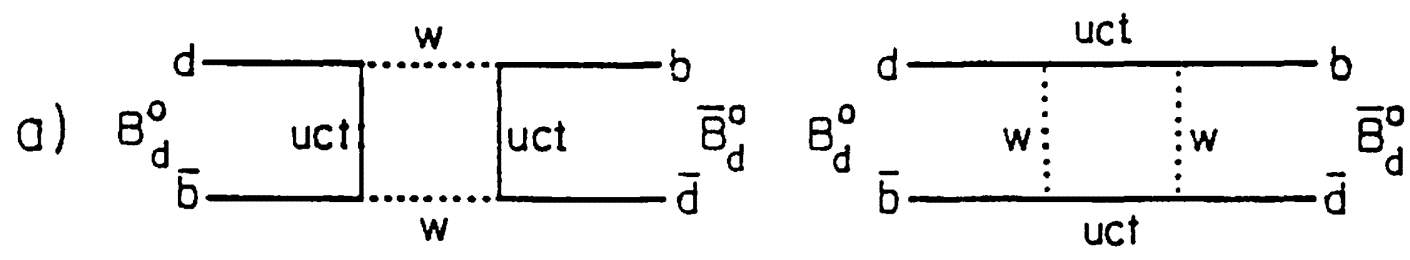

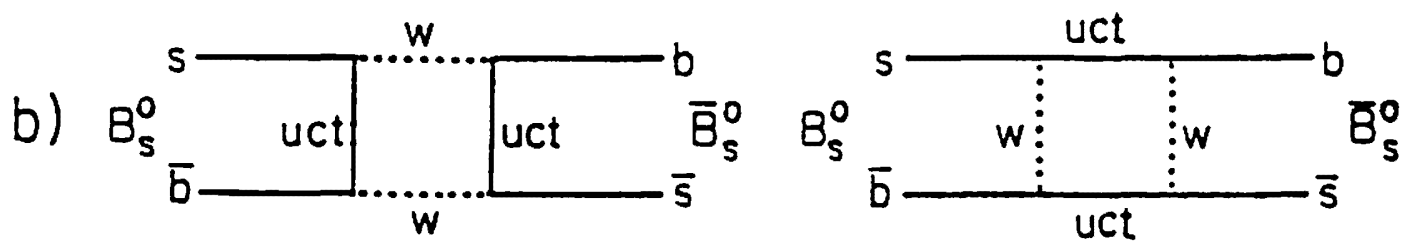

Figure 2.2: The box diagrams for (a) $B_{d}^{0}-\bar{B}_{d}^{0}$ and (b) $B_{s}^{0}-\bar{B}_{s}^{0}$ mixing. 

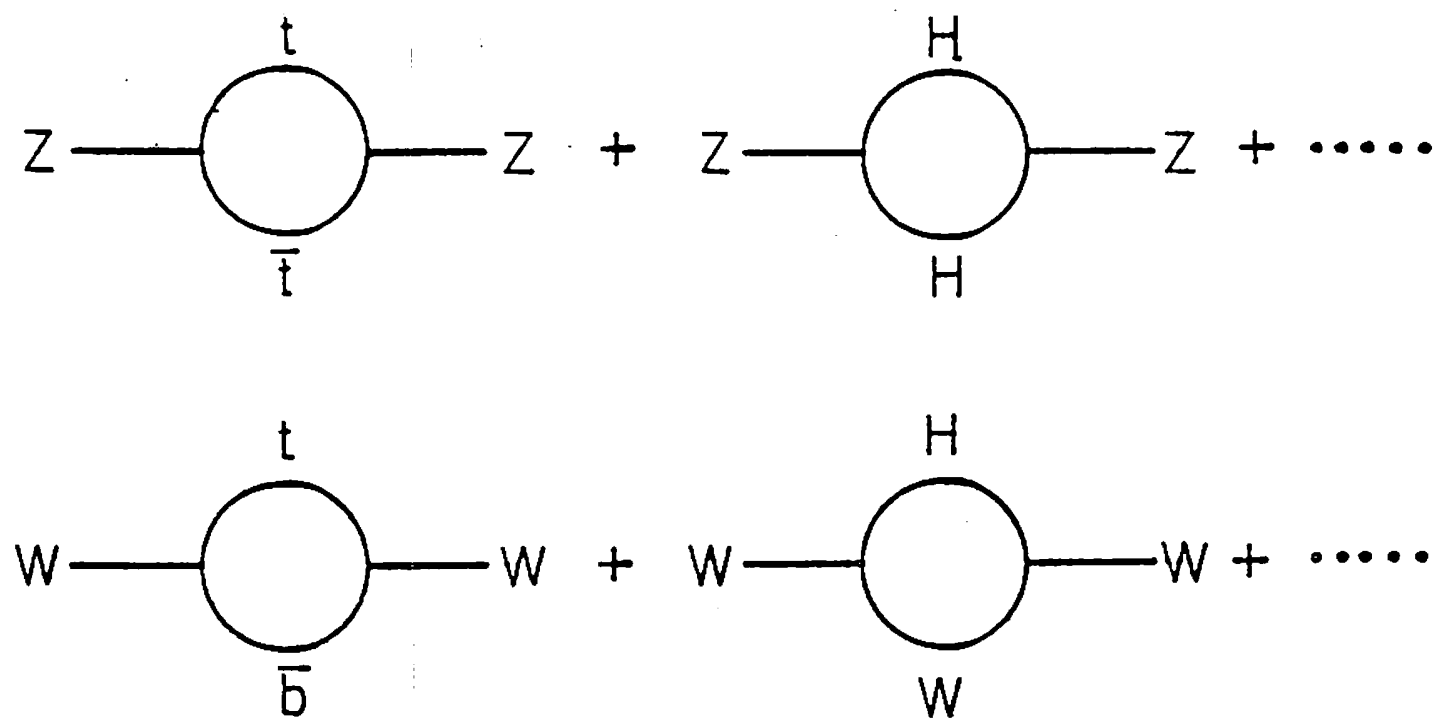

Figure 2.3: The diagrams for the radiative correction to the weak interaction. 


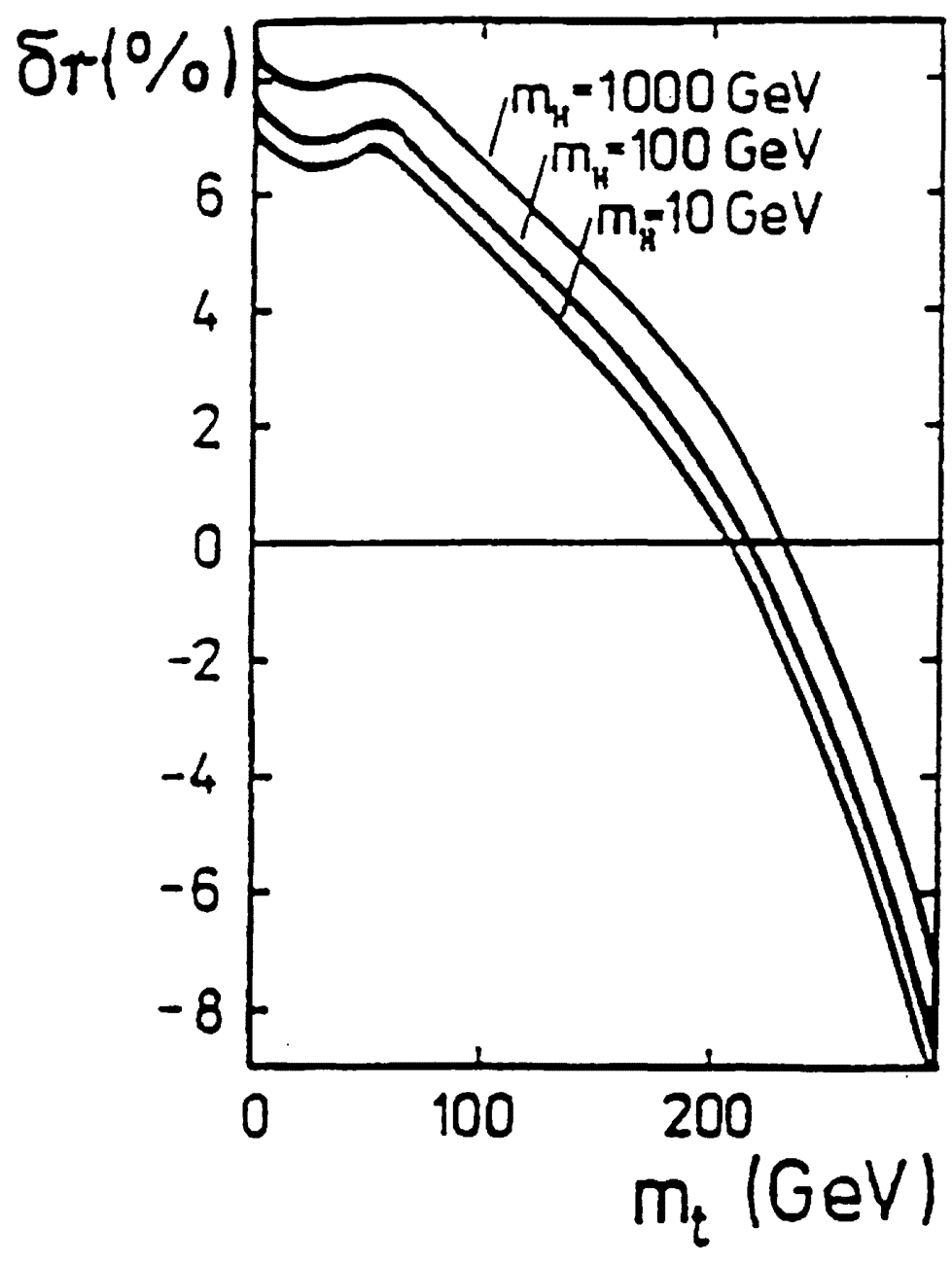

Figure 2.4: The radiative correction factor to the $W$ mass, $\Delta r$, as a function of. $M_{\text {top }}$ from Reference [10]. 


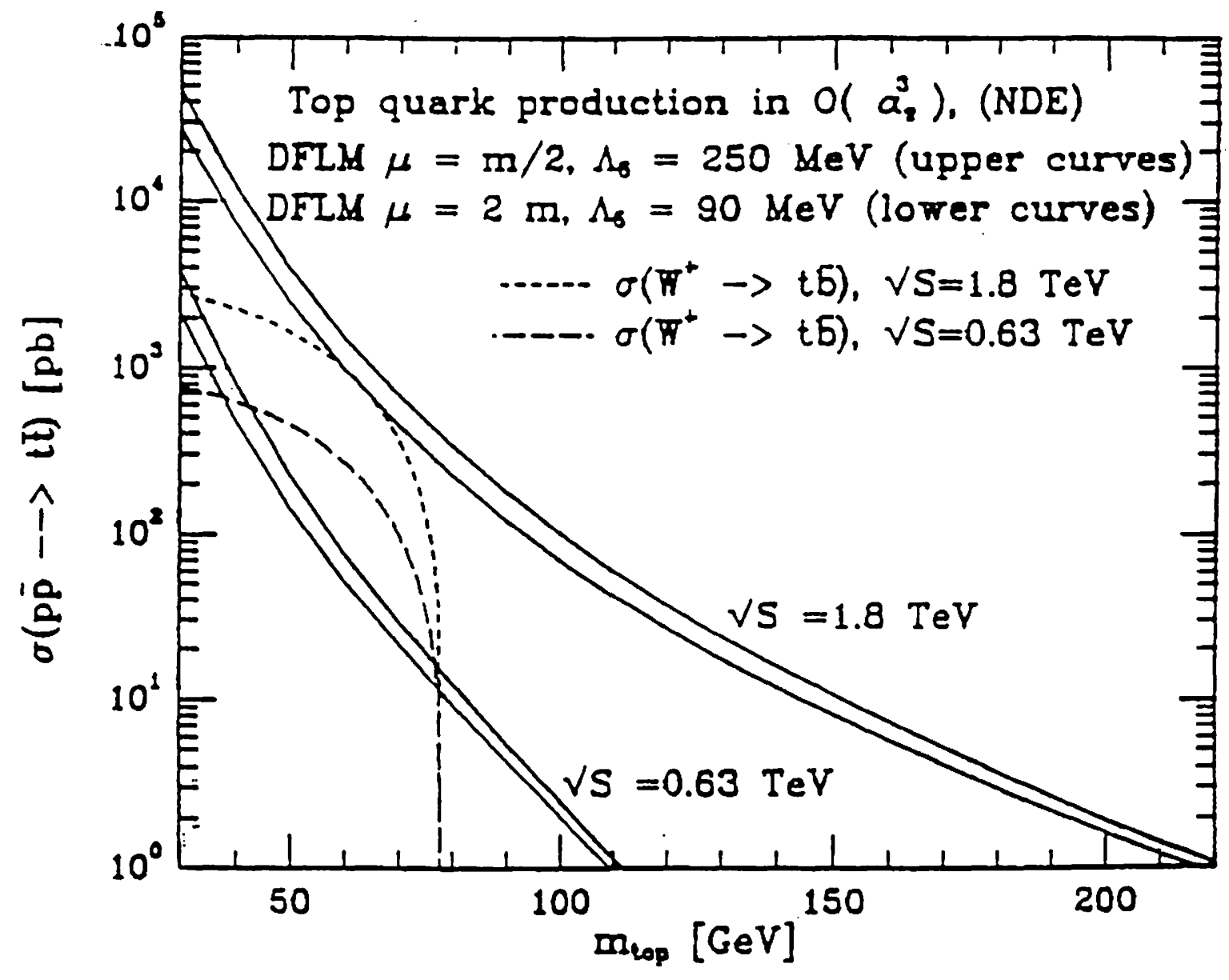

Figure 2.5: The $t \bar{t}$ production cross section in $p \bar{p}$ collisions by Altarelli et. al. [23], based on the next-to-leading order calculation by Nason, Dawson and Ellis [22]. 

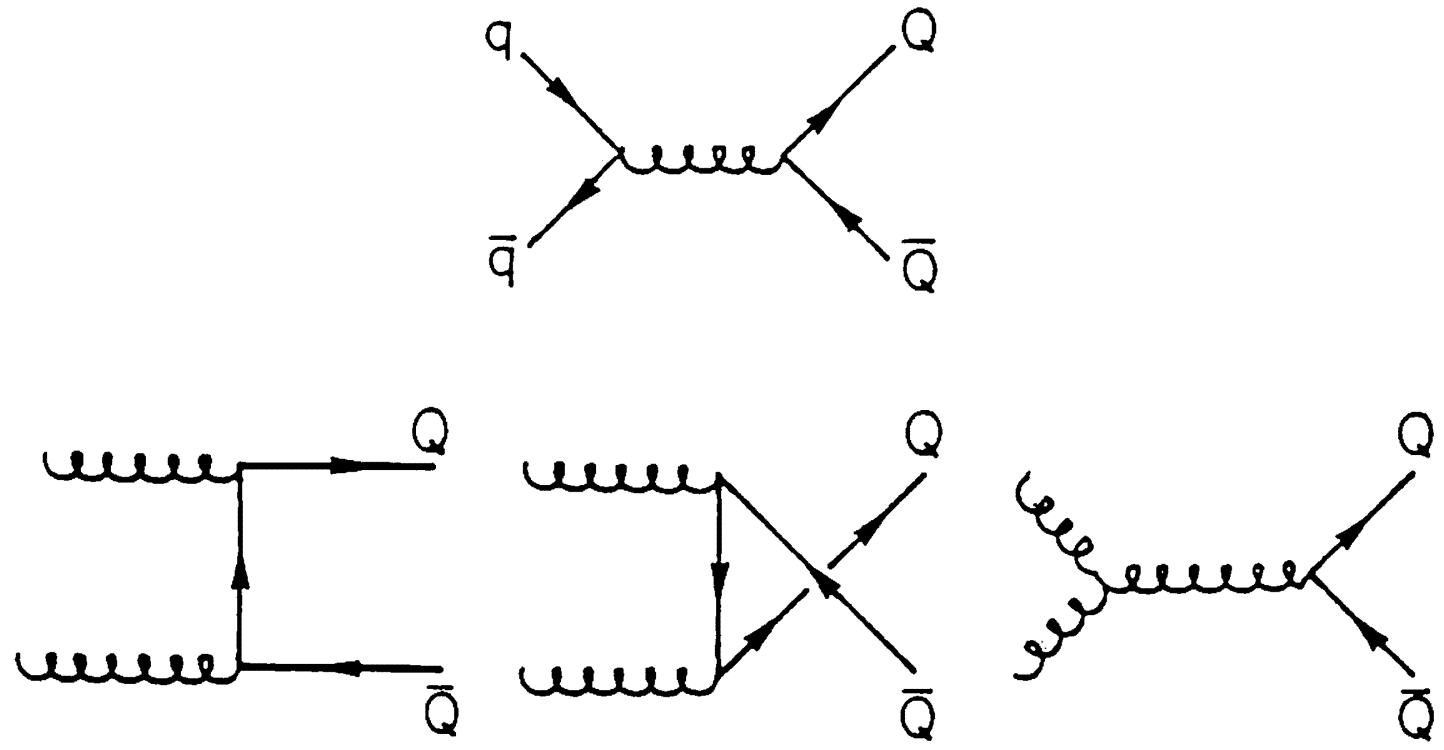

Figure 2.6: The lowest order Feynman diagrams for the heavy quark pair production. 


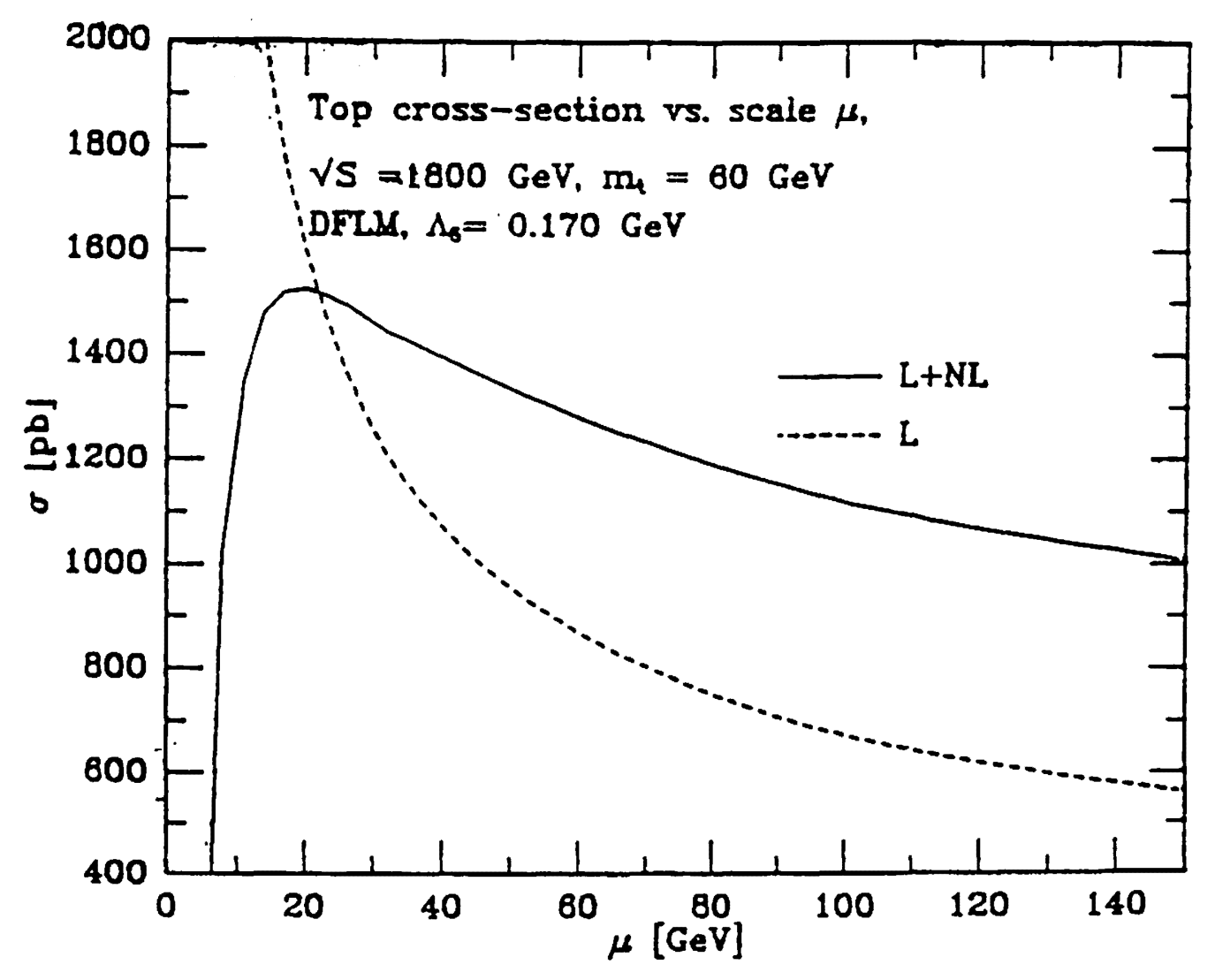

Figure 2.7: The $t \bar{t}$ production cross section as a function of the scale parameter $\mu$. 


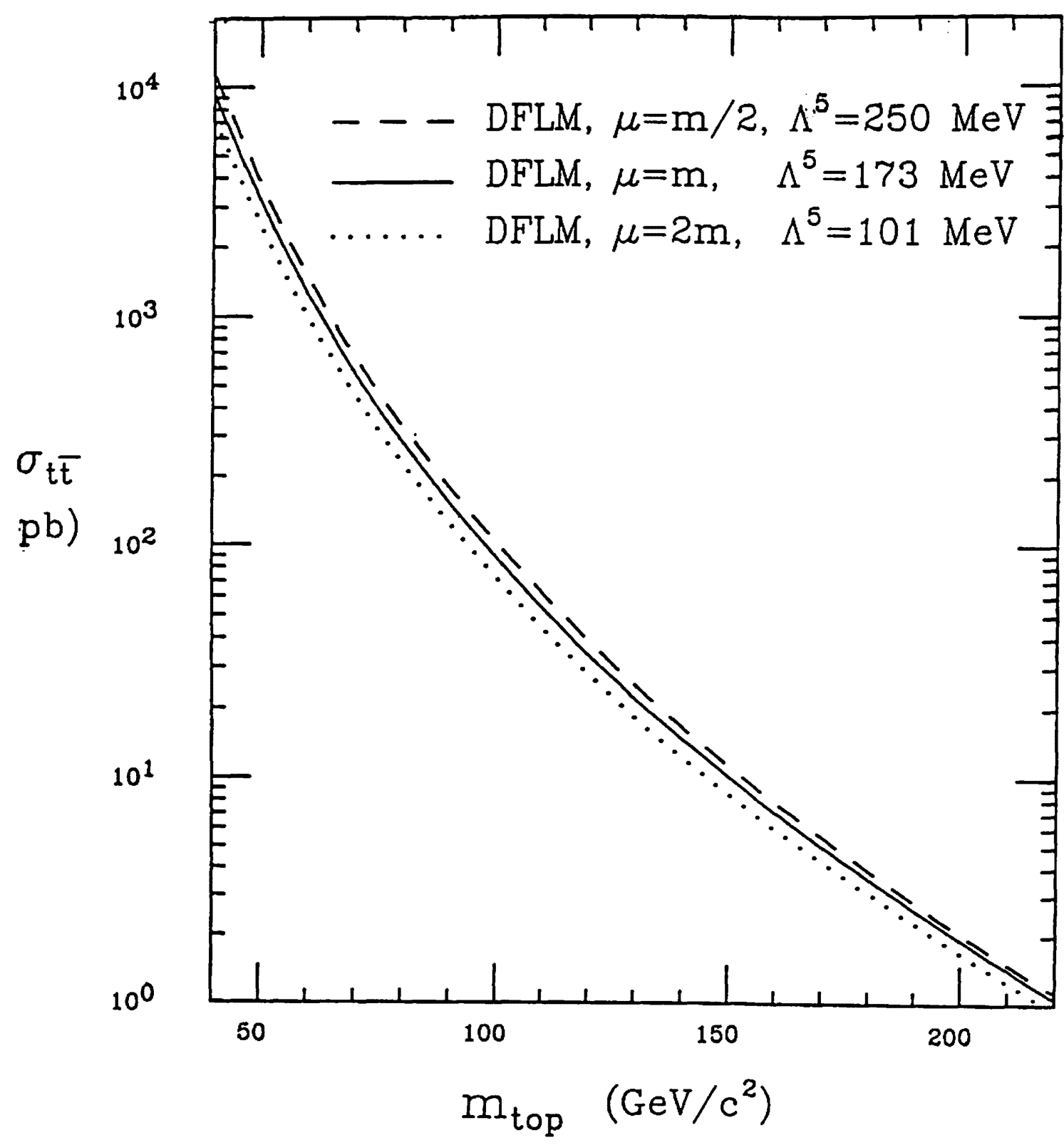

Figure 2.8: The $t \bar{t}$ production cross section at $\sqrt{s}=1.8 \mathrm{TeV}$ by Ellis [24], based on the next-to-leading order calculation by Nason, Dawson and Ellis [22]. 


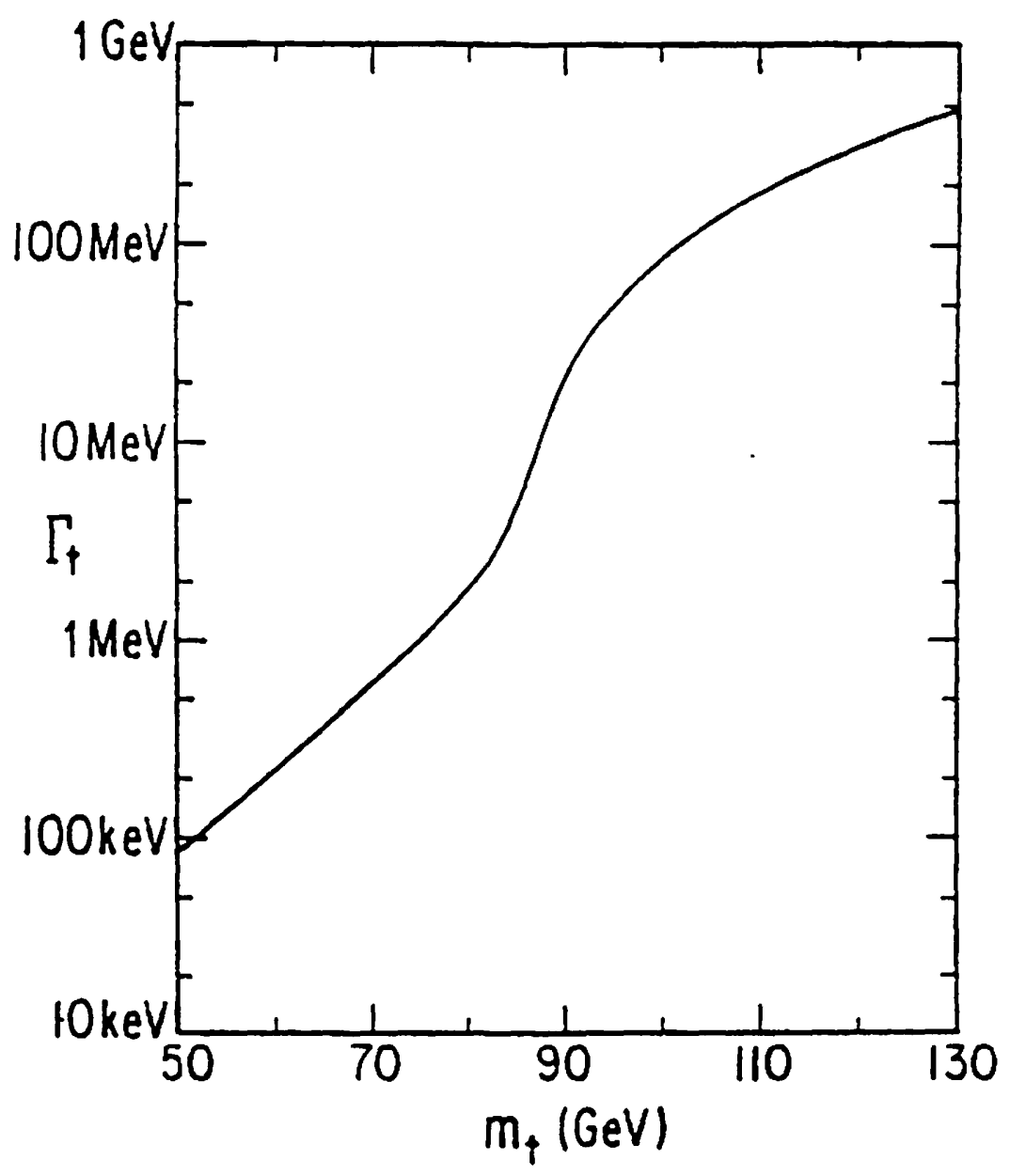

Figure 2.9: The decay width of the top quark as a function of the top quark mass. 


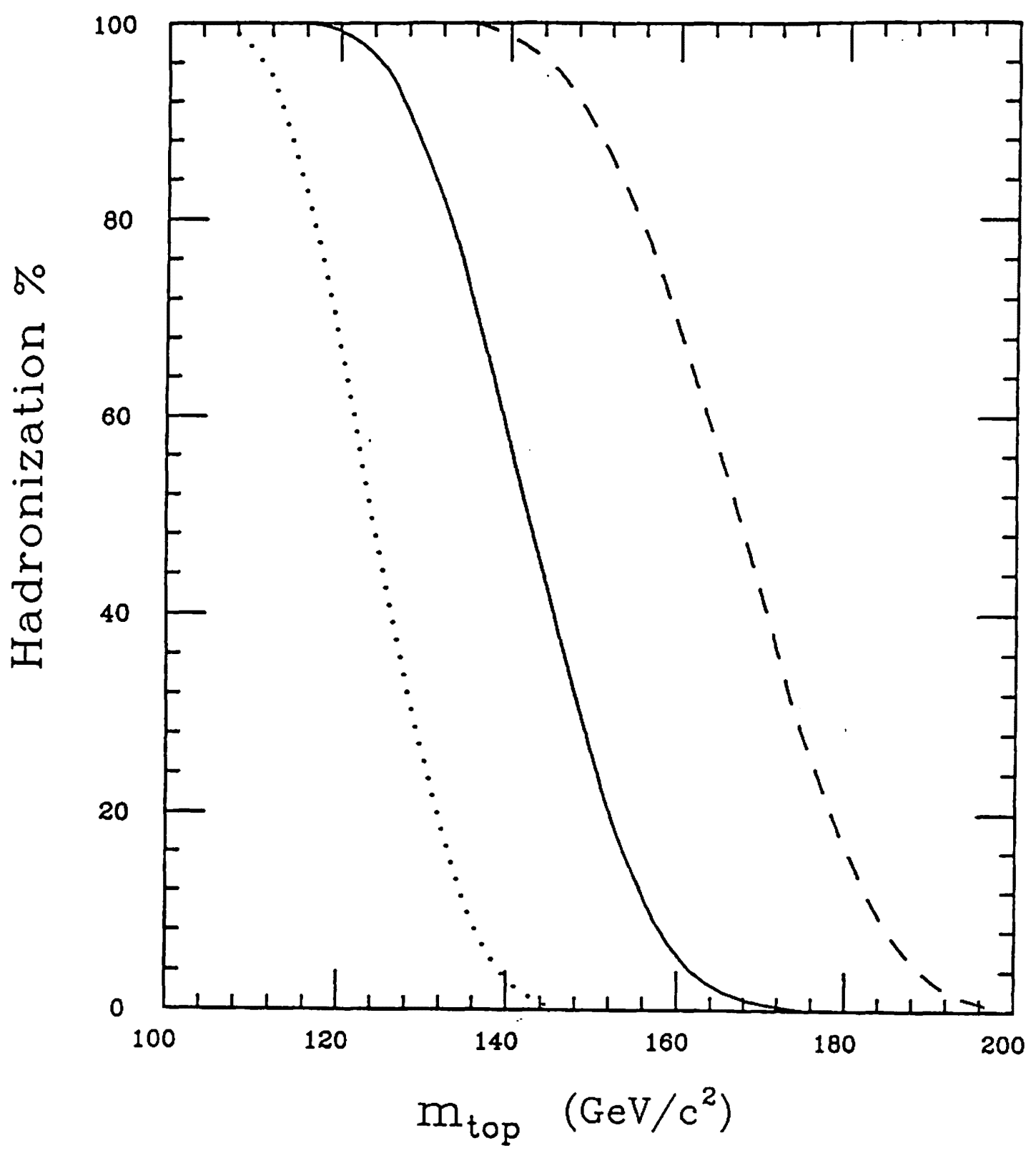

Figure 2.10: The fraction of the $t \bar{t}$ production for which the fragmentation occurs before the top quark decays, as a function of the top quark mass, for $1.8-\mathrm{TeV} p \bar{p}$ collisions. Solid line: hadronization scale $\Lambda^{-1}=1 \mathrm{fm}$. Dashed line: $\Lambda^{-1}=0.5 \mathrm{fm}$. Dotted line: $\Lambda^{-1}=2 \mathrm{fm}$. 


\section{Chapter 3}

\section{The CDF Detector}

\subsection{Detector Components}

The Collider Detector at Fermilab (CDF) was constructed in order to study $p \bar{p}$ collisions using the superconducting Tevatron collider at $\sqrt{s}=1.8 \mathrm{TeV}$. The CDF is located at one of the $p \vec{p}$ interaction regions, $\mathrm{B} 0$, along the Tevatron ring which has a diameter of $2 \mathrm{~km}$, and is the first detector which collected $p \bar{p}$ collision data at the highest center of mass energy currently available in the world.

The CDF is a general purpose detector covering most of the solid angle, full $2 \pi$ in azimuth and $2^{\circ} \leq \theta \leq 178^{\circ}$ with respect to the beam direction. It is capable of performing the reconstruction of charged particle tracks, the energy and momentum measurement and the identification of the particle species. The physical dimensions are, approximately, $30 \mathrm{~m}$ along the beam direction, $10 \mathrm{~m}$ in width and height. This large detector weighs 5000 ton in total. The CDF has about 100,000 electronic read out channels.

The CDF is azimuthally and forward/backward symmetric consisting of three major parts, the central detector and two identical forward/backward detectors. The central detector includes the superconducting solenoid providing 1.4 Tesla magnetic field for the momentum measurement of charged particles. Particles emerged from an interaction 
region encounter, in sequence, a thin wall beryllium beam pipe, tracking chambers, calorimeters and muon detectors. A perspective view of the CDF is shown in Figure 3.1. An elevation view of the CDF is shown in Figure 3.2.

The CDF coordinate system is defined as a right-handed system. The origin is taken at the center of the detector. The $z$ direction is defined along the proton beam, West to East, the $y$-axis points vertically upward and the $x$-axis points radially outward from the Tevatron ring. The polar angle $\theta$ and the azimuthal angle $\phi$ are defined in a usual way, that is, $\theta$ is measured with respect to the positive $z$-axis (the proton beam direction) and $\phi=0$ is along the positive $x$-axis with $\phi$ increasing toward the positive $y$-axis. The polar angle is quite often expressed in terms of the pseudo-rapidity $\eta=-\ln \left(\tan \frac{\theta}{2}\right)$. The central detector covers the polar angle region $10^{\circ}-170^{\circ}$ and the forward (backward) detector covers $2^{\circ}-10^{\circ}\left(170^{\circ}-178^{\circ}\right)$.

This chapter briefly describes the several components of the CDF detector which are relevant to the analysis presented in this thesis. The more complete descriptions are found in [29] and references therein.

\subsubsection{Beam-Beam Counter}

The Beam-Beam Counter (BBC) consists of two planes of scintillation counters located at the inner face $(|z| \sim 6 \mathrm{~m})$ of each of the forward and the backward detector. The $\mathrm{BBC}$ provides a trigger for $p \bar{p}$ inelastic scattering and monitors the beam luminosity. The angular coverage is $0.32^{\circ}-4.47^{\circ}\left(175.53^{\circ}-179.68^{\circ}\right)$ in the forward (backward) region. The structure of the BBC is shown in Figure 3.3.

\subsubsection{Tracking Detector}

\section{Vertex Time Projection Chamber}

The Vertex Time Projection Chamber (VTPC) system [30] consists of 8 modules arranged in a line along the beam direction surrounding the beam pipe of $5 \mathrm{~cm}$ diameter. The main purpose of the VTPC is the vertex measurement of a $p \bar{p}$ collision event by the 
reconstructing charged particle tracks. It covers $|\eta|<3.25$.

Each module of the VTPC is $35 \mathrm{~cm}$ long, and the total length is $2.8 \mathrm{~m}$. The inner radius and the outer radius is $7 \mathrm{~cm}$ and $21 \mathrm{~cm}$, respectively. Two modules are shown in Figure 3.4. Each module has an octagonal shape and the high voltage grid at the center divides the inner area into two $15 \mathrm{~cm}$ drift spaces. At the both ends of each octant, there are 24 anode sense wires and 24 cathode pads. The 24 sense wires are arranged in $R-\phi$ plane strung perpendicularly to both the radial direction and the beam direction to measure $R-z$ coordinates of tracks with the TDC read out. The 24 pads are arranged in three rows for $\phi$ measurements. Using these position information, the VTPC is capable of 3 dimensional track reconstruction.

At the Tevatron, the $p \bar{p}$ collision point fluctuates around the nominal position of $Z_{\text {event }}=0$ with $\sigma\left(Z_{\text {cvent }}\right) \simeq 35 \mathrm{~cm}$, which is well contained in the VTPC coverage. The VTPC determines the event vertex $Z_{\text {crent }}$ with a precision of $\sim 1 \mathrm{~mm}$. The electron identification algorithm also utilizes the VTPC track reconstruction by requiring the existence of the track associated with the electron candidate. The central tracking chamber has only its coverage up to $|\eta|<2.0$. Thus only the VTPC gives track information in $2.0 \leq|\eta| \leq 3.25$.

\section{Central Tracking Chamber}

The Central Tracking Chamber (CTC) [31] is a 3.2-m long cylindrical drift chamber surrounding the VTPC. It reconstructs the track of a charged particle in 1.4 Tesla axial magnetic field pointing to the $-z$ direction and measures the momentum. The coverage extends to $|\eta|<2.0$ but the best momentum measurement is possible in $|\eta|<1.0$.

The inner radius and the outer radius is $28 \mathrm{~cm}$ and $138 \mathrm{~cm}$, respectively. It has 84 layers of sense wires with TDC read out, which are gathered into 9 layers called "superlayers". The numbers 0-8 are assigned to them from the inner layer to the outer layer. Five superlayers contain 12 layers of the axial sense wires parallel to the beam direction and are used to measure $R-\phi$ coordinates. Four superlayers interleaved by 
the axial superlayers have 6 layers of the sense wires tilted by $\pm 3^{\circ}$ alternatively from layer to layer with respect to the beam direction to measure $R-z$ coordinates. They are called stereo superlayers. The wires in a superlayer are divided into the cells in $\phi$ direction. The number of cells changes from 30 for the innermost superlayer to 120 for the outermost superlayer, and there are 660 cells in total. The wires in a cell are tilted by $45^{\circ}$ from the radial direction to correct for the Lorentz angle of electron drift in the magnetic field. An endplate of the CTC is shown in Figure 3.5.

The momentum resolution degrades in large $|\eta|$ region where particles do not traverse all the superlayers. Particles in $|\eta|<1.0$ pass through all the superlayers and in this region the CTC system measures momenta of charged particles with the resolution of

$$
\frac{\Delta P_{\mathrm{T}}}{P_{\mathrm{T}}} \simeq 0.002 P_{\mathrm{T}}
$$

where $P_{\mathrm{T}}$ is the transverse momentum in $\mathrm{GeV} / c$. The best resolution

$$
\frac{\Delta P_{\mathrm{T}}}{P_{\mathrm{T}}} \simeq 0.0011 P_{\mathrm{T}}
$$

is obtained [32] for tracks which are constrained to come from the beam position in $R-\phi$ plane, which is offset a few $\mu \mathrm{m}$ from the nominal beam position $x=y=0$ and changes from store to store of beams.

The CTC also provides as a trigger for high $P_{\top}$ tracks.

\subsubsection{Calorimeter}

The particle energy is measured by the calorimeter. The CDF calorimeter consists of the electromagnetic (EM) calorimeter and the hadronic (HAD) calorimeter covering full $2 \pi$ in $\phi$ and $|\eta| \leq 4.2$. The CDF calorimeter systems are divided into several detectors according to the coverage in $\eta$. The central calorimeter covers $|\eta| \leq 1.1$, in which the HAD covers only $|\eta| \leq 0.9$. The plug calorimeter covers $1.1 \leq|\eta| \leq 2.4$, in which the HAD covers only $1.3 \leq|\eta| \leq 2.4$. The intermediate region between the central HAD 
and the plug $\mathrm{HAD}$ is covered by the endwall $\mathrm{HAD}$ calorimeter $(0.7 \leq|\eta| \leq 1.3)$. The forward/backward calorimeter extends from $|\eta|=2.2$ to 4.2 while the HAD covers from 2.3 to 4.2. A part of the forward/backward EM calorimeter is followed by the plug HAD calorimeter. They all have a projective tower geometry with fine granularity in $\eta$ - $\phi$ common to both the EM and the HAD calorimeter.

\section{Central Calorimeter}

The central calorimeter is located outside the solenoid. The EM calorimeter occupies from $R=1.7 \mathrm{~m}$ to $2.0 \mathrm{~m}$, which is followed by the $\mathrm{HAD}$ calorimeter up to $R=3.5$ $\mathrm{m}$. The overall dimension is $4.9 \mathrm{~m}$ along the beam direction. The central calorimeter is divided into two parts, east side and west side, which face each other at $\eta=0$ where a small inactive region is left $\left(90^{\circ} \mathrm{crack}\right)$. Each part is further segmented to 24 modules in $\phi\left(\Delta \phi=15^{\circ}\right)$. Each module forms a single unit called wedge, together with the muon detector behind the HAD calorimeter. The 24 wedges are assigned numbers from 0 to 23 starting from $\phi=0$. Out of 24,12 wedges make a semi-circle called arch. Two arches touch each other at $\phi=90^{\circ}$ and $270^{\circ}$ so as to surround the inner part of the central detector. The calorimeters in a wedge have projective tower geometry in $\eta, 10$ towers for EM calorimeters (numbered 0-9 from small $|\eta|$ ) and 9 towers $(0-8)$ for HAD calorimeters. The resulting segmentation of the central calorimeter is $\Delta \eta \times \Delta \phi \simeq 0.1 \times 15^{\circ}$. The physical dimension of a tower is approximately $24 \mathrm{~cm}$ in $z$ by $45 \mathrm{~cm}$ in $\phi$ direction at the bottom of the EM calorimeter, and $28 \mathrm{~cm}$ by $56 \mathrm{~cm}$ at the bottom of the HAD calorimeter. One of 48 wedges is shown in Figure 3.6. The tower geometry of the central calorimeter is shown in Figure 3.7.

The central EM calorimeter (CEM) [33] consists of 31 layers of $5 \mathrm{~mm}$ thick scintillator and 30 layers of $1 / 8$ inch lead absorber arranged alternately. The thickness is $\simeq 18 X_{0}$ (radiation length) including the solenoid. Using the test beam electron with an energy 
range of $15-100 \mathrm{GeV}$, the energy resolution of the CEM was measured [33] as

$$
\frac{\Delta E}{E}=\frac{13.5 \%}{\sqrt{E \sin \theta}}
$$

where the $\sin \theta$ reflects the change of the sampling thickness seen by the electron emitted at polar angle $\theta$.

The CEM has a gas proportional wire chamber with cathode strips (CES) embedded at the approximate EM shower maximum ( $\simeq 5.9 X_{0}$ including the solenoid). The CES consists of the wires along the beam direction for $\phi$ measurements and cathode strips perpendicular to the wires for $z$ measurements. The CES provides a precise determination of the shower center and the shower shape. The position resultion is about $2.2 \mathrm{~mm}$ in the wire $(\phi)$ view and $1.4 \mathrm{~mm}$ in the strip view for $50 \mathrm{GeV} / c$ electrons.

The central HAD calorimeter (CHA) [34] consists of sandwiches of $2.5 \mathrm{~cm}$ thick iron and $1 \mathrm{~cm}$ thick scintillator. The thickness of the CHA is $4.5 \lambda$ (absorption length). The energy resolution is given by

$$
\frac{\Delta E}{E}=\frac{75 \%}{\sqrt{E \sin \theta}}
$$

from the test beam pion data with an energy range of 10-150 GeV [34].

\section{Plug Calorimeter}

The plug calorimeter [35] is located at the end of the CTC and consists of the EM calorimeter (PEM) and HAD calorimeter (PHA) behind.

The PEM has a cylindrical structure occupying from $|z|=1.7 \mathrm{~m}$ to $2.3 \mathrm{~m}$ with the outer radius of $1.4 \mathrm{~m}$ and is surrounded by the solenoid. The inner radius varies from 30 $\mathrm{cm}$ to $39 \mathrm{~cm}$ as $|z|$ increases. The PEM is divided into 4 quadrants in $\phi$ direction, leaving a small inactive region around every $90^{\circ}$ boundary in $\phi$. A cross sectional view of the PEM is shown in Figure 3.8 and an isometric view of a quadrant is shown in Figure 3.9. The PEM consists of 34 sampling layers arranged perpendicularly to the beam direction, where each layer includes a lead panel as an absorber and a layer of gas proportional 
wire chambers with cathode pads as an active medium. The total thickness of the PEM is $18-21 X_{0}$. These layers are divided into 3 groups in $z$ direction, the first and the last 5 layers form the first and the third segment and the rest of 24 layers form the second segment. The energy deposited in the second segment is used in the trigger. The cathode pad panel is physically segmented into 16 channels in $\eta$ and 18 channels in $\phi\left(\Delta \phi=5^{\circ}\right)$. The $\eta$ width of the pad varies along $\eta(\Delta \eta=0.05-0.1)$. The segmentation used in the analysis is slightly different. At the outermost pad (the smallest $|\eta|$ in the plug region), the three pads along $\phi$ are grouped to form a tower of the same $\eta-\phi$ segmentation as in the central, $\Delta \eta \times \Delta \phi=0.1 \times 15^{\circ}$. Next two pads in $\eta$ and three pads in $\phi$ form a single tower of the same $\Delta \eta, \Delta \phi$ as in the central. In $1.32 \leq|\eta| \leq 1.41$, two pads in $\eta$ are combined to have a standard plug tower size, $\Delta \eta \times \Delta \phi=0.09 \times 5^{\circ}$. Each of other pads forms one tower by itself with a standard plug tower size.

The energy resolution was measured in the electron test beam with an energy range of $20-200 \mathrm{GeV}[35]$ as

$$
\frac{\Delta E}{E}=\frac{28 \%}{\sqrt{E}}
$$

The PHA has a truncated cone shape extending from $|z|=2.3 \mathrm{~m}$ to $3.7 \mathrm{~m}$. The PHA consists of 20 sampling layers of a steel plate and gas proportional wire chambers with cathode pads. The thickness is $\simeq 5.7 \lambda_{0}$. Unlike the PEM, the PHA is divided at every $30^{\circ}$ in $\phi$. Each part, called stack, has 12 pads in $\eta$ and 3 pads in $\phi$. The resulting pad segmentation is the same as in the PEM, $\Delta \eta \times \Delta \phi=0.09 \times 5^{\circ}$. The longitudinal 20 samples are ganged into a projective tower.

The energy resolution for the PHA was measured as

$$
\frac{\Delta E}{E}=\frac{132 \%}{\sqrt{E}}
$$

by the test beam pions with an energy range of $40-200 \mathrm{GeV}[36]$. 


\section{Wall Hadron Calorimeter}

The wall hadron calorimeter (WHA) [34] is instrumented in the uncovered region between the CHA and PHA. The WHA is attached to the central wedge at the side end of it $(|z|=2.5 \mathrm{~m})$. The overall dimension is $80 \mathrm{~cm}$ along the $z$ direction and $1 \mathrm{~m}$ along the radial direction. A side view of the one module is shown in Figure 3.7.

The WHA has a same structure as the central wedge in $\phi\left(\Delta \phi=15^{\circ}\right)$. It contains 6 towers with the same $\Delta \eta, \Delta \phi$ as in the CEM or the CHA, and consists of 15 layers of $5 \mathrm{~cm}$ steel and $1.0 \mathrm{~cm}$ scintillator. The thickness is $\simeq 5.7 \lambda_{0}$. The physical tower size is $25 \mathrm{~cm}$ along the radial direction from the beam axis and $35 \mathrm{~cm}$ along the $\phi$ direction at the bottom of the tower.

The energy resolution for the WHA is measured as

$$
\frac{\Delta E}{E}=\frac{80 \%}{\sqrt{E}}
$$

from the test beam pion data with an energy range of 10-150 GeV [34].

\section{Forward/Backward Calorimeter}

The forward/backward calorimeters [37] consist of layers of gas proportional sampling chambers interleaved with lead for the EM calorimeter (FEM) and steel for the HAD calorimeter (FHA). These calorimeters are not directly used in this analysis but contribute in the calculation of missing $E_{\Gamma}$ which is obtained from the calorimeter energy imbalance up to $|\eta|=3.6$.

\subsubsection{Central Muon Detector}

The central muon detector (CMU) [38] is mounted in the outermost part of the central detector. It detects muons having traversed approximately 5 absorption lengths of material in $|\eta|<0.63$ and also provides a trigger for muons.

The CMU is built into each wedge with the bottom of the chamber $3.5 \mathrm{~m}$ away from 
the beam line. The chambers are $2.3 \mathrm{~m}$ along the beam line. The CMU chambers in a wedge subtend $12.6^{\circ}$ in $\phi$, leaving a gap in the central muon coverage of $2.4^{\circ}$ between each wedge. The CMU is further segmented in $\phi$ into three modules of $\Delta \phi=4.2^{\circ}$ each of which is bolted together to form a single unit. The layout of the central muon chambers in one wedge and the arrangement of the chamber planes are shown in Figures 3.10 and 3.11 , respectively.

Each of the $\Delta \phi=4.2^{\circ}$ modules consists of 4 layers of 4 rectangular drift cells. A stainless steel sense wire is strung at the center of the cell. The 4 sense wires, one from each layer, make up a muon tower. Alternating two of the 4 sense wires lie on a radial line which pass through the beam axis in $R$ - $\phi$ plane. Other 2 wires are $2 \mathrm{~mm}$ offset from this line. This resolves which side of the sense wires (in $\phi$ ) a track passes. The angle $\alpha$ between a track and the radial line can be determined by measuring the drift time difference of arriving electrons. With a charge division, $z$ coordinate is also measured.

The angle measurement is translated to the transverse momentum measurement. We denote by $D$ the distance from the beam axis to the crossing point of a particle track and the radial line. The $\alpha$ is related to $P_{r}$ by

$$
\sin \alpha=\frac{q B L^{2}}{2 D P_{\Upsilon}}
$$

where $B$ is the magnitude of the magnetic field, $L$ is the radius of the solenoidal magnetic field [38]. Putting $B=1.4116 \mathrm{~T}, L=1.440 \mathrm{~m}$ and $D=3.470 \mathrm{~m}$ (the bottom of the muon chambers) in Equation (3.8), we have

$$
\sin \alpha(\simeq \alpha)=\frac{126(\mathrm{mrad} \cdot \mathrm{GeV} / c)}{P_{\uparrow}}
$$

for $q=e=1.6 \times 10^{-19} \mathrm{C}$. The approximation $\sin \alpha \simeq \alpha$ is good already for $P_{\mathrm{T}}=1$ $\mathrm{GeV} / c$. The $P_{\text {[ }}$ measurement is degraded due to multiple scattering of a particle in the calorimeter materials. The average uncertainty in the angle $\alpha$ due to the multiple 
scattering [39] is represented as

$$
\Delta \alpha=\frac{85(\mathrm{mrad} \cdot \mathrm{GeV} / \mathrm{c})}{P_{\mathrm{\Gamma}}}
$$

Therefore the momentum resolution of the CMU is

$$
\frac{\Delta P_{\mathrm{T}}}{P_{\mathrm{T}}} \simeq \frac{\Delta \alpha}{\alpha} \sim 67 \%
$$

The uncertainty in the angle measurement due to the finite position resolution $(\sim 250$ $\mu \mathrm{m})$ is negligible small compared to that due to the multiple scattering process.

\subsection{Trigger System}

In the 1988-1989 run, the Tevatron was operated at a typical luminosity of $10^{30} \mathrm{~cm}^{-2} \mathrm{~s}^{-1}$ in six bunch mode ( 6 proton bunches and 6 antiproton bunches). The beam crossing interval was $3.5 \mu \mathrm{s}(286 \mathrm{kHz})$. The cross section of the inelastic $p \bar{p}$ interaction at 1.8 $\mathrm{TeV}$ is about $47 \mathrm{mb}$ resulting in a rate of $40-50 \mathrm{kHz}$. In order to reduce this high rate to a manageable rate of $\sim 1 \mathrm{~Hz}$ at which the data can be written to a tape, and to be preferentially sensitive to events of physics interest, the CDF employed four layers of triggers called level 0-3. At Level-1 and level-2 trigger [40], hardware trigger processors operate on the various information from the CDF detector. The event readout is initiated after level 2 accepts an event. Then digitized data is sent to level 3 trigger [41] for more sophisticated selection.

\section{Level 0}

Level-0 trigger requires a coincidence of hits between the east and west BBC counter to select inelastic $p \bar{p}$ interactions. A decision is made within $3.5 \mu$ s (no dead time). If level 0 accepts an event, it is inhibited during the next beam crossing. Thus $7 \mu$ s dead time is incurred. A typical output rate of level 0 was $\sim 50 \mathrm{kHz}$. 


\section{Level 1}

Level-1 decision which takes less than $3.5 \mu \mathrm{s}$ is based on the global features of calorimeter energy deposition, high $P_{\mathrm{T}}$ CTC track and hits in the muon detectors. The calorimeter segmentation is preserved at a coarser granularity than the offline towers, and called trigger towers. Each trigger tower is formed by adding several offline towers so as to have a common size of $\Delta \eta \times \Delta \phi=0.2 \times 15^{\circ}$. The electromagnetic transverse energy, the hadronic transverse energy, the total transvers energy which are summed over trigger towers with $E_{\mathrm{T}}$ above programmable thresholds, and the $E_{\mathrm{T}}$ imbalance are used for a decision. The Central Fast Tracker (CFT) [42] identifies high $P_{\mathrm{T}}$ tracks by comparing observed axial hits in the CTC with the predetermined hit patterns in a look-up table stored in a memory. No information on $\phi$ direction is available. The central muon trigger can measure the $P_{\uparrow}$ of a muon candidate as described in $\$ 3.1 .4$. Level 1 reduces level-0 output to a few $\mathrm{kHz}$.

\section{Level 2}

Given the level-1 delivery rate, Level 2 is allowed approximately $10 \mu \mathrm{s}$ for its decision. In this time, it is possible to form clusters of energy, to calculate the cluster position and width, to match a CTC track to an energy in the calorimeter cluster or to hits in the muon detector. Thus level 2 is able to do a first-order identification of physics objects such as electrons, photons, muons and jets. The level-2 triggers include jet, photon, electron, muon, missing $E_{\mathrm{T}}$, dielectron, diphoton, dimuon and $e \mu$ triggers. Level 2 reduces the event rate to less than $100 \mathrm{~Hz}$. If level 2 accepts an event, event readout starts, which takes $\sim 1 \mathrm{~ms}$.

\section{Level 3}

When an event satisfies level 2, the event data are digitized and read out by scanner modules. During the data are transferred to level 3 , they are formatted into the standard 
CDF data format by one of the data acquisition (DAQ) FASTBUS modules. Level 3 consists of a farm of 60 parallel micro-processors which are developed by Fermilab Advanced Computer Program (ACP) [43]. Each ACP node has a power equivalent to about $67 \%$ of a VAX 780 . The same FORTRAN codes as used in offline analysis run on it for more sophisticated event reconstruction and selection.

\subsection{Data Acquisition System}

The CDF Data AcQuisition system (DAQ) [44] employed a FASTBUS-based multi-level network. It reads the data with a typical size of $\sim 100 \mathrm{kbytes}$ for one $p \bar{p}$ collision event from $\sim 100,000$ electric channels.

The CDF DAQ has multiple partitions each of which covers one or more independent detector sections so that the DAQ for the different detector components can proceed in parallel. At the lowest level of the DAQ system, there are two major types of frontend systems. All the calorimeters and the central muon chambers use the Redundant Analog Bus-Based Information Transfer (RABBIT) system developed at Fermilab [45]. This crate-based system consists of analog charge amplifire cards with sample-and-hold circuits (RABBIT cards), a digitizer called Event Write Encoder (EWE module) with a capability of the analog pedestal subtraction and the test-over-threshold, and a scanner module (MX) interfaced to FASTBUS via the Multiple Event Port (MEP). Most tracking detectors use commercial FASTBUS TDCs read by the scanner module SSPs [46]. These front-end electronics except the scanner modules are located near the corresponding detectors, while the scanner modules are in the CDF control room. Each of the scanners can buffer 4 events.

The operation of the scanner modules and the trigger system are controlled by the Trigger Supervisor (TS) module [47]. When the level-2 trigger accepts an event, the Trigger Supervisor directs the scanner modules to read the data from the front-end electronics. After all the scanning has completed, the Trigger Supervisor sends a FASTBUS 
message to BuFfer Manager (BFM) indicating that an event is available in a specified buffer while the TS initiates the trigger process again unless all the four scanner buffers are full. The Buffer Manager supervises the dataflow from the scanner modules to the host VAX computer. Receiving the FASTBUS message from the Trigger Supervisor, the Buffer Manager directs the EVent Builder (EVB) module [48] to "Pull" an event from a specified buffer in all the scanner modules. The Event Builder is a group of FASTBUS modules which read, buffer and reformat a complete event from any allowed partition of the detector components. When the Event Builder has finished reading, it sends back a "Pull OK" message to the Buffer Manager and the BFM in turn notifies the Trigger Supervisor that the specified buffer in a scanner is available for the next reading process while the BFM directs the EVB to write a complete event into a specified node in the level-3 processor farm (ACP) [43]. Accepted events by the level-3 trigger are read by the buffer multiplexor executing on one or more computers in the VAX cluster. 


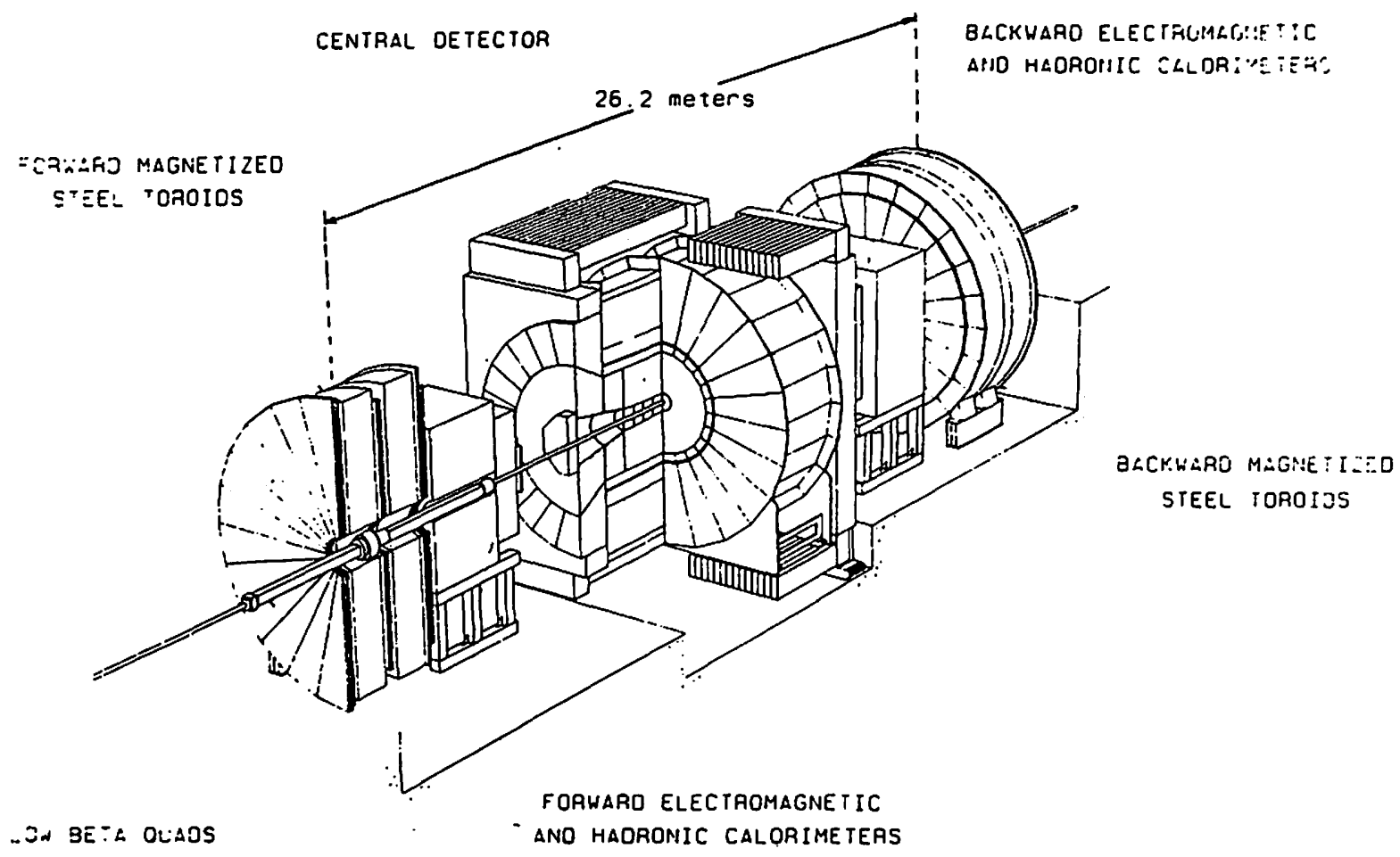

Figure 3.1: A perspective view of the CDF detector showing the central detector and the forward and backward detectors. 


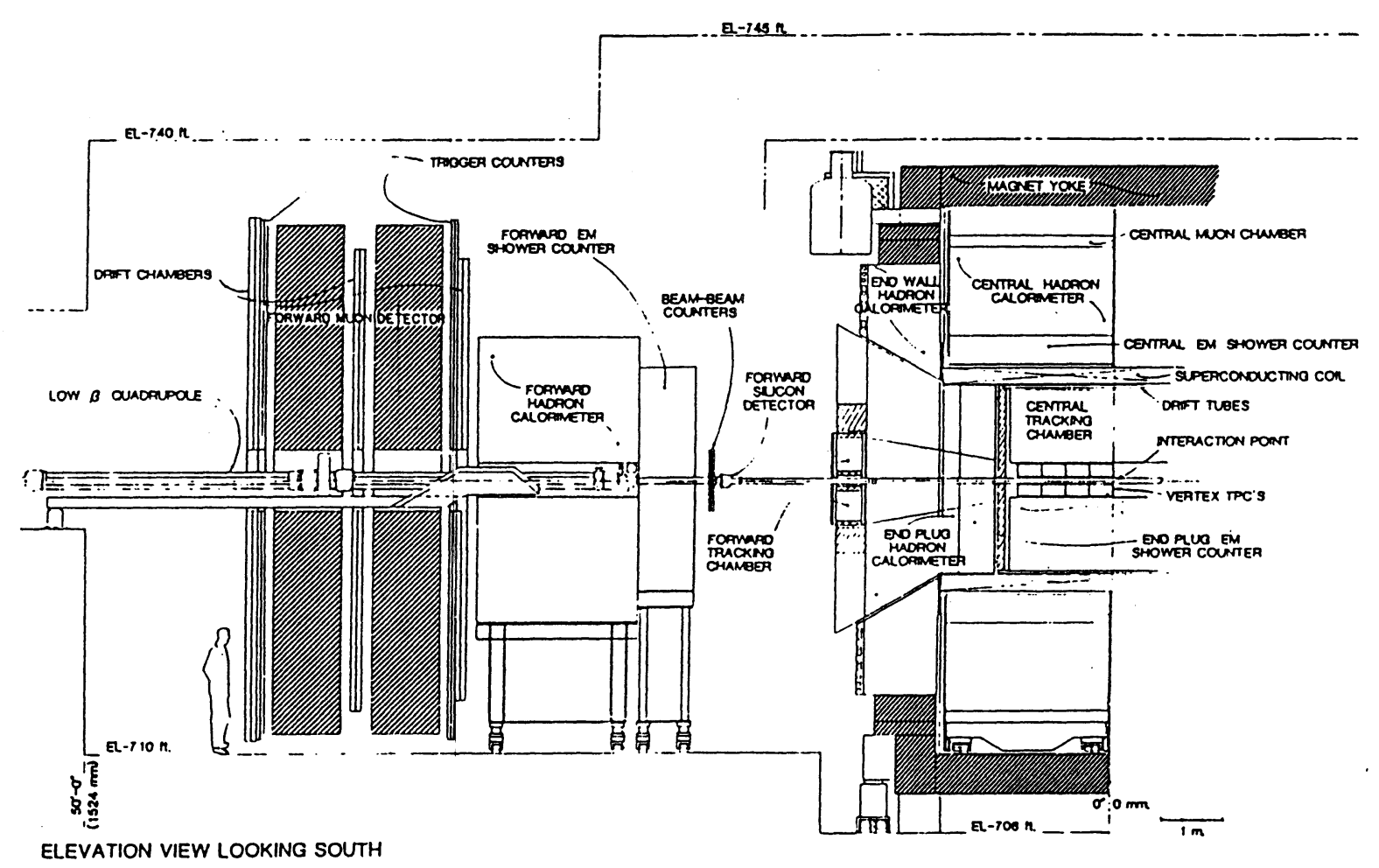

Figure 3.2: An elevation view through the forward half of the CDF. The detector is forward-backward symmetric about the interaction point. 


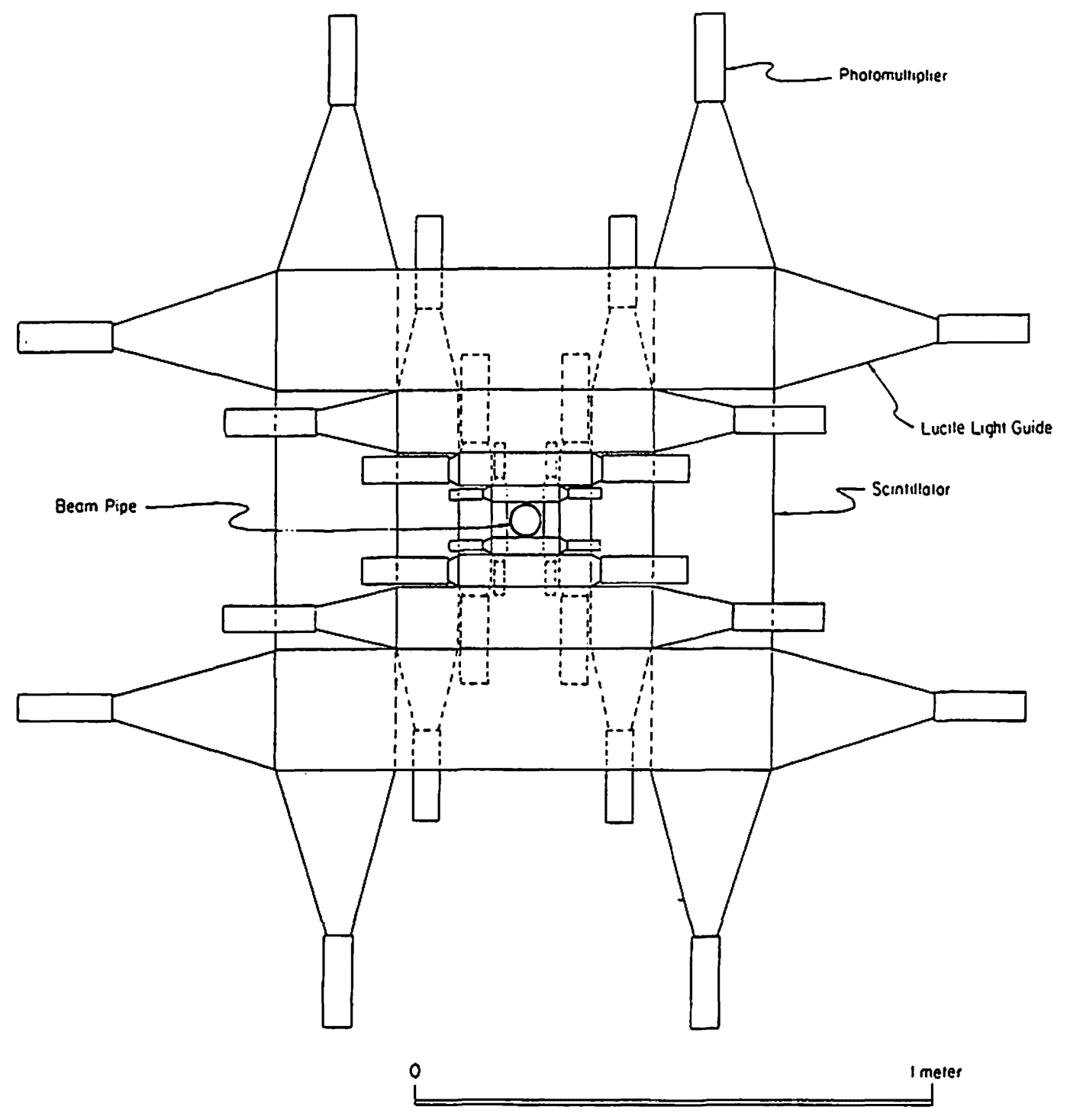

Figure 3.3: A beam's-eye view of one of the beam-beam counter planes. 


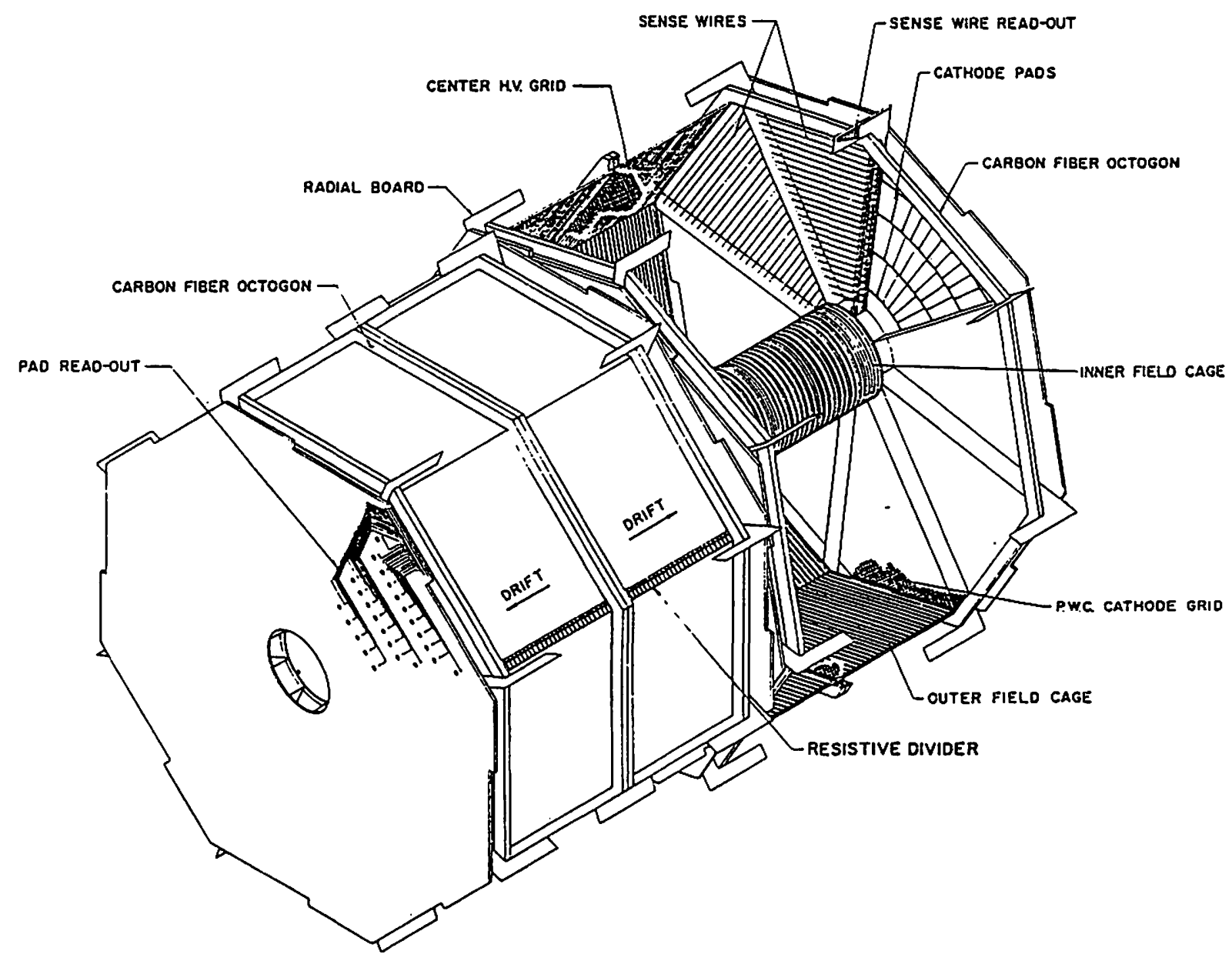

Figure 3.4: Two of the eight Vertex Time Projection Chamber modules. 


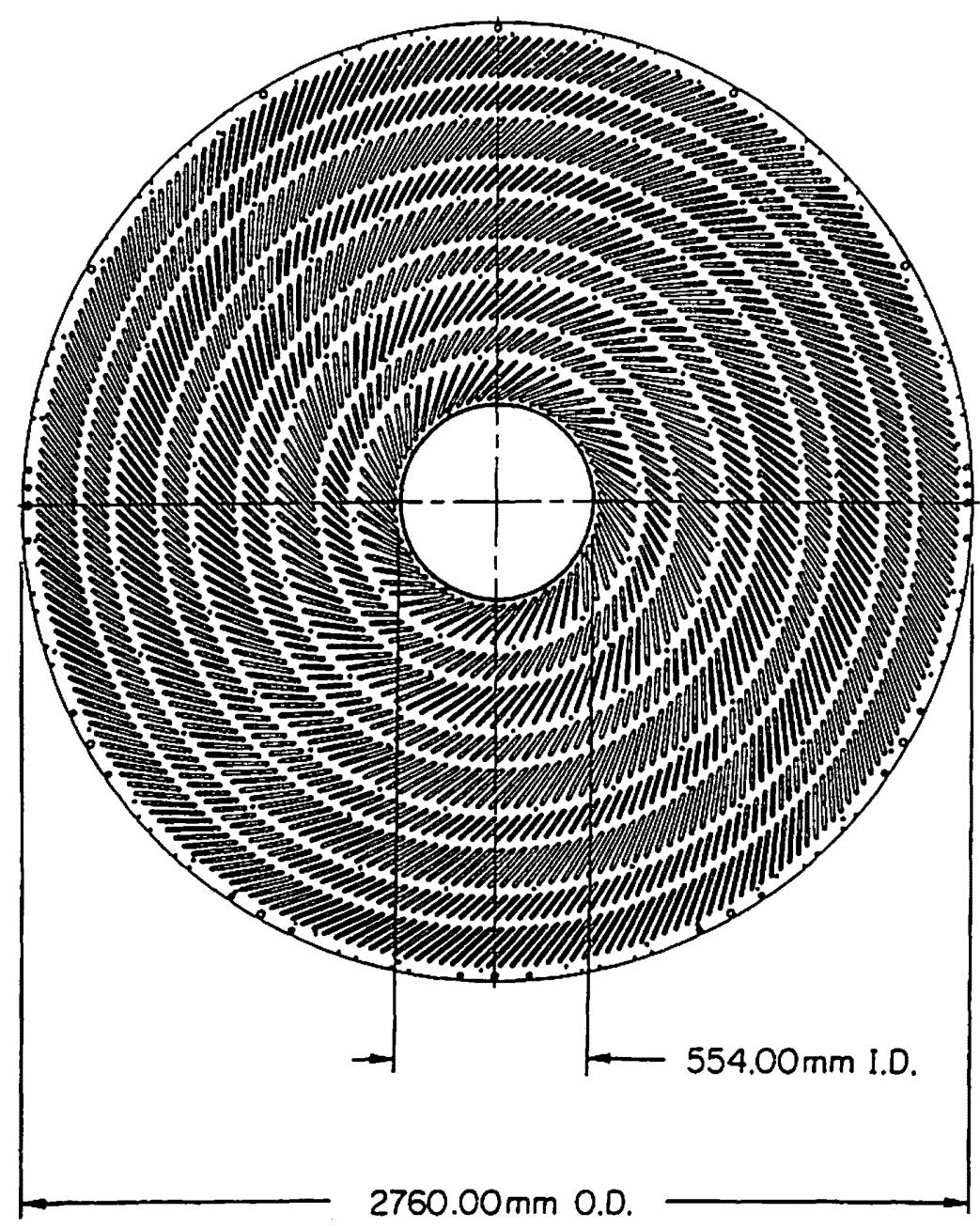

Figure 3.5: An endplate of the Central Tracking Chamber showing the arrangement of the blocks which hold the 84 layers of sense wires. 


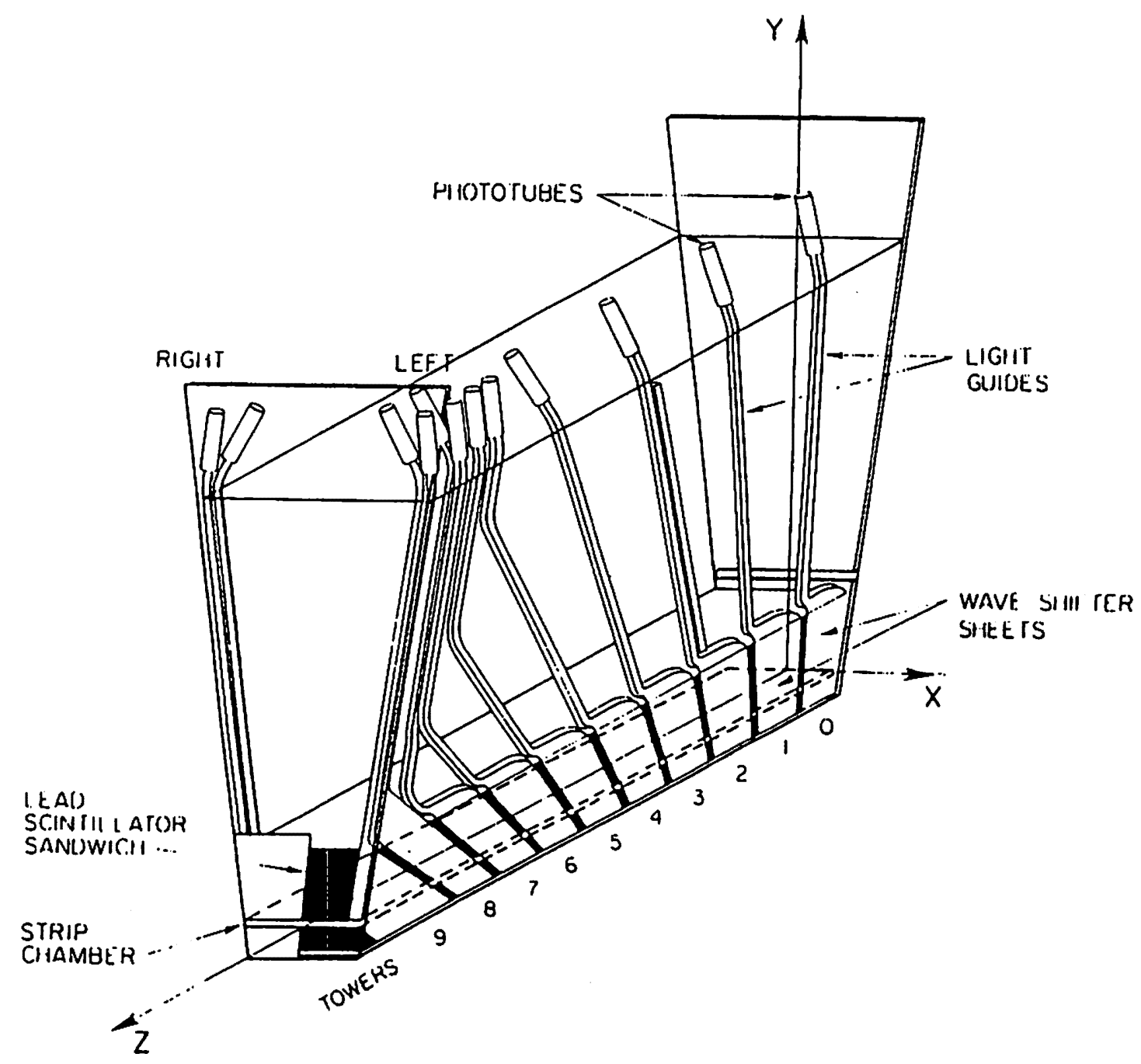

Figure 3.6: One of 48 wedges, showing the central EM calorimeter with the lightgathering system. 


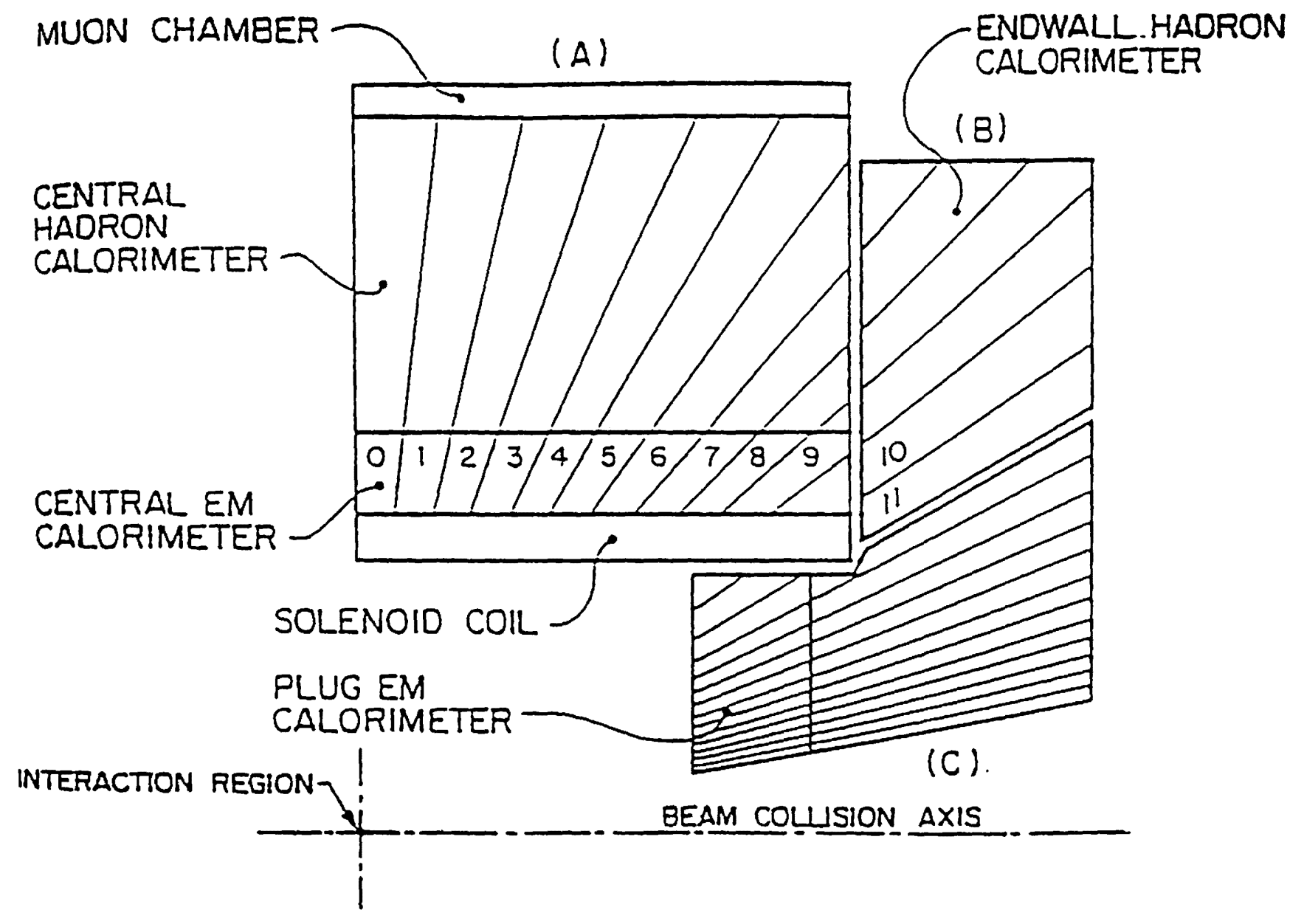

Figure 3.7: Quadrant of the calorimeter where A, B, C show central, endwall and plug, respectively. 


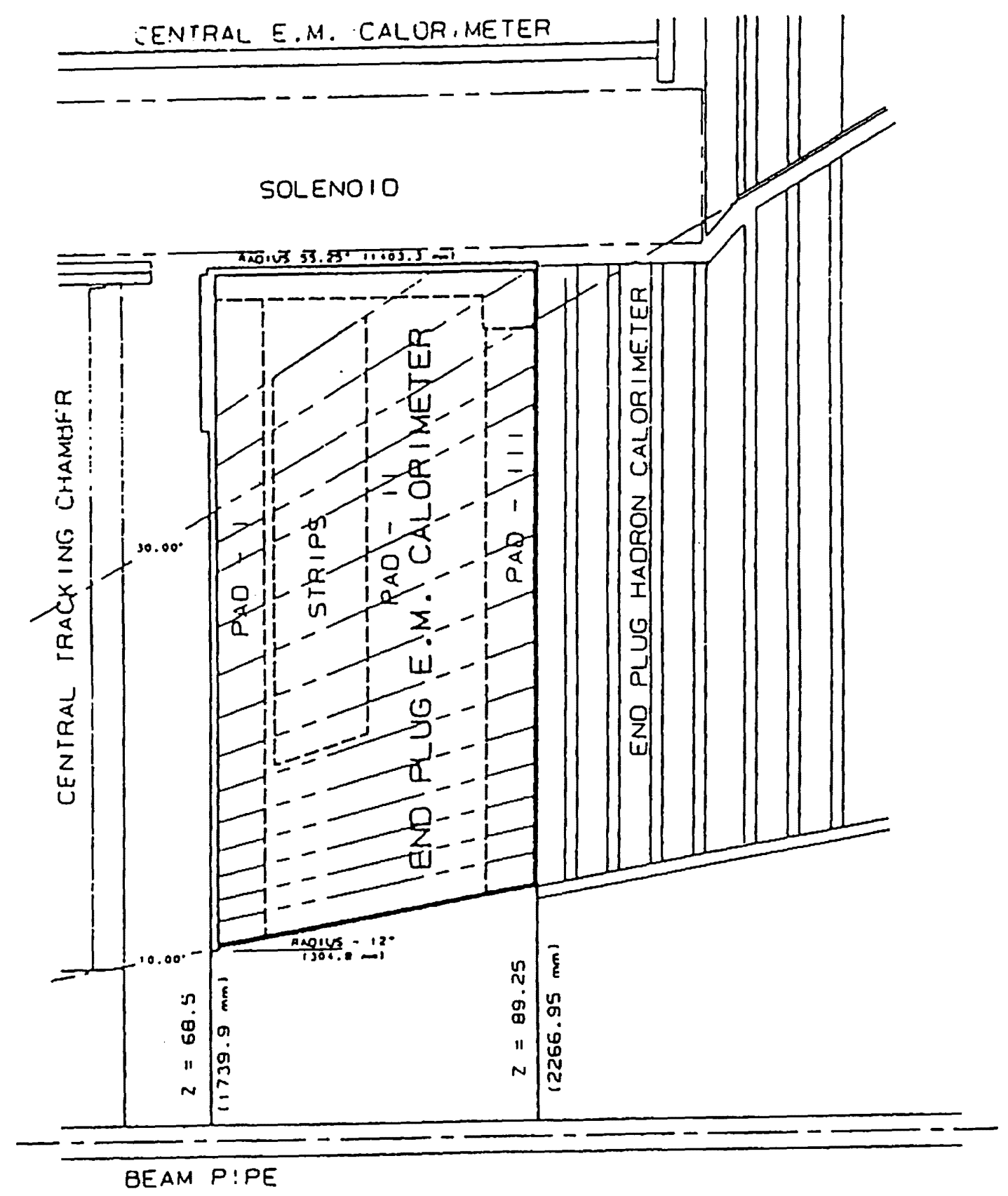

Figure 3.8: Cross sectional view of the end plug electromagnetic calorimeter. 


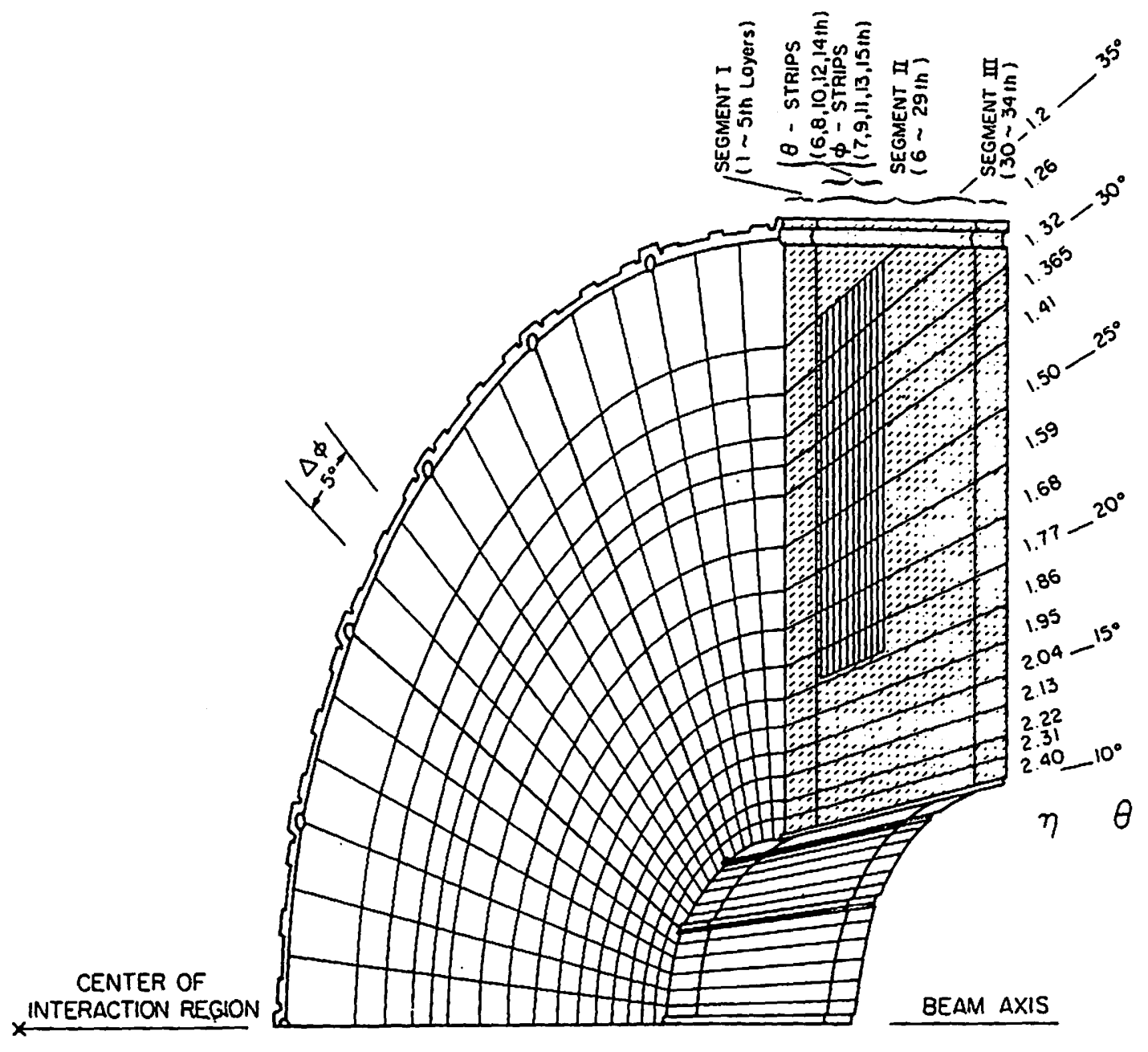

Figure 3.9: Isometric view of a PEM quadrant, showing the projective pad tower structure and the longitudinal layers. 


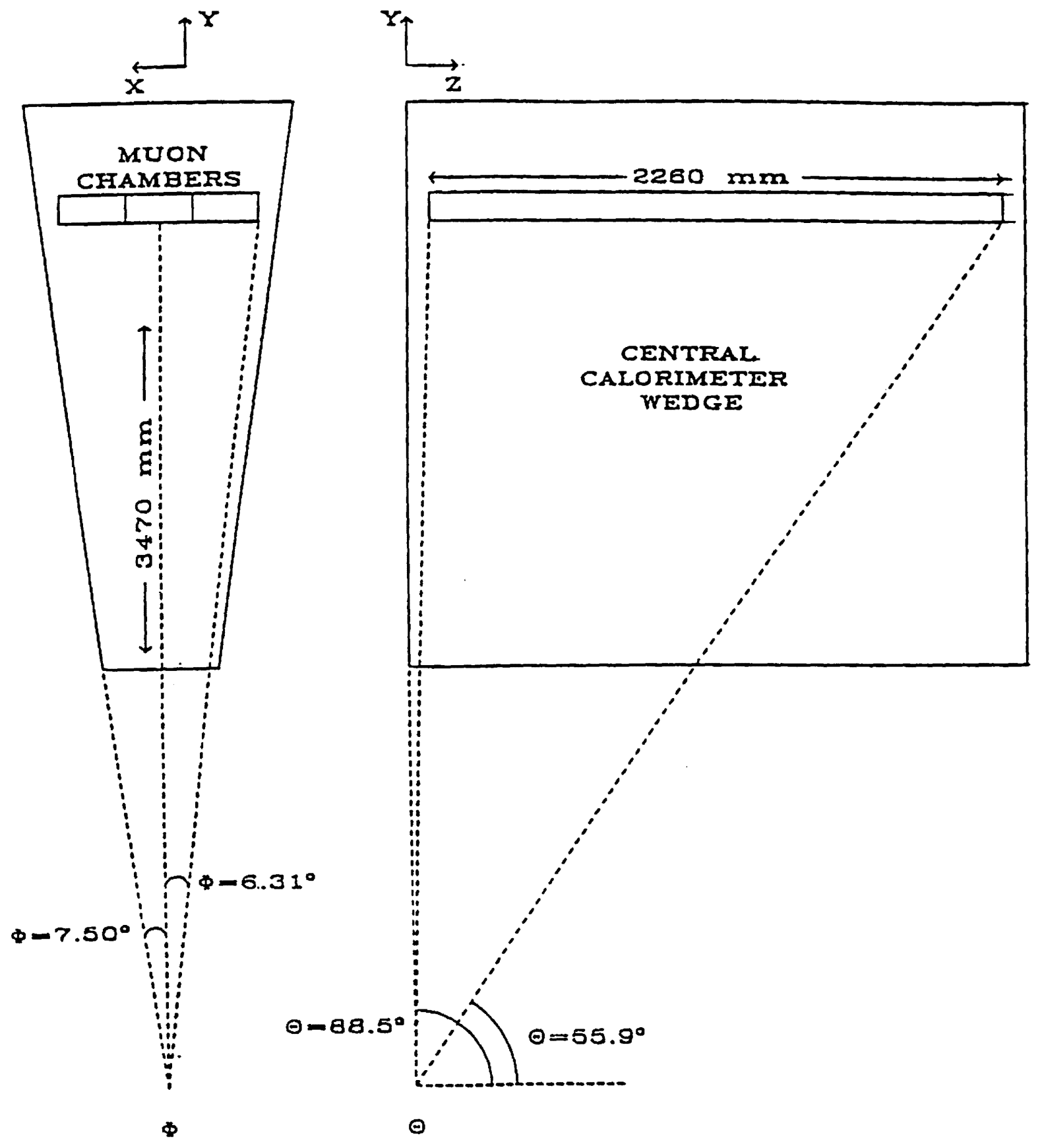

Figure 3.10: The layout of the central muon chambers in one wedge. 


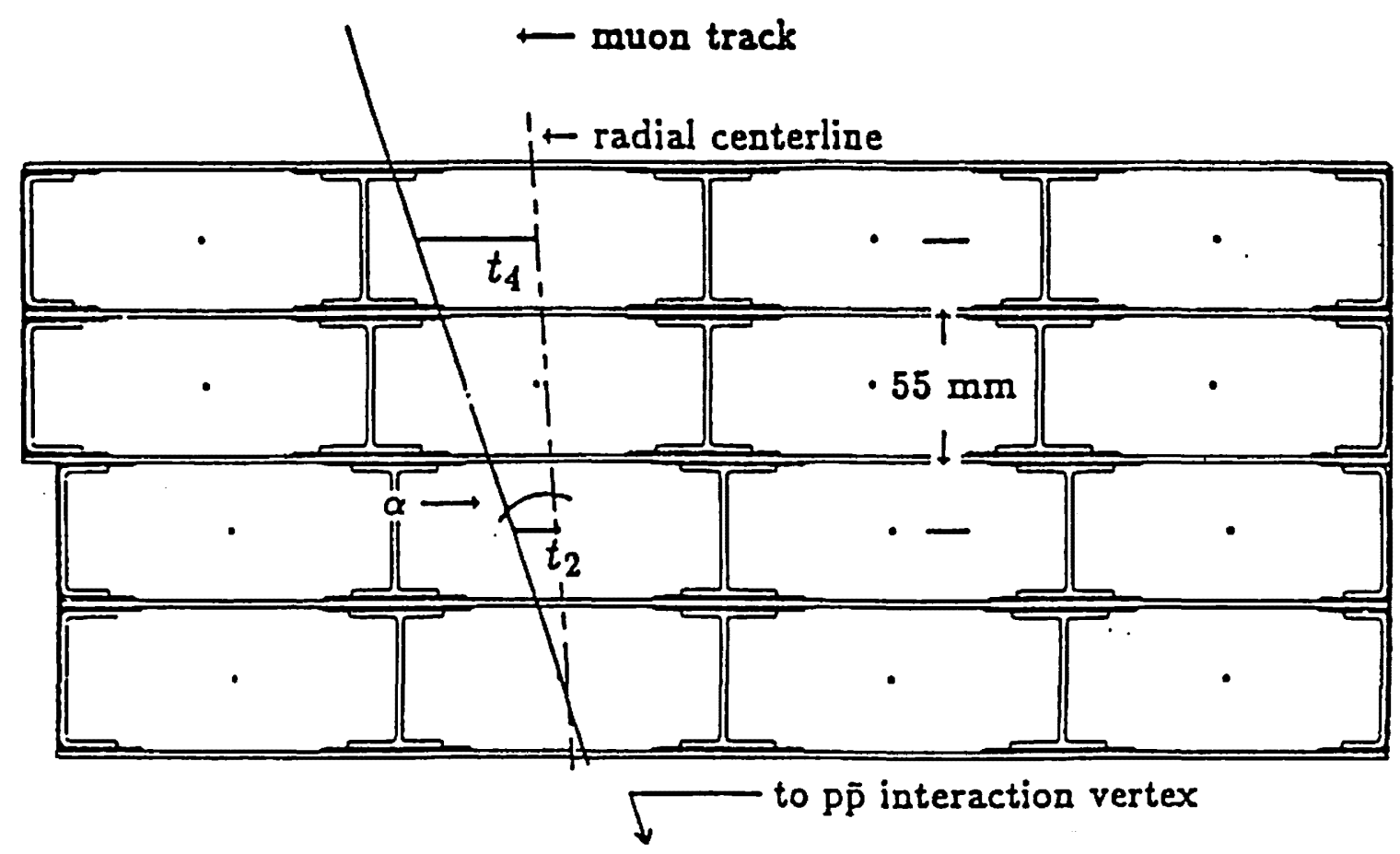

Figure 3.11: The arrangement of the four planes of the central muon chambers in a view along the beam direction $\left(\Delta \phi=4.2^{\circ}\right)$. 


\section{Chapter 4}

\section{Event Trigger and Selection}

The signature of $t \bar{t}$ events we are seeking for is the existence of two high $P_{\mathrm{T}}$ leptons (e or $\mu)$, that is, $e \mu$, ee and $\mu \mu$ events. For electrons, both the central and the plug region are considered for their detections. In this chapter, the selection for $t \bar{t}$ dilepton events in the 1988-1989 CDF data are described. The first section gives a general description about the data production scheme at CDF and about the dilepton events for this analysis. Next section describes the triggers relevant to this analysis. In $\S 4.3$ and $\S 4.4$, tools to identify electrons and muons in the CDF detector are defined and the selection cuts are described. Further cuts are applied to reject backgrounds which are expected to pass all the lepton selection cuts. This is described in $\S 4.5$. The last section describes the result when all the selection cuts are applied to the CDF data.

\subsection{Data Production}

The CDF data are controlled by the YBOS memory management system [49]. Raw data contain just $\mathrm{ADC}$ and TDC counts recorded by the various detector components of the CDF. There are standard programs which convert these unphysical data in themselves to physical information such as tower energies, tracks in the CTC, etc, and which reconstruct physical objects such as electrons, photons, muons, jets and missing $E_{\mathrm{T}}$ by 
executing clustering or other reconstruction algorithms. This stage of the data production is refered to as the "reconstruction stage" in what follows. Electrons and muons are roughly identified at this stage and registered to the corresponding electron and muon YBOS banks. These electron and muon banks contain various information for the further sophisticated identification. General users deal with these loosely identified leptons via accesses to the YBOS banks.

After the reconstruction of events, several standard programs are executed to filter events with tighter identification cuts on leptons or other physics objects and/or with the cuts based on the various physics analysis purposes such as electroweak physics, top search, etc. The CDF data production group performed data production using these standard reconstruction and filtering programs which generated several data streams. Generally users analyze these data sets according to their own analysis demands.

In order to get a better quality of the sample for the top quark search in dilepton events, further tighter lepton cuts and some event filtering criteria are imposed on the data sample from a stream containing dilepton events. The lepton identification cuts at the reconstruction stage and at the final selection stage for the top quark search will be given in $\S 4.3$ and $\S 4.4$ after the various lepton identification tools are described.

The events selected for the dilepton analysis are classified into several channels according to the regions where the leptons are detected. We use the notation CE for the central electrons and $\mathrm{PE}$ for the plug electrons. Muons detected in the central region are classified into 2 types. One is the muon detected in the central muon chamber and called the Central MUon Object (CMUO). The other is the muon identified as a minimum ionizing particle by the energy deposition in the calorimeter and called the Central Minimum lonizing Object (CMIO). In short, CMUO and CMIO are denoted by MU and $\mathrm{MI}$, respectively. According to this classification of leptons, there are 10 possible combinations of dilepton events: CEMU, CEMI, PEMU, PEMI, CECE, CEPE, PEPE, MUMU, MUMI and MIMI. However MIMI has no relevant trigger and PEPE is found to be a small contribution in the $t \bar{t}$ events ( $2 \%$ of the total dilepton acceptance). Therefore 
the 8 classes excluding MIMI and PEPE are considered in this analysis.

\subsection{Triggers}

As described before in $\S 3.2, \mathrm{CDF}$ has 4 layers of triggers $[40,50]$. The first level trigger, level 0 , required a coincidence between hits in the forward and the backward scintillation hodoscopes to select inelastic interactions of which typical rate was $\sim 50 \mathrm{kHz}$ in the 19881989 CDF run. The level 1-3 triggers reduce this high rate of $p \bar{p}$ interactions to a rate of 1-2 $\mathrm{Hz}$ at which events could be recorded on a tape. The level 1-3 triggers consist of a logical OR of several requirements which are defined to detect electrons, photons, muons, missing energy, jets and taus, and select events based on physics interests. The level-3 trigger [41] consists of a farm of 60 Fermilab Advanced Computer Program (ACP) [43] modules running the same codes as used in the CDF offline analysis. Electron and muon triggers which are relevant to this analysis are described in the following subsections.

\subsubsection{Central Electron Trigger}

The central electron trigger identifies electrons by their large EM energy deposition in the calorimeter and the existence of an associated CTC track.

\section{Level 1}

Event triggers are based on a segmentation of the calorimeter coarser than the one in the offline analysis. These trigger towers are defined as $\Delta \eta \times \Delta \phi=0.2 \times 15^{\circ}$. The central electron trigger at level 1 requires that a trigger tower have the transverse EM energy, $E_{\mathrm{T}}^{\mathrm{EM}}$, above $6 \mathrm{GeV}$ (single-tower threshold) and the sum of $E_{\mathrm{T}}^{\mathrm{EM}}$ over all the trigger towers passing the single-tower threshold be greater than $6 \mathrm{GeV}$. At this stage, $E_{\mathrm{T}}$ is calculated using the fixed event vertex position of $Z_{\text {event }}=0$.

\section{Level 2}


Electromagnetic energy clusters are formed from trigger towers by hardware processors. A clustering algorithm is that, first, a trigger tower with $E_{\mathrm{T}}^{\mathrm{EM}}>4 \mathrm{GeV}$ (a seed tower) is searched, then 4 adjacent towers are examined to be added to the seed tower if its $E_{T}^{\mathrm{EM}}$ is greater than $3.6 \mathrm{GeV}$, each attached trigger tower is then taken as a seed tower and the same procedure is repeated until no more trigger tower is found to be added. The cluster $E_{\mathrm{T}}$ is defined as a sum of $E_{\mathrm{T}}$ of all the trigger towers in a cluster. Here again $E_{\mathrm{T}}$ is calculated with $Z_{\text {event }}=0$.

CTC tracks are reconstructed in two dimension by a hardware processor called Central Fast Tracker [42]. The CFT processes fast timing information from the CTC to identify a high $P_{\mathrm{T}}$ track in the $R-\phi$ plane by comparing the CTC hits with predetermined patterns. The CFT has a momentum resolution of $\Delta P_{\mathrm{T}} / P_{\mathrm{T}}^{2} \sim 0.035(\mathrm{GeV} / \mathrm{c})^{-1}$ with a track finding efficiency of $(98 \pm 0.5) \%$. These CFT tracks are matched in azimuth with a central trigger cluster found in the calorimeter. The level-2 central electron trigger requires that a central EM trigger cluster with $E_{\Gamma}^{\mathrm{EM}}>12 \mathrm{GeV}$ exists, that the ratio of the total cluster $E_{\mathrm{T}}$ to $E_{\mathrm{T}}^{\mathrm{ENI}}$ is less than 1.125 and that there is a CFT track with $P_{\mathrm{T}}>6 \mathrm{GeV} / c$ pointing at the cluster.

\section{Level 3}

The level-3 central electron trigger [51] executes the same clustering algorithm and track finding algorithm as used in the offline analysis (see $\$ 4.3 .1$ ), except that CTC tracks are reconstructed only in 2 dimension to save the execution time. The momentum resolution of this fast track reconstruction is $\Delta P_{\Upsilon} / P_{\Gamma}^{2} \sim 0.007(\mathrm{GeV} / \mathrm{c})^{-1}$. The level-3 central electron trigger requires the same criteria as in the level-2 central electron trigger, $E_{\mathrm{T}}^{\mathrm{EM}}>12 \mathrm{GeV}, E_{\mathrm{T}} / E_{\mathrm{T}}^{\mathrm{EM}}<1.125$, a CTC track with $P_{\mathrm{T}}>6 \mathrm{GeV} / c$ pointing at the EM cluster. Furthermore, a cut on the lateral shower shape of the EM cluster measured with the calorimeter towers, $L_{\text {shr }}<0.5$, is required if $E_{\mathrm{T}}^{\mathrm{EN}}<20 \mathrm{GeV}$, where $L_{\text {shr }}$ is defined by Equation (4.6). The transverse energy calculation uses a measured event vertex. 


\subsubsection{Plug Electron Trigger}

\section{Level 1}

The calorimeter segmentation used in the plug electron trigger is the same as the one in the central region. A similar requirement is imposed while the single-tower threshold is slightly lowered from $6 \mathrm{GeV}$ to $4 \mathrm{GeV}$ in order to account for the physically smaller trigger tower size in the plug compared to that in the central. The total EM $E_{\mathrm{T}}$ summed over all the trigger towers with $E_{\mathrm{T}}$ above a signle-tower threshold must be greater than $6 \mathrm{GeV}$.

\section{Level 2}

A similar clustering algorithm as used for the central electrons is performed. It requires that $E_{\mathrm{T}}^{\mathrm{EM}}>23 \mathrm{GeV}$ and that the ratio of the total cluster $E_{\mathrm{T}}$ to $E_{\mathrm{T}}^{\mathrm{EN}}$ be less than 1.125. No requirement on tracks is imposed since the CTC performance is limited in this $\eta$ region.

\section{Level 3}

The level-3 trigger executes the same clustering algorithm as used in the offline analysis. No effective filtering was performed at the level-3 plug electron trigger (the threshold of $E_{\mathrm{T}}^{\mathrm{EM}}$ was $7.5 \mathrm{GeV}$ ).

\subsubsection{Central Muon Trigger}

\section{Level 1}

The central muon level-1 trigger [52] uses information exclusively from the muon chamber TDC's. The timing information is used to determine the momentum of a charged track detected in the muon chamber, as described in $\$ 3.1 .4$. The level-1 muon trigger requires that the time difference of hits in alternate two muon chambers be lower than a programmable threshold, thereby imposing a minimum $P_{\mathrm{T}}$ cut on the charged particles. A cut of $P_{\mathrm{r}}>5 \mathrm{GeV} / c$ was used during approximately the first third of the run and a 


\begin{tabular}{|l|l|l|}
\hline Central electron & $12 \mathrm{GeV}$ & CEMU,CEMI,CECE,CEPE \\
\hline Central muon & $9 \mathrm{GeV} / c$ & CEMU,CEMI,MUMU,MUMI,PEMU \\
\hline Gas photon & $23 \mathrm{GeV}$ & PEMI \\
\hline
\end{tabular}

Table 4.1: Triggers and corresponding dilepton event types which are selected by the trigger.

3-GeV/c threshold later on.

\section{Level 2}

The level-2 trigger requires the existence of a CFT track with $P_{\mathrm{T}}>9.2 \mathrm{GeV} / c$. This track is propagated to a muon chamber region and required to match with a track segment recorded at the muon chambers within $15^{\circ}$ in azimuth.

\section{Level 3}

At the level 3, we reconstruct a CTC track using the same code as used in the offline analysis but only in 2 dimension to save the reconstruction time, and impose a momentum cut of $P_{\mathrm{T}}>11 \mathrm{GeV} / c$ and a position match better than $\pm 10 \mathrm{~cm}$ in the azimuthal direction with a track segment in the muon chamber.

\subsubsection{Triggers for the Dilepton Events}

Each dilepton channel is selected by one or more lepton triggers. The relations between the channels and the triggers by which events are recorded are shown in Table 4.1.

\subsection{Electron Selection}

Electron identification in CDF [53] is described in this section. Other descriptions on the electron identification which are not exactly same but similar to the one described here can also be found in earlier publications $[20,54]$. 
High $P_{\mathrm{T}}$ electrons are identified basically by the large energy deposition in the EM calorimeter and the existence of an associated track. However there are backgrounds which fake an electron signature in the detector. One of them is a charged pion interacting in the EM calorimeter. Most of charged $\pi$ 's are discriminated by the energy deposition in the hadronic calorimeter than that in the EM calorimeter. But some fraction of charged $\pi$ 's interact and deposit a large part of their energy in the EM calorimeter. Since a charged $\pi$ leaves an associated track in the tracking chamber, it looks like an electron. Another source of fake electron backgrounds is $\pi^{0} / \gamma^{\prime}$ s with an overlapped charged track. Both $\pi^{0}$ and $\gamma$ make an energy deposition in the EM calorimeter. In the case that a charged $\pi$ passes near the $\pi^{0} / \gamma$ in the EM calorimeter, its track looks associated to that EM energy deposition. This also mimics an electron signature. On top of these fake electron backgrounds, there are electrons which are not related to hard $p \bar{p}$ collisions, such as electrons from photon conversions $(\gamma \rightarrow e e)$ and Dalitz decays $\left(\pi^{0} \rightarrow e^{+} e^{-} \gamma\right)$. In order to reject these fake and real electron backgrounds, several identification tools are developed which are described in this section.

\subsubsection{Central Electron}

\section{Clustering}

A segmentation of the central calorimeter at the offline analysis level is the same as the physical one of the detector. The $\eta$ width is 0.11 and the $\phi$ width is $15^{\circ}$. The clustering algorithm starts from searching a seed tower which has $E_{\mathrm{T}}^{\mathrm{EN}}>3 \mathrm{GeV}$. The transverse energy $E_{\mathrm{T}}$ is calculated using the polar angle $\theta$ connecting the measured event vertex $Z_{\text {event }}$ and the center of a tower. The tower center is defined as the middle of the tower width in terms of the polar angle and the azimuthal angle at the depth of the shower maximum $\left(\sim 5.9 X_{0}\right)$.

Then adjacent two towers in $\eta$ direction are examined and added if $E_{\mathrm{T}}^{\mathrm{NN}}>0.1 \mathrm{GeV}$. In $\phi$ direction, we do not add towers since EM showers are well contained in one tower in this direction. The maximum number of towers in a cluster is 3 but in most cases an 
EM cluster contains only one or two towers since the EM shower size is typically $2 \mathrm{~cm}$ in width and is smaller than a tower size of $R \Delta \phi \times \Delta Z \sim 45 \mathrm{~cm} \times 24 \mathrm{~cm}$. The energy $E$ of a cluster is calculated by summing the tower energies in a cluster.

After the clustering, three successive corrections on the EM energy part are provided in the offline analysis [55]. The first one is based on a response map in a single tower from test beam electron data. This correction is done according to the shower center's location in a tower which is measured with the CES (The shower position measurement with the CES is described later in this section). The second correction is for towerto-tower response variations. This correction is obtained by examining tower-to-tower differences in the ratio of the cluster energy to the momentum of a track associated to the cluster $(E / P)$ using real data. This correction sets $E / P$ of all the towers to 1 in average. However, an electron can emit a photon which is contained in a same EM cluster while the electron looses its momentum due to this radiation. Therefore the actual value of $E / P$ tends to be greater than 1 . One thus needs an overall scale which takes the radiation into account. This final correction is determined to be 1.026 by the $E / P$ distribution in the range of $E / P<1.4$ for the $W$ Monte Carlo simulation incorporating photon radiation [56]. At the reconstruction stage, none of these corrections are applied.

The total $E_{\mathrm{T}}$ of a cluster is defined as $E \sin \theta$, where $E$ is the cluster energy and $\theta$ is the polar angle of the cluster centroid with respect to the measured event vertex. The cluster centroid is calculated as the energy weighted mean of the tower centers in a cluster. $E_{\mathrm{T}}^{\mathrm{EMI}}$ and $E_{\mathrm{T}}^{\mathrm{HAD}}$ are defined similarly. An EM cluster is pre-required to satisfy $E_{\mathrm{T}}^{\mathrm{EM}}>5 \mathrm{GeV}$ and $H A D / E M<0.125$ at the first level of the identification, where $H A D / E M$ is the ratio of the hadronic energy to the EM energy.

\section{Shower Detection in the CES}

The lateral shape of an electromagnetic shower is measured with the Central Electromagnetic Strip chamber (CES) which is located approximately at the shower maximum depth $\left(\sim 5.9 X_{0}\right)$ in the central electromagnetic calorimeter. The clustering algorithm 
starts from searching seed channels which must have energy greater than $>0.5 \mathrm{GeV}$. The highest energy channel is picked up and channels in a fixed window centered at the seed channel are attached. The number of channels in a window was 11 including a seed tower. Next, the highest seed channel is selected from those which are outside the first cluster and the only channels which were not used in the previous clustering are attached within a new window. The same procedure is repeated until no seed channels are found.

\section{Track Associated with an EM Cluster}

CTC tracks are reconstructed in 3 dimension from $R-\phi$ measurments by the axial superlayers and $R-z$ measurements by the streo superlayers with the momentum resolution of $\Delta P_{\mathrm{T}} / P_{\mathrm{T}}^{2} \simeq 0.002(\mathrm{GeV} / c)^{-1}$. The highest $P_{\mathrm{r}}$ track in those pointing to an EM cluster is picked up as the one which is associated with an EM cluster.

\section{Definition of the Fiducial Region}

In order to ensure a correct energy measurement, a fiducial region is defined so as to avoid inactive detector region. For central electrons, the fiducial region is given by a set of requirements:

- Seed tower of a cluster must have a tower number of $0-8$. Tower 9 does not have enough depth coverage for the EM calorimeter.

- A track associated with an EM cluster is propagated to the CES, $R=184 \mathrm{~cm}$. The track must be at least $2.5 \mathrm{~cm}$ away from a $\phi$ boundary of the wedge.

- An absolute value of $Z$ coordinate of a track propagated to the CES must be $>9$ $\mathrm{cm}$. This is meant to avoid a crack at $\theta=90^{\circ}$. 


\section{Identification Variables}

In order to improve a quality of electron candidates, several variables are defined and used to reject backgrounds. The main sources of background are mentioned at the top of this section.

\section{(1) $H A D / E M$}

A ratio of the hadronic energy to the electromagnetic energy. This quantity is used to discriminate charged $\pi$ 's which tend to have more energy deposited in the hadronic calorimeter than electrons. Figure 4.3 shows the $H A D /(H A D+E M)$ distributions for test beam electrons, for test beam pions, and for central electrons from $W \rightarrow e \nu$ events.

\section{(2) $E / P$}

A ratio of $E_{\mathrm{T}}^{\mathrm{EM}}$ to P.r. This ratio is close to 1 for electrons. One of the sources which mimic the electron signature in the detector is $\pi^{0} / \gamma$ 's with a charged hadron which happens to pass near the $\pi^{\%} / \gamma$ 's. This overlap of a charged hadron looks an EM cluster with an associated track. However, the $E / P$ ratio is not necessarily 1 in this case due to its accidental characteristics, and $E / P$ is useful to reject this type of backgrounds. Figure 4.4 shows the $E / P$ distributions for the central electrons from the $Z \rightarrow e e$ events and for the EM clusters in a jet sample. The jet sample consists of the events triggered by the pre-scaled jet trigger with an $E_{\text {T }}$ threshold of $20 \mathrm{GeV}$. Since this sample is dominated by the ordinary QCD jet events, most of the EM clusters in the sample can be considered as fake electrons.

\section{(3) Lateral shower shape: $\chi_{\text {strip }}^{2}$}

Comparing a shower shape measured in the CES with the one obtained from test beam electrons, one can check the consistency of the measured shower shape with the expected electron shower shape [57]. The variable $\chi_{\text {strip }}^{2}$ defined by Equation (4.3) uses a shower shape in strip view (along the beam direction). We minimize the following function 
varying two parameters, $Z$, the $Z$ position of the center of a shower, and $E$, the electron energy.

$$
\chi^{2}(Z, E)=\sum_{i=1}^{n} \frac{\left(E_{i}^{\text {meas }}-E q_{i}^{\text {pred }}(Z)\right)^{2}}{\sigma_{i}^{2}(Z)}
$$

where $E_{i}^{\text {meas }}$ is the measured energy in the channel $i, q_{i}^{\text {pred }}$ is the predicted fraction of the total energy in the channel $i$ which is obtained from $50-\mathrm{GeV} / c$ test beam electrons, and $\sigma_{i}$ denotes the energy fluctuation of a single-channel response. In the summation, 11 channels are examined $(n=11)$ corresponding to about $15 \mathrm{~cm}$ which is sufficient to contain a full electron shower. The response fluctuation $\sigma_{i}$ for each channel is parameterized as

$$
\sigma_{i}^{2}(z)=(0.026)^{2}+(0.096)^{2} q_{i}^{\text {pred }}(z)
$$

The parameterization is based on $10-\mathrm{GeV} / c$ electron test beam data.

Using the shower center position obtained above, $\chi_{\text {strip }}^{2}$ is defined as

$$
\chi_{\text {strip }}^{2}=\frac{1}{4}\left(\frac{E_{\mathrm{CENI}}}{10}\right)^{0.747} \sum_{i=1}^{n} \frac{\left(q_{i}^{\text {meas }}-q_{i}^{\text {pred }}\left(Z_{C E S}\right)\right)^{2}}{\sigma_{i}^{2}\left(Z_{C E S}\right)}
$$

where $E_{\mathrm{CEM}}$ is the energy measured by the central EM calorimeter, $q_{i}^{\text {meas }}$ is the measured fractional energy in the channel $i$. The energy obtained by minimizing the function (4.1) is not used because the energy resolution of the strip chamber measurement is worse ( $20 \%$ and $30 \%$ for $50 \mathrm{GeV} / c$ and $10 \mathrm{GeV} / c$ electrons, respectively) than the one measured by the CEM. An energy dependent factor of $E^{0.7 \cdot 17}$ is introduced to compensate for the energy dependence of the $\chi_{\text {strip }}^{2}$, which comes from the energy fluctuation of a singlechannel response. The fluctuation is dependent on the number of secondary electrons traversing the CES plane. Since the number of secondary electrons depends on the energy of a primary electron $(N \propto E)$, the fluctuation is dependent on the primary electron energy. The parameterization is determined from test beam electrons of various energies from $10 \mathrm{GeV}$ to $200 \mathrm{GeV}$. Figure 4.1 shows the distributions of $\chi_{\text {strip }}^{2}$ for electron candidates in the $Z \rightarrow e e$ events and for EM clusters in the jet events. 
In the same way, the shower shape parameter in $z$ direction, $\chi_{\text {wire }}^{2}$, is defined using a wire view of the EM shower. But it is not used in this analysis because the position resolution of the CES for $R-\phi$ measurements is worse than that for $z$ measurements (see §3.1.3).

The $\chi_{\text {strip }}^{2}$ is useful to discriminate against an EM cluster in which more than one particle are contained. Photons from $\pi^{0} \rightarrow \gamma \gamma$ decay have a minimum opening distance $d(\mathrm{~cm})$ at a radius $R(\mathrm{~cm})$ from the beam line that is related to the $\pi^{0} P_{\mathrm{T}}$ as

$$
d(\mathrm{~cm})=\frac{2 R M_{\pi^{0}}}{P_{\mathrm{T}}}
$$

where $M_{\pi^{0}}=0.135 \mathrm{GeV} / c^{2}$ is $\pi^{0}$ mass, $P_{\mathrm{T}}$ is in $\mathrm{GeV} / c$. At the strip chamber radius, $R=184 \mathrm{~cm}$, we have $d \simeq 50 / P_{\Upsilon} \mathrm{cm}$. Since the window size of the $\chi_{\text {strip }}^{2}$ is $\sim 15 \mathrm{~cm}, \gamma$ 's with $d \lesssim 7.5 \mathrm{~cm}$ or $\gamma$ 's from $\pi^{0}$ of $P_{\text {r }}$ above $\sim 7 \mathrm{GeV} / c$ will be contained in a same strip cluster. The resulting $\chi_{\text {strip }}^{2}$ will have a large value due to the existence of an additional shower peak in a cluster. A typical CES shower has $~ 99 \%$ of the energy contained in $\pm 2.5 \mathrm{~cm}$ around the shower center. Therefore the presence of two photons will be identified by a large value of $\chi_{\text {strip }}^{2}$ up to $P_{1}^{\pi^{0}} \sim 20 \mathrm{GeV} / c(d \simeq 2.5 \mathrm{~cm})$. Above this momentum, the two photons get closer to make it difficult to recognize two showers in the CES.

This variable is also used to discriminate against charged pions which interact and deposite the energy in the CEM. They have usually broader shower shapes than that for electrons. Figure 4.2 shows the distribution of the average strip chamber $\chi^{2}=$ $\left(\chi_{\text {strip }}^{2}+\chi_{\text {wirc }}^{2}\right) / 2$, for the test beam electrons, charged pions and $W$ electrons. The strip $\chi^{2}$ is fully made use of in the photon cross section measurement at CDF.

(4) Position matching: $D_{X}$ and $D_{\%}$

The position difference in $R-\phi$ view between the position measured in the CES and that 
of the track extrapolated to the CES radius, $D_{X}$, is defined as

$$
D_{x}=R \Delta \phi \quad(R=184 \mathrm{~cm}) .
$$

The CES position is obtained by fitting the shower shape in wire view to a predicted shape in a similar way as in the $\chi_{\text {strip }}^{2}$ calculation.

The position difference along the beam direction $D_{Z}$ is calculated from a position measurment in the CES and the extrapolated track position at the CES radius.

Figure 4.5 shows the distributions of $D_{X}$ and $D_{Z}$ for electrons in the $Z \rightarrow e e$ events and for EM clusters in the jet sample.

This matching requirement is useful in rejecting overlaps of $\pi^{0} / \gamma^{\prime}$ s and $\pi^{ \pm}$where an accidentally associated track of charged $\pi$ is expected to have a worse position matching than that of an electron.

(5) Lateral shower sharing: $L_{\text {shr }}$

This variable $[20,54]$ describes a lateral sharing of the EM shower energy among the towers in a cluster:

$$
L_{\mathrm{shr}}=0.14 \sum_{i} \frac{E_{i}^{\mathrm{rncas}}-E_{i}^{\mathrm{pred}}}{\sqrt{(\Delta E)^{2}+\left(\Delta E_{i}^{\mathrm{pred}}\right)^{2}}}
$$

where $E_{i}^{\text {meas }}$ is the energy deposited in the tower $i, E_{i}^{\text {pred }}$ is the energy expected in the tower $i, \Delta E$ is the uncertainty in the energy measurement with the CEM ( $\Delta E=$ $0.14 \sqrt{E}$ ), and $\Delta E_{i}^{\text {pred }}$ is the error associated with $E_{i}^{\text {pred }}$. The sum runs over the two towers in $\eta$ direction adjacent to the seed tower. The expected tower energy $E_{i}^{\text {pred }}$ was determined from test beam electron data [51] as a function of the seed tower energy and the direction of the CES shower center relative to the event vertex. The error $\Delta E_{i}^{\text {pred }}$ was determined by propagating an error of the shower center measurement in the CES to the predicted energy $E_{i}^{\text {pred }}$. Figure 4.6 shows $L_{\text {shr }}$ distributions for the electrons in the $Z \rightarrow e e$ events and for the EM clusters in the jet sample. 


\section{Conversion Removal}

Electrons from photon conversions $\left(\gamma \rightarrow e^{+} e^{-}\right)$and Dalitz decays $\left(\pi^{0} \rightarrow e^{+} e^{-} \gamma\right)$ have to be removed from the electron sample. Both are called "conversion" electrons here. These conversion electrons can be identified with a high efficiency of $(88 \pm 4) \%$ in the CDF $[20,58]$. A photon conversion can occur before entering the VTPC, in the beam pipe or in the inner wall of the VTPC. This type of photon conversions is called inner photon conversion. Also a photon can convert after exiting the VTPC, in the outer wall of the VTPC or in the inner wall of the CTC. This is called outer photon conversion. In both cases, the electron track is detected in the CTC, but the outer photon conversions do not leave tracks in the VTPC.

The ratio of the number of VTPC wire hits to the number of wires which are supposed to be traversed by the particle, VTPC hit occupancy $\left(f_{\mathrm{VTPC}}\right)$, can be used to discriminate charged particles from neutral particles in the VTPC. The electrons from outer photon conversions have a low value of $f_{V T P C}$. In order to remove electrons from inner photon conversions and Dalitz decays, an additional oppositely charged CTC track is searched and the invariant mass, $m_{r c c}$, is examined. Two electrons from these processes are expected to form a low mass. An electron which fails one of the following requirements is removed as a "conversion" electron,

- $f_{\mathrm{VTPC}}>0.2$

- $m_{e c}>0.5 \mathrm{GeV}$.

Figure 4.7 shows a distribution of conversion points for conversion electron sample in terms of the radial distance, $R$, from the beam line. One sees clear peaks around the VTPC inner wall $R \sim 2.5 \mathrm{~cm}$ and the CTC inner wall $R \sim 28 \mathrm{~cm}$.

\section{Isolation}

After a heavy quark is produced, it fragments or hadronizes into a heavy hadron containing the heavy quark and some lighter hadrons. Succesively the heavy hadron decays 
into several stable particles. As discussed in $\$ 2.5$, the leptons from a top decay are typically well separated from other particle activities. Also due to the large mass of the top quark, additional particles $(X)$ produced in the top quark fragmentation, $t \rightarrow t$-meson $+X$, are expected to carry only a small fraction of the initial parent quark's momentum (hard fragmentation). Given these heavy quark fragmentation and decay properties, leptons from top decays are expected to be more isolated than those from lighter bottom or charm quark decays. Therefore, an isolation variable which describes how well an electron is isolated is useful to get a better signal-to-background ratio. The isolation cut is also useful to reject fake electron backgrounds from particle misidentification, and the conversion electrons, since these background particles originate from ordinary $Q C D$ jets and are usually surrounded by other particle activities from the jets. Additional energy near the lepton can also originate from the hadronization of gluons radiated by the initial and final state partons (gluon radiation), or from the spectator partons in $p \bar{p}$ interactions (underlying event).

The isolation variable is defined as

$$
E_{\mathrm{T}}^{\mathrm{conc}}=E_{\mathrm{T}}(R=0.4)-E_{\mathrm{T}}^{\mathrm{EM}}(e)-E_{\mathrm{T}}^{\mathrm{HAD}}(e)
$$

where $E_{\mathrm{T}}(R=0.4)$ is the total $E_{\mathrm{T}}$ of towers within radius 0.4 in $\eta$ - $\phi$ space, $E_{\mathrm{T}}^{\mathrm{EM}}(e)$ and $E_{\mathrm{T}}^{\mathrm{HAD}}(e)$ are the transverse electromagnetic energy and the transverse hadronic energy of an electron cluster. The $E_{\mathrm{T}}^{\mathrm{cone}}$ measures an extra activity around the electron. Figure 4.8 shows an isolation distribution for the electrons from $t \bar{t}$ Monte Carlo events with $M_{\text {Lop }}=90 \mathrm{GeV} / c^{2}$ by the ISAJET program [59], calculated with the CDF detector simulation program, CDFSIM [60].

\section{Definition of the Central Electron Selection}

The requirements on the central electrons in the top quark search are:

1. Fiducial cuts 
2. $E_{\mathrm{T}}^{\mathrm{EM}}>15 \mathrm{GeV}$ and $P_{\mathrm{T}}>10 \mathrm{GeV} / c$

3. $E_{\mathrm{T}}^{\text {cone }}<5 \mathrm{GeV}$

4. Identification cuts

(Tight cuts)

- $H A D / E M<0.05$

$-L_{\mathrm{shr}}<0.2$

- $E / P<2.0$

- $D_{X}<1.5 \mathrm{~cm}$ and $D_{Z}<3.0 \mathrm{~cm}$

$-\chi_{\text {strip }}^{2}<10.0$

(Loose cut)

- $H A D / E M<0.055+0.045 \times E / 100$

5. Conversion removal.

The $E_{\mathrm{T}}$ cut at $15 \mathrm{GeV}$ is chosen so that most of the electrons from $b \bar{b}$ process or the misidentified electrons are removed while the large portion of electrons from top events is preserved. These backgrounds have lepton $P_{T}$ spectra that fall much faster than the electrons of $t \bar{t}$ events. The predicted number of $e \mu$ events with the transverse momentum of both leptons above a common threshold $P_{\mathrm{T}}^{\min }$ is shown in Figure 4.9 [19]. The significant difference between the signal and background lepton spectra motivates a choice of $P_{\mathrm{T}}$ threshold of $15 \mathrm{GeV} / c$. Thus events in the signal region are required to have both leptons with $P_{\mathrm{T}}$ above $15 \mathrm{GeV} / c$. The trigger efficiency is high (approximately $98 \%)$ at this threshold.

Since the clustering algorithms are different in the level-2 trigger and in the offline analysis, and also since the definition of $E_{\text {个 }}$ is different due to the different vertex position used, the offline $E_{\mathrm{T}}$ and trigger $E_{\mathrm{T}}$ have different values. Therefore one has to estimate the trigger efficiency as a function of the offline $E_{\mathrm{T}}$. The offline $E_{\mathrm{T}}$ threshold of $15 \mathrm{GeV}$ turned out to be quite efficient for the $12-\mathrm{GeV}$ trigger- $E_{\mathrm{C}}$ threshold. The details are described in $§ 5.1 .3$. 
The loose cut is applied to one of the two electrons of the central dielectron events. The central electron identification in the CDF has high efficiency, keeping the largest rejection power against fake electron backgrounds compared to other lepton identifications, such as plug electron and muon identification. The plug electron identification suffers from the limited ability of track momentum measurement in the plug region. The central muon identification has a disadvantage from hadron punch-through background due to rather thin absorber thickness of the central calorimeter. Since the central electron identification shows the best performance, we do not have to require tight identification cuts on both central electrons. The energy dependent $H A D / E M$ cut accounts for the shower leakage of the electron energy which was determined from test beam data.

\subsubsection{Plug Electron}

\section{Clustering}

The calorimeter segmentation in the offline analysis is taken to be $\Delta \eta \times \Delta \phi=0.1 \times 5^{\circ}$. This software segmentation in $\eta$ is different from the physical one (see §3.1.3). The clustering algorithm is essentially same as the one for central electrons but the number of towers examined with respect to a seed tower is now $\Delta \eta \times \Delta \phi=5 \times 5$.

The energy $E$ is a sum of the tower energies in a cluster. The calculation of $E_{\mathrm{T}}$ is same as the one for the central. The energy correction for plug electrons consists:

- Dead layer correction

- Non-linearity correction

- Tower map correction

- Quadrant gain correction.

The PEM had several dead layers from the beginning of the run. The dead layer correction corrects for this effect. For historical reasons, the dead layer correction used in the reconstruction stage was based on the longitudinal shower profile of $100 \mathrm{GeV} / c$ test 
beam pions. A recorrection of the energy is possible by calculating the predicted energy in the dead layers using electron shower profile parametrized from test beam electrons The magnitude of the dead layer correction is at most $20 \%$. The non-linearity correction accounts for the effects of the longitudinal shower leakage and the gain saturation of the chambers. Using test beam electron data, the correct energy is fitted to a quadratic form as a function of the measured energy. The correction is a few $\%$ for the electron energy around $50 \mathrm{GeV}$. The tower map correction corrects for tower-to-tower variations of the calorimeter response. The correction factor (order of $\sim 5 \%$ ) for each tower was determined as a relative ratio to a reference tower in a reference quadrant by injecting $100-\mathrm{GeV}$ test beam electrons into the center of each tower. But the correction factors for the towers in a single quadrant were normalized so that all the quadrant have a same response in average as that for the reference quadrant. In order to account for the quadrant-to-quadrant response variations, the quadrant gain corrections relative to the reference quadrant were determined by looking at the $Z$ mass and the electron $E_{\mathrm{T}}$ distribution in $W \rightarrow e \nu$ events. The correction was found to be at most $15 \%$. At the reconstruction stage, the dead layer correction based on pion test beam data and the quadrant gain corrections were applied. The dead layer recorrection, the non-linearity correction and the tower map correction have to be performed in furhter analysis by each user.

Plug electrons are roughly identified at the reconstruction stage of the data production. The requirments are that a plug EM cluster must satisfy $E_{\mathrm{T}}^{\mathrm{EN}}>5 \mathrm{GeV}$ and $H A D / E M<0.125$.

\section{Definition of the Fiducial Region}

To ensure the correct energy measurement, the fiducial region is defined:

- A seed tower must not be in outer 2 pads nor inner 2 pads. This region has not enough depth coverage. This requirement is approximately equivalent to the cut of $1.26<|\eta|<2.22$ on the cluster centroid. 
- A seed tower must not be a pad along the quadrant edge. This requirement is approximately same as the one that the cluster centroid be at least $5^{\circ}$ away from the quadrant $\phi$ boundary.

\section{Track Associated to an EM cluster}

CTC and VTPC tracks reconstructed in 3 dimension are extrapolated to the depth $Z=190 \mathrm{~cm}$ of approximate shower maximum, and are examined if they match with the electron culster centroid in $\eta-\phi$ space. The best matched CTC and VTPC track are selected.

\section{Identification Variables}

The variables used for plug electron identification are listed below.

\section{(1) $H A D / E M$}

A ratio of the hadronic energy to the electromagnetic energy of the cluster. Figure 4.10 shows the $H A D / E M$ ratio for electrons from the $Z \rightarrow e e$ and for EM clusters in the jet sample.

\section{(2) Lateral shower shape: $\chi_{3 \times 3}^{2}$}

A $\chi^{2}$ obtained by comparing observed lateral shower shape with the predicted shape from test beam electrons. The shape comparison is performed in 2 dimension within total 9 pads $(\Delta \eta \times \Delta \phi=3 \times 3)$ in which most of the EM shower energy is contained. Figure 4.11 shows the distrubtions of $\chi_{3 \times 3}^{2}$ for electrons in the $Z \rightarrow$ ee events and EM clusters in the jet sample.

\section{(3) VTPC hit occupancy: $f_{V T P C}$}

The ratio of the number of hits recorded in the VTPC wires to the expected number of 
hits. This variable is already introduced for conversion removal in the central electron analysis. This quantity is used to make sure the existence of a VTPC track associated with a plug EM cluster. Requiring this value to be high, $\pi^{0}$ 's, $\gamma^{\text {'s }}$ and outer conversions are removed. Figure 4.12 shows distributions of the VTPC hit occupancy for electrons in the $Z \rightarrow e e$ events and EM clusters in the jet sample.

\section{(4) Track position matching}

The distance between an EM cluster centroid and a track position propagated to the approximate shower maximum $(Z=190 \mathrm{~cm})$. The difference in $\phi$ direction, $\Delta \phi$, and in the radial distance, $\Delta R$, are calculated. The $R$ is measured from the beam axis. Both CTC and VTPC tracks are considered in this analysis. Figure 4.13 shows the distributions of (a) the CTC $\phi$ matching, (b) the CTC radial distance matching, (c) the VTPC $\phi$ matching, and (d) the VTPC radial distance matching, for electrons in the $Z \rightarrow e e$ events and EM clusters in the jet sample.

\section{Isolation}

To get a further reduction of the ordinary QCD backgrounds, an isolation variable is defined:

$$
I_{\mathrm{S}}=\frac{E_{\mathrm{T}}(R=0.4)-E_{\mathrm{T}}^{\mathrm{EM}}(e)}{E_{\mathrm{T}}^{\mathrm{FN}}(e)} .
$$

In addition, we impose the track isolation that the number of CTC tracks with $P_{\mathrm{T}}>5$ $\mathrm{GeV} / c$ pointing to an $\mathrm{EM}$ cluster must be $\leq 1$. This is meant to remove conversion electrons. For historical reasons, the definition of $I_{\mathrm{S}}$ (Equation 4.8) is different from the one used in the central electron analysis. Since only the electron EM $E_{\mathrm{T}}$ is subtracted from the total $E_{\mathrm{T}}$ in a cone around the electron, a high $E_{\mathrm{T}}$ electron with some shower leakage into the hadronic part look less isolated than actual. This effect is taken into account by dividing by $E_{\mathrm{T}}^{\mathrm{CAI}}(e)$. One can subtract $E_{\Gamma}^{\mathrm{HAD}}(e)$ instead, as for the central electrons. However, a hadron shower is much larger than an EM shower, so a hadronic energy in the electron cluster may be overlapped from hadron showers from hadrons around 
the electron. Therefore subtracting $E_{\mathrm{T}}^{\mathrm{HAD}}(e)$ makes an electron look more isolated than actual. There is no decisive reason to choose either definition of the isolation variable. In this analysis, the definition of the isolation $I_{\mathrm{S}}$ is chosen merely for historical reasons. Figure 4.14 shows the isolation variable $I_{\mathrm{S}}$ for the electrons from ISAJET Monte Carlo $t \bar{t}$ events with the CDF detector simulation program, CDFSIM. The top quark mass is $90 \mathrm{GeV} / c^{2}$ for the plot.

\section{Definition of the Plug Electron Selection}

The requirements on the plug electrons in the top search are as follows:

1. Fiducial cuts

2. $E_{\mathrm{I}}^{\mathrm{EM}}>15 \mathrm{GeV}(>30 \mathrm{GeV}$ for the channel PEMI)

3. $I_{\mathrm{S}}<0.1$

4. Identification cuts

$$
\begin{aligned}
& -H A D / E M<0.1 \\
& -\chi_{3 \times 3}^{2}<10 \\
& -f_{V T P C}>0.7 \\
& - \text { CTC or VTPC track matching } \\
& \qquad \phi \phi<0.04 \text { radian and } \Delta R<6 \mathrm{~cm} \text { for the CTC track } \\
& \quad \Delta \phi<0.08 \text { radian and } \Delta R<8 \mathrm{~cm} \text { for the VTPC track. }
\end{aligned}
$$

The $E_{\mathrm{T}}$ threshold is raised to $30 \mathrm{GeV}$ for the PEMI channel because of trigger considerations. This type of events has to fire the plug electron trigger to be recorded in the data. The plug electron trigger with the $E_{\mathrm{T}}$ threshold set at $23 \mathrm{GeV}$ is found to be $50 \%$ efficient for the $30 \mathrm{GeV}$ offline $E_{\text {l }}$ threshold. 


\subsection{Central Muon Selection}

Basically, a muon can be identified by the presence of a CTC track with a consistent energy deposition in the calorimeter as a minimum ionizing particle. Therefore muon detection coverage is extended beyond the central muon chamber (CMU) coverage to $|\eta|<1.2$ which is covered by the CTC and the calorimeter. Muon momentum is measured from a CTC track. In the region of $|\eta|<0.63$, the CMU chambers are located outside of the calorimeter. In this region, the track segment in the chamber is also used to identify a muon by examining a match with the CTC track. Muon candidates with or without hits in the CMU are called CMUOs (Central MUon Objects) or CMIOs (Central Minimum Ionizing Objects), respectively. CMIOs are not necessarily restricted in the region of $0.63<|\eta|<1.2$, since there are $\phi$ cracks between the CMU chambers. The energy deposition by muons in the EM and HAD calorimeter is measured in a test beam [61] to be $E M_{\mu} \simeq 0.3 \mathrm{GeV}$ and $H A D_{\mu} \simeq 2 \mathrm{GeV}$, respectively (see Figure 4.15).

In the actual muon analysis codes at the reconstruction stage, all tracks with $P_{\mathrm{T}}>\mathbf{0 . 2}$ $\mathrm{GeV} / c$ are categorized as CMIOs regardless of the energy deposition in the calorimeters. Those in the CMIOs with the matched CMU segment are then called CMUOs.

\section{Track Quality Cuts}

In order to make sure that a track comes from a hard collision interaction and is not misreconstructed in the CTC, a set of track quality cuts are imposed on the track of a muon candidate. Two parameters are introduced, $D_{0}$ and $Z_{0}$. The $D_{0}$ is a radial distance of the track at the closest point to the beam axis, or the impact parameter. The $Z_{0}$ is the $z$ coordinate of the track at the closest point to the beam axis. We use the following track quality cuts:

- A track must be reconstructed in 3 dimension.

- $D_{0}<0.5 \mathrm{~cm}$

- $\left|Z_{\text {event }}-Z_{0}\right|<5.0 \mathrm{~cm}$. 
The efficiency of these requirements for real muons is known to be quite high ( $>99 \%)$ from a $W \rightarrow e \nu$ sample.

\section{Definition of the Fiducial Region}

For CMIOs which are identified using the calorimeter information, the fiducial cuts are defined to ensure that the measurement with the calorimeter is correct.

- $|\eta|<1.2$

- The CTC track of a CMIO is propagated to the CES, $R=184 \mathrm{~cm}$. The track must be at least $2.5 \mathrm{~cm}$ away from a $\phi$ boundary of the wedge.

- An absolute value of $z$ coordinate of the track at the CES radius must be $>9 \mathrm{~cm}$. This avoids tracks passing through the $90^{\circ}$ crack.

- A CMIO whose track passes through the wedge of number 5 and has a $z$ coordinate of the track at the CES radius larger than $210 \mathrm{~cm}$ is rejected. There is a pipe for liquid helium which cools the CDF solenoid.

No explicit fiducial cut is applied to CMUOs because the CMU chamber geometry naturally avoids a muon passing through the cracks.

\section{Identification Variables}

The identification variables for central muons are listed below.

(1) Energy of the tower through which a muon traverses: $E M_{\mu}$ and $H A D_{\mu}$

The $E M_{\mu}$ and $H a d_{\mu}$ are the electromagnetic and hadronic energy of the tower through which a muon traverses. Figures 4.16 and 4.17 show the $E M_{\mu}$ and the $H A D_{\mu}$ distributions for muons in $Z \rightarrow \mu \mu$ events and muon background in a jet sample.

(2) Position matching : $D_{x}^{\mu}$

This variable quantifies how good the matching between the CTC track and the track 
segment in the muon chamber is. This is relevant only for CMUOs. The CTC track is first propagated to the CMU radius, $R=348.4 \mathrm{~cm}$. The CTC track and the CMU track segment are separately fit to straight lines in the transverse plane ( $x-y$ plane). The $D_{X}^{\mu}$ is a difference in fitted intercept of the lines. Figure 4.18 shows the $D_{X}^{\mu}$ distributions for muons in the $Z \rightarrow \mu \mu$ and muon background in the jet sample. As mentioned in $\$ 3.1 .4$, this matching gets worse due to multiple scattering processes. The uncertainty in $D_{X}^{\mu}$ coming from this effect is given as

$$
\Delta D_{X}^{\prime \prime}=\frac{15(\mathrm{GeV} / c \cdot \mathrm{cm})}{P_{\jmath}} .
$$

This is obtained by multiplying the effective length of the calorimeter of $181 \mathrm{~cm}$ to Equation (3.10).

\section{Isolation}

We use a calorimetric isolation and a track isolation:

$$
\begin{aligned}
& E_{\mathrm{T}}^{\text {cone }}(\mathrm{GeV})=E_{\mathrm{T}}(R=0.4)-E_{\mathrm{T}}^{\mu}, \\
& P_{\mathrm{T}}^{\text {cone }}(\mathrm{GeV} / c)=P_{\mathrm{T}}(R=0.4)-P_{\mathrm{T}}^{\mu},
\end{aligned}
$$

where $E_{\mathrm{T}}(R=0.4)$ is the total transverse energy within a cone radius of 0.4 in $\eta$ - $\phi$ space, $E_{\mathrm{T}}^{\mu}$ is the transverse energy of the tower through which a muon traverses, $P_{\mathrm{T}}(R=0.4)$ is the total transverse momentum within a cone radius of 0.4 in $\eta-\phi$ space, and $P_{\mathrm{T}}^{\mu}$ is the muon $P_{\top}$. Figure 4.19 shows the isolation distribution for central muons from ISAJET+CDFSIM $t \bar{t}$ Monte Carlo with $M_{\text {top }}=90 \mathrm{GeV} / c^{2}$.

\section{Definition of the Central Muon Selection}

The central muon selection is summarized as follows:

1. Track quality cuts

2. Fiducial cuts for CMIOs 
3. $P_{\mathrm{T}}>15 \mathrm{GeV} / c$

4. $E_{\mathrm{T}}^{\text {cone }}<5 \mathrm{GeV}$ and $P_{\mathrm{T}}^{\text {cone }}<5 \mathrm{GeV} / c$

5. Identification cuts

- $E M_{\mu}<2 \mathrm{GeV}, \operatorname{Had}_{\mu}<6 \mathrm{GeV}$ and $E M_{\mu}+\operatorname{Had}_{\mu}>0.1 \mathrm{GeV}$

$-D_{X}^{\mu}<10 \mathrm{~cm}$ for CMUOs.

The cut $E M_{\mu}+\operatorname{Had}_{\mu}>0.1$ is required because there was a bug in an offline muon reconstruction code which picked up a wrong tower where no track actually passed through.

\subsection{Event Topology Cuts}

As backgournds for the $t \bar{t}$ dilepton events, we can consider $c \bar{c}$, $b \bar{b}$ events, $Z \rightarrow \tau \tau$ events followed by the decays of both $\tau$ 's into $e \nu \nu$ or $\mu \nu \nu$, and $Z \rightarrow e e, \mu \mu$ especially for the dielectron and dimuon events. Ordinary QCD jet events or $W+$ jets events with one or more misidentified leptons can also leave the dilepton signature. These backgrounds are called "fake dilepton" events.

The lepton identification cut as described in the previous section is effective to remove fake dilepton events. The requirement of the high $P_{\Gamma}$ and the isolation greatly reduce the $c \bar{c}, b \bar{b}$ and fake dilepton events because leptons from these process have moderate $P_{\mathrm{T}}$ and usually less isolated due to particle activities from jets near the leptons.

On the other hand, the backgrounds such as the $Z \rightarrow \tau \tau$ events and the $Z \rightarrow e e$ or $\mu \mu$ events contain high $P_{T}$ isolated leptons. Since it is not possible to discriminate such backgrounds from the $t \bar{t}$ events based on only the lepton selection, one has to use some information which characterize the $t \bar{t}$ events.

Based on Monte Carlo study, a back-to-back cut requiring $\Delta \phi_{c: \mu}<160^{\circ}$ is imposed for $e \mu$ events in order to suppress the $b \bar{b}$ and $Z \rightarrow \tau \tau$ backgrounds, where $\Delta \phi_{e \mu}$ is the $e \mu$ azimuthal opening angle. In the background processes, $Z \rightarrow \tau \tau$ for instance, $\tau$ 's are 
emitted back-to-back because the intermediate $Z$ is produced mainly at low transverse momentum and therefore gives rise to $\tau$ 's with nearly balancing transverse momenta. Then the mass of $\tau$ is too light to make the correlation between the direction of $\tau$ 's flight and that of the daughter lepton small enough to survive the back-to-back cut. On the other hand, leptons from the top quarks are not strongly correlated in directions due to the heavy mass of the top quark, which allows a large portion of the $t \bar{t}$ events pass the back-to-back cut. This back-to-back cut is also effective to reject fake lepton backgrounds. Figure 4.20 shows a $\Delta \phi$ distribution between a muon and a jet in an inclusive muon sample. We see a peak around $180^{\circ}$. Considering that fake leptons which are mainly hadrons tend to come from jets, a lepton plus one fake lepton will have a back-to-back configuration.

This back-to-back cut is effective to reject dielectron and dimuon events from DrellYan process by the same reason for the $Z \rightarrow \tau \tau$ process. However this background is so large that the back-to-back cut is not sufficient. The Drell-Yan process includes both $Z$ and $\gamma$ as the intermideate bosons. The high $P_{\Gamma}$ cut on leptons, however, reduces the continume process via the $\gamma$. The resulting dielectron and dimuon events mostly consist of the events from $Z$ decays. Further reduction of this background is obtained by applying a dilepton invariant mass window cut around the $Z$ mass and a missing transverse energy cut. The $Z \rightarrow e e / \mu \mu$ events have small $\mathbb{W}_{\mathrm{T}}$ because they don not' produce $\nu$ in the reaction while the $t \bar{t}$ dilepton events have larger $\psi_{\mathrm{T}}$ due to the existence of $\nu$ 's produced in the weak decays of the top quarks.

The following event kinematical and topological cuts (we simply call them topology cut) are used:

- $e \mu$ events

$$
-\Delta \phi_{c \mu}<160^{\circ}
$$

- $e e / \mu \mu$ events

$$
-\Delta \phi_{\ell \ell}<160^{\circ}
$$


- reject events with $75<M_{\ell}<105 \mathrm{GeV} / c^{2}$

$-\mathbb{E}_{\mathrm{T}}>20 \mathrm{GeV}$.

A scatter plot Figure 4.21 shows $\Delta \phi_{e \mu}$ versus the least energetic lepton $P_{\mathrm{T}}$ for $e \mu$ events from the $Z \rightarrow \tau \tau, b \bar{b}$ and $t \bar{t}$ processes. Figure 4.22 shows $M_{\ell \ell}$ distributions for $e e / \mu \mu$ events from the $Z, \gamma \rightarrow e e, \mu \mu$ and $t \bar{t}$ processes. Figure 4.23 is a scatter plot of $\mathscr{E}_{\mathrm{T}}$ versus $\Delta \phi_{\ell \ell}$ for dilepton events from the $Z, \gamma \rightarrow e e, \mu \mu, b \bar{b}$ and $t \bar{t}$ processes. The $t \bar{t}$ events in these plots are Monte Carlo data with $M_{\text {tup }}=90 \mathrm{GeV} / c^{2}$.

For dimuon events, cosmic ray events are also to be removed. Cosmic ray events with a high $P_{\mathrm{T}}$ muon are characterized by the two tracks with a back-to-back configuration in 3 dimensional space. We require

$$
\begin{aligned}
& \text { - }\left|\eta_{\mu 1}+\eta_{\mu 2}\right|>0.1 \\
& \text { - } \Delta \phi_{\mu \mu}<178.5^{\circ} .
\end{aligned}
$$

The second requirement on the azimuthal opening angle is always satisfied because of the back-to-back cut in this analysis. More sophisticated algorithm to identify cosmic ray events is developed [62]. It rejects muons (1) not attached to the event vertex, (2) back-to-back of the poorly reconstructed track (a track traversing from outer to inner in the CTC has a bad reconstruction quality resulting in a 2 dimensional track or having few hits in the CTC), (3) back-to-back of the good track but with the velocity $v / c$, obtained by fitting the two back-to-back track as a single track, greater than 0.5 (For cosmic muons, the back-to-back two tracks are created by a fast single muon resulting in $v / c \simeq 1$ ), and (4) without coinsidence of the timing of $p \bar{p}$ beam crossing. Identified cosmic ray events with this algorithm are removed according to their run number and event number from the dimuon sample. 


\subsection{Result of the Selection}

After applying the $E_{\mathrm{T}}$ or $P_{\mathrm{T}}$ cut, isolation cut and identification cut on leptons in the fiducial region, $4 e \mu$ ( $1 \mathrm{CEMI}$ and $3 \mathrm{PEMU}$ ), $271 e e$ and $112 \mu \mu$ events are found in the data. After applying the invariant mass window cut for the dielectron and dimuon events, they are reduced to $55 e e$ and $15 \mu \mu$ events. A scatter plot of the electron $E_{\mathrm{T}}$ versus the muon $P_{\mathrm{T}}$ is shown in Figure 4.24 (a) for the $4 e \mu$ events. Figure 4.24 shows a scatter plot of $E_{\mathrm{T}}$ or $P_{\mathrm{T}}$ of the least energetic lepton versus $\Delta \phi_{e \mu}$ for the 4 $e \mu$ events. Figure 4.25 shows invariant mass distributions for the $272 e e$ and $112 \mu \mu$ events in comparison with $Z, \gamma \rightarrow e e, \mu \mu$ Monte Carlo distributions. It indicates the dielectron and dimuon data are consistent with the $Z, \gamma \rightarrow e e, \mu \mu$ process. Figure 4.26 shows scatter plots of $\Delta \phi_{\ell \ell}$ versus $\not_{\mathrm{T}}$ for the $55 e e$ and the $15 \mu \mu$ events after the mass cut.

There is only one $e \mu$ event, CEMI, passing all the selection cuts. This event is the same one as reported in the previous publication on the top quark search using central $e \mu$ events [19]. This event has an isolated electron with $E_{\mathrm{T}}$ of $31.7 \mathrm{GeV}$ and an isolated opposite sign muon with $P_{\mathrm{T}}$ of $42.5 \mathrm{GeV} / c$ with a dilepton azimuthal opening angle of $137^{\circ}$. Other characteristics of the event include the presence of a second muon candidate with $P_{\mathrm{T}}$ of $9.9 \mathrm{GeV} / c$ in the forward muon detector, and two small jets with observed $E_{\mathrm{T}}$ of 14 and $5 \mathrm{GeV}$. The event characteristics are summarized in Table 4.2. 
Run 19250, Event 20435.

\begin{tabular}{|c|c|c|c|c|}
\cline { 2 - 5 } \multicolumn{1}{c|}{} & Charge & $\begin{array}{c}P_{\cdot} \\
{[\mathrm{GeV} / c]}\end{array}$ & $\eta$ & $\begin{array}{c}\phi \\
{[\text { degrees }]}\end{array}$ \\
\hline Central Electron & + & 31.7 & -0.81 & 132 \\
Central Muon & - & 42.5 & -0.80 & 269 \\
Forward Muon & + & 9.9 & -2.0 & 98 \\
Jet 1 & & 14 & 1.1 & 341 \\
Jet 2 & & 5 & -2.8 & 88 \\
\hline
\end{tabular}

Table 4.2: Characteristics of the top candidate event. The observed calorimeter $E_{\mathrm{T}}$ is used in the $P_{\text {. }}$ column for the electron and jet clusters. 


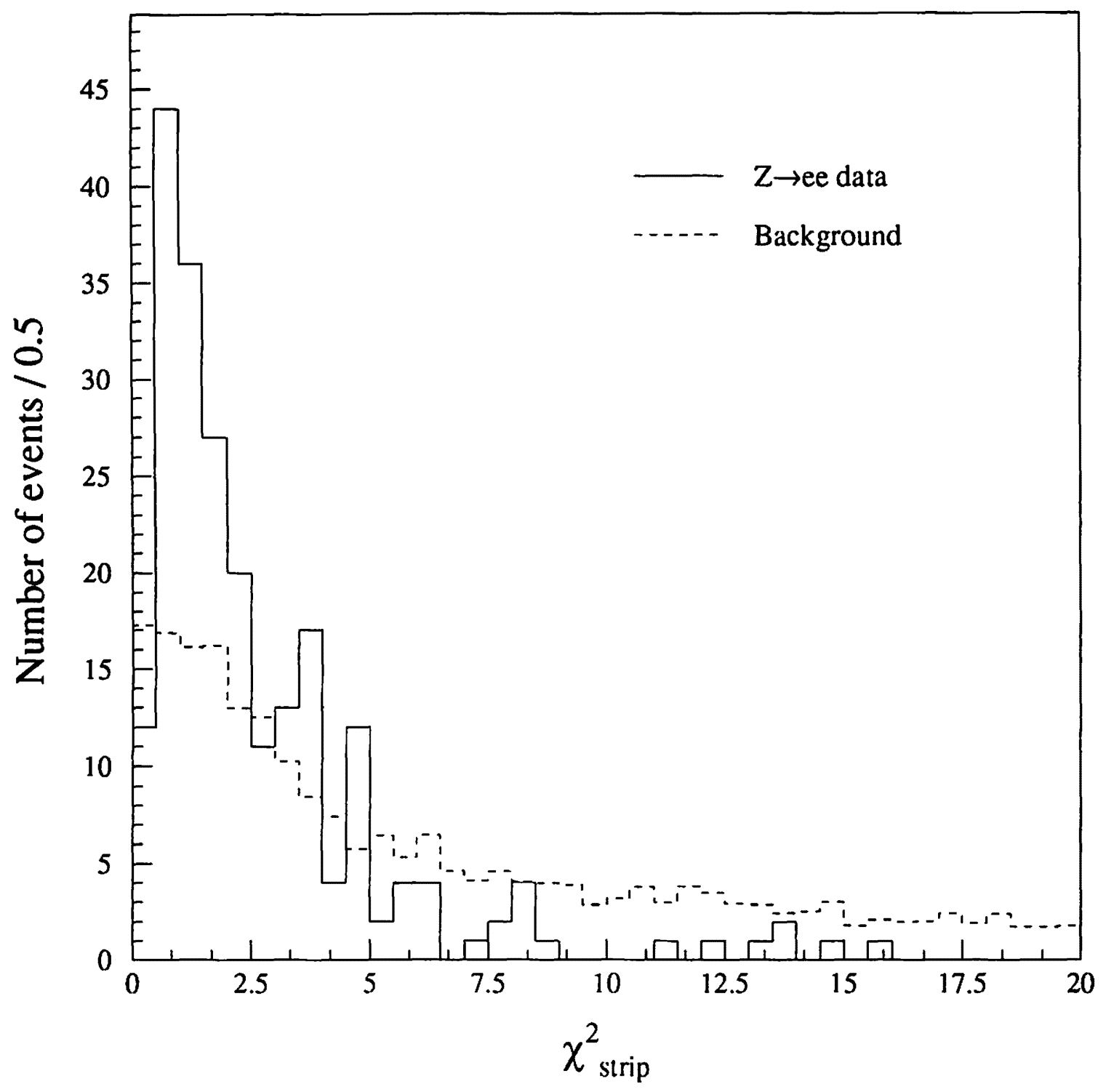

Figure 4.1: The $\chi_{\text {strip }}^{2}$ for central electrons from $Z \rightarrow e e$ and jet samples. 


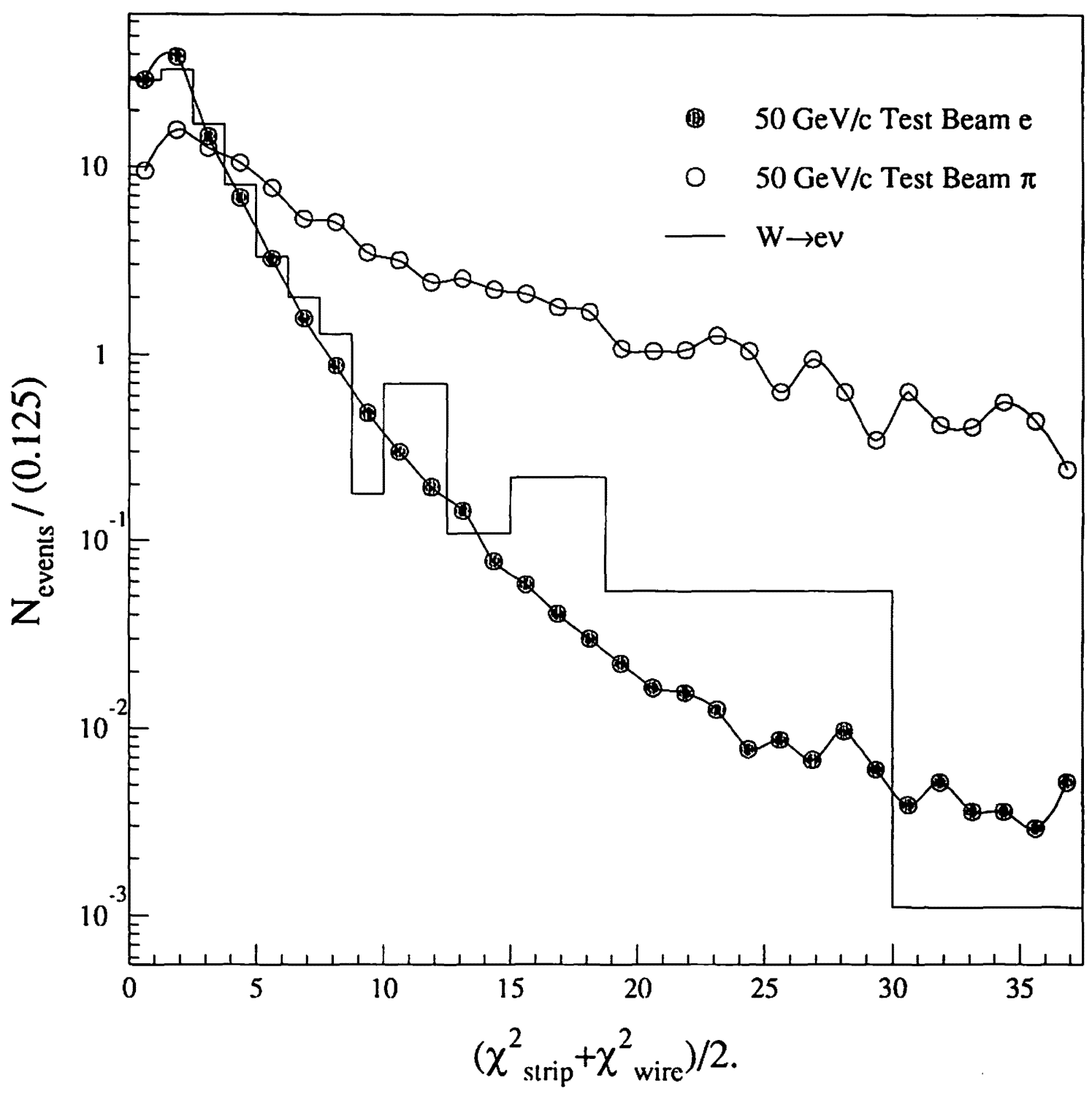

Figure 4.2: The average strip chamber $\chi^{2}=\left(\chi_{\text {strip }}^{2}+\chi_{\text {wire }}^{2}\right) / 2$ distributions for test beam electrons, test beam charged pions and $W$ electrons. 


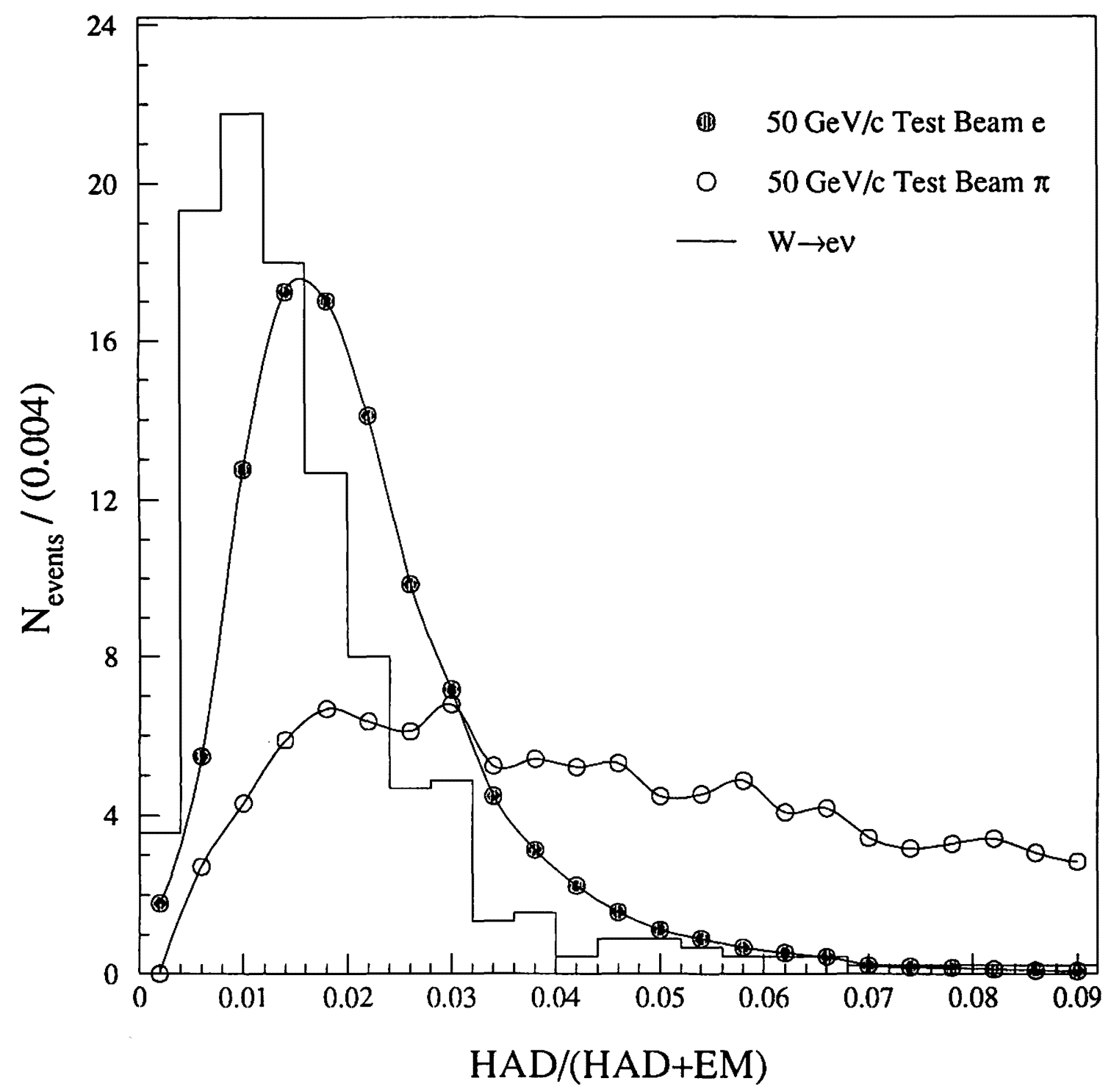

Figure 4.3: The $H A D /(E M+H A D)$ ratio for test beam electrons, test beam $\pi$ 's and electrons from $W \rightarrow e \nu$ events. 


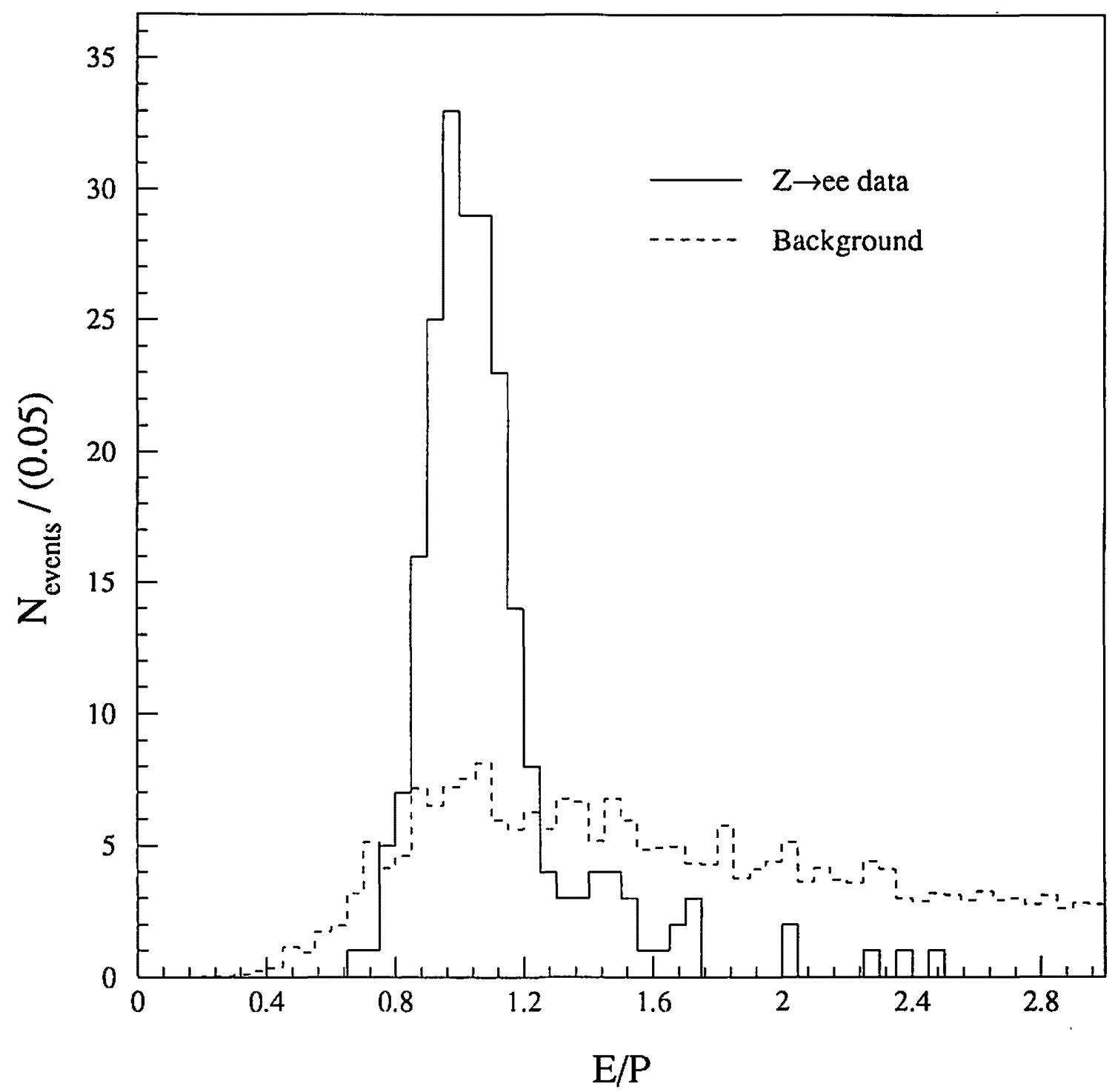

Figure 4.4: The ratio of the calorimeter energy and the momentum, $E / P$, for central electrons from $Z \rightarrow e e$ and jet samples. 

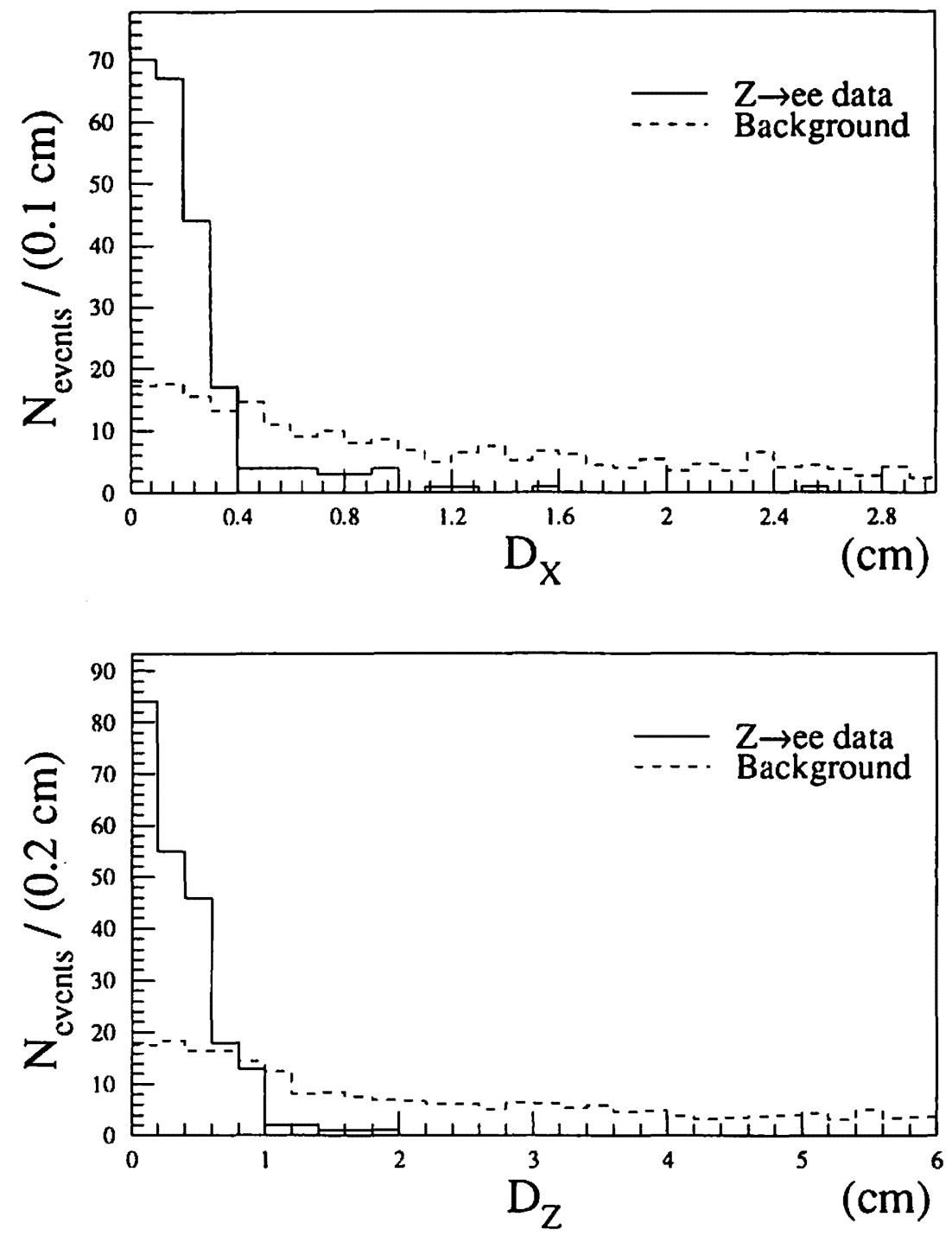

Figure 4.5: The position match in $R-\phi$ view $\left(D_{X}\right)$ and in $z$ direction $\left(D_{Z}\right)$ for central electrons from $Z \rightarrow e e$ and jet samples. 


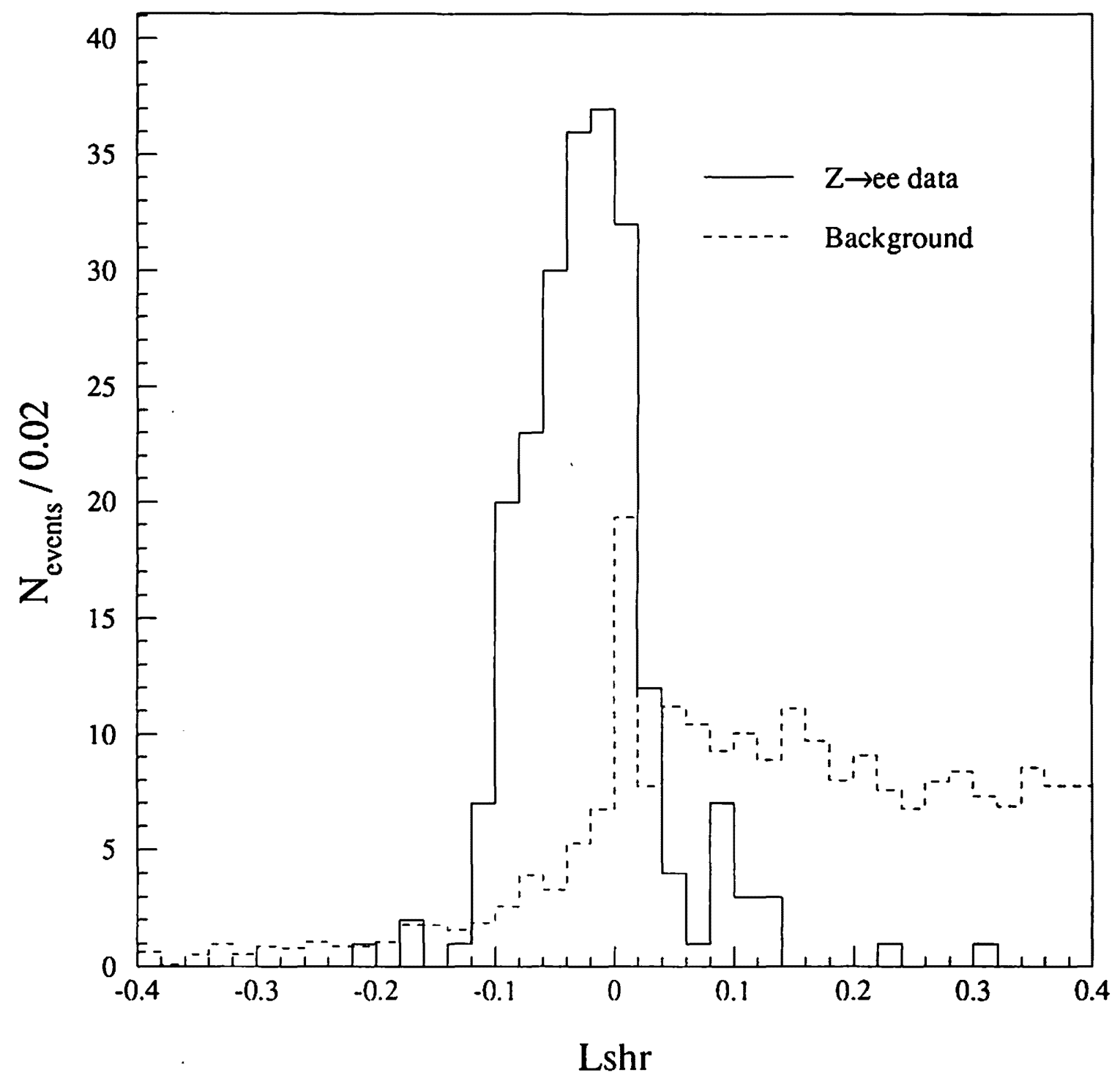

Figure 4.6: The lateral shower profile variable $\left(L_{\text {str }}\right)$ for central electrons from $Z \rightarrow e e$ and jet samples. 


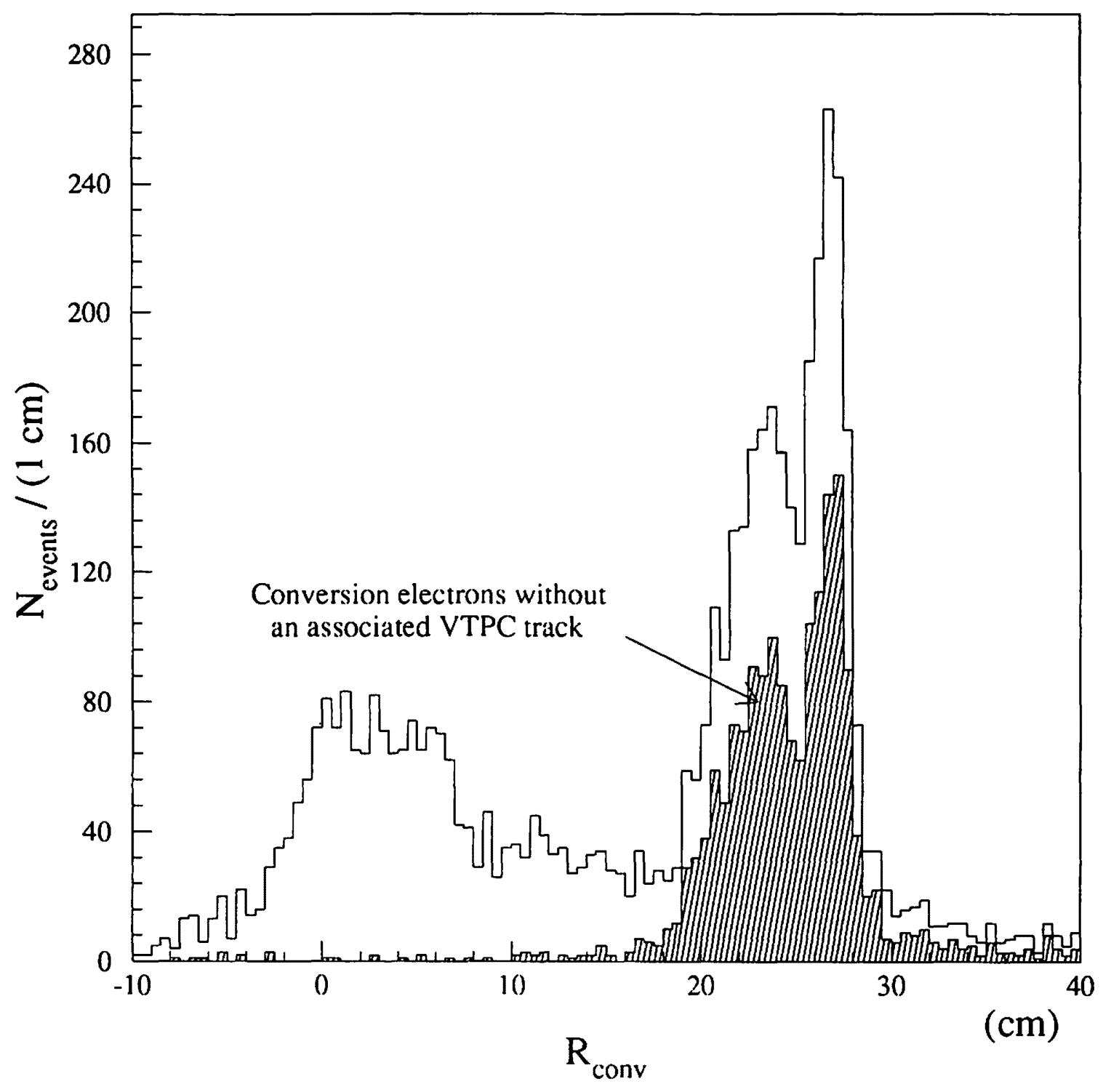

Figure 4.7: The radial distance of the conversion points from central conversion electron sample. 


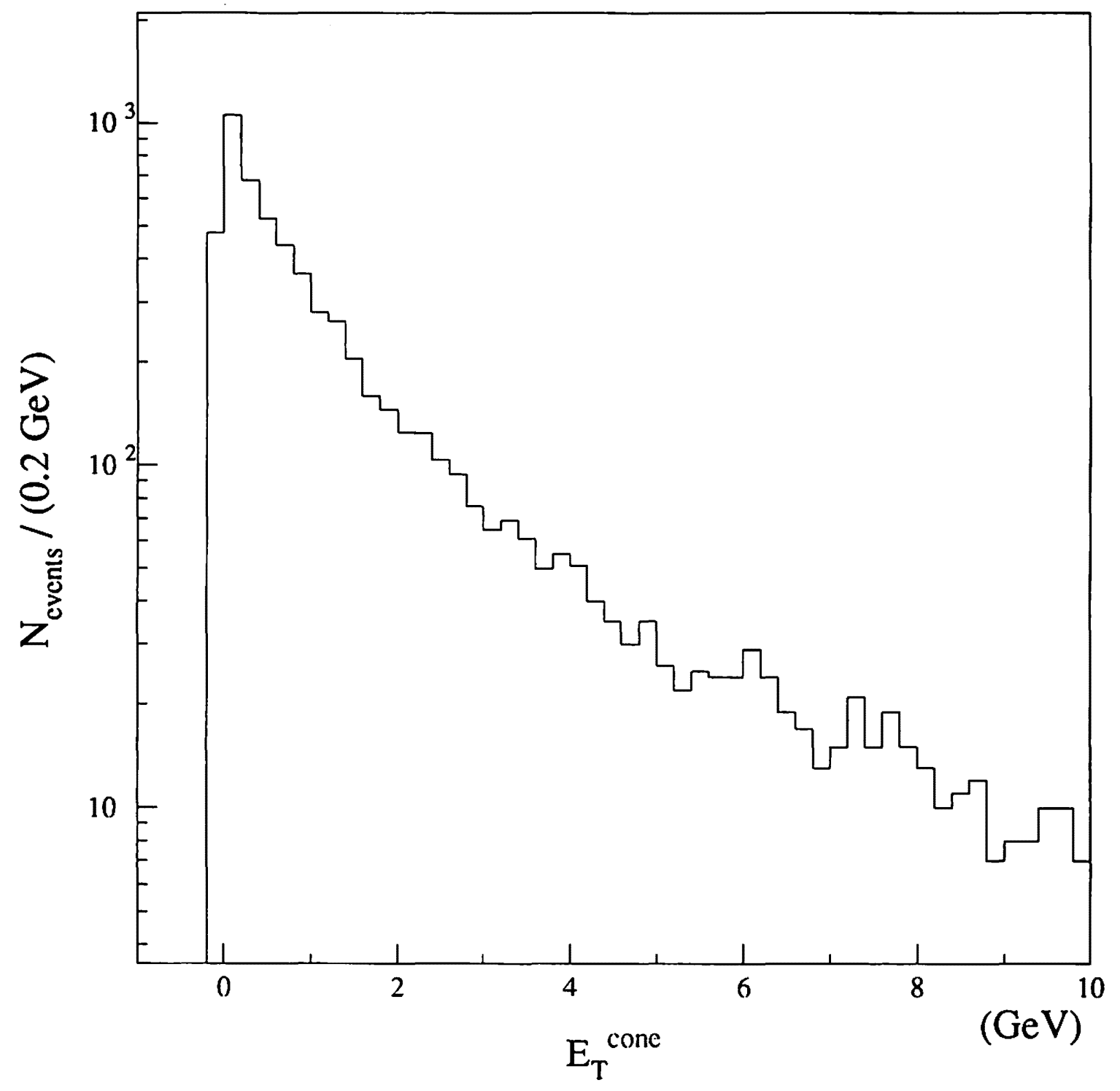

Figure 4.8: The isolation distribution for central electrons from the ISAJET+CDFSIM $t \bar{t}$ Monte Carlo with $M_{\mathrm{Lop}}=90 \mathrm{GeV} / c^{2}$. 


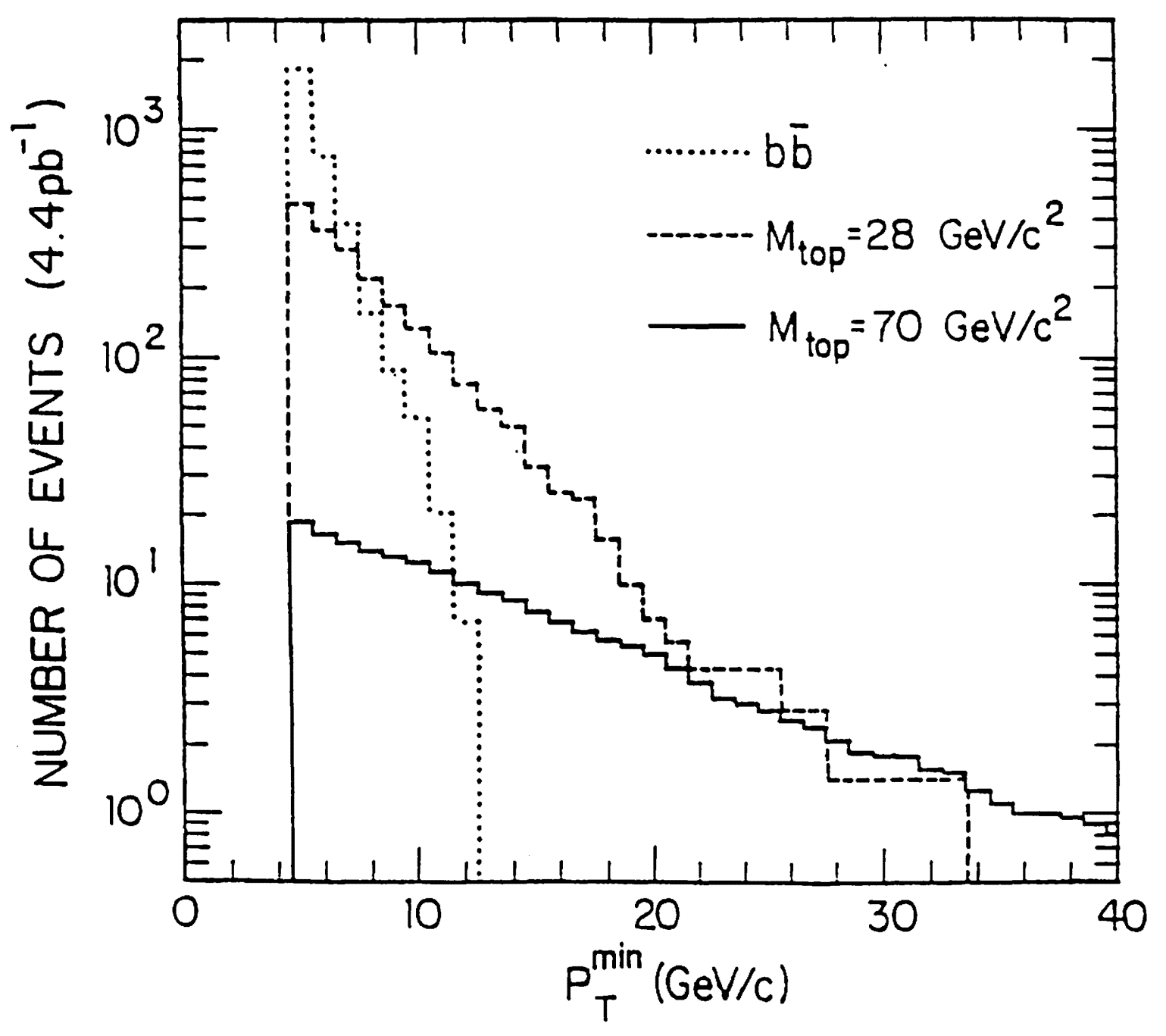

Figure 4.9: The predicted number of dilepton events as a function of common $E_{\mathrm{T}}\left(P_{\mathrm{T}}\right)$ threshold on both leptons from Reference [19]. 


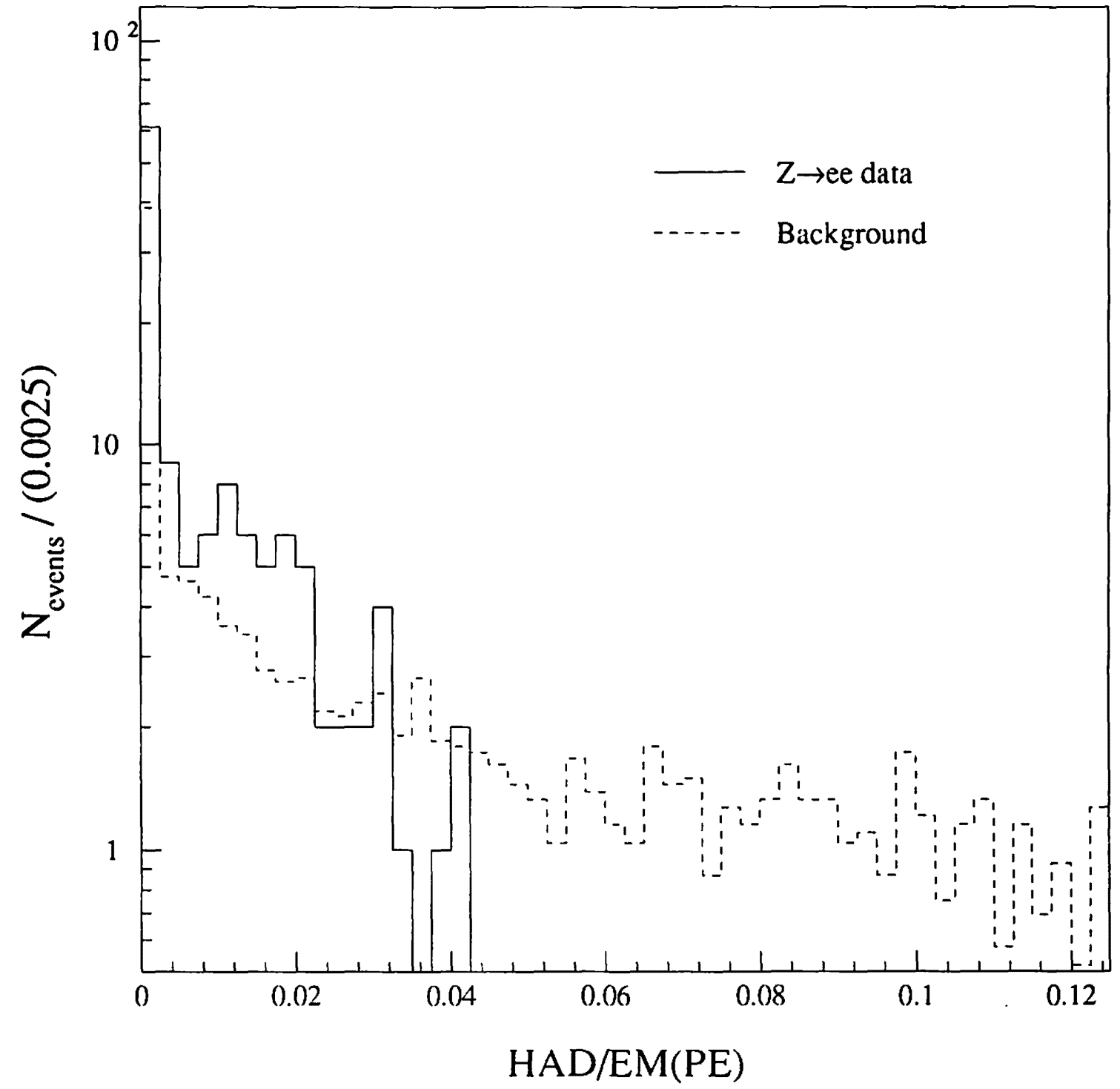

Figure 4.10: The ratio of the hadronic energy and the electromagnetic energy for plug electrons from $Z \rightarrow e e$ and jet samples. 


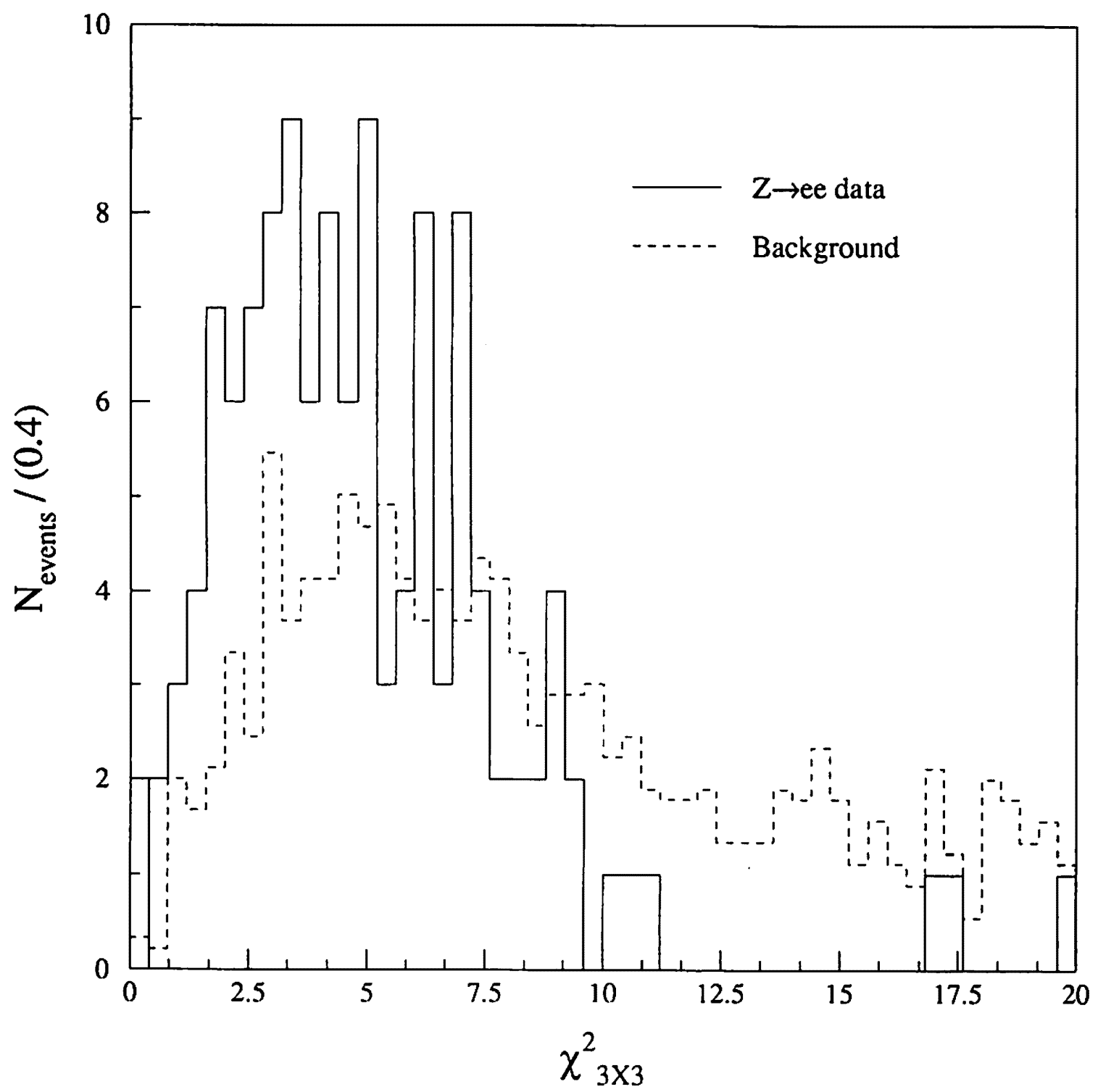

Figure 4.11: The lateral shape chi-squared of the EM shower, $\chi_{3 \times 3}^{2}$, for plug electrons from $Z \rightarrow e e$ and jet samples. 


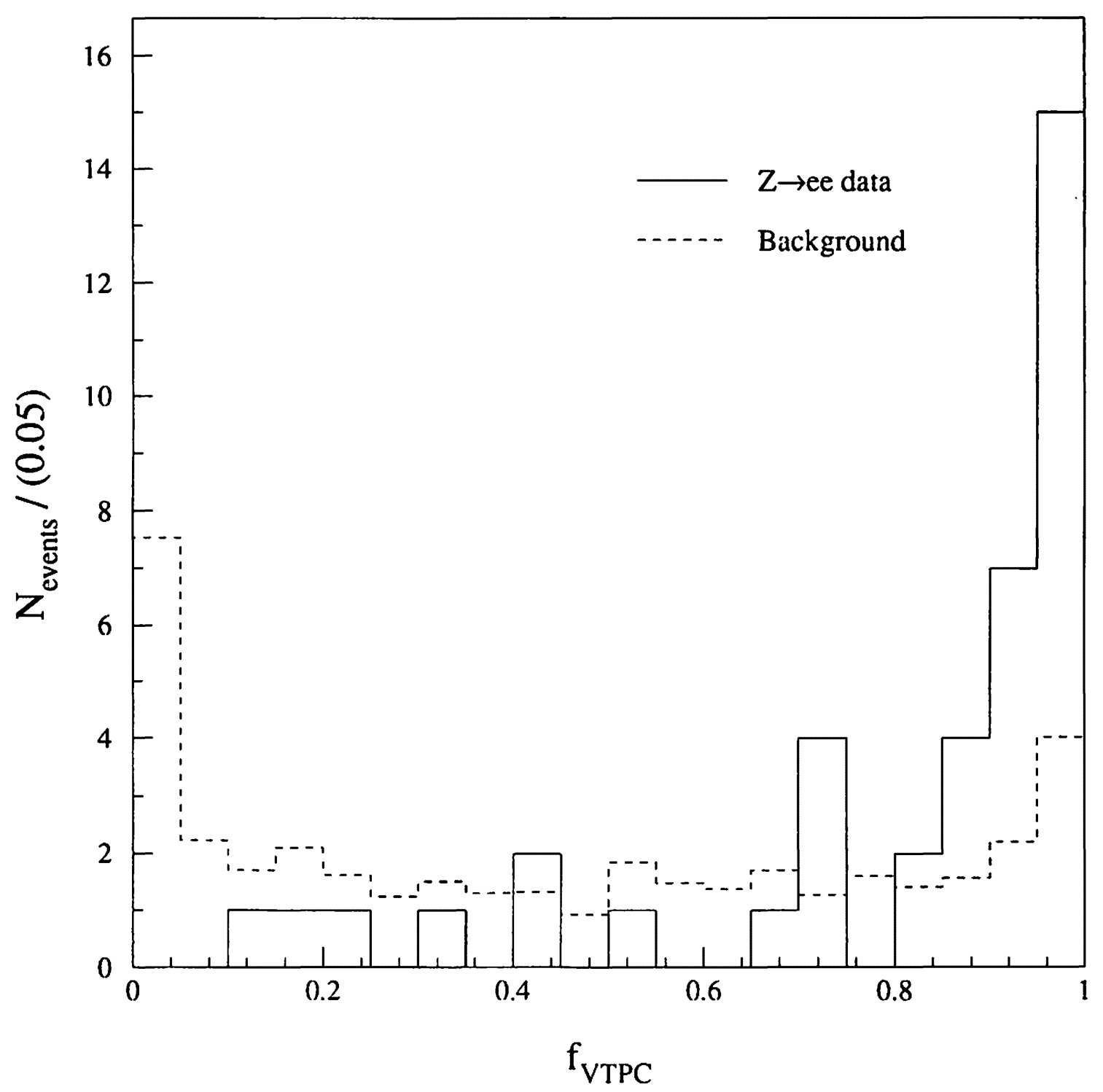

Figure 4.12: The VTPC hit occupancy, $f_{\vee T P C}$, for plug electrons from $Z \rightarrow e e$ and jet samples. 

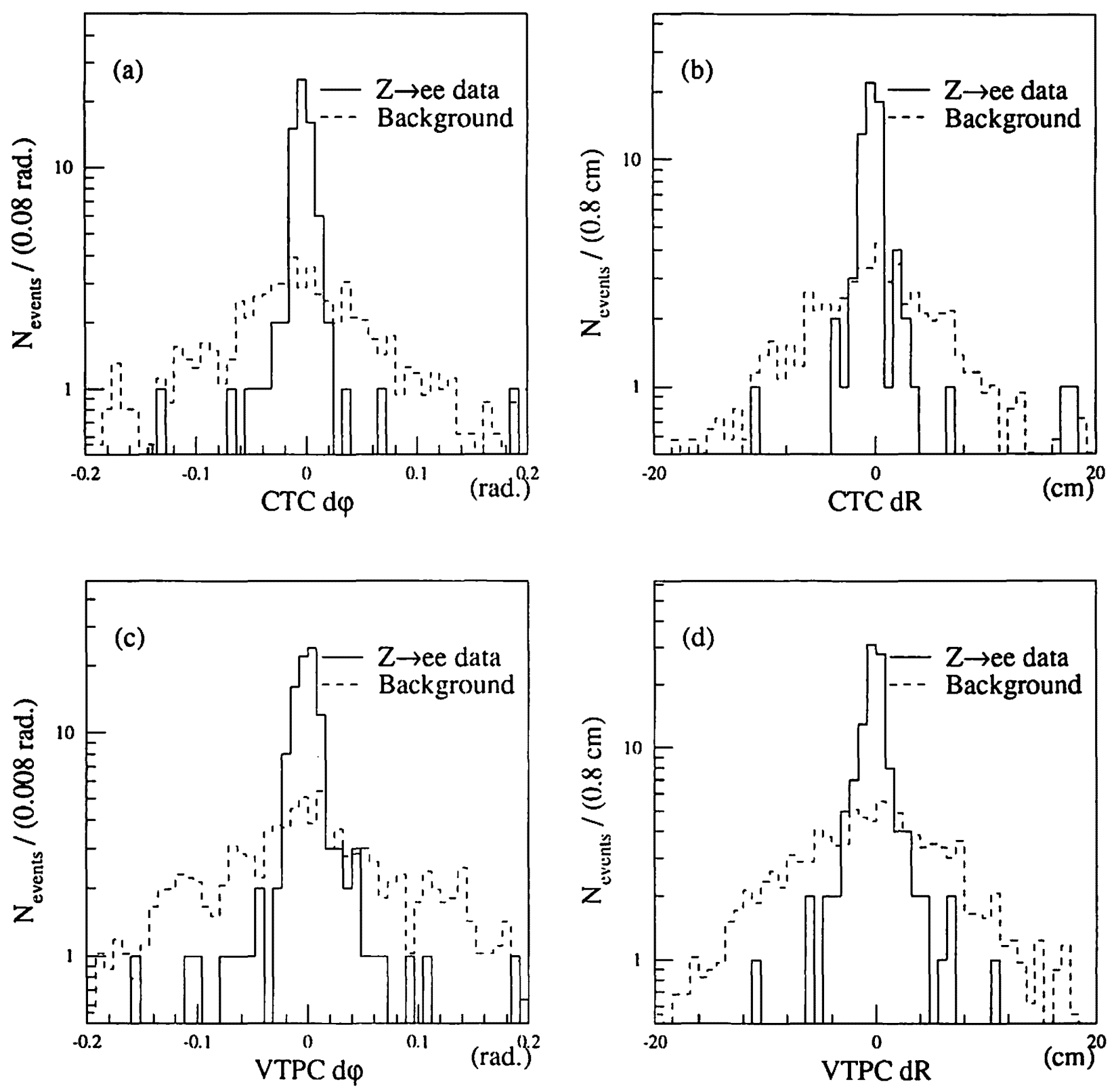

Figure 4.13: Track matching for plug electrons from $Z \rightarrow e e$ and jet samples. (a) The CTC $\phi$ matching, (b) the CTC radial distance matching, (c) the VTPC $\phi$ matching, and (d) the VTPC radial distance matching. 


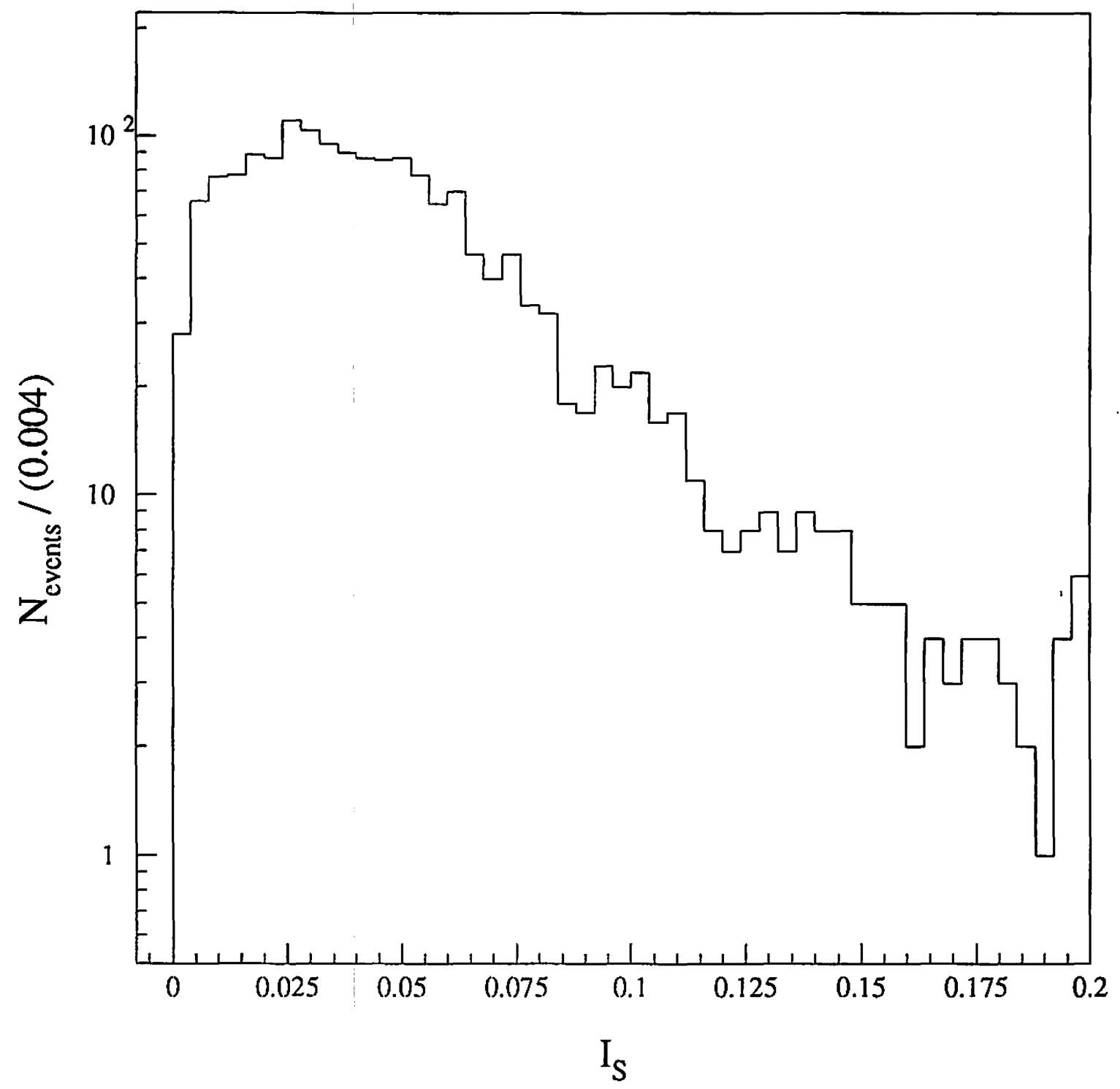

Figure 4.14: The isolation distribution for plug electrons from the ISAJET+CDFSIM $t \bar{t}$ Monte Carlo with $M_{\text {Lop }}=90 \mathrm{GeV} / c^{2}$. 

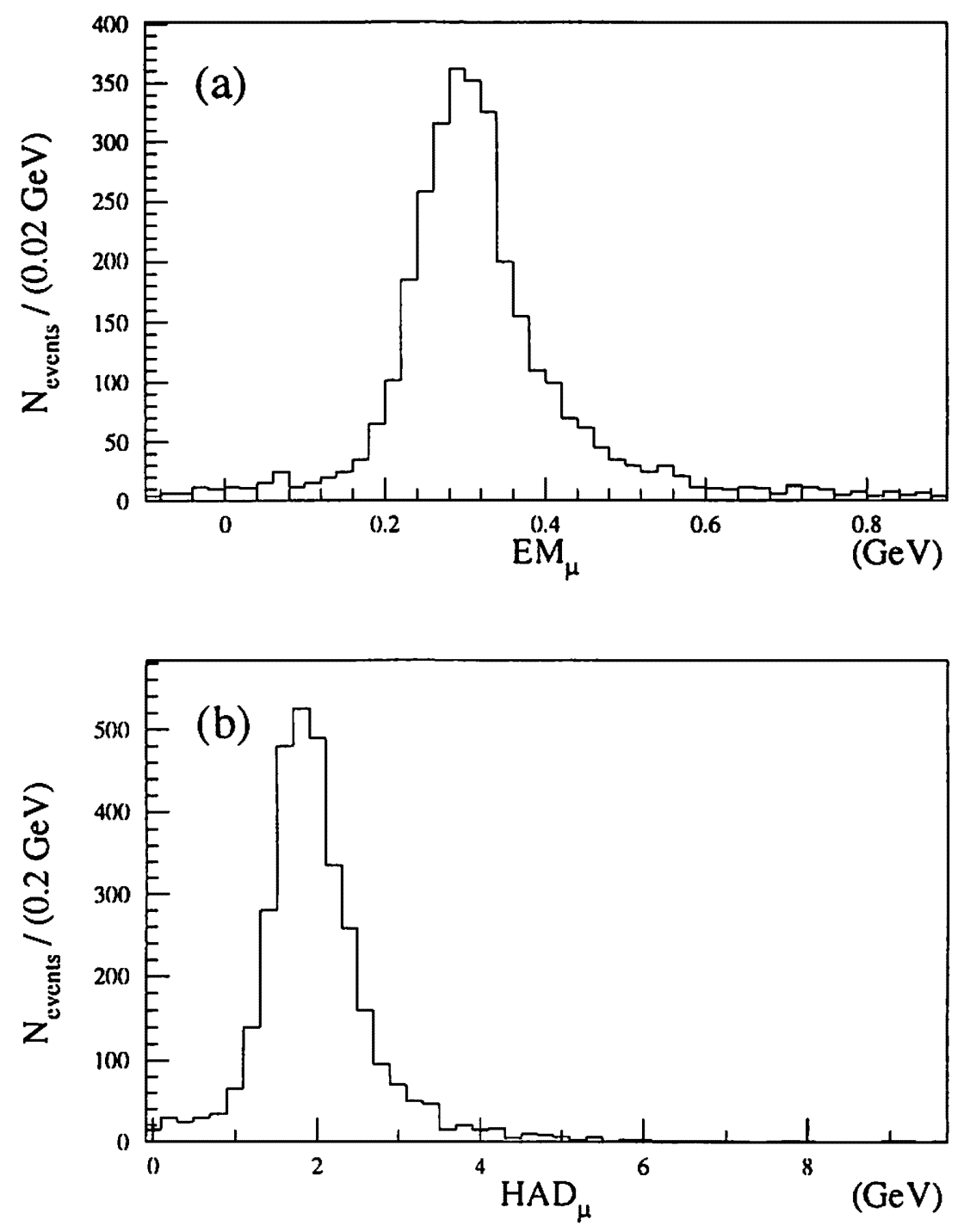

Figure 4.15: The energy distribution of the tower through which a muon traverses from test beam data. (a) The electromagnetic energy $E M_{\mu}$ and (b) the hadronic energy $H A D_{\mu}$. 


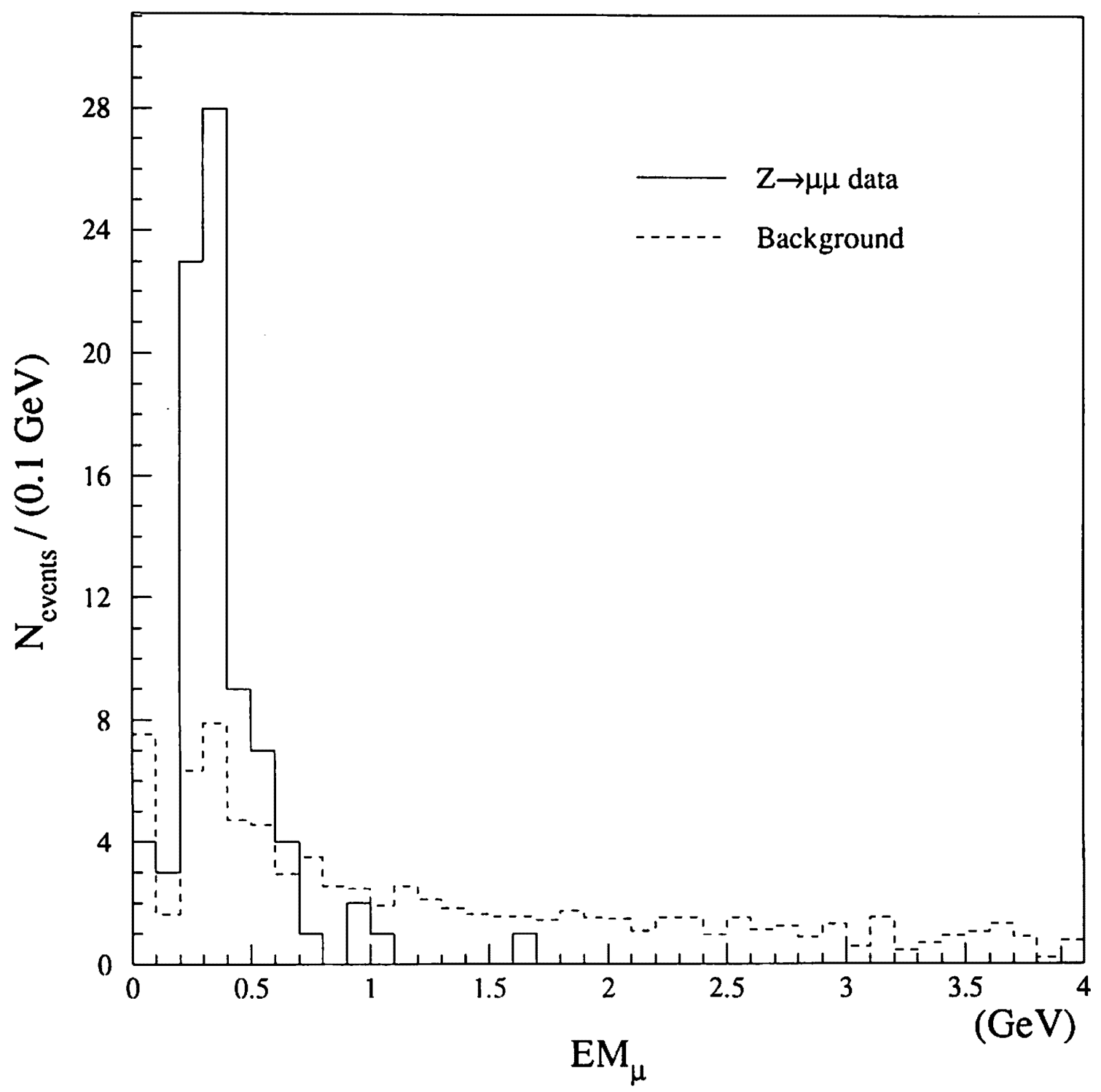

Figure 4.16: The electromagnetic tower energy $E M_{\mu}$ through which a muon traverses. The distributions from $Z \rightarrow \mu \mu$ and jet samples are shown. 


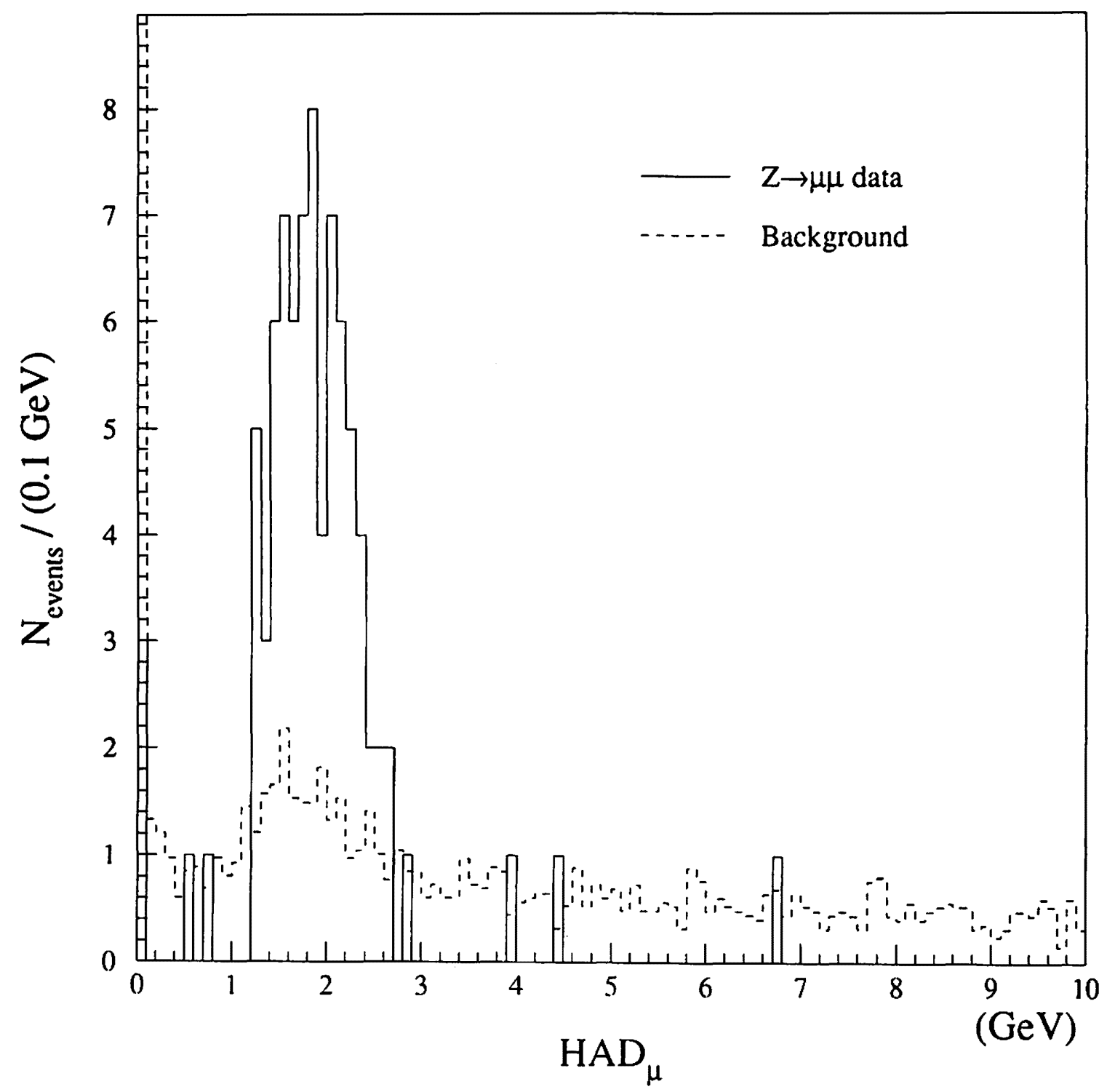

Figure 4.17: The hadronic tower energy $H A D_{\mu}$ through which a muon traverses. The distributions from $Z \rightarrow \mu \mu$ and jet samples are shown. 


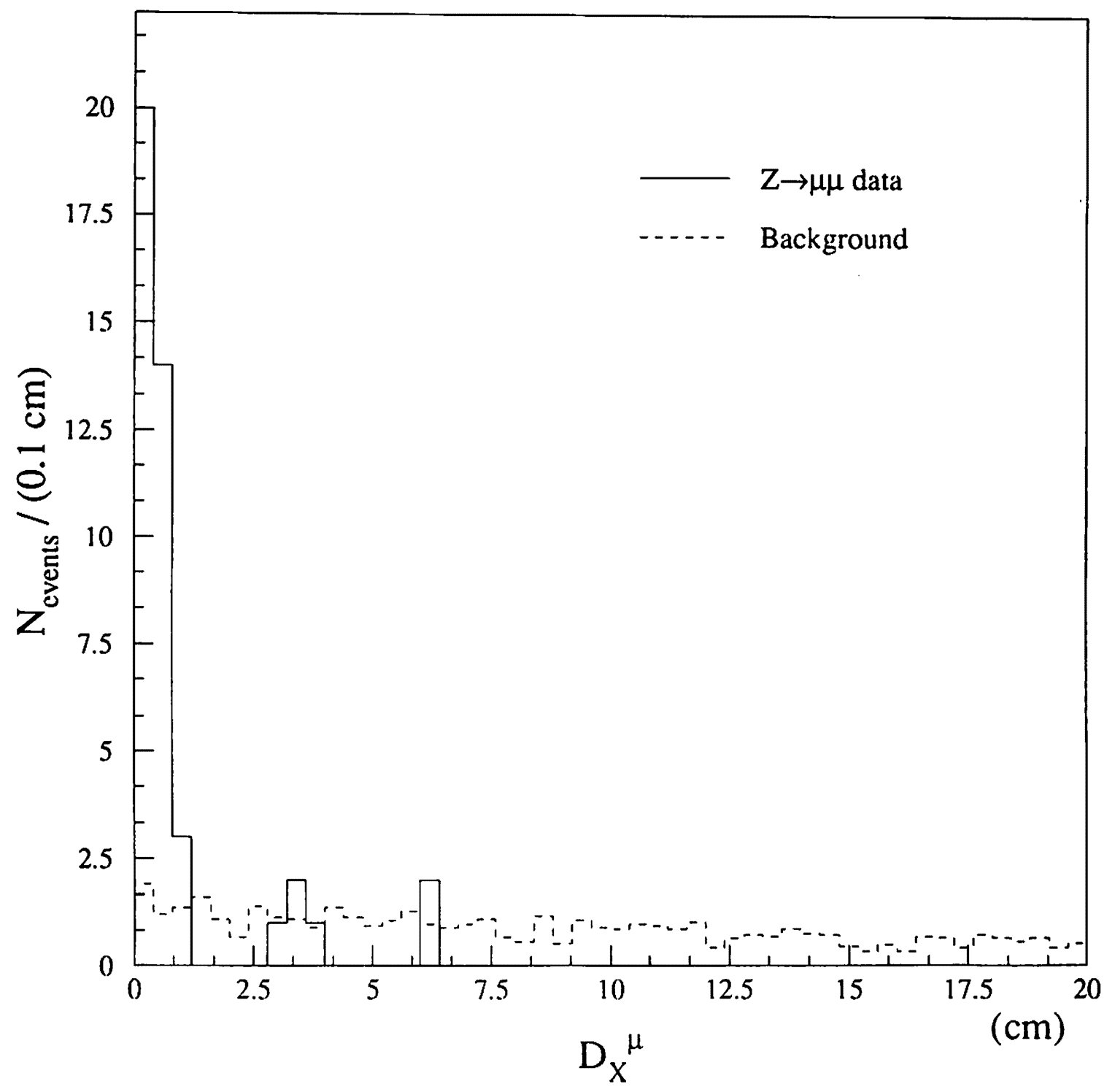

Figure 4.18: The position matching in $R-\phi$ view, $D_{X}^{\mu}$, between the CTC track and the CMU chamber hits for central muons from $Z \rightarrow \mu \mu$ and jet samples. 

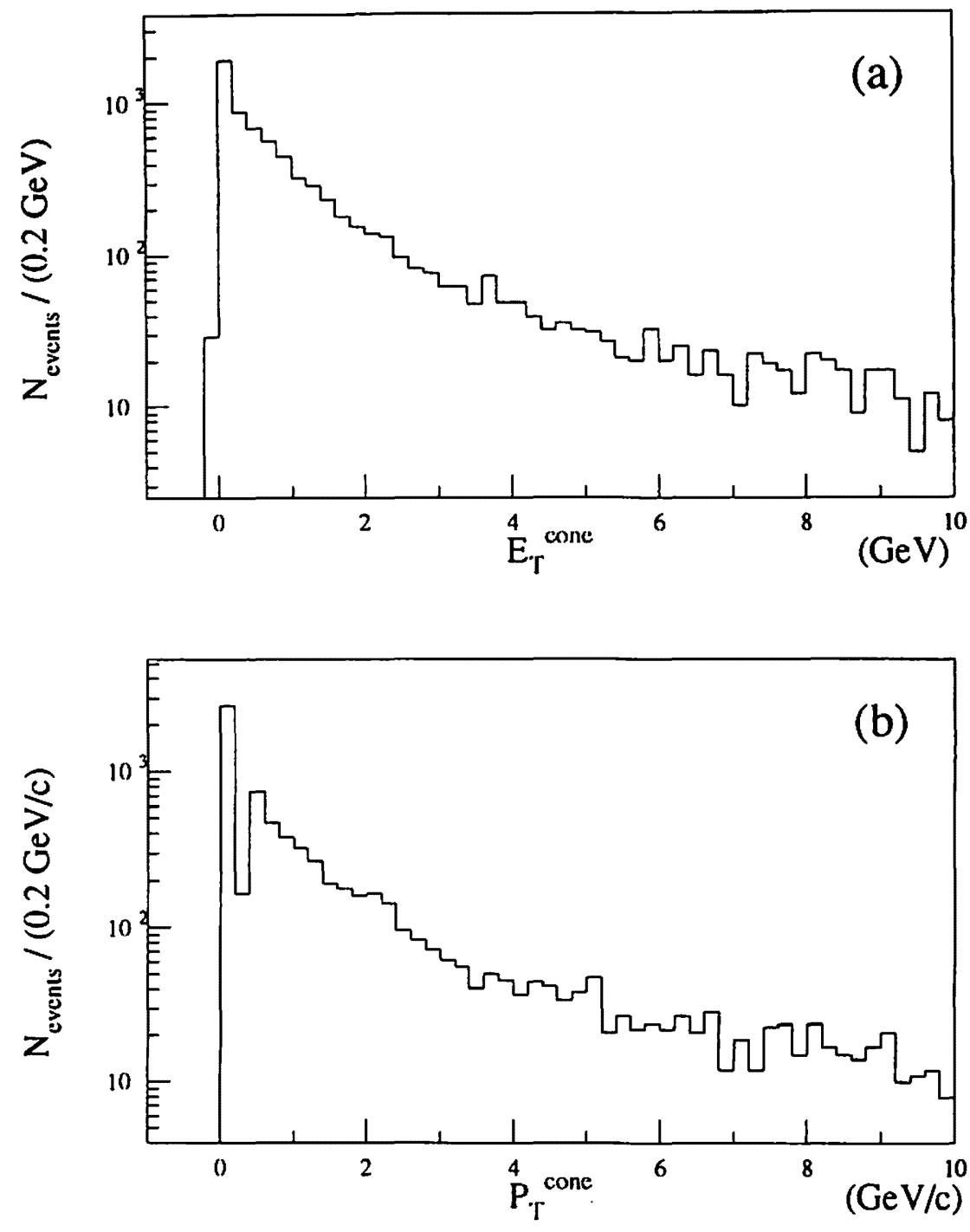

Figure 4.19: The distributions of (a) calorimeter isolation and (b) track isolation, for central muons from the ISAJET+CDFSIM Monte Carlo with $M_{\text {Lup }}=90 \mathrm{GeV} / c^{2}$. 


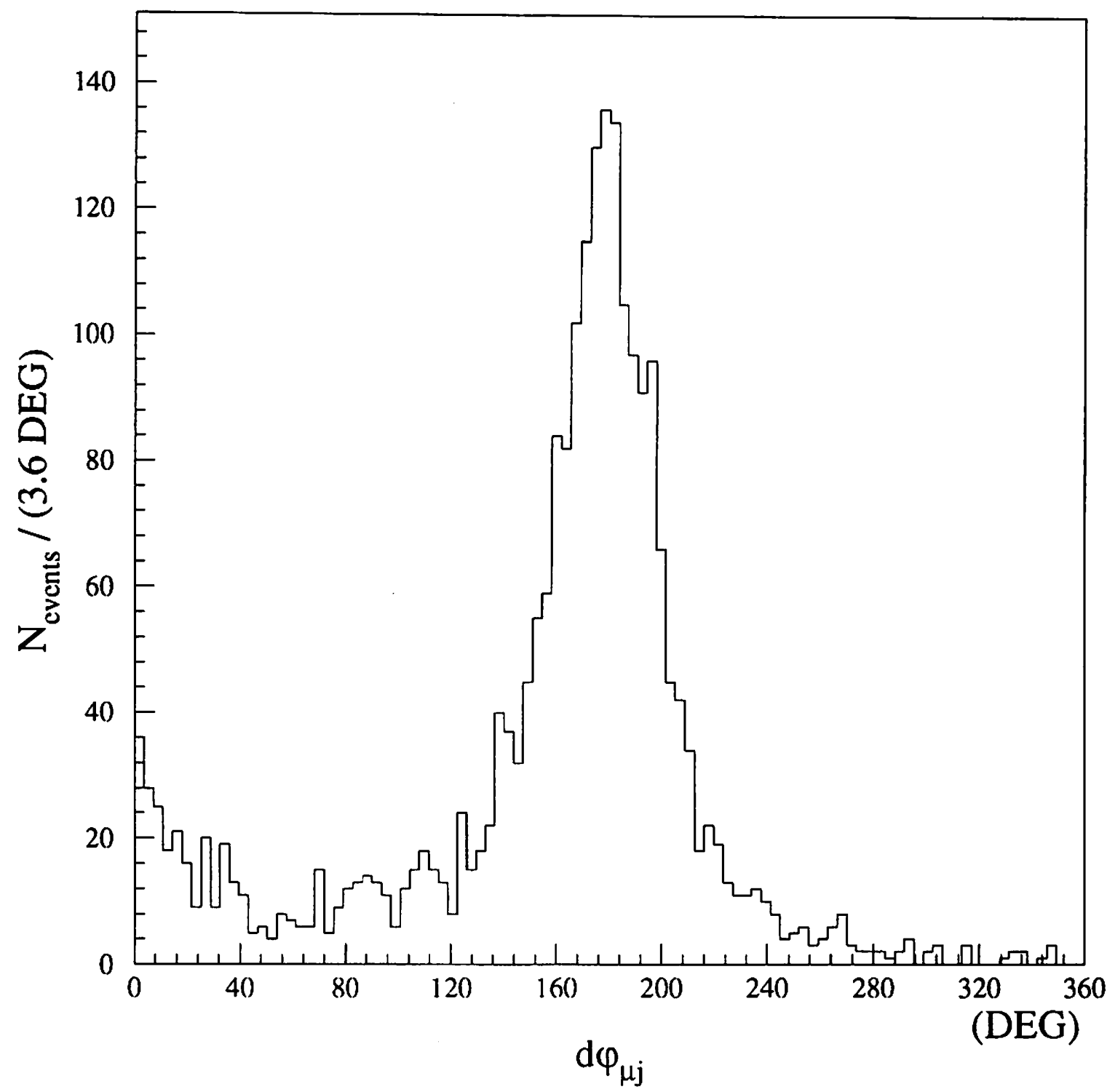

Figure 4.20: The opening angle $\Delta \phi$ between a muon and a jet in an inclusive muon sample. 

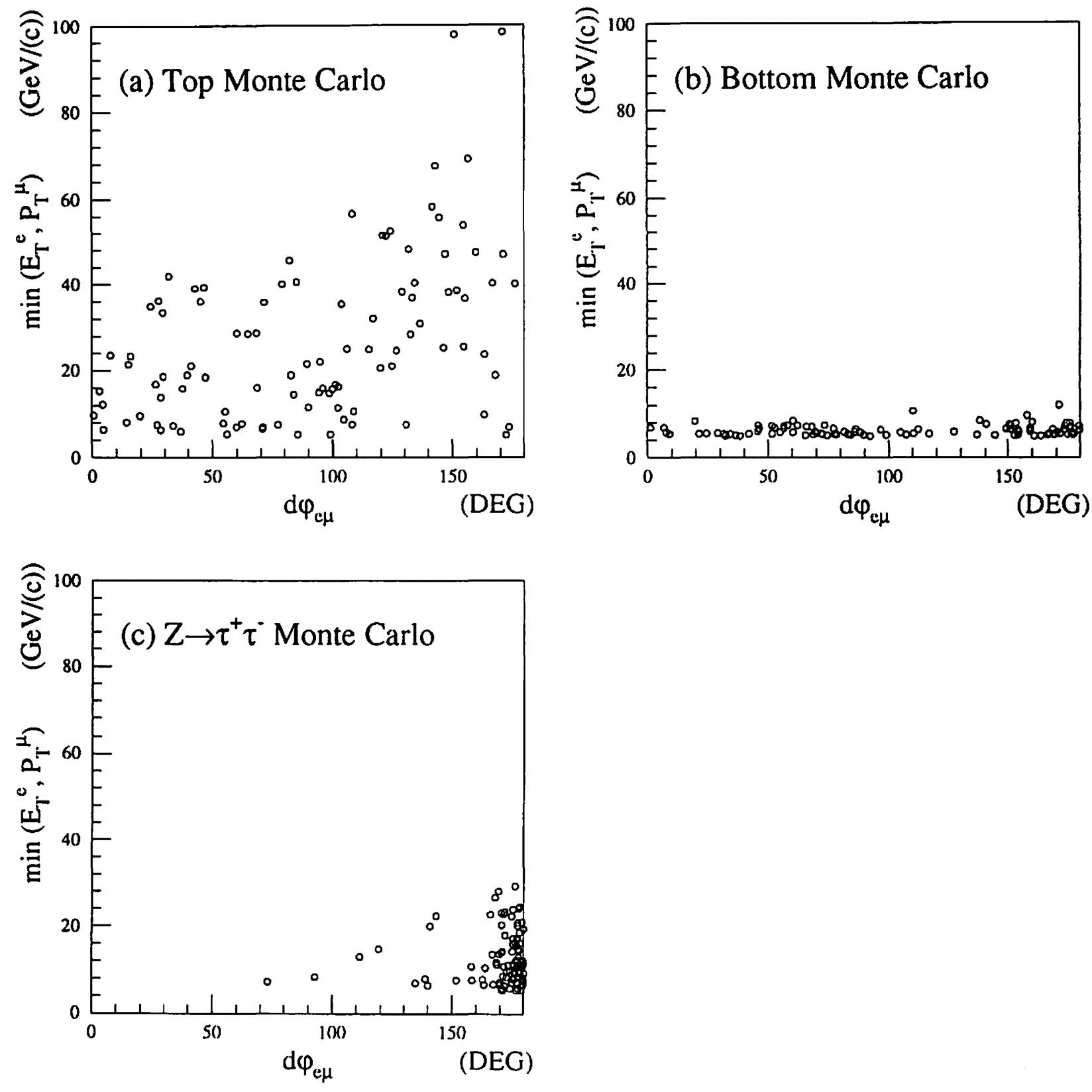

Figure 4.21: The azimuthal opening angle $\Delta \phi_{c ; \mu}$ between an electron and a muon for $e \mu$ events from (a) the $t \bar{t}$ Monte Carlo with $M_{\mathrm{Lop}}=90 \mathrm{GeV} / c^{2}$, (b) $b \vec{b}$ Monte Carlo, and (c) $Z \rightarrow \tau \tau$ Monte Carlo. 

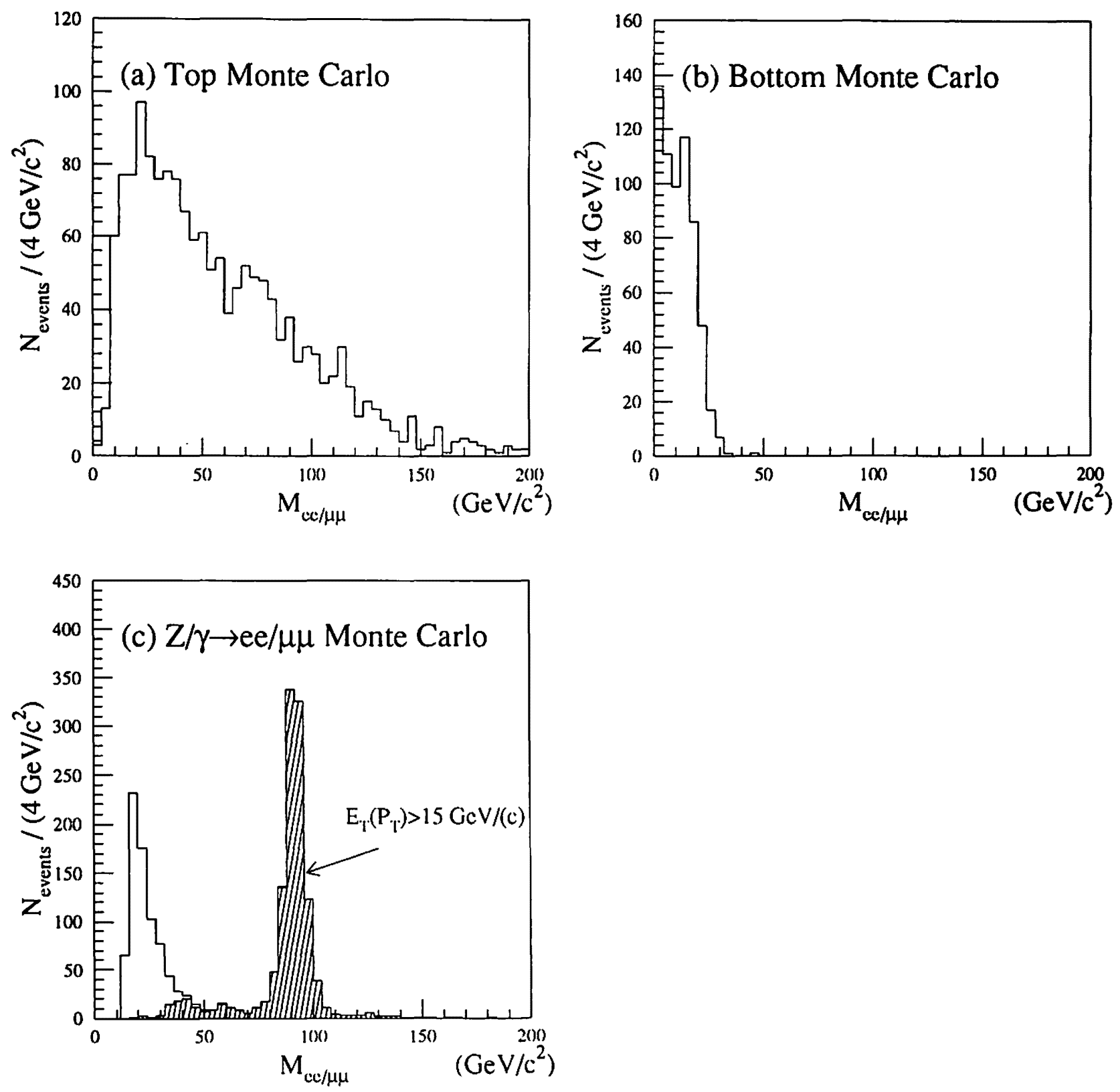

Figure 4.22: The invariant mass $M_{t i t}$ of dielectron or dimuon pairs from (a) the $t \bar{t}$ Monte Carlo with $M_{\text {top }}=90 \mathrm{GeV} / c^{2}$, (b) $b \bar{b}$ Monte Carlo, and (c) $Z, \gamma \rightarrow e e, \mu \mu$ Monte Carlo. 

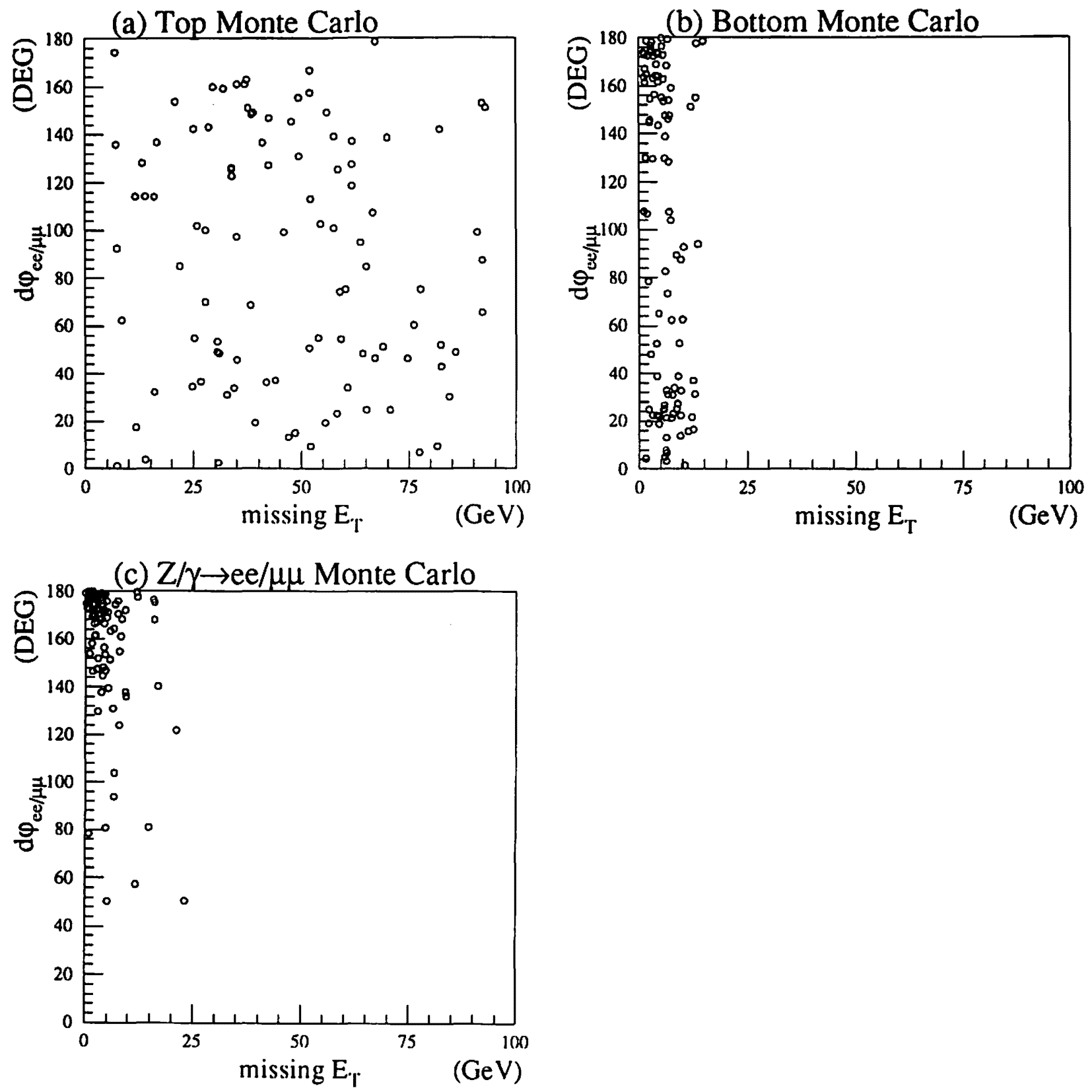

Figure 4.23: Scatter plots of $\Delta \phi_{\ell \ell}$ versus $E_{T}$ for $e e, \mu \mu$ events from (a) the $t \bar{t}$ Monte Carlo with $M_{\text {lop }}=90 \mathrm{GeV} / c^{2}$, (b) $b \bar{b}$ Monte Carlo, and (c) $Z, \gamma \rightarrow e e, \mu \mu$ Monte Carlo. 

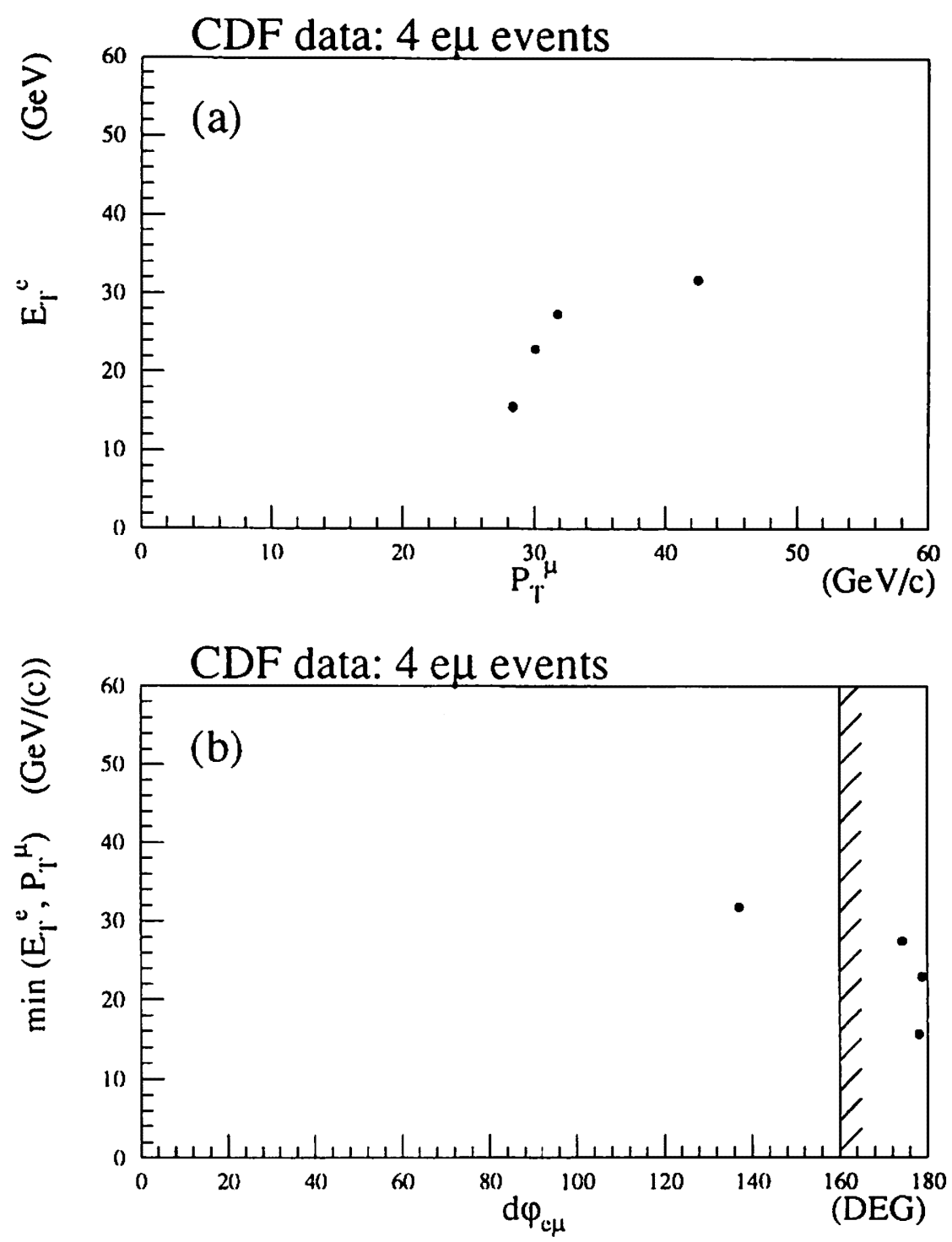

Figure 4.24: Scatter plots for the $4 e \mu$ events. (a) The electron $E_{\mathrm{T}}$ versus the muon $P_{\mathrm{T}}$. (b) The transverse energy or the tranverse momentum of the least energetic lepton versus the opening angle between $e$ and $\mu$. 

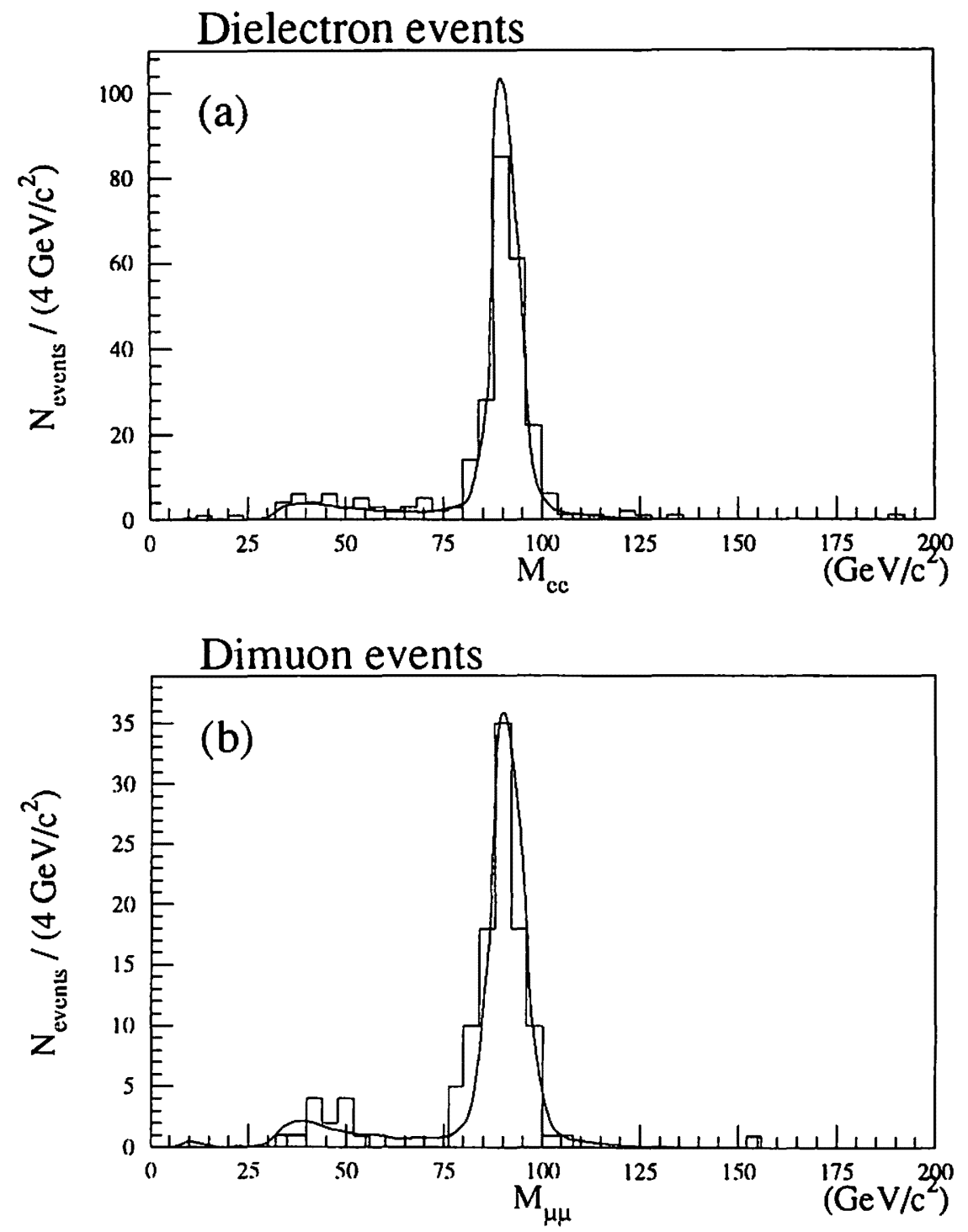

Figure 4.25: The invariant mass distributions for the dielectron and dimuon CDF data (histograms) together with the $Z, \gamma \rightarrow e e, \mu \mu$ Monte Carlo (curves). 

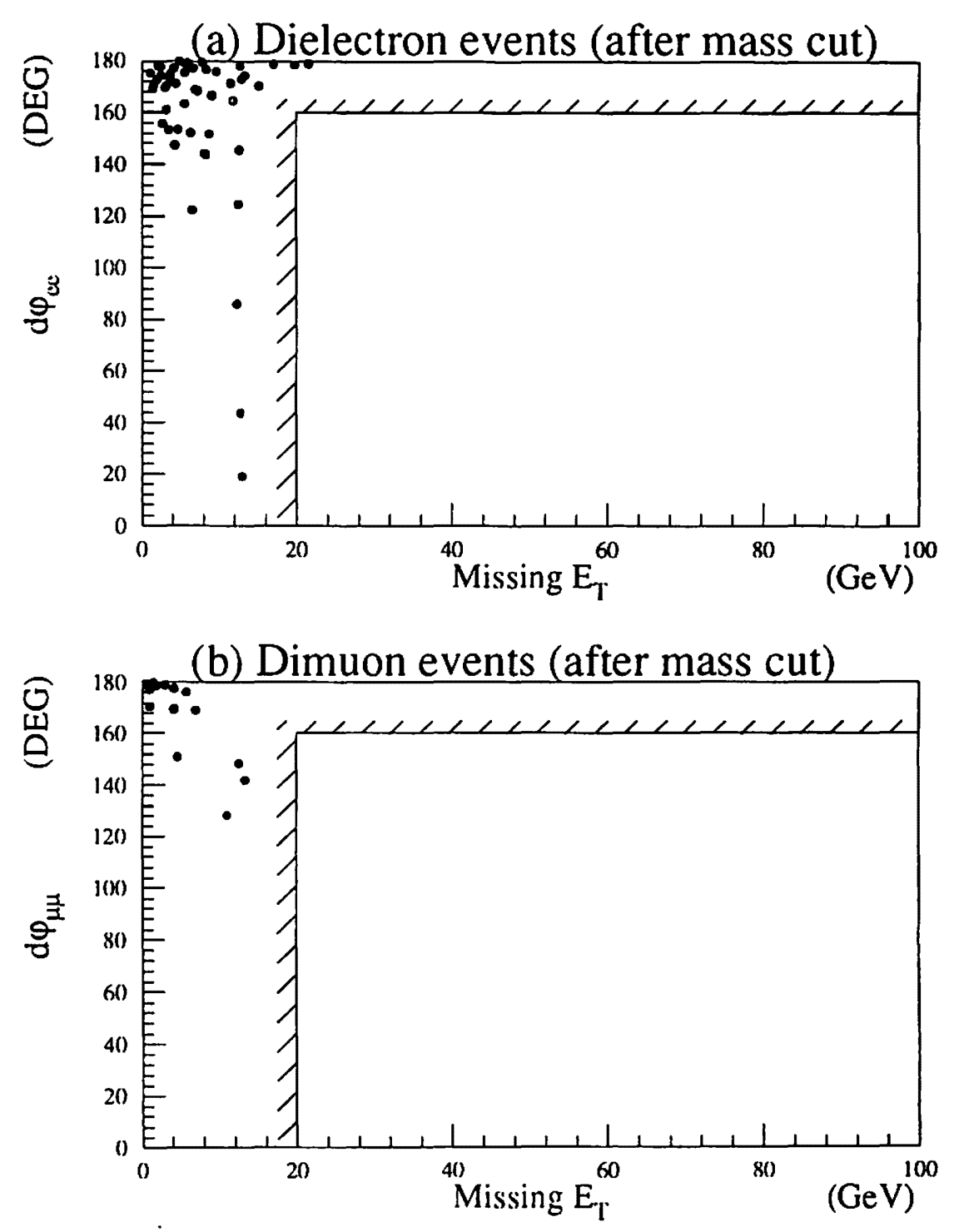

Figure 4.26: The scatter plots of (a) the opening angle between two electrons versus $\mathbb{W}_{\mathrm{T}}$ for the CDF dielectron data and (b) the opening angle between two muons versus $\mathbb{H}_{\mathrm{T}}$ from the CDF dimuon data. 


\section{Chapter 5}

\section{Detection Efficiency and}

\section{Background}

In the previous chapter, the criteria for selecting $t \bar{t}$ events were described and we saw there was only one candidate event found in the CDF data. The production cross section is given by the equation,

$$
\sigma(t \bar{t})=\frac{\left(N-N_{\mathrm{BC}}\right)}{L \cdot \varepsilon_{\text {Total }}},
$$

where $N$ is the number of events passing all the selection cuts, $N_{\mathrm{BG}}$ is the expected number of background events in the final $N$ events, $L$ is the integrated luminosity (4.1 $\mathrm{pb}^{-1}$ in our case) and $\varepsilon_{\text {Total }}$ is the total detection efficiency for the process being searched for. In the present case, an upper limit of the production cross section is extracted without the background subtraction. That is, $\left(N-N_{\mathrm{BG}}\right)$ is replaced by an upper limit of the number of $t \bar{t}$ dilepton events, $N_{\text {max }}$, which is expected to be observed in the data. The $N_{\text {max }}$ is determined based on the fact that there is one event found in the data, with the assumption that this event was a $t \bar{t}$ event. This assumption (no background subtraction) gives a conservative result of the upper limit of the production cross section.

In order to measure the cross section or to give an upper limit of the cross section, it is necessary to know the detection efficiency, $\varepsilon_{\text {Trtal }}$, for the process. In this chapter, the determination of the detection efficiency for $t \bar{t}$ events is described in detail ( $\$ 5.1)$. 
The systematic uncertainty related to the detection efficiency determination is discussed in $\$ 5.2$. In the final section, the background is also estimated although no background subtraction is performed for the final result. This background study shows which background process is severe to the $t \bar{t}$ events. The upper limit of the number of event $N_{\max }$ and the upper limit of the production cross section is calculated in the next chapter. The luminosity measurement is described in Appendix A.

\subsection{Detection Efficiency}

The total detection efficiency $\varepsilon_{\text {Tital }}$ is divided into several terms and written as

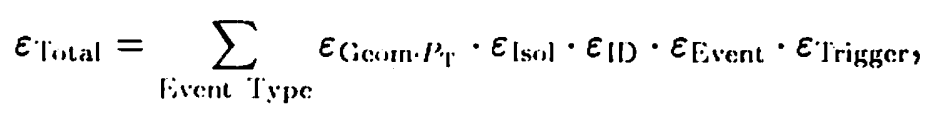

where $\varepsilon_{\text {Germ.Pr }}$ is a combined acceptance of the geometrical cut (the fiducial region) and the kinematical $\left(P_{\mathrm{r}}\right)$ cut, $\varepsilon_{\mid \mathrm{s}, \mathrm{l}}$ is an efficiency of the isolation cut on two leptons in the $t \bar{t}$ events, $\varepsilon_{\mathrm{ID}}$ is an identification cut efficiency for isolated dileptons, $\varepsilon_{\text {Vivent }}$ is an efficiency of the event topology cut for the $t \bar{t}$ events and $\varepsilon_{\text {Trigger }}$ is the trigger efficiency.

The ordering of multiplication has an important meaning. The $\varepsilon_{\text {Isol }}$ is defined as an efficiency for leptons which passed the geometrical and kinematical cuts. As described in $§ 5.1 .1$, both the geometrical and kinematical cuts affect the lepton isolation. The particle activity around leptons vary as $\eta$ changes. It is reflected in the $\eta$ dependence of the isolation cut efficiency. Also high $P_{\uparrow}$ cut tends to select more isolated leptons. Thus the $\varepsilon_{\text {Isol }}$ depends on how the geometrical and $P_{\Gamma}$ cuts were made. Similarly, the $\varepsilon_{\text {ID }}$ is defined as the identification efficiency for leptons in the prescribed geometrical region, which passed both the $P_{T}$ and the isolation cuts. It is obvious that the isolation cut affects the $\varepsilon_{\mid \mathrm{ID}}$ because, for example, the activity of hadrons around an electron might deform the shower shape, resulting in more chances for the electron to fail $\chi^{2}$ test of the lateral shower shape. The $\varepsilon_{\text {licent }}$ is similarly defined for the $t \bar{t}$ events which passed the geometrical cut, the $P_{r}$ cut, the lepton isolation cut and the lepton identification cut. 
Since the lepton reconstruction algorithm in the offline and in the trigger level are different, leptons which passed the offline lepton selection do not ncessarily pass the trigger requirements. For example, due to the different clustering and the different vertex position used in the calculation of the electrons $E_{\mathrm{T}}$, the offline $E_{\mathrm{T}}$ and trigger $E_{\mathrm{T}}$ are different. Therefore we have to measure the trigger efficiency $\varepsilon_{\text {Trigger }}$. The $\varepsilon_{\text {Trigger }}$ has a correlation with the identification cut and the isolation cut because the electron triggers include the $H A D / E M$ cut and the same but tighter cut is applied in the electron identification cut, and because the isolation requirement also changes the $H A D / E M$ cut efficiency which is affected by the hadron activities around an electron. For example, the trigger efficiency for the plug electrons decreases by $\sim 10 \%$ when the isolation and electron identification cuts are removed. The trigger efficiency $\varepsilon_{\text {Trigger }}$ is calculated for leptons which passed the isolation and identification cuts in $§ 5.1 .3$.

The efficiencies, $\varepsilon_{\text {Geom. }} P_{\mathrm{r}}, \varepsilon_{\mathrm{|srl}}$ and $\varepsilon_{\text {Event }}$, are evaluated using Monte Carlo samples because these efficiencies strongly depend on the physics processes. Since there are no $t \bar{t}$ events which allow us to estimate these efficiencies from real data, we have to rely on the Monte Carlo generation and relevant detector simulation to determine them. On the other hand, the efficiencies, $\varepsilon_{||}$and $\varepsilon_{\text {trigger }}$, for high $P_{\Gamma}$ isolated leptons can be measured reliably using real data. We have sufficient $W$ and $Z$ events which provide high $P_{\mathrm{r}}$ isolated leptons for $\varepsilon_{\mathrm{II}}$ estimation. In this case, we do not have to devote much time for detailed tuning of a detector simulation. For the trigger efficiency determination, lepton samples which are collected by the independent triggers are used to see the relation between the offline selection and the trigger selection.

\subsubsection{Monte Carlo Study}

\section{Geometrical and Kinematical Acceptance}

The Monte Carlo $t \bar{t}$ events are generated by the ISAJET generator passed through the CDF detector simulation (CDFSIM), and finally reconstructed using the same codes as for the real CDF data. The reconstructed lepton candidates include real leptons 
originating from top quarks and also real or fake leptons from other sources such as from hadronization and decays of partons in the initial or final parton showers. "Reconstructed lepton" means a lepton candidate at the reconstruction stage. For electrons, the requirements of $E_{\top}>5 \mathrm{GeV}$ and $H A D / E M<0.125$ are imposed at this stage. For muons, all the CTC tracks are considered as muon candidates and no other cut is required explicitly.

Since we deal with real leptons from top quark decays, it is necessary to relate leptons reconstructed by the CDF offline codes to real leptons from top quarks. First, leptons which originate from $t / \bar{t}$ are searched at the event generator level. We examine the matching in $\eta$ - $\phi$ space between these GENerator Particles (GENP) and the reconstructed lepton candidates, taking $p \bar{p}$ collision vertex smearing into account. Figure 5.1 shows the distributions of the distance between the GENP leptons from top quarks with lepton $P_{\mathrm{T}}>15 \mathrm{GeV} / c$ and the nearest reconstructed lepton candidates. This matching is required to be less than 0.1 for electrons and 0.04 for muons. Leptons which passed the matching cut are considered as the ones from $t / \bar{t}$ and successfully reconstructed by the CDF detector. Figure 5.2 shows the efficiency for the GENP leptons to have a matched reconstructed lepton candidate, or equivalently, the efficiency for leptons to be roughly recognized in the detector, as a function of lepton $P_{\mathrm{T}}$. It is very efficient over $P_{\mathrm{T}}=15$ $\mathrm{GeV} / c$. The efficiencies for GENP leptons with $P_{\Upsilon}>15 \mathrm{GeV} / c$ are $\sim 90 \%$ for electrons and $\sim 99 \%$ for muons. The inefficiency for electrons is attributed to a $H A D / E M$ cut explicitly required at the reconstruction stage.

For reconstructed leptons which passed the kinematical cut and the matching test with GENP leptons from top quarks, the fiducial cut efficiencies are summarized in Table 5.1. The geometrical coverage of the fiducial region for the electron is $80 \%$ of the solid angle for the central region $(|\eta| \leq 1.1)$ and $60 \%$ for the plug region $(1.1 \leq|\eta| \leq 2.4)$. The geometrical distribution of electrons in the $t \bar{t}$ events changes these numbers to those in the table. The track quality cut is included in the cut efficiency for muons. Note that only the track quality cut and no explicit fiducial cut is applied for MUs. Adding up 


\begin{tabular}{|c|c|c|c|c|}
\hline & CE & PE & MU & MI \\
\hline$\varepsilon(\%)$ & $84.5 \pm 0.4$ & $70.3 \pm 0.9$ & $>99$ & $65.5 \pm 0.7$ \\
\hline
\end{tabular}

Table 5.1: The fiducial cut efficiencies for each type of leptons. For muons, the track quality cuts are included. The ISAJET Monte Carlo with $M_{\text {top }}=90 \mathrm{GeV} / c^{2}$ was used to obtain the efficiencies.

the number of dilepton pairs over all 8 dilepton classes, the fiducial cut efficiency for the dilepton $t \bar{t}$ events is obtained to be about $70 \%$. The combined efficiency $\varepsilon_{\mathrm{G} c o m} \cdot P_{\mathrm{T}}$ of the matching cut, the kinematical cut and the fiducial cut for each dilepton class is summarized in the second column of Table 5.6 for $M_{\mathrm{Lop}}=90 \mathrm{GeV} / c^{2}$ where the $\varepsilon_{\mathrm{Gcom}} \cdot P_{\mathrm{T}}$ is normalized to the total number of $t \bar{t}$ events times the nominal dilepton branching fraction of $4 / 81$.

\section{Isolation}

The lepton isolation depends on the $\eta$ and $E_{1}$ of the lepton. In order to see the $\eta$ and $E_{\mathrm{T}}$ dependence of the isolation cut efficiency, the isolation variable is calculated at the GENP level. It is defined in a similar way as the one used in the central electron selection, that is, the total $E_{\text {T }}$ of particles except $\nu$ 's within the cone radius of 0.4 around a lepton. Figure 5.3 shows the isolation cut efficiency as a function of $\eta$ for GENP electrons with $P_{\mathrm{T}}>5 \mathrm{GeV}$. We see the cut efficiency increases as $\eta$ increases becuase there are less high $P_{\mathrm{T}}$ particles in large $\eta$ region. Figure 5.4 shows the cut efficiency as a function of $E_{\mathrm{T}}$ for GENP central electrons. In the same geometrical area, higher $E_{\mathrm{T}}$ leptons tend to be well isolated as we expect. Considering these dependences, the isolation cut efficiencies are defined for each type of leptons (CE, PE, MU, MI) which passed the geometrical cut and kinematical cut.

As a check of the detector simulation, we have compared the isolation distributions for leptons from $Z$ decays in the CDF data with Monte Carlo predictions (see Figure 5.5). The small energy detected around leptons from $Z$ decays comes mostly from the underlying event, or fragments from the spectator partons. Figure 5.6 shows a compar- 


\begin{tabular}{|c|c|c|c|c|}
\hline$M_{\text {top }}\left(\mathrm{GeV} / c^{2}\right)$ & $\begin{array}{c}\mathrm{CE} \\
\%\end{array}$ & $\begin{array}{c}\mathrm{PE} \\
\%\end{array}$ & $\begin{array}{c}\text { MU } \\
\%\end{array}$ & $\begin{array}{c}\text { MI } \\
\%\end{array}$ \\
\hline 70 & $88 \pm 4$ & $79 \pm 4$ & $78 \pm 4$ & $82 \pm 4$ \\
80 & $88 \pm 4$ & $85 \pm 4$ & $81 \pm 4$ & $82 \pm 4$ \\
90 & $88 \pm 4$ & $87 \pm 4$ & $81 \pm 4$ & $85 \pm 4$ \\
100 & $84 \pm 4$ & $84 \pm 4$ & $76 \pm 4$ & $81 \pm 4$ \\
\hline
\end{tabular}

Table 5.2: The isolation cut efficiencies for each type of leptons from top quark of several masses.

ison between low $P_{\mathrm{T}}$ e $\mu$ events, consisting predominantly of $b \bar{b}$ dilepton events, and $b \bar{b}$ Monte Carlo predictions. In this case, energy flow around leptons includes particles from initial and final gluon radiations. There is a good agreement in both cases, which gives a confidence in the detector simulation and the Monte Carlo modeling of the underlying event and the gluon radiations. Leptons from top quarks with $M_{\mathrm{top}}=90 \mathrm{GeV} / c^{2}$ are much isolated than the ones from bottom quarks but not so much as the ones in $Z$ decays as shown in Figure 5.7.

The isolation cut efficiencies for top leptons, obtained from ISAJET + CDFSIM Monte Carlo data, are shown in Table 5.2. The isolation cut efficiency for a specific event type, $\varepsilon_{\mathrm{ls} \text { ol }}$, is a product of the two efficiencies for each lepton.

The isolation cut efficiency changes with the top quark mass. The efficiency gets better as $M_{\text {top }}$ becomes large and approaches the $W$ mass, as expected. Around the $W$ mass region, $b$ quark from $t \rightarrow W b$ decay becomes soft and may not be reconstructed as a jet, because most of the top quark mass energy is carried by the $W$. Beyond the $W$ mass region where a $b$ quark starts to be reconstructed as a jet, leptons from $W \mathbf{s}$ produced in $t \rightarrow W b, W \rightarrow \ell \nu$ get overlapped with the $b$ jets. Thus the isolation cut efficiency is degraded slightly.

\section{Event Topology Cuts}

The efficiency of the event topology cut $\varepsilon_{\text {licin }}$ for each event type calculated from the ISAJET+CDFSIM Monte Carlo with $M_{\text {(rip }}=90 \mathrm{GeV} / c^{2}$ is summarized in Table 5.6. 


\subsubsection{Lepton Identification Efficiency}

\section{Central Electron}

The efficiencies of the central electron identification criteria are determined from a sample of $Z \rightarrow e e$ events. Let $N$ be the total number of $Z \rightarrow e e$ events produced. The $N$ can not be directly counted since the efficiency $\varepsilon$ for identifying an electron is not a priori known. Call $N_{1}$ the number of $Z \mathrm{~s}$ for which at least one leg passes the identification cut, and $N_{2}$ the number of $Z \mathrm{~s}$ for which both legs pass the cut. We write $\varepsilon_{c}$ for the efficiency that the electron passes a single cut $c$ in several identification cuts, and call $N_{c}$ the number of $Z$ s where one leg passes all the cuts and the other leg passes cut $c$. The observed numbers $N_{1}, N_{2}$ and $N_{c}$ are related to the unknown numbers, $N, \varepsilon$ and $\varepsilon_{c}$ by

$$
\begin{aligned}
& N_{1}=\varepsilon(2-\varepsilon) N, \\
& N_{2}=\varepsilon^{2} N, \\
& N_{c}=\varepsilon\left(2 \varepsilon_{c}-\varepsilon\right) N .
\end{aligned}
$$

From these equations we get,

$$
\begin{aligned}
N & =\frac{\left(N_{1}+N_{2}\right)^{2}}{4 N_{2}}, \\
\varepsilon & =\frac{2 N_{2}}{N_{1}+N_{2}}, \\
\varepsilon_{\mathrm{r}:} & =\frac{N_{2}+N_{c}}{N_{1}+N_{2}}
\end{aligned}
$$

The $Z \rightarrow e e$ events used for this study were selected by requiring one electron candidate passing the strict selection requirements and a second isolated EM cluster which forms an invariant mass with the electron between 80 and $105 \mathrm{GeV} / \mathrm{c}^{2}$.

The central electron identification efficiencies for each cut and the combined cut are summarized in Table 5.3. The total identification efficiency is found to be 


\begin{tabular}{|c|c|c|}
\hline Tight Cuts & $\varepsilon($ CDF $Z)$ & $\varepsilon$ (Monte Carlo $Z)$ \\
\hline$H A D / E M<0.05$ & $0.97 \pm 0.01$ & $0.98 \pm 0.01$ \\
$L_{\text {shr }}<0.2$ & $0.98 \pm 0.01$ & $0.97 \pm 0.01$ \\
$\chi_{\text {strip }}^{2}<10$ & $0.95 \pm 0.02$ & $0.87 \pm 0.02$ \\
$E / P<2$ & $0.96 \pm 0.01$ & $0.99 \pm 0.01$ \\
$D_{\mathrm{X}}<1.5 \mathrm{~cm}$ & $0.98 \pm 0.01$ & $0.99 \pm 0.01$ \\
$D_{\mathrm{Z}}<3.0 \mathrm{~cm}$ & $0.99 \pm 0.01$ & $0.96 \pm 0.01$ \\
\hline Total & $0.88 \pm 0.03$ & $0.78 \pm 0.02$ \\
\hline \hline Loose Cuts & $\varepsilon$ (CDF $Z)$ & $\varepsilon$ (Monte Carlo $Z)$ \\
\hline$H A D / E M<0.055+0.045 E_{\mathrm{T}}(e) / 100$ & $0.98 \pm 0.02$ & 1. \\
\hline Total & $0.98 \pm 0.02$ & 1. \\
\hline
\end{tabular}

Table 5.3: The central electron identification cut efficiencies calculated from the CDF $Z \rightarrow e e$ and Monte Carlo $Z \rightarrow e e$ events.

$$
\begin{aligned}
& \varepsilon_{\mathrm{ID}}(\mathrm{CE} ; \text { Tight })=(88 \pm 3) \%, \\
& \varepsilon_{\mathrm{ID}}(\mathrm{CE} ; \text { Loose })=(98 \pm 2) \% .
\end{aligned}
$$

It is interesting to know how well the CDF detector simulation, CDFSIM, models the actual detector. Figures 5.8 and 5.9 show distributions of the lepton identification variables, in comparison between real $Z \rightarrow e e$ events and the CDFSIM simulation. The efficiencies calculated from the Monte Carlo sample are also shown in Table 5.3. The total efficiency does not agree completely with the measured efficiency. The main source is a discrepancy in the strip shower profile cut between the real data and the Monte Carlo, which has been recognized as a problem of the CDFSIM.

Since the identification cut efficiency $\varepsilon_{\| 1}$ has an isolation dependence as shown in Figure 5.10, the obtained efficiency from $Z \rightarrow e e$ is valid only for electrons with $E_{\mathrm{T}}^{\text {conc }}<5$ $\mathrm{GeV}$. It was checked using Monte Carlo data whether the identification cut efficiency for high $P_{\text {r }}$ isolated electrons from $Z \rightarrow e e$ events and from $t \bar{t}$ events agrees with each other. The ratio of the two efficiencies is found to be $97 \pm 3 \%$ indicating that the identification cut efficiency obtained from real $Z \rightarrow e e$ events is also valid for high $P_{\mathrm{T}}$ isolated electrons in $t \bar{t}$ events. 
Some of the non-"conversion" (prompt) electrons are wrongly identified as "conversion" electrons by the algorithm described in $\S 4.3 .1$. Since the cut on the VTPC hit occupancy $f_{\text {VTPC }}>0.2$ is found to be quite high efficient for real electrons by looking at the $Z \rightarrow e e$ events, the over-efficiency of the conversion identification algorithm comes from the cut $m_{c e}>0.5 \mathrm{GeV}$. Namely, one of the uncorrelated tracks accidentally forms a low invariant mass with the electron candidate. Since the manner how uncorrelated tracks distribute around the electron candidate is expected to be same for like-sign tracks and unlike-sign tracks to the candidate electron, one can estimate the inefficiency of the conversion removal for prompt electrons by looking at $m_{c e}$ which is formed by a same sign charged track with the electron candidate. To this aim, central electrons which pass all the selection cuts except the conversion removal were collected. Let us denote by $N$ the number of electrons of that sample passing $f_{V \mathrm{TPC}}>0.2$. Then the inefficiency of conversion identification algorithm for prompt electrons are given by

$$
f_{\text {prompt }}=\frac{N^{\prime}}{N}
$$

The $N^{\prime}$ is the number of electron candidates which pass the cut $f_{V T P C}>0.2$ and $m_{e e}<0.5 \mathrm{GeV} / c^{2}$ where $m_{e c}$ is calculated with a like-sign track to the candidate electron. This method gave

$$
f_{\text {primint }} \sim 5 \%
$$

Since this over-efficiency depends on the charged particle multiplicity around the prompt electron candidate, it would change from physics process to process being studied. Thus $f_{\text {prompt }}$ was calculated for $t \bar{t}$ Monte Carlo data. It was found to be $3 \%$ for $M_{\text {top }}=90$ $\mathrm{GeV} / c^{2}$. This overkilling efficiency (0.03) of the conversion removal for central electrons is multiplied to the electron identification efficiency in the calculation of $\varepsilon_{\text {ID }}$.

\section{Plug Electron}

The efficiencies of the plug electron identification cuts are determined using real $Z \rightarrow e e$ 
events. The $Z$ sample is selected by requiring one central electron satisfying the strict cuts and an isolated plug EM cluster which forms an invariant mass with the central electron between 80 and $100 \mathrm{GeV} / c^{2}$. This selection results in 89 events. The total efficiency is found to be

$$
\varepsilon_{\mathrm{ID}}(\mathrm{PE})=(79 \pm 4) \%
$$

A statistically larger sample of $W \rightarrow e \nu$ events can also be used to determine the efficiency as a check. We select 771 events with one isolated plug EM cluster, $\not_{\mathrm{T}}>30$ $\mathrm{GeV}$ and no additional calorimeter energy cluster with $E_{\mathrm{T}}>5 \mathrm{GeV}$. The number of plug electron candidates passing all the identification cuts is 406 , resulting in an apparent efficiency of $53 \%$ which is significantly lower than the efficiency determined from $Z$ decays. This low efficiency is attributed to a background contamination in the $W$ sample. To determine the efficiency from the $W$ sample, further requirements are needed to minimize the effects of the background contamination. From a study on $Z \rightarrow e e$ sample, it was found that there was no signification correlation between a CTC track matching cut in $\phi$ and other cuts, $H A D / E M, \chi_{3 \times 3}^{2}$ and VTPC hit occupancy cut. Hence the CTC $\phi$ matching is required when estimating the combined efficiency of the $\chi_{3 \times 3}^{2}, H A D / E M$ and $f_{V T P C}$ cuts. The track matching requirement removes $\pi^{0} / \gamma+\pi^{ \pm}$overlaps and reduces the background by a factor of approximately two. Then, by first applying the $\chi_{3 \times 3}^{2}, H A D / E M$ and $f_{V T P C}$ cuts, we determine the efficiency of the track matching requirement. The total efficiency is calculated as a product of these two efficiencies. As a check, this method gives $(81 \pm 5) \%$ efficiency for $Z$ s which shows a good agreement with the previous result indicating the observation of no strong correlation between the CTC $\phi$ matching and others cuts. For electrons in the $W$ sample, the efficiency was changed to $(79 \pm 2) \%$ with this method which is in good agreement with the results from the $Z \rightarrow e e$ study.

Since the plug region is not completely covered by the CTC, the tracking information is limited. The reliable momentum measurement is possible only for $|\eta|<1.7$. And the performance of the CTC track reconstruction becomes poor rapidly as $|\eta|$ increases. 


\begin{tabular}{|c|c|c|}
\hline Cuts & $\varepsilon($ CDF $Z)$ & $\varepsilon$ (Monte Carlo Z) \\
\hline$H A D / E M<0.1$ & $>0.99$ & $>0.99$ \\
$\chi_{3 \times 3}^{2}<10$ & $0.96 \pm 0.03$ & $0.91 \pm 0.02$ \\
$f_{\text {VTPC }}>0.7$ & $0.94 \pm 0.02$ & $0.95 \pm 0.01$ \\
Track matching & $0.84 \pm 0.04$ & $0.85 \pm 0.04$ \\
\hline Total & $0.79 \pm 0.04$ & $0.72 \pm 0.02$ \\
\hline
\end{tabular}

Table 5.4: The plug electron identification cut efficiencies calculated from CDF $Z \rightarrow e e$ and Monte Carlo $Z \rightarrow$ ee events.

On the other hand, the VTPC covers the region beyond the CTC. Contrary to the CTC, the VTPC tracking performance gets better in larger $|\eta|$ region because reliable $\phi$ measurements are possible in this region where particles traverse more VTPC modules than those in the small $|\eta|$ region (See Figure 5.11). Since we would like to use the efficiency measured from $Z s$, it is desirable that the efficiency is independent of $\eta$ in view of the different $\eta$ distributions of $Z$ and top electrons. Considering the different tracking performance of the CTC and the VTPC along $\eta$, we make a logical OR of the CTC and VTPC matching. The combination of the two has only a weak rapidity dependence (See Fig. 5.12) and keeps the efficiency high over the full plug $\eta$ region.

The efficiencies for the plug electron identification are listed in Table 5.4. The efficiencies obtained from ISAJET + CDFSIM $Z$ Monte Carlo are also shown in the table for comparison. There is a small discrepancy in the $\chi_{3 \times 3}^{2}$ efficiencies, which is deeply related to the correctness of the modeling of the shower shape. The CDFSIM must be tuned in this respect.

\section{Rejection Power of Electron Identification Against Backgrounds}

It is important to know not only the identification efficiency for electrons but also the rejection power against backgrounds. It will also tell us which tool is effective for the electron identification. For this study, $\gamma$ plus jets events are used. These events consist of high $E_{\mathrm{T}} \gamma$ with a recoiling jet back to the $\gamma$. Since there is no expected source for $\gamma$ plus a recoiling real electron, the EM clusters back to the $\gamma$ 's are dominated by the 
backgrounds.

The EM clusters are first required to pass $E_{\mathrm{T}}>15 \mathrm{GeV}$ and some very loose track matching to reject $\gamma$ 's. The reduction factors of these EM clusters when each electron identification cut is applied is shown in Figure 5.13 both for the central and the plug EM clusters together with the efficiencies for real electrons to pass the cut measured from $Z$ events. The $H A D / E M$ cut which is expected to be the most effective in removing charged pions shows only a weak power because these EM clusters have already satisfied $H A D / E M<0.125$ imposed at the reconstruction stage. We see the most effective identification tool for the central electron is the one using tracking information. Although the track matching requirement in the plug electron identification reduces background by a factor of about two, it is worse than that for the central electrons both in the efficiency and the reduction power. However, the disadvantage in the tracking tool for the plug electron identification is greatly compensated by the other requirements such as the higher $E_{\gamma}$ cut or the isolation requirement.

The event kinematical and topological constraints are also effective for obtaining better quality of a sample. For example, the event $W \rightarrow e \nu$ is well identified by the presence of a large missing $E_{\uparrow}$ and the $Z \rightarrow e e$ event is well identified by requiring the presence of one more good lepton in an event. On the other hand, study of $c \bar{c}$ or $b \bar{b}$ production where electrons have more moderate $E_{\mathrm{T}}$ and are not isolated is difficult without a help of the tracking.

\section{Central Muon}

The efficiencies for the central muons (actually for one of two muon classes, CMUOs) are measured using the $Z \rightarrow \mu \mu$ events in a similar way as in the central electron identification efficiency determination. The results are summarized in Table 5.5. Since the position matching cut $D_{X}^{\prime \prime}$ is quite high efficient, the identification cut efficiency for CMUOs is almost equal to the efficiency for CMIOs which are required only a minimum ionizing cut. We conclude that the muon identification efficiency including both CMUO 


\begin{tabular}{|c|c|c|}
\hline Cuts & $\varepsilon($ CDF Z) & $\varepsilon$ (Monte Carlo Z) \\
\hline$D_{x}^{\mu}<10$. & $>0.99$ & $>0.99$ \\
Minimum ionizing & $0.98 \pm 0.01$ & $0.98 \pm 0.01$ \\
\hline Total & $0.98 \pm 0.01$ & $0.98 \pm 0.01$ \\
\hline
\end{tabular}

Table 5.5: The muon identification cut efficiencies calculated from CDF $Z \rightarrow \mu \mu$ and Monte Carlo $Z \rightarrow \mu \mu$ events.

and CMIO type of muons is

$$
\varepsilon_{\mathrm{II})}(\mu)=(98 \pm 1) \%
$$

The lepton identification efficiency $\varepsilon_{I I)}$ for a specific event type is a product of the two efficiencies for each lepton.

\subsubsection{Trigger Efficiency}

\section{Central Electron Trigger}

In order to estimate the trigger efficiency, we need an unbiased central electron sample, that is, the events which contain central electrons but are accepted by at least one of the triggers independent of the central electron $12-\mathrm{GeV}$ trigger. As such samples, the electron sample collected by the central electron trigger with a lower threshold of 7 $\mathrm{GeV}$, and $Z \rightarrow e e$ events collected by the dielectron trigger with an $E_{\mathrm{T}}$ threshold of 5 $\mathrm{GeV}$ on both electrons were used. The efficiency that the isolated electron candidates which passed the identification cut satisfy the requirements of the $12-\mathrm{GeV}$ trigger was obtained as a function of the offline $E_{\Gamma}$. Figure 5.14 (a) shows this trigger efficiency curve $[20,19,63]$. With the $12-\mathrm{GeV}$ trigger $E_{\mathrm{T}}$ threshold, the trigger efficiency for isolated central electrons with $E_{\Upsilon}>15 \mathrm{GeV}$ was determined to be

$$
\varepsilon_{\text {Jrigger }}(\mathrm{CE})=(98.0 \pm 0.5) \%
$$




\section{Plug Electron Trigger}

In order to estimate the efficiency of the plug electron trigger, an inclusive central and plug electron sample where electrons were required to pass the identification cut was used [64]. Events which have EM clusters in the plug region and triggered independently of the plug electron trigger were selected. To get more statistical power, events which hit only the plug electron trigger were also collected if there were additional clusters found in the plug. At this time, plug EM clusters were required to pass the fiducial cut, isolation cut and some of the identification cuts. Comparing the offline $E_{\mathrm{T}}$ of these clusters with the corresponding level-2 trigger $E_{\uparrow}$, the efficiency as a function of the offline $E_{\mathrm{T}}$ was obtained, as shown in Figure 5.14 (b). The rather slow turning-on of the trigger is due to the several effects:

- the trigger $E_{\mathrm{T}}$ calculation assumed that the interaction occurred at $Z_{\text {event }}=0$ while the offline $E_{T}$ used a measured event vertex. The $Z_{\text {cren }}$ distribution had a standard deviation of $35 \mathrm{~cm}$.

- the trigger used the energy information from one of the three depth segments of the PEM.

- there was a gain variation in the PEM quadrants which was corrected in the offline analysis but which was not considered in the trigger.

- there were a small number of non-functioning chambers in the PEM which caused a degradation of the calorimeter response which was also not accounted for in the trigger.

The efficiency of the plug electron $23-\mathrm{GeV}$ trigger was $(50 \pm 6) \%$ at $E_{\mathrm{T}}=30 \mathrm{GeV}$. Using this trigger efficiency curve and the $E_{\mathrm{T}}$ distribution for plug electrons from top Monte Carlo with $M_{\mathrm{Lap}}=90 \mathrm{GeV} / c^{2}$, we get an efficiency of $(49 \pm 3) \%$ for electrons 
with $E_{\mathrm{T}}>15 \mathrm{GeV}$ and $(85 \pm 3) \%$ for electrons with $E_{\mathrm{T}}>30 \mathrm{GeV}$.

\section{Central Muon Trigger}

The combined level-1 and level-2 trigger efficiency for central muons with $P_{\mathrm{T}}>15 \mathrm{GeV} / c$ was determined in a similar way [65]. A cosmic ray data which was taken in a special CDF run, and the isolated muon sample which was collected by an independent trigger were used. The obtained efficiency is

$$
\varepsilon_{\text {Trigger }}(\mathrm{MU})=(91 \pm 2) \%
$$

The trigger efficiency as a function of the offline $P_{\gamma}$ is shown in Figure 5.14 (c). The inefficiency was found to be dominated by the level-1 inefficiency, which was caused mostly by delta rays that affected the angle measurement in the muon chambers. Because of a hardware malfunction during the early part of the run, only $3.5 \mathrm{pb}^{-1}$ of data were collected with the muon trigger.

The trigger efficiency for a specific event type, $\varepsilon_{\text {lrigger }}$, is calculated using the trigger efficiencies for the single lepton triggers determined above. An additional correction is applied to the efficiency of the central muon trigger which was not operational during the first $15 \%$ of data taking. Since there are two leptons in our events, the trigger efficiency for a specific event type, $\varepsilon_{\text {Triggcr, }}$, is calculated as $1-f_{1} \cdot f_{2}$ where $f_{1}$ and $f_{2}$ are the separate probabilities for failing the first and second lepton triggers, respectively. If there is no trigger for one of the two leptons, we have $f=1$ and the $\varepsilon$ 'rigger is equal to the single lepton trigger efficiency.

\subsubsection{Summary}

The various cut efficiencies are listed for each of the dilepton event types in Table 5.6 for $M_{\text {top }}=90 \mathrm{GeV} / c^{2}$. The total dilepton efficiency at this mass is $\varepsilon_{\text {Total }}=(16.2 \pm 1.8) \%$, 


\begin{tabular}{|ccccccc|}
\hline Event class & $\begin{array}{c}\varepsilon_{\text {Geom.'P }} \\
\%\end{array}$ & $\begin{array}{c}\varepsilon_{\text {Isol }} \\
\%\end{array}$ & $\begin{array}{c}\varepsilon_{|l|} \\
\%\end{array}$ & $\begin{array}{c}\varepsilon_{\text {Livent }} \\
\%\end{array}$ & $\begin{array}{c}\varepsilon_{\text {Trigger }} \\
\%\end{array}$ & $\begin{array}{c}\varepsilon_{\text {Total }} \\
\%\end{array}$ \\
\hline \hline CE-CE & 6.7 & 77 & 89 & 61 & 100 & 2.9 \\
CE-MU & 9.2 & 71 & 84 & 85 & 100 & 4.6 \\
CE-MI & 7.5 & 74 & 84 & 83 & 98 & 3.8 \\
CE-PE & 3.8 & 76 & 67 & 53 & 99 & 1.0 \\
MU-MU & 3.1 & 65 & 96 & 70 & 85 & 1.2 \\
MU-MI & 4.6 & 68 & 96 & 58 & 78 & 1.4 \\
MU-PE & 2.5 & 70 & 77 & 91 & 81 & 1.1 \\
PE-MI & 1.0 & 74 & 77 & 90 & 85 & 0.42 \\
\hline Total & 38.3 & 72.4 & 84.7 & 73.0 & 94.6 & $16.2 \pm 1.8$ \\
\hline
\end{tabular}

Table 5.6: Dilepton detection efficiencies for $M_{\text {(op) }}=90 \mathrm{GeV} / c^{2}$. The total dilepton efficiency at this mass is $\varepsilon_{\text {rital }}=(16.2 \pm 1.8) \%$. The efficiency $\varepsilon_{\mathrm{Gcom}} \cdot P_{\mathrm{T}}$ is relative to the nominal dilepton branching fraction (4/81) of $t \bar{t}$ events.

corresponding to $0.162 \times 4 / 81=0.80 \%$ of the $t \bar{t}$ cross section. The uncertainty in $\varepsilon_{\text {Total }}$ is discussed in $§ 5.2$. The small top quark mass dependence of the efficiencies is illustrated in Figure 5.15.

\subsection{Systematic Uncertainty}

The total detection efficiency for dileptons from $t \bar{t}$ events, $\varepsilon_{\text {Total }}$, is affected by systematic uncertainties associated with each of factor on the right hand side of Equation (5.2). In this section, the systematic uncertainties in the detection efficiency determination are discussed. The numbers are given in terms of the fractional uncertainties or the relative ratios of absolute uncertainty to overall total detection efficiency $\left(\Delta \varepsilon_{\text {Total }} / \varepsilon_{\text {Total }}\right)$.

\section{Geometrical and Kinematical Acceptance}

The systematic uncertainty in the geometrical and kinematical acceptance was estimated to be $4 \%$ by observing a variation in the acceptance when the PAPAGENO Monte Carlo [66] was used instead of the ISAJET. As a check, the uncertainty is also estimated by 
changing the combinations of the structure functions and the definition of the scale parameter $\mu$. The structure functions used were EHLQ, MRS and DFLM. The $\mu$ definitions varied include $P_{\mathrm{T}}^{2} / 4, P_{\mathrm{T}}^{2}, 2 \hat{s} \hat{t} \hat{u} /\left(\hat{s}^{2}+\hat{t}^{2}+\hat{u}^{2}\right)$ and $\hat{s}$ where $P_{\mathrm{T}}$ is the transverse momentum of the top quark and $\hat{s}, \hat{t}, \hat{u}$ are the standard Mandelstam invariants. The mass of the top quark was set to $100 \mathrm{GeV} / \mathrm{c}^{2}$. We tried all possible combinations and counted the number of events which passed the required cuts on acceptance. The event vertex was smeared but no smearing due to the detector resolution was done on $E_{\mathrm{T}}$ nor $P_{\mathrm{T}}$. The systematic uncertainty was taken as half of a span between the two extreme values in the combinations. For the central dilepton only, the uncertainty is $8 \%$ and the inclusion of plug reduces it to $5 \%$.

\section{Lepton Isolation Cut}

Several sources of the uncertainty in the lepton isolation cut efficiency include:

- modeling of the detector simulation

- gluon radiation

- fragments from spectator partons (called "beam jets" or "underlying event")

- fragmentation of $t$ and $b$ quarks.

Generally particle activities around a lepton can be classified into 3 groups, particles originating from the hard scattering of partons, particles from the initial state radiation of hard colliding partons, and particles from interactions of the spectator partons. The energy flow from the hard scattering can be further classified into the one from top fragmentation (the extra particles $X$ in $t \rightarrow T+X$ where $T$ is a $t$ hadron), the one from $t$ hadron decay, and the one from the final state gluon radiation emitted off the $t$ quark. Figures 5.16 and 5.17 show contributions of each process to the GENP isolation of the central electron with $E_{\mathrm{r}}>15 \mathrm{GeV}$ from $t \bar{t}$ Monte Carlo with $M_{\text {(op) }}=90 \mathrm{GeV} / c^{2}$. The particle activities from the hard scattering process dominates around the lepton $(\sim 75 \%)$. 
Since the heavier the quark mass becomes, the fragments except the heavy hadron are softer (harder fragmentation), the particle activities from the top quark fragmentation is relatively small as shown in Figure 5.17. The ambiguity in the fragmentation is, therefore, expected to be small. Similarly the uncertainty from the underlying event is considered to be small because of the small contribution from this process to the isolation of leptons. On the other hand, the non-negligible contribution from the initial state radiation $(\sim 15 \%)$ is observed in Figure 5.16 resulting in one of main uncertainties of the isolation cut efficiency as described below.

The difference of the isolation efficiencies for GENP leptons and for reconstructed leptons is found to be less than $8 \%$. A half of it $4 \%$ could be considered as the uncertainty related to the calorimeter simulation. The distributions of isolation variables show, however, good agreement between CDF data and Monte Carlo, both for $Z$ events and $b \bar{b}$ events as we saw before in $§ 5.1 .1$. Hence the uncertainty due to the detector simulation is something between $0-4 \%$. We take $2 \%$. The uncertainty from the fragmentation was estimated by varying the Peterson parameter [26] in the range of $0.2 / M_{\text {top }}^{2}-1.5 / M_{\text {Lop }}^{2}$. The change in the efficiency was $\sim 1 \%$. The uncertainty induced by the bottom quark fragmentation turned out to be negligible. The uncertainty due to the modeling of the gluon radiation was estimated to be $3 \%$ by comparing the efficiency change due to turning on/off of the radiation in the ISAJET Monte Carlo. Finally the uncertainty from the underlying event was estimated to be $1 \%$ by toggling on/off the beam jets in the ISAJET. Adding these uncertainties due to the several sources in quadrature, we have $\sim 4 \%$ fractional uncertainty $(\Delta \varepsilon / \varepsilon)$ in the isolation cut efficiency for a single lepton. Sine the isolation cut efficiency for a specific event class $\varepsilon_{\text {Isol }}$ is given as a product of the cut efficiencies for two leptons, a resulting fractional uncertainty is estimated to be $8 \%$. Note that this estimate is very conservative because it is based on the comparison of the extreme cases, turning on/off of the radiation for instance, while the agreement between data and Monte Carlo indicates the modeling related to the isolation quantity is working well. 


\section{Others}

Lepton identification efficiencies were estimated using CDF data as described before in $\S 5.1 .2$. Their statistical uncertainties are propagated to an uncertainty in $\varepsilon_{\text {Total }}$ of $5 \%$.

The uncertainty in the $\mathbb{W}_{\mathrm{T}}$ cut efficiency is closely related to how well the Monte Carlo models actual events and detector responses. For the top quark mass region being probed, the $b$ jets are soft, and we expect the uncertainty in $E_{\tau}$ measurement to be similar for $t \bar{t}$ events and for $W$ events. A very good agreement in $\mathbb{E}_{\mathrm{T}}$ distributions between Monte Carlo and $W$ data (see Figure 5.18) indicates the uncertainty in the $\mathbb{W}_{\mathrm{T}}$ cut efficiency for the $t \bar{t}$ dilepton events is small. Uncertainty of $10 \%(20 \%)$ in $\mathbb{W}_{\mathrm{T}}$ measurement results in $1 \%(2 \%)$ overall uncertainty in $\varepsilon_{\text {rotal }}$ for $M_{\text {Lop }}=100 \mathrm{GeV} / c^{2}$.

The statistical uncertainties in the trigger efficiency measurements are propagated to an uncertainty of $\Delta \varepsilon_{\text {Total }} / \varepsilon_{\text {Tital }} \simeq 2 \%$.

Since the geometrical and kinematical acceptance, the isolation cut efficiency and the event topology cut efficiency are obtained using the same Monte Carlo sample, there is a correlation in the statistical fluctuations among them. Therefore the Monte Carlo statistical uncertainty was assigned to the combined efficiency of these three factors. The number of the total generated events is about $180 \mathrm{~K}$ for each top quark mass (70, 80,90 , and $\left.100 \mathrm{GeV} / \mathrm{c}^{2}\right)$. The statistical uncertainty in the combined efficiency is $\sim 2 \%$ for all the masses being probed.

\section{Summary}

Adding these uncertainties in quadrature gives a total uncertainty of

$$
\Delta \varepsilon_{\text {Trital }} / \varepsilon_{\text {Trial }} \simeq 11 \% \text {. }
$$

Adding further the uncertainty of the luminosity measurement of $6.8 \%$, we have a total 


\begin{tabular}{|l|c|}
\hline Uncertainty Source & Uncertainty (\%) \\
\hline \hline Trigger efficiency & 2 \\
Geometrical and kinematical acceptance & 4 \\
Lepton detection efficiency & 5 \\
Monte Carlo gluon radiation & 6 \\
Detector simulation of isolation & 4 \\
Top quark fragmentation & 2 \\
$E_{\text {T }}$ cut & 1 \\
Monte Carlo statistics & 3 \\
Luminosity & 6.8 \\
\hline Total & 13 \\
\hline
\end{tabular}

Table 5.7: Summary of the uncertainties in the detection efficiency $(\Delta \varepsilon / \varepsilon)$ and the luminosity $(\Delta L / L)$.

uncertainty given by

$$
\frac{\Delta\left(L \cdot \varepsilon_{\text {|ital }}\right)}{L \cdot \varepsilon_{\text {|rtal }}} \simeq 13 \%
$$

A summary of the various uncertainties is presented in Table 5.7.

\subsection{Background Study}

The $t \bar{t}$ dilepton signature can be mimicked by dilepton final states of Drell-Yan events $(\gamma / Z \rightarrow e e, \mu \mu$ and $\gamma / Z \rightarrow \tau \tau \rightarrow e \mu, e e, \mu \mu)$, diboson events $(W W$ or $W Z \rightarrow e \mu, e e, \mu \mu)$, or heavy flavor events $(b \bar{b}$ or $c \bar{c} \rightarrow e \mu, e e, \mu \mu)$. Events from ordinary QCD jets and $W+j e t s$ processes, with one or more misidentified leptons can also mimic the $t \bar{t}$ signature and are referred to as "fake dilepton" backgrounds. In what follows the estimation of the number of the various types of the background events expected in the dilepton data sample is presented. The backgrounds under consideration are

- $Z \rightarrow \tau \tau$

- $Z \rightarrow b \bar{b}$

- $W W$

- $W Z$ 


\begin{tabular}{|c|c|c|c|c|c|c|c|}
\hline & $\begin{array}{c}\sigma \cdot \mathrm{BR} \\
\mathrm{pb}\end{array}$ & $\varepsilon_{\text {Gcom }} \cdot P_{\mathrm{T}}$ & $\begin{array}{c}\varepsilon_{\text {lsol }} \\
\%\end{array}$ & $\begin{array}{c}\varepsilon_{I D} \\
\%\end{array}$ & $\begin{array}{c}\varepsilon_{\text {Event }} \\
\%\end{array}$ & $\begin{array}{c}\varepsilon_{\text {Trigger }} \\
\%\end{array}$ & $\varepsilon_{\text {Total }}$ \\
\hline$Z \rightarrow \tau \tau$ & 24.6 & $4.1 \times 10^{-2}$ & 98 & 85 & 7.4 & 98 & $(2.5 \pm 0.5) \times 10^{-3}$ \\
\hline$Z \rightarrow b \bar{b}$ & 44.1 & $6.6 \times 10^{-3}$ & 6.2 & 86 & 18 & 96 & $(6.1 \pm 1.4) \times 10^{-5}$ \\
\hline$W W$ & $2.82 \times 10^{-1}$ & $3.1 \times 10^{-1}$ & 92 & 85 & 71 & 95 & $(1.6 \pm 0.2) \times 10^{-1}$ \\
\hline$W Z$ & $9.33 \times 10^{-2}$ & $4.2 \times 10^{-1}$ & 90 & 84 & 25 & 95 & $(7.5 \pm 1.4) \times 10^{-2}$ \\
\hline$Z, \gamma \rightarrow e e, \mu \mu$ & 1095 & $1.0 \times 10^{-1}$ & 98 & 84 & 0.27 & 91 & $(2.0 \pm 1.6) \times 10^{-4}$ \\
\hline$b \bar{b}$ & $1.2 \times 10^{4}$ & $1.43 \times 10^{-3}$ & 8.9 & 87 & 5.6 & 99 & $(6.1 \pm 4.1) \times 10^{-5}$ \\
\hline
\end{tabular}

Table 5.8: The efficiencies for various background processes. The cross section multiplied by the braching ratio for each channel $\sigma \cdot \mathrm{BR}$ shown in the table is taken from the ISAJET calculation. The $\varepsilon_{\text {Total }}$ is relative to the number of events produced times the branching ratio for the channel to be considered. The statistical errors are shown for the total detection efficiencies $\varepsilon_{\text {Total }}$.

- $b \bar{b}$

- $Z, \gamma \rightarrow e e, \mu \mu$

- fakes.

The detection efficiency for the Drell-Yan, diboson and heavy flavor backgrounds, is divided into various terms, in the same way as was done for the $t \bar{t}$ signal in $\S 5.1$. As before, the ISAJET Monte Carlo event generator was used together with the detector simulation to determine the geometrical and kinematical acceptance, the efficiency of the lepton isolation cut, and the efficiency of the event topology (background rejection) cut. Also as before, we used lepton identification efficiencies measured for isolated leptons from $Z$ events, and trigger efficiencies measured for data collected with independent triggers. Table 5.8 shows the detection efficiencies for the real dilepton backgrounds. The overall efficiency of the event topology cut is shown in Table 5.9, along with its breakdown into separate efficiencies of the invariant mass window cut, back-to-back cut in $\phi$, and missing $E_{T}$ cut. The resulting number of background events expected in the data sample, both before and after the application of the event topology cut, is shown in Table 5.10. The cross sections used to predict the background yields of Table 5.10 are taken from the ISAJET calculation which are shown in Table 5.8. 


\begin{tabular}{|c|c|c|c|c|c|}
\hline & \multirow{3}{*}{$\begin{array}{c}\varepsilon_{\text {N/ass }} \\
\% \\
-\end{array}$} & \multirow{3}{*}{$\begin{array}{c}\begin{array}{c}\varepsilon_{\Delta \phi} \\
\%\end{array} \\
13\end{array}$} & \multirow{3}{*}{$\begin{array}{c}\varepsilon_{\text {Lr }_{\mathrm{T}}} \\
\% \\
\end{array}$} & \multirow{3}{*}{$\begin{array}{c}\begin{array}{c}\varepsilon_{\text {Evcnt }} \\
\%\end{array} \\
13 \pm 3.0\end{array}$} \\
\hline & & & & & \\
\hline $\boldsymbol{e \mu}$ & $Z \rightarrow \tau \tau$ & & & & \\
\hline & $Z \rightarrow b \bar{b}$ & - & 34 & - & $34 \pm 2.3$ \\
\hline & $W W$ & - & 79 & - & $79 \pm 5.4$ \\
\hline & $W Z$ & - & 75 & - & $75 \pm 8.6$ \\
\hline & $b \bar{b}$ & - & 20 & - & $20 \pm 13$ \\
\hline \multirow[t]{6}{*}{$e e, \mu \mu$} & $Z \rightarrow \tau \tau$ & 97 & 10 & 8.5 & $3.1 \pm 1.2$ \\
\hline & $Z \rightarrow b \bar{b}$ & 100 & 49 & 3.4 & $2.5 \pm 0.6$ \\
\hline & $W W$ & 83 & 77 & 77 & $61 \pm 6.7$ \\
\hline & $W Z$ & 12 & 92 & 62 & $8.2 \pm 3.0$ \\
\hline & $Z, \gamma \rightarrow e e, \mu \mu$ & 14 & 16 & 2.1 & $0.27 \pm 0.11$ \\
\hline & & 100 & 20 & 1.2 & $<0.2$ \\
\hline
\end{tabular}

Table 5.9: The efficiency of the event topology cut and its breakdown, for each background process. The errors are statistical. Note that only the back-to-back topology cut is applied in the $e \mu$ channel.

\begin{tabular}{|l||ccc|}
\cline { 3 - 4 } \multicolumn{2}{c|}{} & \multicolumn{2}{c|}{ Number of events at 4.1 $\mathrm{pb}^{-1}$} \\
\cline { 3 - 4 } \multicolumn{2}{c|}{} & before event cut & after event cut \\
\hline \multirow{4}{*}{} & $Z \rightarrow \tau \tau$ & 1.4 & $0.19 \pm 0.04$ \\
& $Z \rightarrow b \bar{b}$ & 0.030 & $0.010 \pm 0.002$ \\
& $W W$ & 0.15 & $0.12 \pm 0.01$ \\
& $W Z$ & 0.029 & $0.022 \pm 0.004$ \\
& $b \bar{b}$ & 1.5 & $0.3 \pm 0.2$ \\
& Fake & 1.6 & $0.6 \pm 0.4$ \\
\hline Total & & 4.7 & $1.2 \pm 0.5$ \\
\hline Data & & 4 & 1 \\
\hline \hline$e e, \mu \mu$ & $Z \rightarrow \tau \tau$ & 1.8 & $0.057 \pm 0.011$ \\
& $Z \rightarrow b \bar{b}$ & 0.03 & $0.0008 \pm 0.0001$ \\
& $W W$ & 0.12 & $0.071 \pm 0.007$ \\
& $W Z$ & 0.083 & $0.0066 \pm 0.0012$ \\
& $Z, \gamma \rightarrow e e, \mu \mu$ & 342 & $0.92 \pm 0.72$ \\
& $b \bar{b}$ & 3.9 & $<0.006$ \\
& Fake & 3.7 & $0.4 \pm 0.1$ \\
\hline Total & & 352 & $1.5 \pm 0.8$ \\
\hline Data & & 383 & 0 \\
\hline
\end{tabular}

Table 5.10: The expected number of background events at $4.1 \mathrm{pb}^{-1}$ before and after the event topology cut with the statistical error. 


\section{The event topology cut efficiency, $\varepsilon_{\text {Event }}$, for Drell-Yan events}

We note that in order for Drell-Yan events to pass the event topology cut, they must have an intermediate $\gamma$ or $Z$ produced at high $P_{\mathrm{T}}$. Such events will have dileptons which are not back-to-back, and will also have jets. The jets can give rise to significant missing transverse energy when a large fluctuation in the calorimeter measurement occurs. In this case, the Drell-Yan events are likely to pass both the back-to-back cut and the $\mathbb{E}_{\mathrm{T}}$ cut. More than $85 \%$ of the Monte Carlo ee and $\mu \mu$ Drell-Yan events passing the event topology cut have $P_{\mathrm{\gamma}}^{Z, \gamma}>50 \mathrm{GeV} / c$. Only a small fraction $(2 \%)$ of Drell-Yan events are produced at such high $P_{\top}$. Since the tail of the $P_{\mathrm{T}}$ distribution is important in this case, it is necessary to generate more high $P_{\mathrm{T}}^{Z, \gamma}$ Drell-Yan events to determine the $\varepsilon_{\text {Event }}$ more accurately. It is also desirable to use the $P_{\text {I }}$ distribution independent of the Monte Carlo prediction. Therefore the $\varepsilon_{\text {Evem }}$ was determined as a function of $P_{\mathrm{T}}^{Z, \gamma}$ by generating only Drell-Yan events which have high $P_{\mathrm{T}}^{Z, \gamma}$ in the interested region, and by convoluting the efficiency curve with the shape of the $P_{\mathrm{T}}^{Z}$ distribution measured for $Z$ events. Recall that $Z$ events dominate the Drell-Yan background because of the high $E_{\mathrm{T}}$ cut on the leptons. Figure 5.19 shows $\varepsilon_{\text {Wirm }}$ as a function of $P_{\mathrm{T}}^{Z, \gamma}$. Figure 5.20 shows the $P_{\mathrm{T}}^{Z}$ distribution from CDF $Z$ events with the Monte Carlo distribution showing a discrepancy from the CDF data.

\section{$b \bar{b}$ background}

A problem in the estimation of the $b \bar{b}$ background is discussed here. The problem is though not significant in the current case because the remaining $b \bar{b}$ background is kept very small anyway.

The $b \bar{b}$ can be produced by two processes:

- direct pair production $(p \bar{p} \rightarrow b \bar{b})$

- gluon splitting process $(g \rightarrow b \bar{b})$. 
A total of $3.1 \mathrm{pb}^{-1} b \bar{b}$ Monte Carlo events were generated using ISALEP [67], a special version of ISAJET.

Each process shows its specific feature in the azimuthal opening angle between an electron and a muon, $\Delta \phi_{c \mu}$. Figure 5.21 (a) compares $\Delta \phi_{e \mu}$ observed in the low $E_{\mathrm{T}}\left(P_{\mathrm{T}}\right)$ CDF central $e \mu$ events with the Monte Carlo data. The CDF had the central $e \mu$ trigger with threshold at $5 \mathrm{GeV} /(c)$ on both leptons for this type of events. For the plot, $E_{\mathrm{T}}\left(P_{\mathrm{T}}\right)$ thresholds were lowered from $15 \mathrm{GeV} /(c)$ down to $7 \mathrm{GeV} /(c)$ on both leptons and no explicit cone isolation was applied to retain $b \bar{b}$ events. The contribution from each $b \bar{b}$ production process is separately shown in Figure 5.21 (b) and (c). The $\Delta \phi_{e, \mu}$ distribution from the gluon splitting process is characterized by the fact that more events cluster in $30^{\circ}-100^{\circ}$ region compared with the direct production process because the gluon splitting process does not necessarilly produce a $b \bar{b}$ pair with a back-to-back configuration contrary to the direct pair production process. Since the cut $\Delta \phi_{c, \mu}<160^{\circ}$ is used as an event topology cut for $e \mu$ events, the background estimation is sensitive not only to the total $b \bar{b}$ rate but also to the relative rate of the two processes.

We understand that there is a large uncertainty in the theoretical calculations of $\sigma(p \bar{p} \rightarrow b \bar{b})(\sim 50 \%)[22]$. Furthermore it is difficult to calculate reliably the rate of the gluon splitting, which is basically the higher order process. Actually we observe a disagreement between data and Monte Carlo. We observed 140 low $E_{\uparrow}\left(P_{\uparrow}\right) e \mu$ events in the CDF data and the Monte Carlo prediction is $\sim 290$ events at $4.1 \mathrm{pb}^{-1}$ (100 events from the direct production and 190 from the gluon splitting). The discrepancy is larger than $100 \%$.

Furthermore, the Monte Carlo $\Delta \phi_{r, \mu}$ distribution does not reproduce the data as shown in Figure 5.21 (a). Although we have to subtract the fake background from the $e \mu$ events in the data to obtain the real shape of $\Delta \phi_{e, \mu}$ distribution, this disagreement both in the rate and the shape indicates that it is necessary to measure the $b$ quark production cross section in dilepton events for the proper normalization. Since CDF has measured the $b$ quark production cross section using the inclusive electron rate and 
the exclusive decays, we can normalize the total number of b's in Monte Carlo to the measured value. However we do not know the relative contribution from the direct pair production and the gluon splitting process, which is important for dilepton events. Thus we need to know the $b$ quark production in such a way as is sensitive to the angle correlation between two leptons, which has not yet been performed. The number of events shown in Table 5.10 for the $b \bar{b}$ background is simply a face value obtained from the ISAJET Monte Carlo ignoring a possible incorrect normalization.

\section{Fake dilepton}

The "fake dilepton" backgrounds were calculated separately for each of the 8 dilepton categories using a common method. For concreteness, we consider the $e \mu$ case in the following. Let $N$ be the number of $e \mu$ pairs in the final sample. First, we estimate the number of $e \mu$ pairs with a fake muon. The high $P_{\Gamma}$ fake muons which are hadrons usually rejected by the calorimeter minimum ionizing cut are additionally introduced to the sample by removing the minimum ionizing cut, resulting in an increased number of $e \mu$ pairs $N^{\prime}$. Since muons in each sample of $N$ and $N^{\prime}$ pairs consists of real muons and fake muons, we can write

$$
\begin{aligned}
N & =N_{\text {real }}+N_{\text {fake: }}, \\
N^{\prime} & =N_{\text {real }}^{\prime}+N_{\text {fake: }}^{\prime},
\end{aligned}
$$

where $N_{\text {rcal }}$ is the number of real muons in the sample of $N e \mu$ pairs, $N_{\text {fake }}$ is the number of fake muons in the same sample, $N_{\text {real }}^{\prime}$ is the number of real muons in the sample with the relaxed cut on muons and $N_{\text {fakc }}^{\prime}$ is the number of fake muons in the same sample. If we know the probability $p_{\text {real }}$ that a real muon passes the calorimeter minimum ionizing cut, and the same probability for a fake muon, $p_{\text {fake: }}$, we can estimate $N_{\text {fake }}=p_{\text {fakc }} N_{\text {fake }}^{\prime}$ by solving the following equations,

$$
N=p_{\text {real }} N_{\text {rcal }}^{\prime}+p_{\text {fake }} N_{\text {fakc: }}^{\prime}
$$




$$
N^{\prime}=N_{\text {real }}^{\prime}+N_{\text {fake }}^{\prime}
$$

The "fake probability", $p_{\text {fake }}$, per isolated track is obtained from a background sample of events collected with a jet trigger with an $E_{r}$ threshold of $20 \mathrm{GeV}$ in which those events with large missing $E_{\mathrm{T}}$, and dimuons are removed to avoid a contamination of real muons from $W$ and $Z$ events. It is possible that the muon candidates which pass all the selection cuts in this jet sample are real muons, so the number obtained should be considered as an upper limit of the true $p_{\text {rake: }}$ The $p_{\text {rcal }}$ is known from $Z$ events. Thus we can estimate a fake muon fraction of the final sample,

$$
f_{\text {fake }}^{\prime \prime}=\frac{N_{\text {fake }}}{N}
$$

This accounts for real $e$ plus fake $\mu$ and fake $e$ plus fake $\mu$.

The same procedure is repeated for the electron side. Here all the electron selection cuts are removed except $E_{\Gamma}$ and isolation cuts. The $p_{\text {rakc }}$ and $p_{\text {rcal }}$ are estimated using a jet sample and $Z$ sample. Following the same steps, we derive the fake electron fraction of the final sample $f_{\text {fake: }}^{\text {f }}$.

Finally the number of total fake $e \mu$ pairs is calculated by

$$
N_{\text {fakc: }}^{c \mu}=N\left(f_{\text {fake: }}^{\prime \prime}+f_{\text {fakt: }}^{c}-f_{\text {fake: }}^{\mu} f_{\text {fake: }}^{c}\right)
$$

The last term corrects for a double counting of fake $\mu+$ fake $e$ pairs. Table 5.11 shows the values of $p_{\text {rcal }}$ and $p_{\text {rake }}$ for the various lepton categories. The results for the number of fake events expected are shown in Table 5.12.

The $p_{\text {rake }}$ is estimated for isolated fake leptons to remove its possible physics source dependence. Because the lepton identification cut efficiency is dependent on the isolation as explained in $§ 5.1 .2$ and because the isolation of a particle is usually different for the physics process considered, the $p_{\text {rake: }}$ would change from sample to sample. In order to reduce this possible variation of $p_{\text {rak: }}$ according to the physics source, the isolation 


\begin{tabular}{|l|c|c|}
\hline Lepton Type & $p_{\text {real }}$ & $p_{\text {fake }}$ \\
\hline CE (tight) & $0.85 \pm 0.03$ & $0.004 \pm 0.002$ \\
CE (loose) & $0.96 \pm 0.01$ & $0.059 \pm 0.007$ \\
PE & $0.79 \pm 0.04$ & $0.09 \pm 0.01$ \\
\hline MU & $0.98 \pm 0.01$ & $0.11 \pm 0.04$ \\
MI & $0.98 \pm 0.01$ & $0.010 \pm 0.004$ \\
\hline
\end{tabular}

Table 5.11: The probabilities for real leptons $\left(p_{\text {rcal }}\right)$, and for fake leptons $\left(p_{\text {fake }}\right)$ to pass the identification cut. Isolated EM clusters in a jet event sample are considered as fake electrons. As fake muons, all the isolated tracks in a jet event sample are considered. The statistical errors are shown in this table.

\begin{tabular}{|l|c|c|}
\hline Dilepton Type & Before Topology Cuts & After Topology Cuts \\
\hline \hline CEMU+CEMI & $0.9 \pm 0.4$ & $0.3 \pm 0.2$ \\
PEMU+PEMI & $0.7 \pm 0.4$ & $0.3 \pm 0.3$ \\
\hline Total $e \mu$ & $1.6 \pm 0.6$ & $0.6 \pm 0.4$ \\
\hline \hline CECE & $1.2 \pm 0.2$ & $0.09 \pm 0.04$ \\
CEPE & $2.0 \pm 0.8$ & $0.10 \pm 0.05$ \\
MUMU+MUMI & $0.5 \pm 0.3$ & $0.10 \pm 0.05$ \\
\hline Total $e e+\mu \mu$ & $3.7 \pm 0.9$ & $0.4 \pm 0.1$ \\
\hline
\end{tabular}

Table 5.12: The number of fake dilepton backgrounds expected in $4.1 \mathrm{pb}^{-1}$. The errors are statistical. 
requirement was kept in the $p_{\text {faks }}$ estimation. 

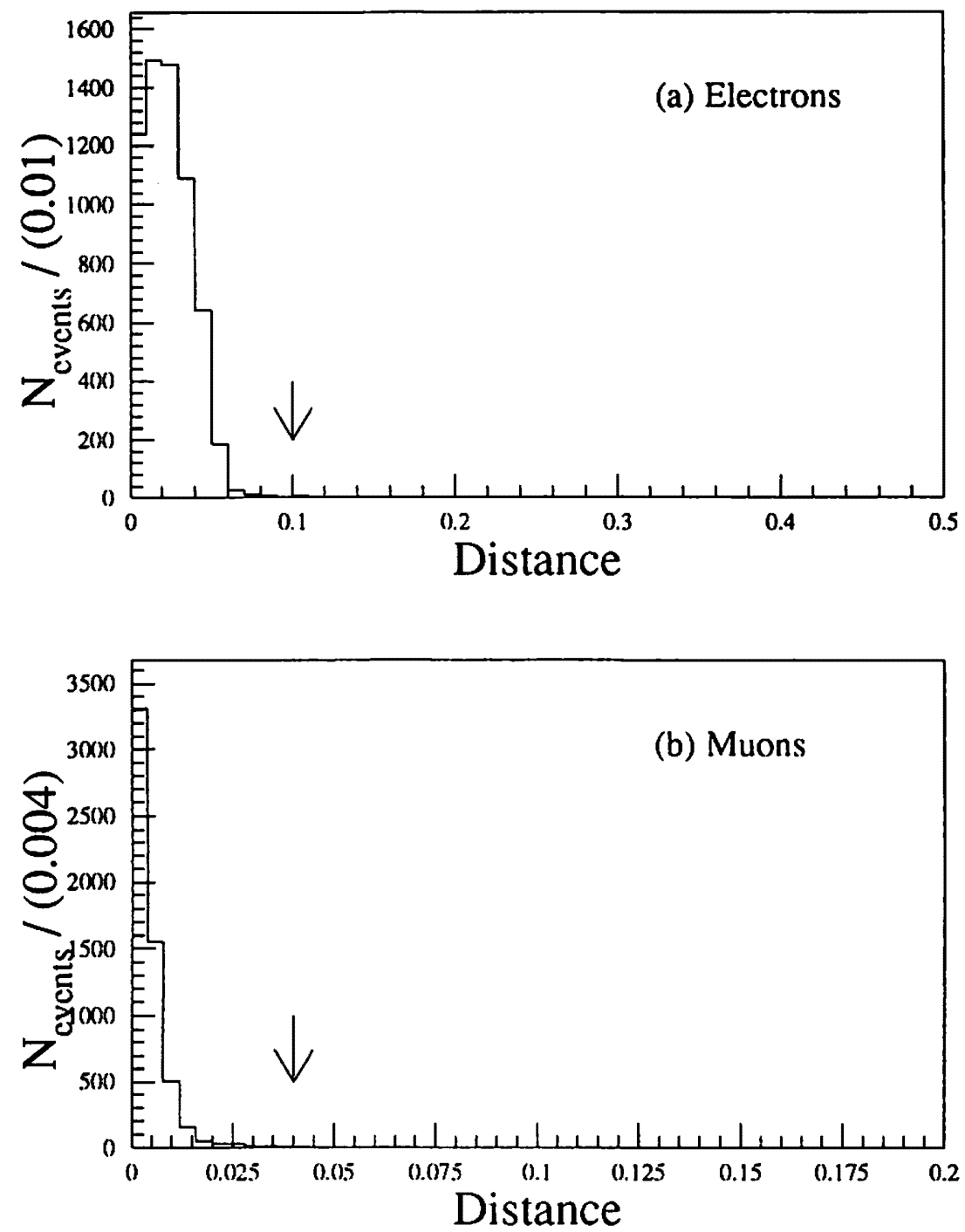

Figure 5.1: The distance in $\eta-\phi$ space between GENP leptons from top quark decays and the nearest reconstructed lepton for (a) electrons with $E_{\mathrm{T}}>15 \mathrm{GeV}$ and (b) muons with $P_{\mathrm{T}}>15 \mathrm{GeV} / c . M_{\text {L,p }}=90 \mathrm{GeV} / c^{2}$. 

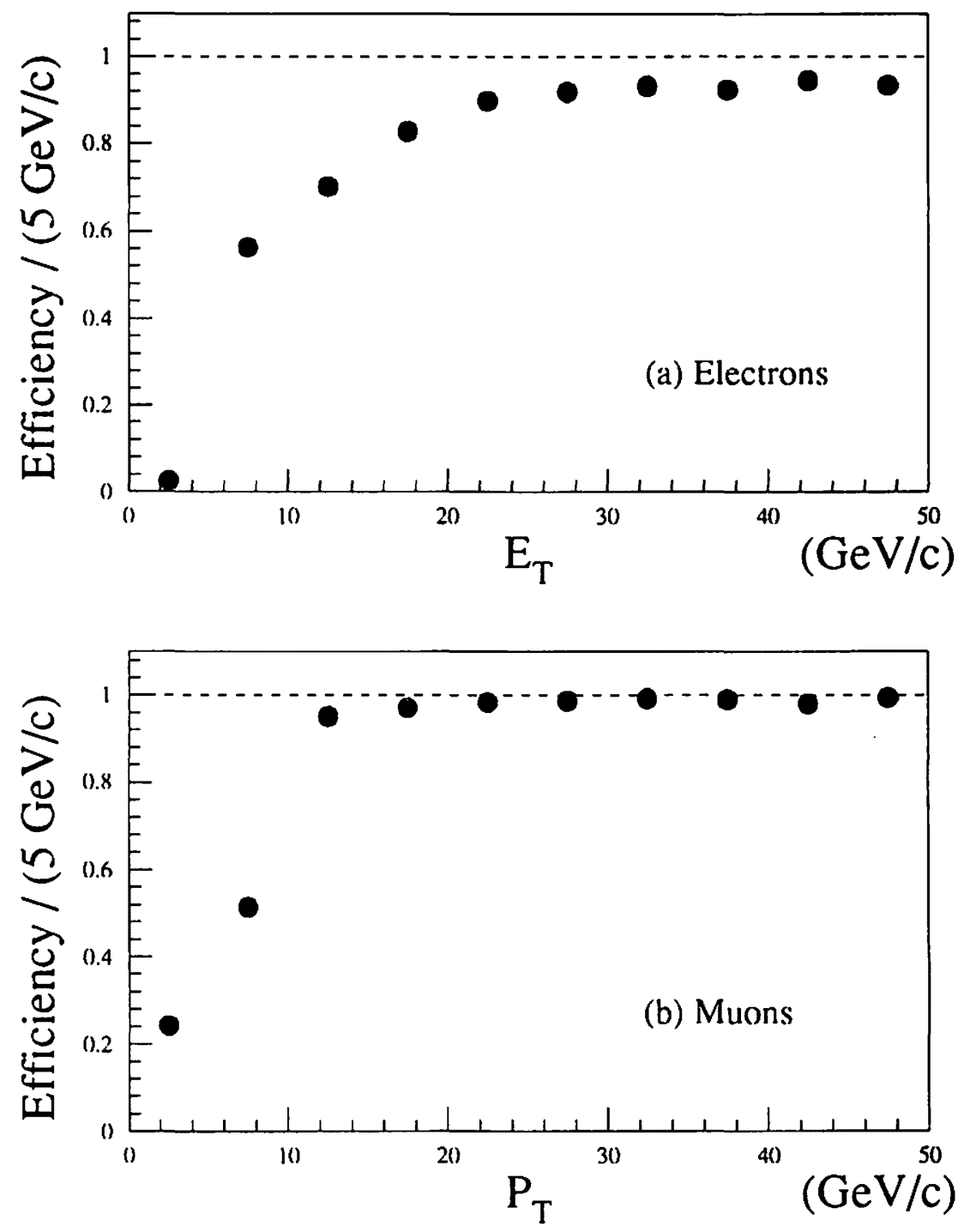

Figure 5.2: Efficiency for a top lepton to have a matched lepton reconstructed by the offline codes as a function of lepton $E_{\uparrow} / P_{\mathrm{T}}$ for (a) electrons and (b) muons. $M_{\mathrm{Lop}}=90$ $\mathrm{GeV} / c^{2}$. 


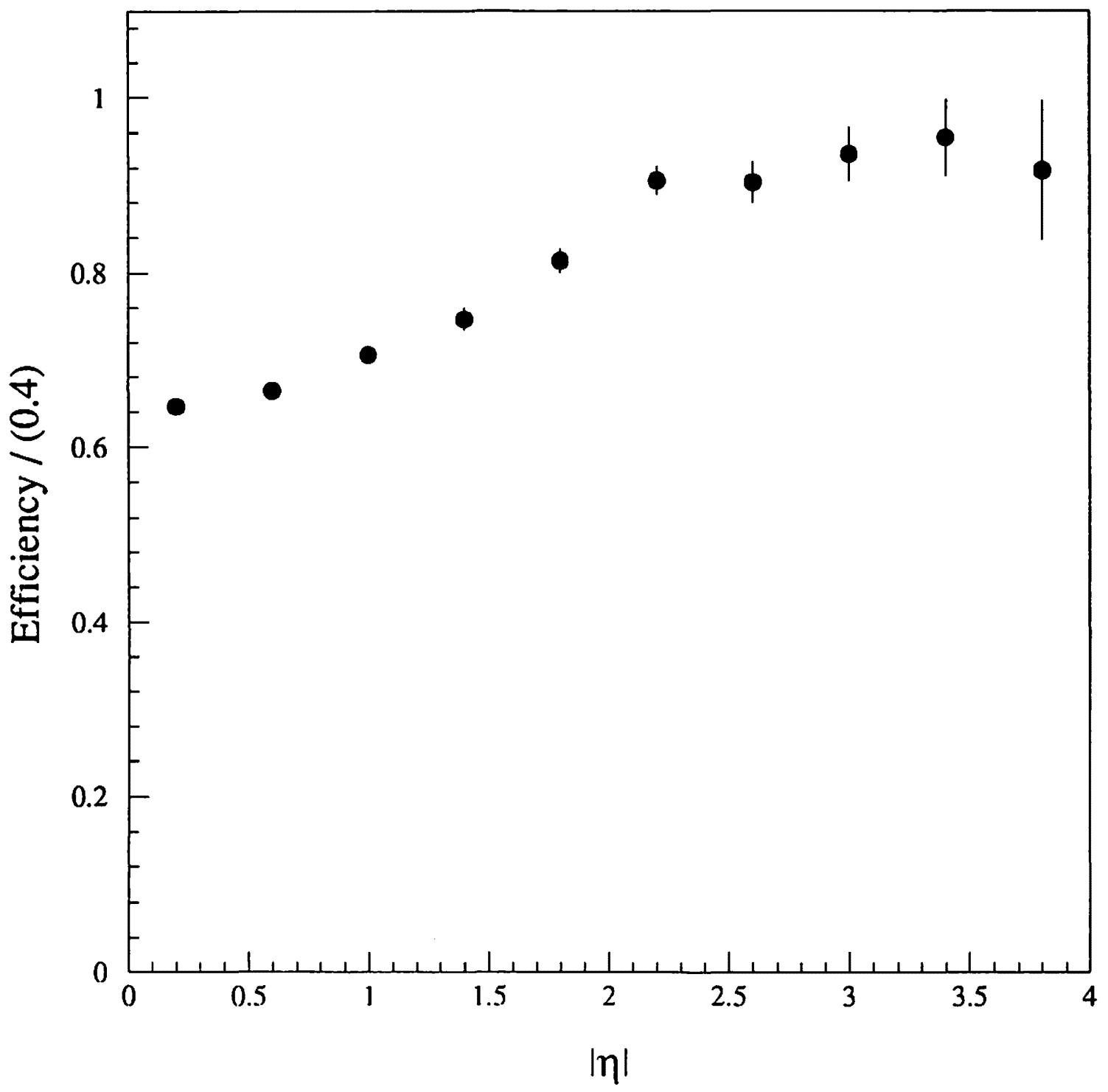

Figure 5.3: The isolation cut efficiency for electrons from $M_{\text {top }}=90 \mathrm{GeV} / c^{2}$ ISAJET Monte Carlo data as a function of $\eta$. The detector simulation is not applied. 


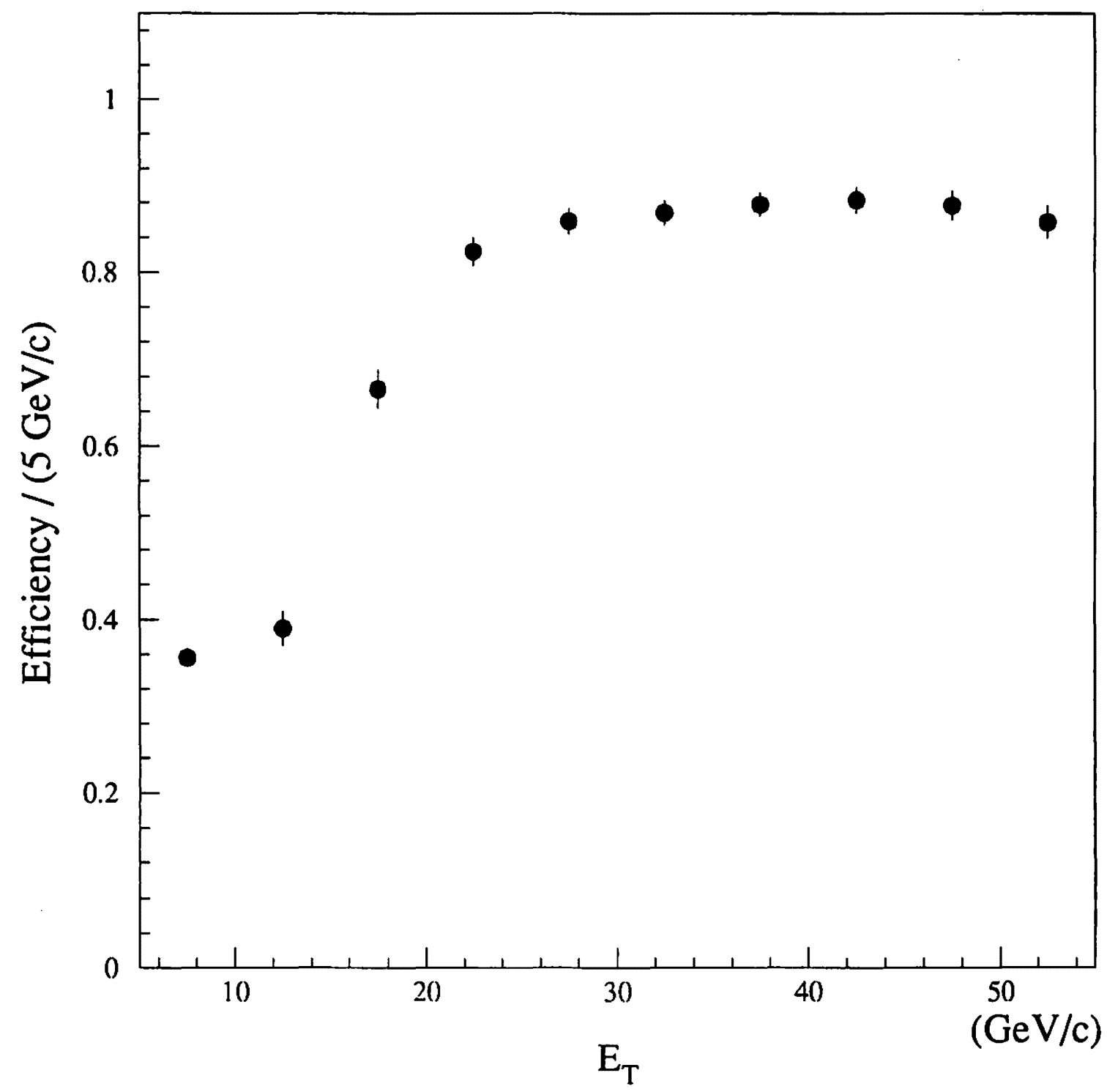

Figure 5.4: The isolation cut efficiency for the central electrons from $M_{\mathrm{top}}=90 \mathrm{GeV} / \mathrm{c}^{2}$ ISAJET Monte Carlo data as a function of $E_{\uparrow}$. The detector simulation is not applied. 

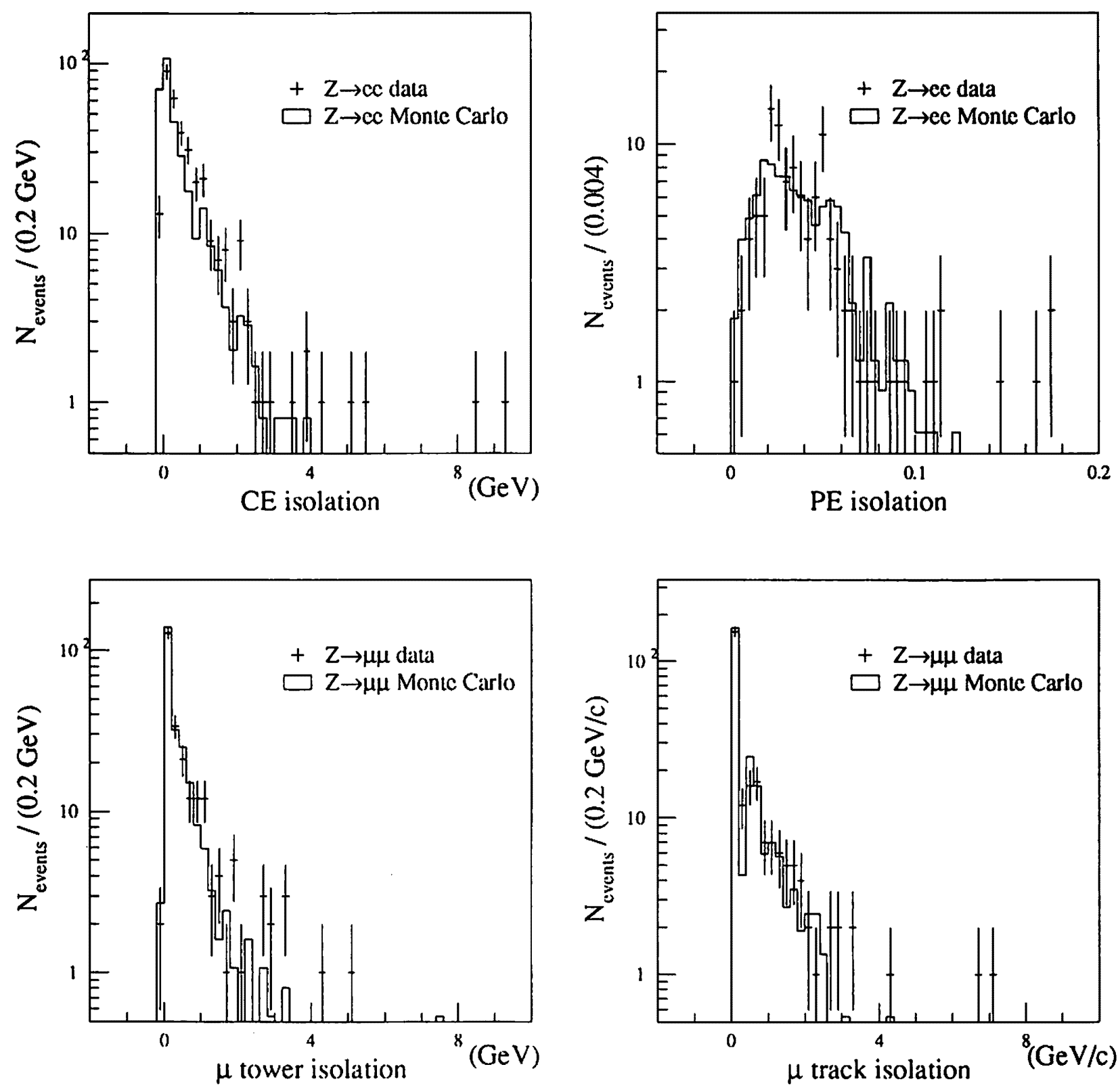

Figure 5.5: Comparison of the lepton isolation distributions for the CDF $Z$ data and the ISAJET + CDFSIM $Z$ Monte Carlo. For the Monte Carlo events, the cut $E_{\mathrm{T}}\left(P_{\mathrm{T}}\right)>15$ $\mathrm{GeV}(\mathrm{GeV} / \mathrm{c})$ is imposed on leptons. For leptons in the CDF data, all the lepton selection cuts except the isolation are required. 

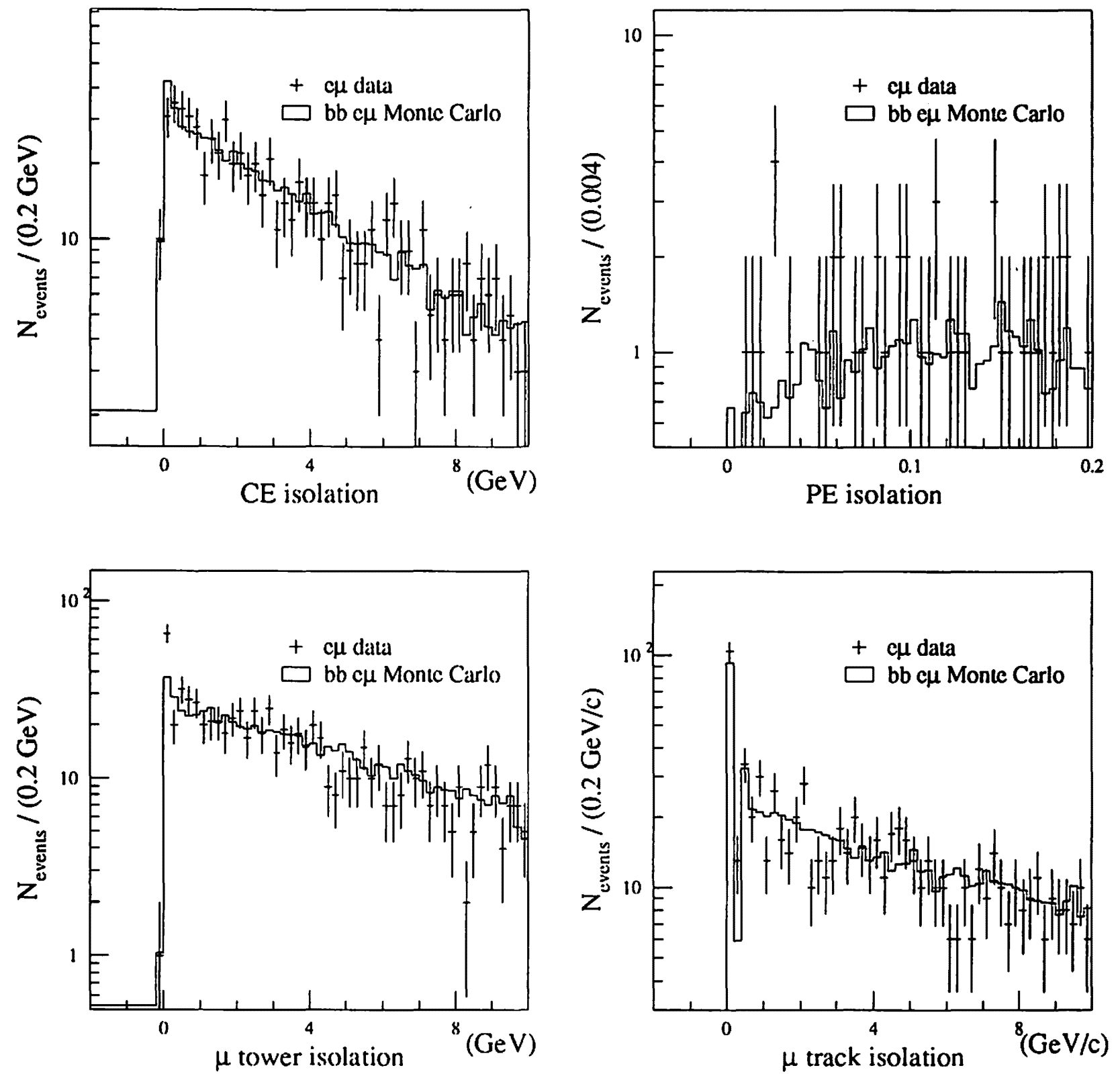

Figure 5.6: Comparison of the lepton isolation distributions for the low $E_{\mathrm{T}}$ CDF $e \mu$ events and the ISAJET + CDFSIM $b \bar{b}$ Monte Carlo. For the Monte Carlo events, the cut $E_{\mathrm{T}}\left(P_{\mathrm{r}}\right)>7 \mathrm{GeV}(\mathrm{GeV} / c)$ is imposed on leptons. For leptons in the CDF data, all the lepton selection cuts except the isolation requirement are required. 


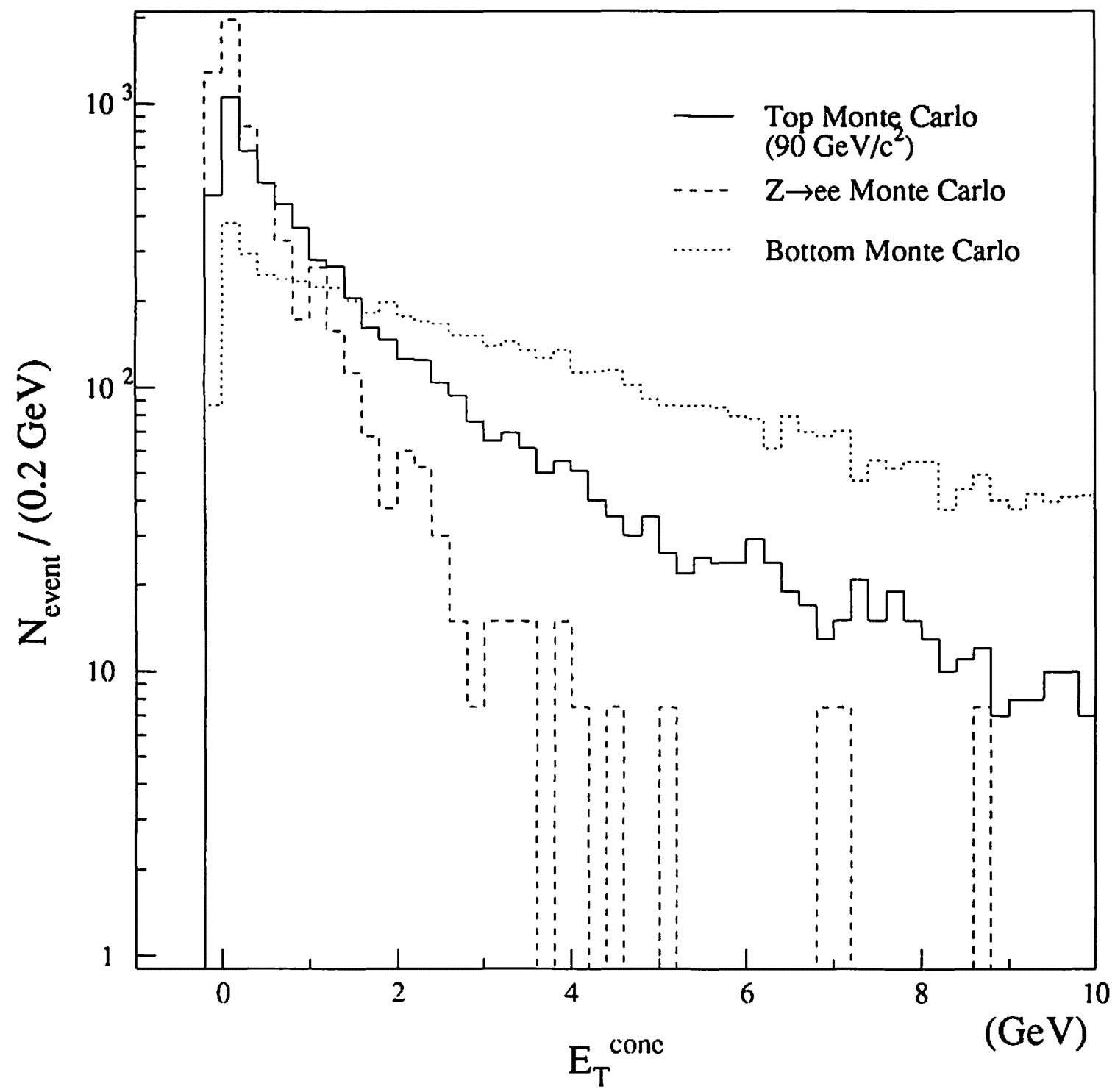

Figure 5.7: The isolation distributions for central electrons from the $t \bar{t}$ Monte Carlo with $M_{\text {top }}=90 \mathrm{GeV} / c^{2}$, the CDF $Z \rightarrow e e$ Monte Carlo and the $b \bar{b}$ Monte Carlo. 

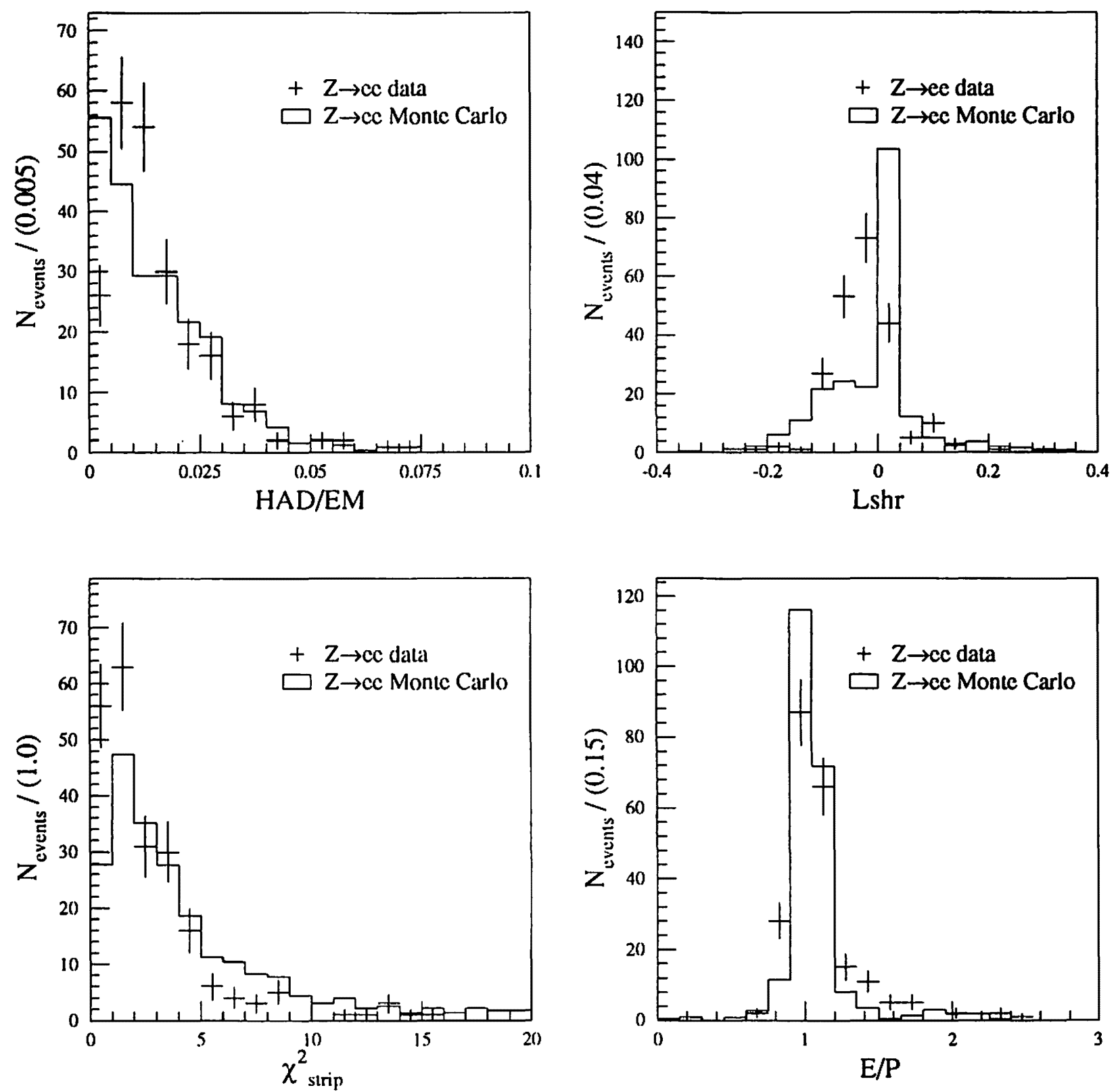

Figure 5.8: Comparison of the central electron identification variables for the CDF and Monte Carlo $Z \rightarrow$ ee events. 

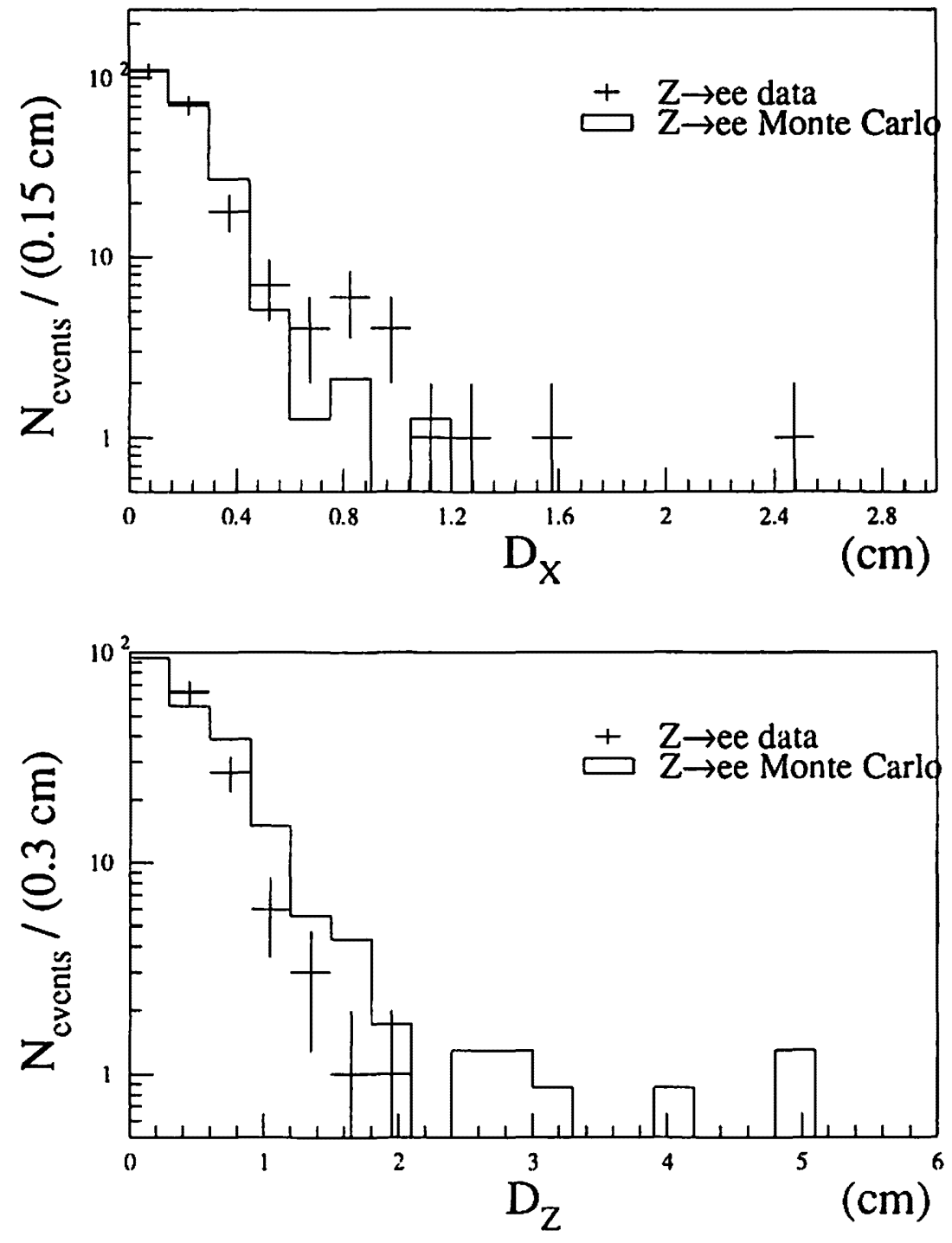

Figure 5.9: Comparison of the central electron identification variables for the CDF and Monte Carlo $Z \rightarrow e e$ events. 


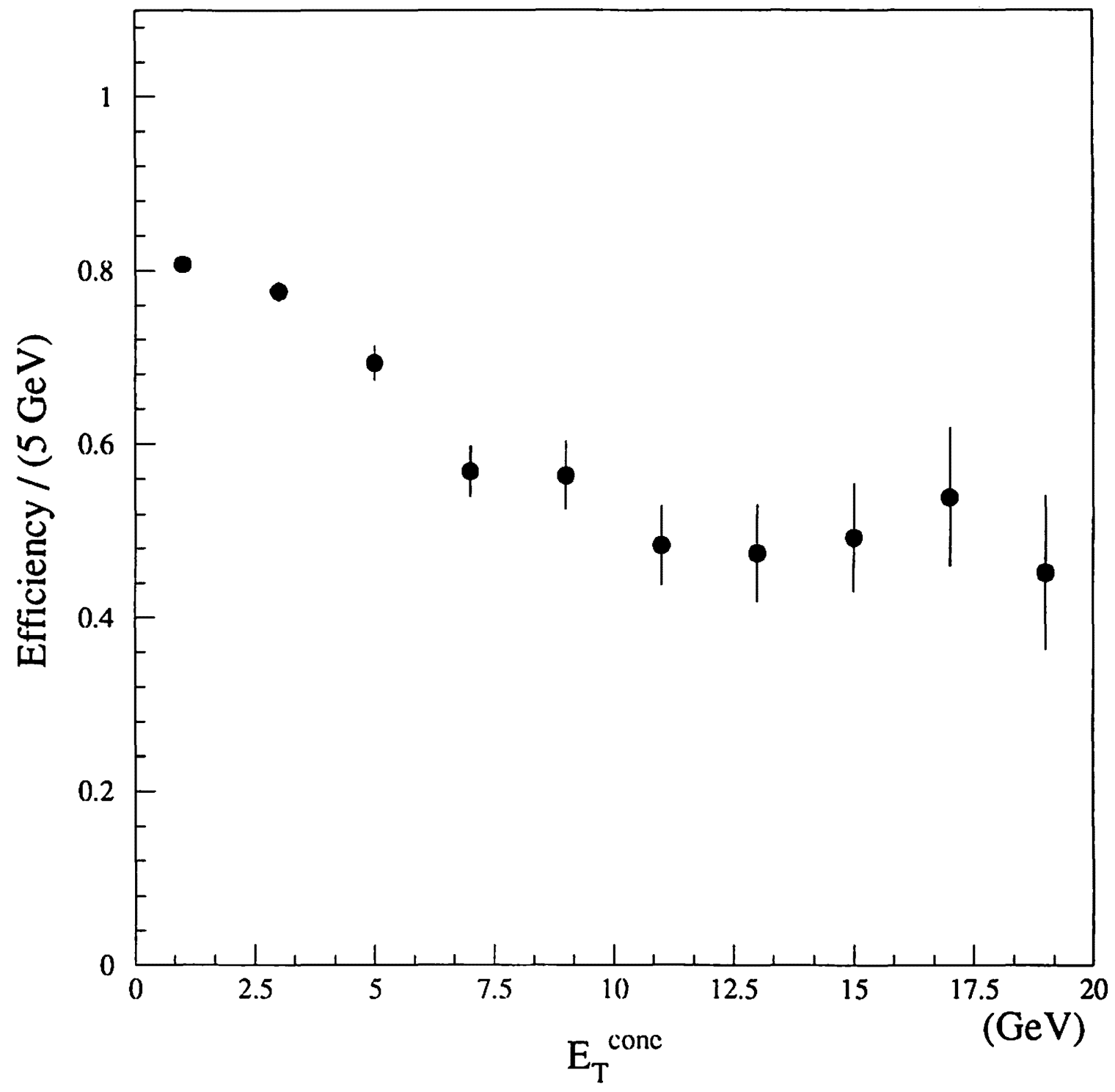

Figure 5.10: The central electron identification efficiency as a function of the isolation calculated from $M_{\text {top }}=90 \mathrm{GeV} / c^{2}$ ISAJET Monte Carlo + CDFSIM data. 


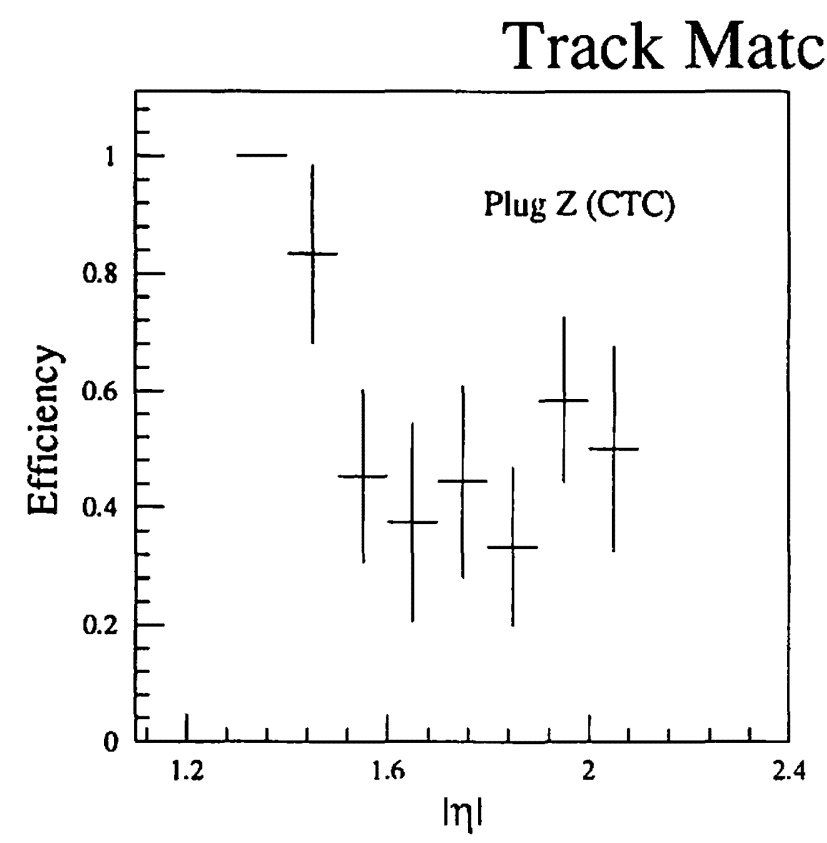

hing Efficiency
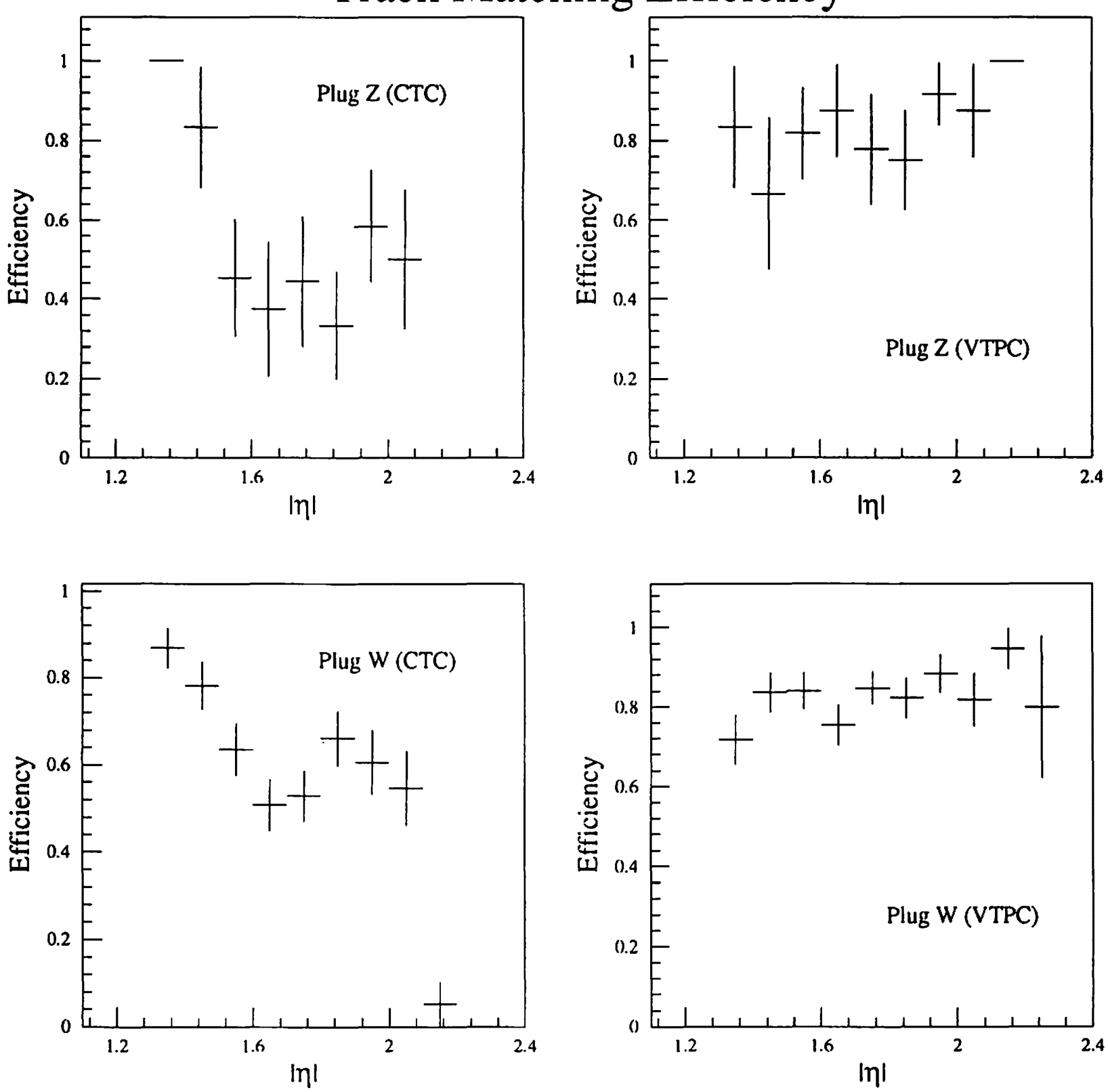

Figure 5.11: The CTC and VTPC track matching efficiency as a function of $\eta$ for the plug $Z$ and $W$ events. 


\section{Track Matching Efficiency}
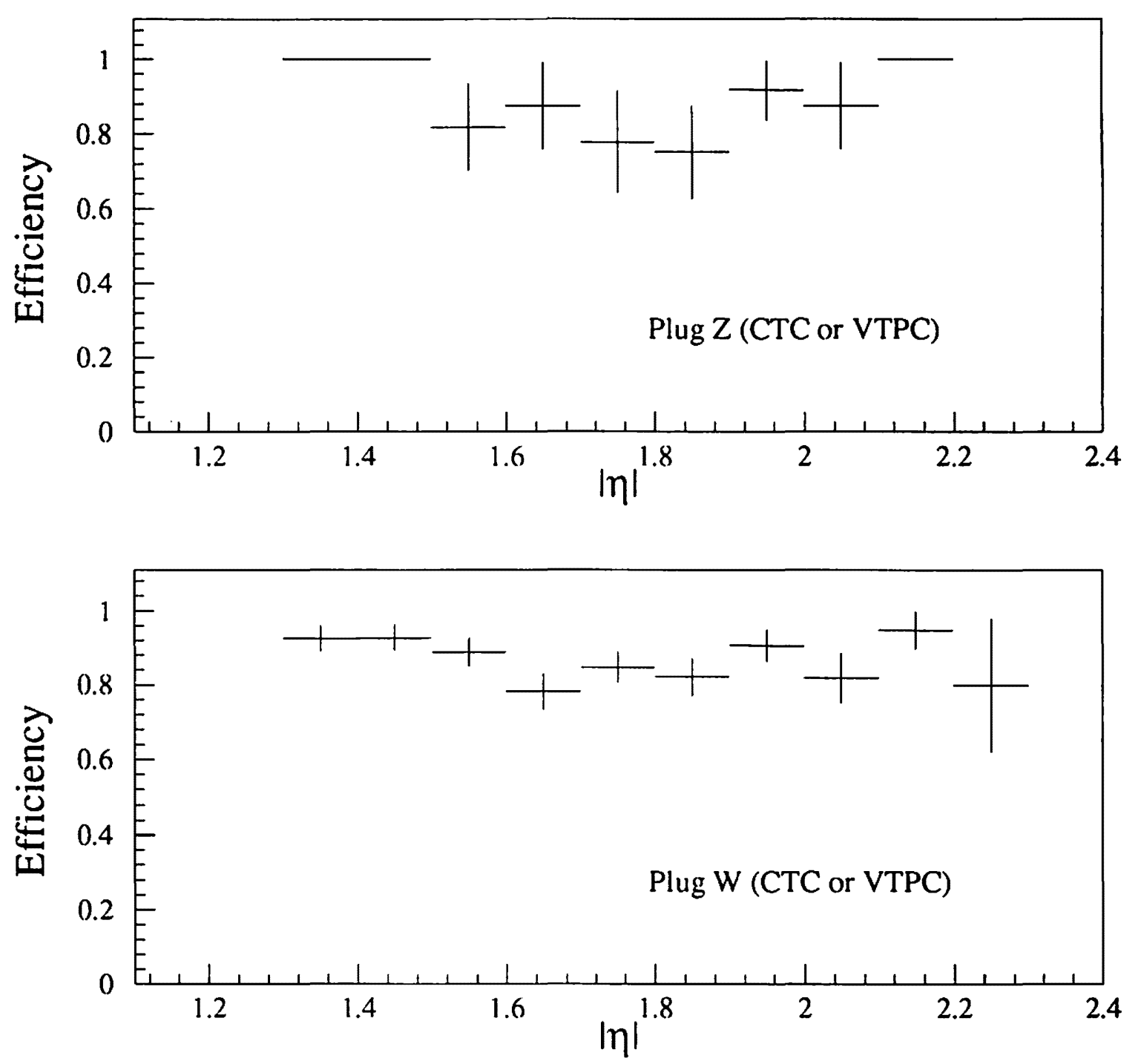

Figure 5.12: The track matching (CTC or VTPC) efficiency as a function of $\eta$ for the plug $Z$ and $W$ events. 


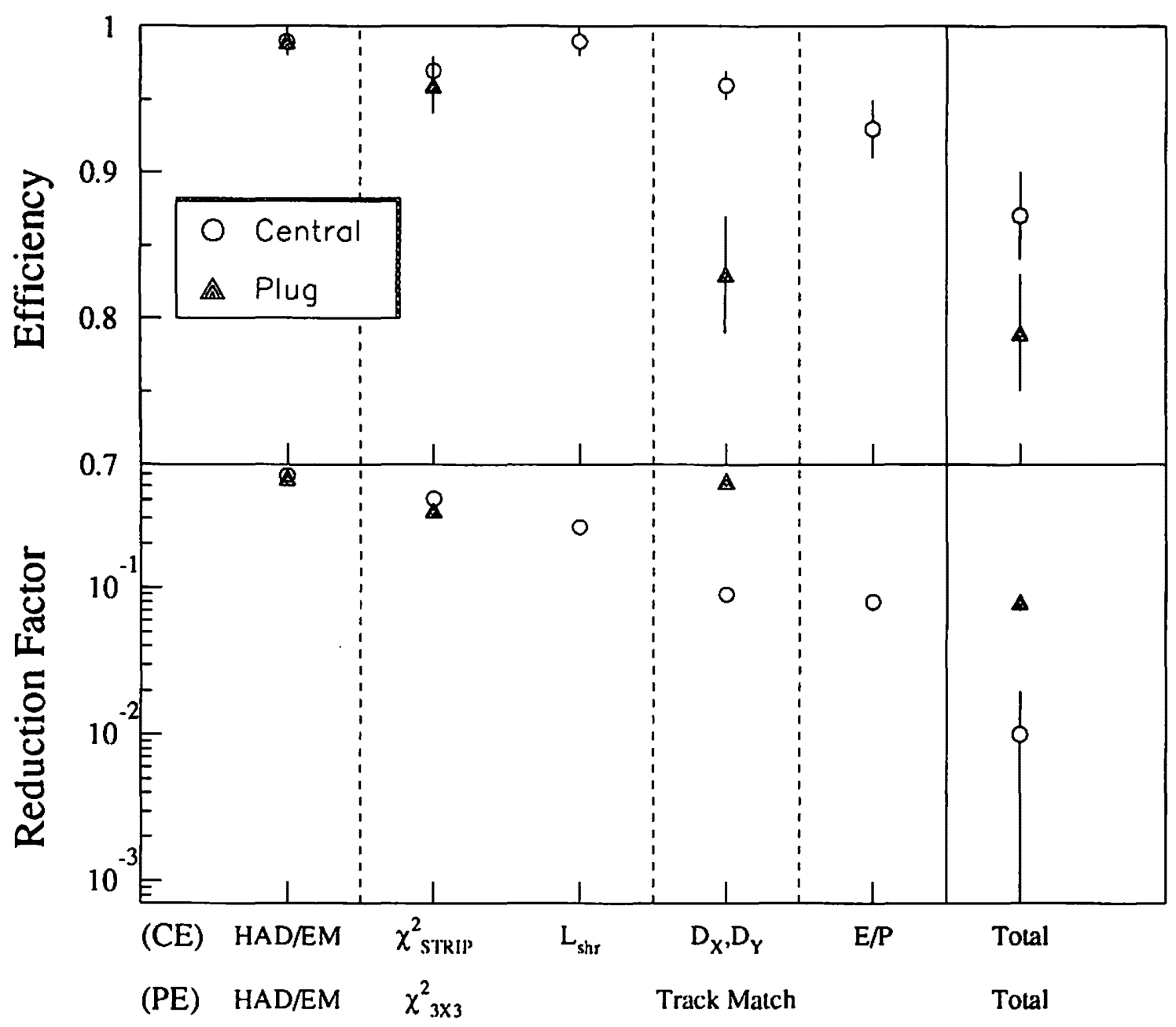

Figure 5.13: The electron identification cut efficiency and the background reduction factor of the cut for the central electron and the plug electron. 

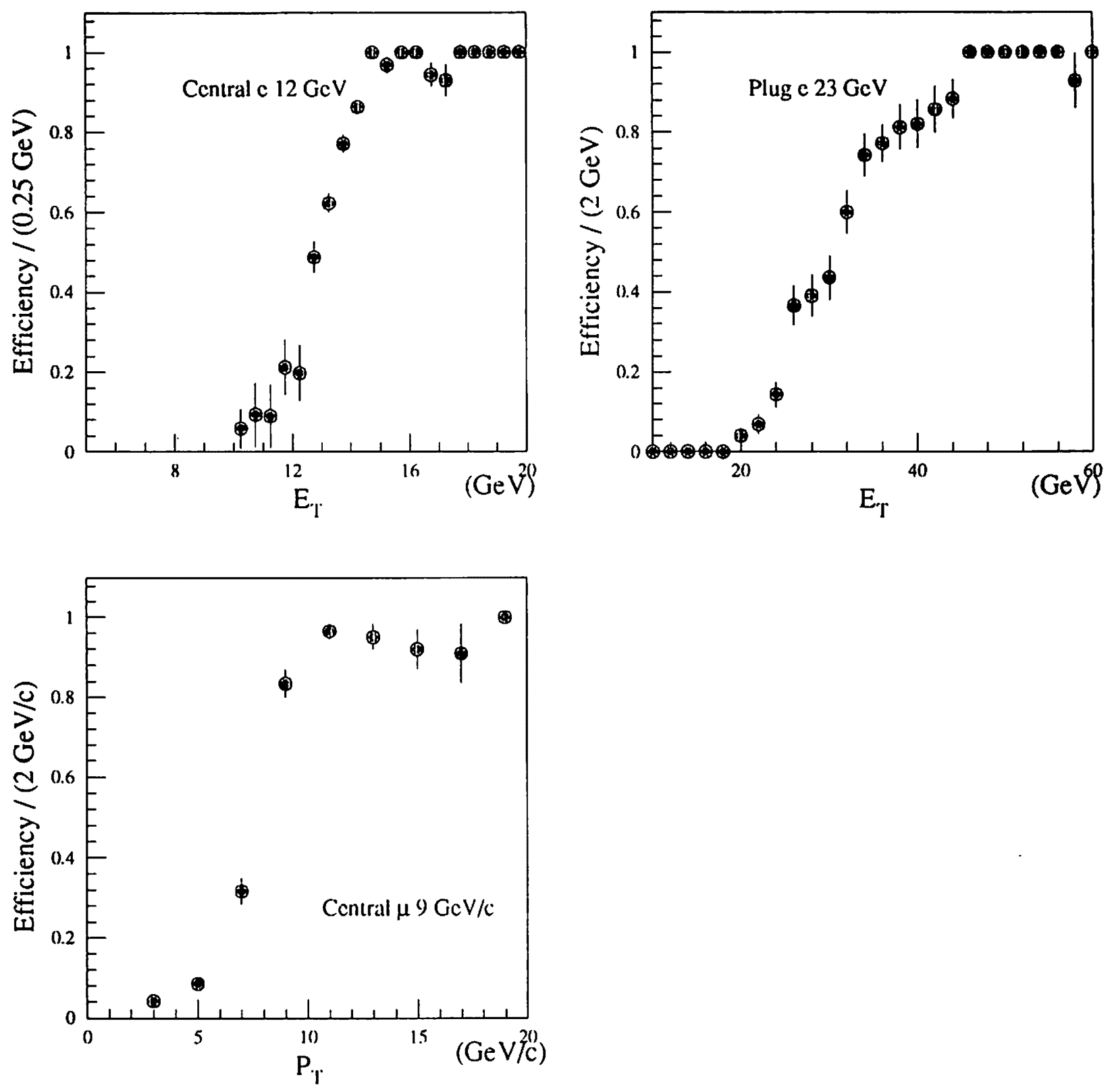

Figure 5.14: The trigger efficiencies as a function of electron $E_{\uparrow}$ or muon $P_{r}$ for the central electron, the plug electron and the central muon triggers. 


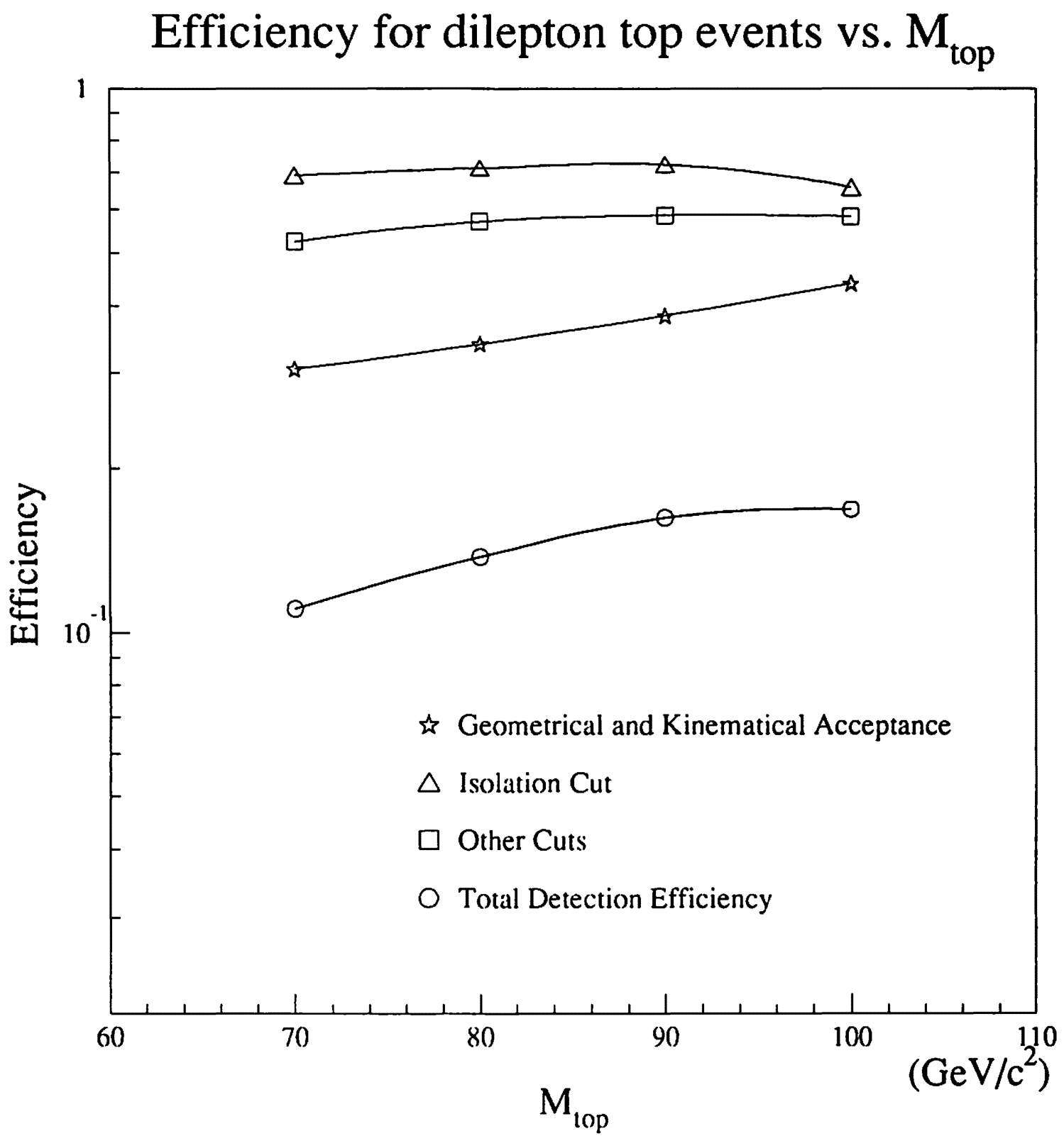

Figure 5.15: The top quark mass dependence of the cut efficiencies and the total detection efficiency. 

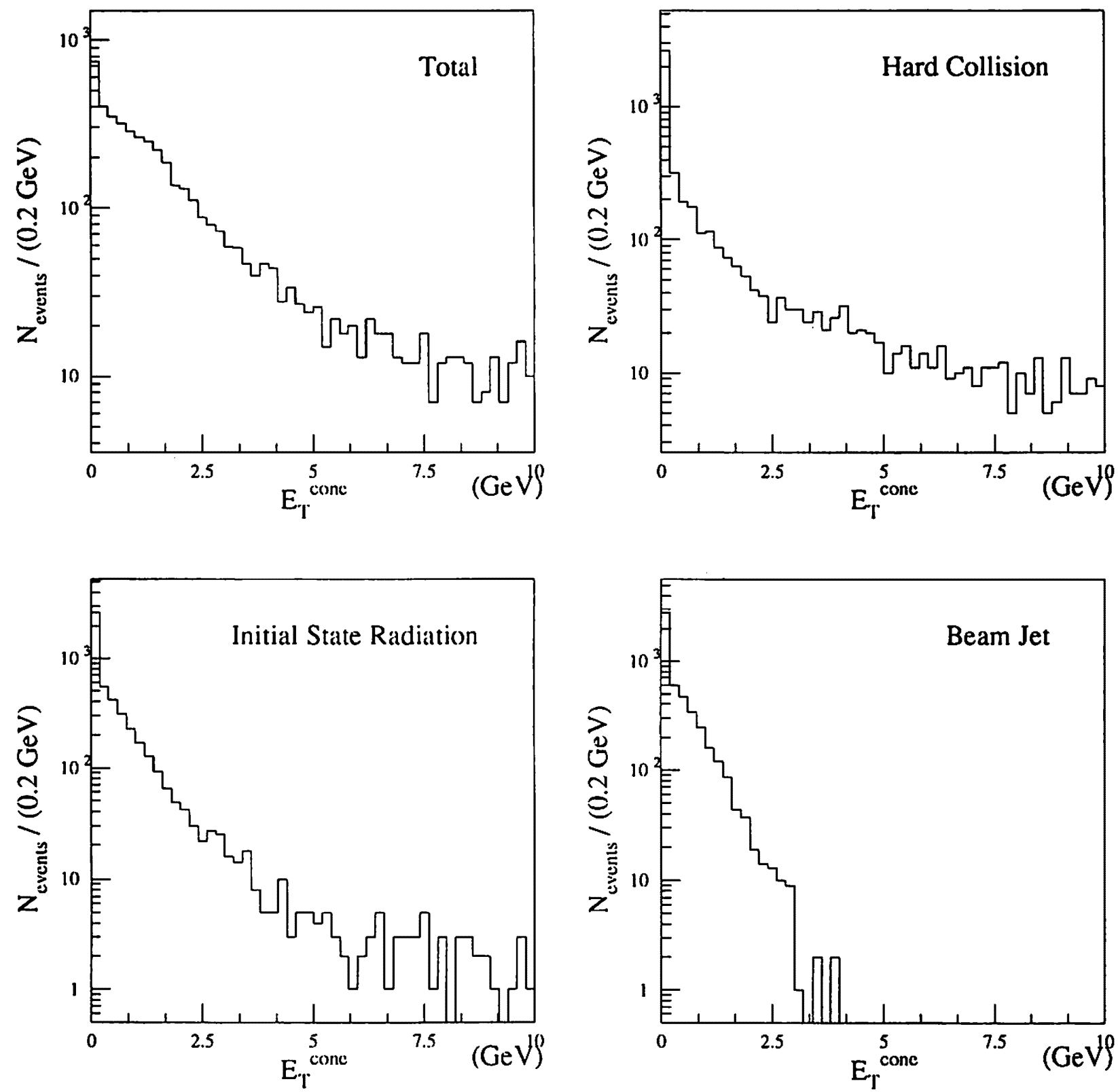

Figure 5.16: The GENP isolation for the central electrons (a), and contributions from (b) the hard collision, (c) initial state radiation, and (d) beam jet. The $t \bar{t}$ Monte Carlo with $M_{\text {(o) }}=90 \mathrm{GeV} / c^{2}$ is used for the plots. 

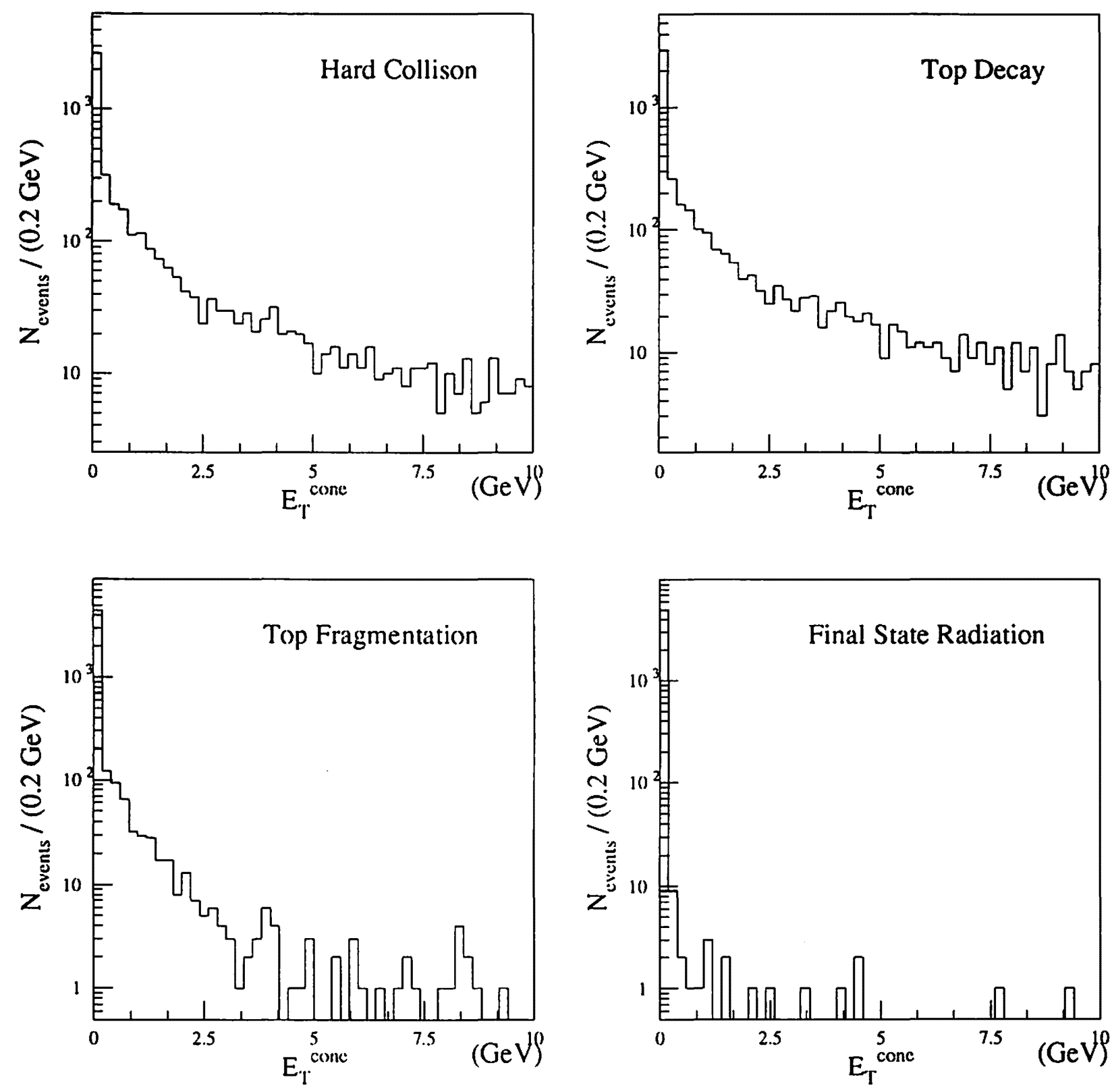

Figure 5.17: The GENP isolation from the hard collision for the central electron (a), and the contributions from (b) the top quark decay, (c) top quark fragmentation, and (d) final state radiation. The $t \bar{t}$ Monte Carlo with $M_{\text {cop }}=90 \mathrm{GeV} / c^{2}$ is used for the plots. 


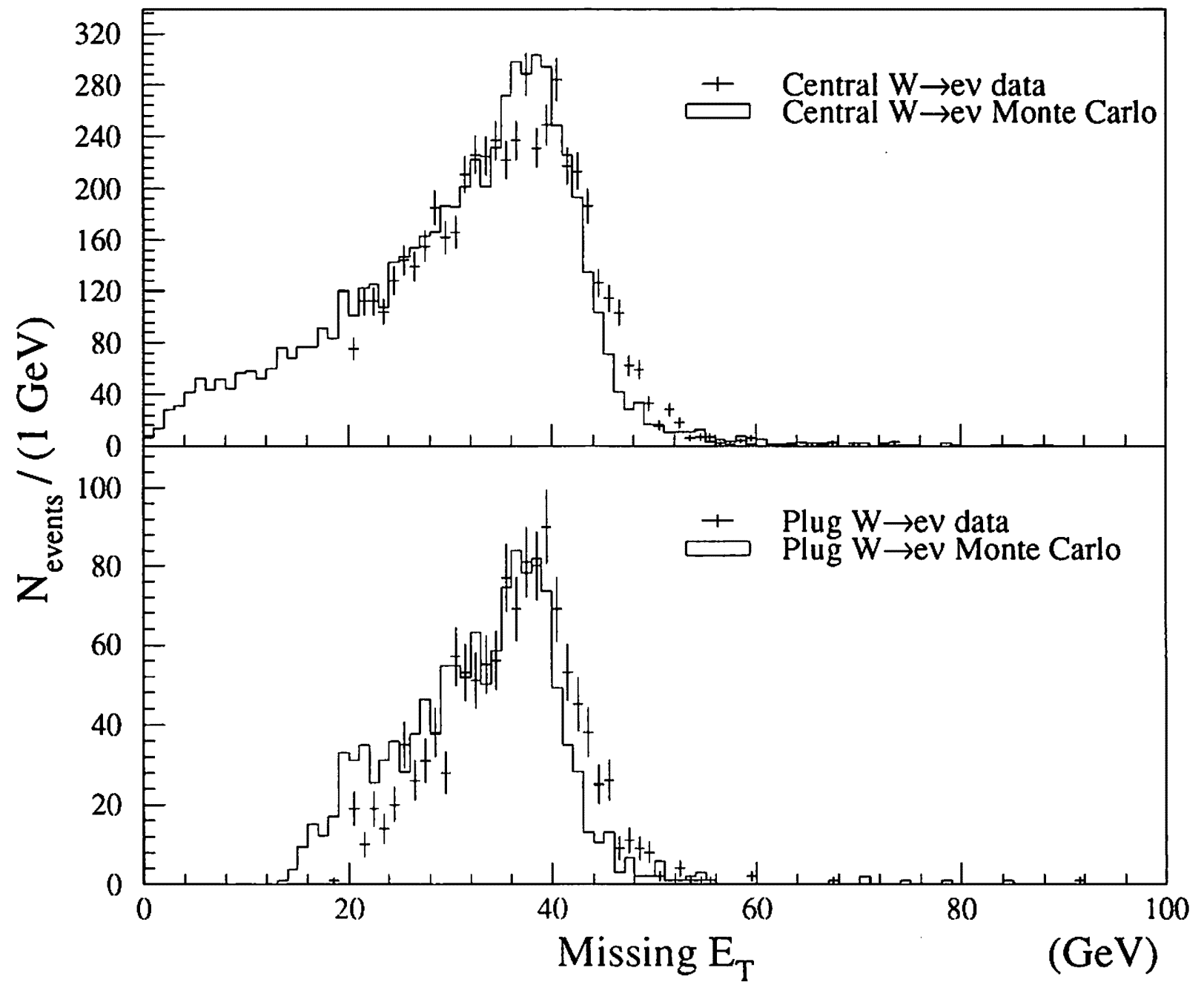

Figure 5.18: Comparison of the $\not_{\mathrm{T}}$ distributions for (a) the CDF central $W \rightarrow e \nu$ data and the Monte Carlo, and (b) the CDF plug $W \rightarrow e \nu$ data and the Monte Carlo. 


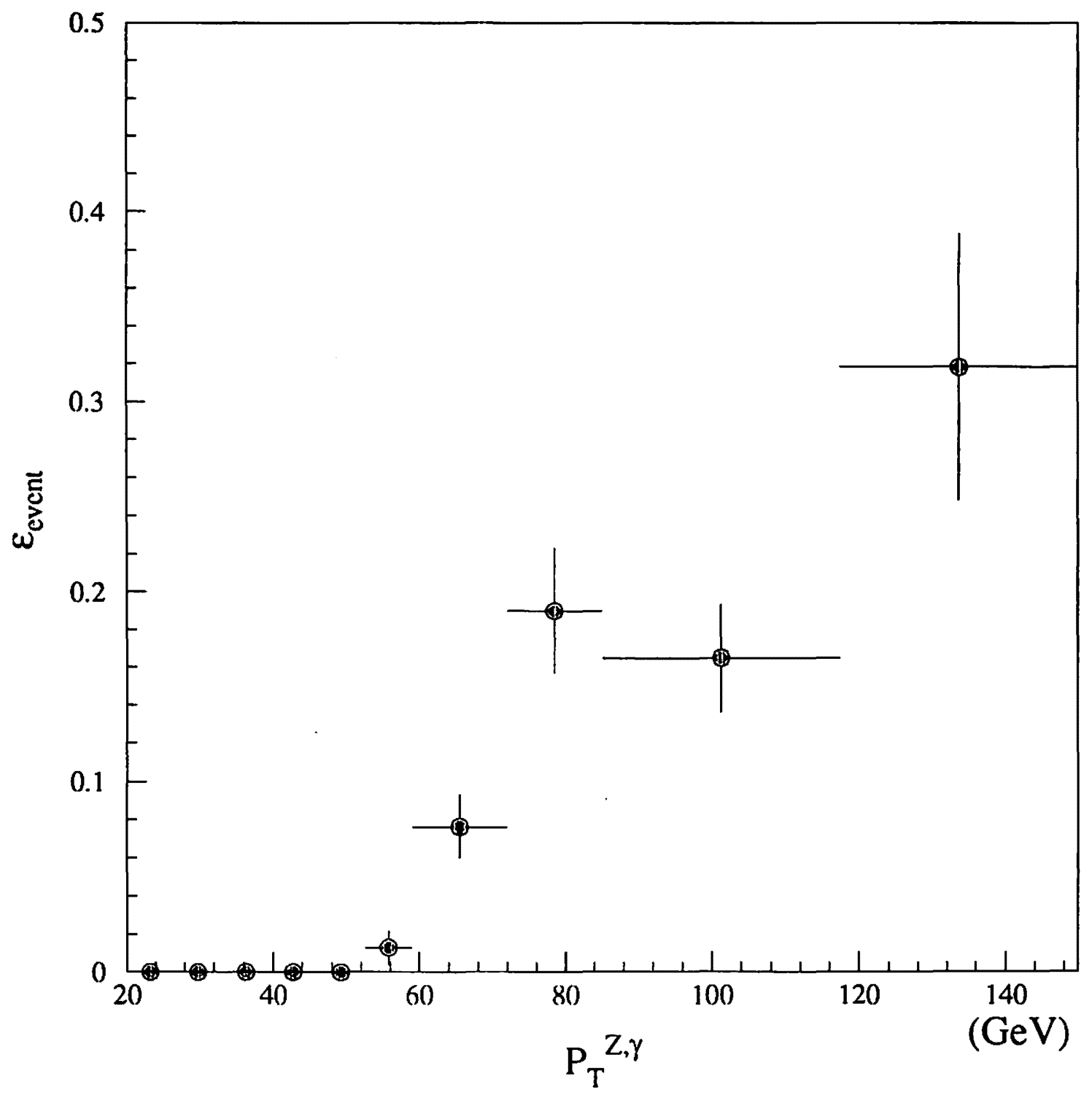

Figure 5.19: The event topology cut efficiency $\varepsilon_{\text {Fivent }}$ for $Z \rightarrow e e, \mu \mu$ Monte Carlo events as a function of $P_{\top}^{Z, \gamma}$. 


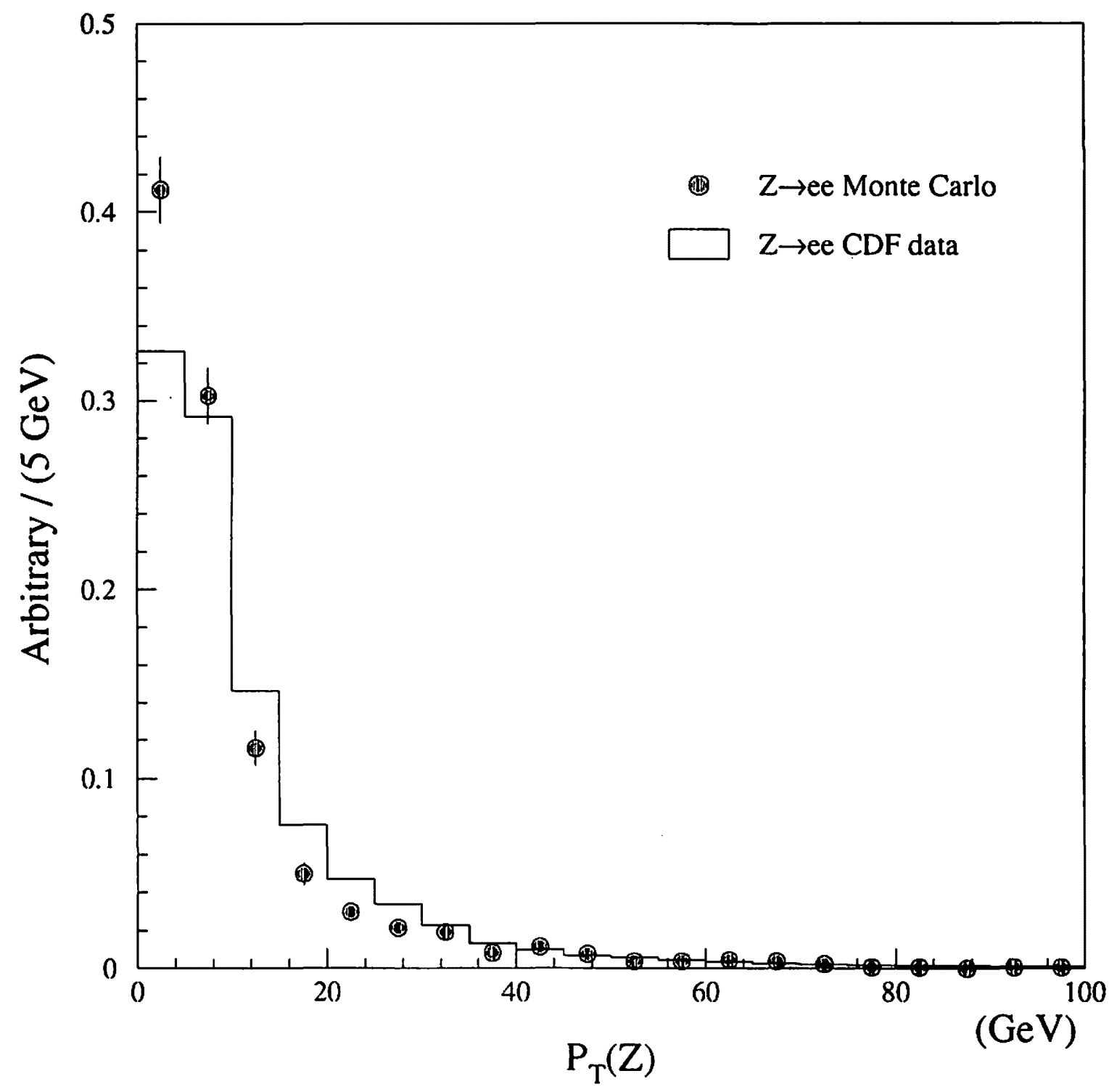

Figure 5.20: The transverse momentum $P_{i}^{\prime}$ distribution from CDF $Z \rightarrow e e$ events. 

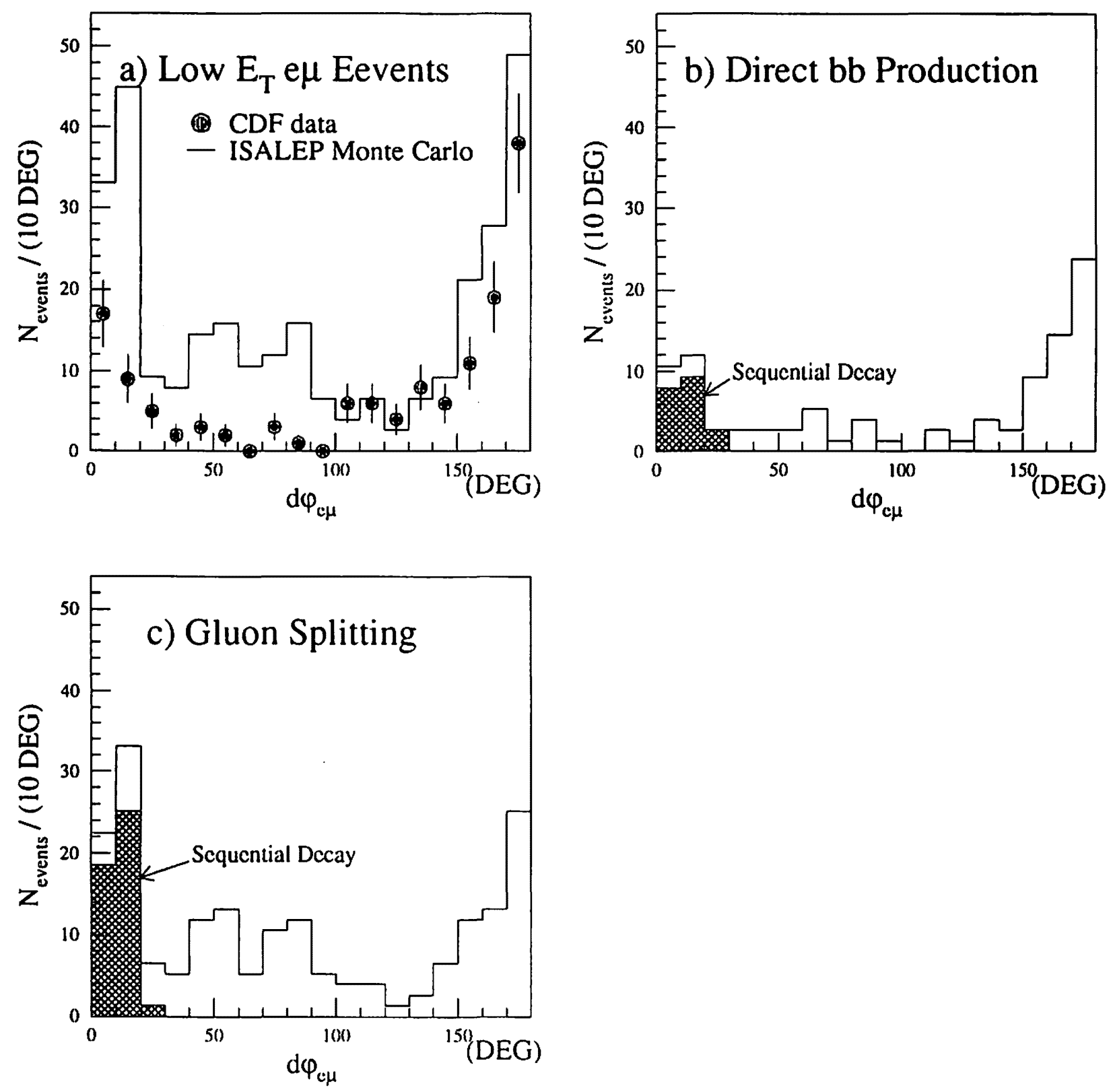

Figure 5.21: The distribution of the azimuthal opening angle, $\Delta \phi_{c \mu}$, between an electron and a muon from low $E_{\uparrow} e \mu$ events. (a) CDF data and ISALEP Monte Carlo prediction. (b) The contribution from $b \bar{b}$ direct production process in the Monte Carlo data. (c) The contribution from gluon splitting process in the Monte Carlo data. 


\section{Chapter 6}

\section{Mass Limit on the Top Quark}

A search described in the previous chapters results in only one candidate event passing our $t \bar{t}$ selection criteria. With one event detected, we can place an upper limit on the number of observed $t \bar{t}$ events, $N_{\text {max }}$, which is translated to an upper limit on the $t \bar{t}$ total production cross section $\sigma(t \bar{t})$ by the equation,

$$
\sigma(t \bar{t})<\frac{N_{\text {trax }}}{L \varepsilon_{\text {lital }}}
$$

where $L$ is the integrated luminosity $\left(4.1 \mathrm{pb}^{-1}\right)$, and $\varepsilon_{\text {Total }}$ is the total detection efficiency for the $t \bar{t}$ events. Using the theoretical prediction for $\sigma(t \bar{t})$ as a function of the top quark mass, we can also derive a limit on the mass of the top quark.

This chapter describes the calculation of the mass limit. In the discussion, another analysis by other CDF collaborators [68] which searches for $t \bar{t}$ events in a different dilepton channel is combined to give the most stringent limit. We refer the analysis described in this thesis and the other independent analysis respectively to

- high $P_{r}$ dilepton (simply "dilepton") analysis

- low Pr muon analysis.

The low $P_{\text {r }}$ muon analysis looks at one high $P_{\text {r }}$ lepton plus jets events. This channel suffers from a large number of $W+j e t s \rightarrow \ell \nu+j e t s$ backgrounds. In order to reduce 
$W+$ jets events, the low $P_{\mathrm{T}}$ muon analysis tags the $b$ quark produced in a $t$ quark decay by detecting the low $P_{\mathrm{r}}$ muon from the decay $b \rightarrow \mu \nu c$. The low $P_{\mathrm{T}}$ muons are required to have $P_{\mathrm{T}}$ less than $15 \mathrm{GeV} / c$. Because of the choice of the $P_{\mathrm{T}}$ cut, this channel is completely separated from the high $P_{\mathrm{r}}$ dilepton channel where muons must have at least $15 \mathrm{GeV} / c$. Therefore we can add its acceptance to the one for the high $P_{\mathrm{T}}$ dilepton channel.

The discussion begins with a calculation of the upper limit on the number of observed events $N_{\text {max }}$ in which the systematic uncertainty discussed in $\S 5.2$ is ignored for clarity. Then we describe how the uncertainty is taken into account in the calculation. Next, the lower mass limit on the top quark from the high $P_{\mathrm{T}}$ dilepton analysis is given. Finally, the more stringent lower limit on the top quark mass is derived by combining the low $P_{\mathrm{T}}$ muon analysis.

\section{Limit Without Systematic Uncertainty}

The number of counts or events found in a large number of experiments is distributed according to the Poisson distribution

$$
P_{\mu}(n)=\frac{\mu^{n} e^{-\mu}}{n !} \quad(n=0,1,2, \ldots)
$$

where the mean $\mu$ is the average number of the observed events related to the cross section by

$$
\mu=L \sigma \varepsilon_{\text {rilal }}
$$

If $n_{0}$ events are observed, the probability that we observe at most $n_{0}$ events is

$$
\alpha=\sum_{n=0}^{n_{0}} P_{n}(n) .
$$

The upper limit on $\mu$ ( $\left.N_{\text {max }}\right)$ can be placed by considering that the observation of $n_{0}$ events is less probable if $\mu>N_{\max }$ which is expressed as a decrease of $\alpha$. Thus the 


\begin{tabular}{|c|cc||c|cc|}
\hline$n_{0}$ & \multicolumn{2}{|c||}{$C L$} & $n_{0}$ & \multicolumn{2}{c|}{$C L$} \\
& $90 \%$ & $95 \%$ & & $90 \%$ & $95 \%$ \\
\hline 0 & 2.30 & 3.00 & 6 & 10.53 & 11.84 \\
1 & 3.89 & 4.74 & 7 & 11.77 & 13.15 \\
2 & 5.32 & 6.30 & 8 & 13.00 & 14.44 \\
3 & 6.68 & 7.75 & 9 & 14.21 & 15.71 \\
4 & 7.99 & 9.15 & 10 & 15.41 & 16.96 \\
5 & 9.72 & 10.51 & & & \\
\hline
\end{tabular}

Table 6.1: Poisson upper limits for $n_{0}$ observed events.

confidence level, $C L$, can be defined as

$$
\begin{aligned}
C L & =1-\alpha \\
& =1-\sum_{n=0}^{n_{n}} P_{\Lambda_{\max }}(n) .
\end{aligned}
$$

In our case $\left(n_{v}=1\right)$, the $95 \%$ C.L. upper limit on the number of $t \bar{t}$ events is obtained by solving the next equation for $N$,

$$
P_{\Lambda^{\prime}}(0)+P_{N}(1)=e^{-N}+N e^{-N^{\prime}}=0.05
$$

The solution is $N_{\max }(95 \% C L)=4.74$. Table 6.1 lists the upper limit numbers for various $n_{0}$ at the $90 \%$ and the $95 \%$ confidence level.

\section{Inclusion of the Systematic Uncertainty}

The systematic uncertainty considered here is the one related to the luminosity measurement and the detection efficiency determination. We consider the uncertainty in the theoretical calculation of the cross section separately from these experimental uncertainties.

The systematic uncertainty in the luminosity measurement and the detection efficiency determination affects the number of observed events. The resulting distribution of the number of observed events over a large number of experiments is no longer a 


\begin{tabular}{|c|cccccc|}
\cline { 3 - 7 } \multicolumn{1}{c|}{} & \multicolumn{7}{c|}{$\sigma / \mu(\%)$} \\
\hline$n_{0}$ & 10 & 11 & 12 & 13 & 14 & 15 \\
\hline 0 & 3.04 & 3.05 & 3.06 & 3.08 & 3.09 & 3.10 \\
1 & 4.84 & 4.86 & 4.88 & 4.90 & 4.93 & 4.96 \\
2 & 6.44 & 6.47 & 6.50 & 6.54 & 6.58 & 6.63 \\
3 & 7.95 & 7.99 & 8.03 & 8.09 & 8.14 & 8.21 \\
4 & 9.40 & 9.45 & 9.51 & 9.58 & 9.65 & 9.73 \\
5 & 10.81 & 10.88 & 10.95 & 11.04 & 11.13 & 11.22 \\
\hline
\end{tabular}

Table 6.2: Upper limits for $n_{0}$ observed events at 95\% C.L. using a Poisson-Gaussian convoluted probability function with the systematic uncertainty of $\sigma / \mu$.

pure Poisson. The modified distribution, $\mathcal{P}_{\mu, \sigma}(n)$, will include a parameter $\sigma$ (this is not a cross section), representing the systematic uncertainty. Preserving the Poisson distribution in base, we take the systematic uncertainty into account as a fluctuation in the average number of events, $\mu$, according to a Gaussian distribution with a standard deviation of $\sigma$,

$$
\begin{aligned}
\mathcal{P}_{\mu, \sigma}(n) & =\int_{0}^{\infty} P_{\xi}(n) G_{\mu, \sigma}(\xi) d \xi \\
G_{\mu, \sigma} & =C e^{-\frac{(\xi-\mu)^{2}}{2 \sigma^{2}}}
\end{aligned}
$$

where the normalization $C$ is chosen so that $\int_{0}^{\infty} G_{\mu, \sigma}(\xi) d \xi=1$, that is, the Gaussian distribution has been truncated so as not to allow negative numbers of events. The systematic uncertainty is assigned to $\sigma$, that is, $\sigma / \mu=13 \%$ in our case. Figure 6.1 compares a Poisson distribution and a modified distribution for $\mu=4.74$ and $\sigma / \mu=13 \%$. As before, the $95 \%$ C.L. upper limit on the number of events for a given value of $\sigma$ is determined by solving for $\mathrm{N}$ the equation,

$$
\mathcal{P}_{N, \sigma}(0)+\mathcal{P}_{N, \sigma}(1)=0.05
$$

Solving Equation (6.9) numerically, we obtain $N_{\max }(95 \% C L)=4.90$ for $\sigma / \mu=13 \%$. Table 6.2 gives the upper limit numbers for various $n_{0}$ and $\sigma / \mu$ at the $95 \%$ confidence level. 


\begin{tabular}{|c|c|c||c|c|}
\cline { 2 - 5 } \multicolumn{1}{c|}{} & \multicolumn{2}{c||}{ dilepton } & \multicolumn{2}{c|}{ dilepton + low $P_{\mathrm{T}} \mu$} \\
\hline $\begin{array}{c}M_{\text {top }} \\
\left(\mathrm{GeV} / c^{2}\right)\end{array}$ & $\begin{array}{c}\varepsilon_{\text {Total }} \\
\left(\times 10^{-1}\right)\end{array}$ & $\begin{array}{c}\sigma_{\iota \grave{l}}(95 \% C L) \\
(\mathrm{pb})\end{array}$ & $\begin{array}{c}\varepsilon_{\text {Total }} \\
\left(\times 10^{-4}\right)\end{array}$ & $\begin{array}{c}\sigma_{\iota \iota}(95 \% C L) \\
(\mathrm{pb})\end{array}$ \\
\hline 70 & 55 & 218 & - & - \\
80 & 68 & 175 & 88 & 136 \\
90 & 80 & 149 & 106 & 113 \\
100 & 83 & 144 & 112 & 107 \\
\hline
\end{tabular}

Table 6.3: The upper limits on the $t \bar{t}$ production cross section from the high $P_{\mathrm{T}}$ dilepton analysis and the combined analysis including the low $P_{\mathrm{T}}$ muon events.

\section{Limit on the Cross Section and the Mass of the Top Quark}

An upper limit on the number of events is converted into an upper limit on the cross section by

$$
\sigma(95 \% C L)=\frac{N_{\text {tnax }}(95 \% C L)}{L \varepsilon_{\text {Tirtal }}}
$$

Table 6.3 lists the total detection efficiency $\left(\varepsilon_{\text {Total }}\right)$ and the upper limit cross section for several top quark masses. The $95 \%$ C.L. upper limit on the $t \bar{t}$ cross section is also shown in Figure 6.2, together with the theoretical calculation of $\sigma(t \bar{t})$. The uncertainty in the theoretical calculation of the $t \bar{t}$ production cross section is indicated as a band in the figure. Conservatively we take the lower limit on the mass of the top quark at $85 \mathrm{GeV} / \mathrm{c}^{2}$ where the upper limit curve intersects the lower edge of the theoretical calculation band.

\section{Inclusion of a Low $P_{\Gamma}$ Muon Analysis}

Table 6.3 also includes the total detection efficiency and the upper limit cross section after combining the low $P_{1}$ muon analysis. The systematic uncertainty in the detection efficiency for the $t \bar{t}$ events in the low $P_{1}$ muon channel combined with the uncertainty in the luminosity measurement is estimated to be $15 \%$. The resulting total systematic uncertainty after the inclusion of the low $P_{\Gamma}$ muon analysis does not change from the total uncertainty obtained in the the high $P_{r}$ dilepton analysis $(13 \%)$ because the contribution of the low $P_{\mathrm{T}}$ muon is relatively small $(\sim 30 \%)$ compared to the high $P_{\mathrm{T}}$ dilepton channel. The combined upper limit cross section curve is shown in Figure 6.2. As a final result, 
the top quark of $M_{\mathrm{top}}<91 \mathrm{GeV} / c^{2}$ is excluded at the $95 \%$ confidence level. 


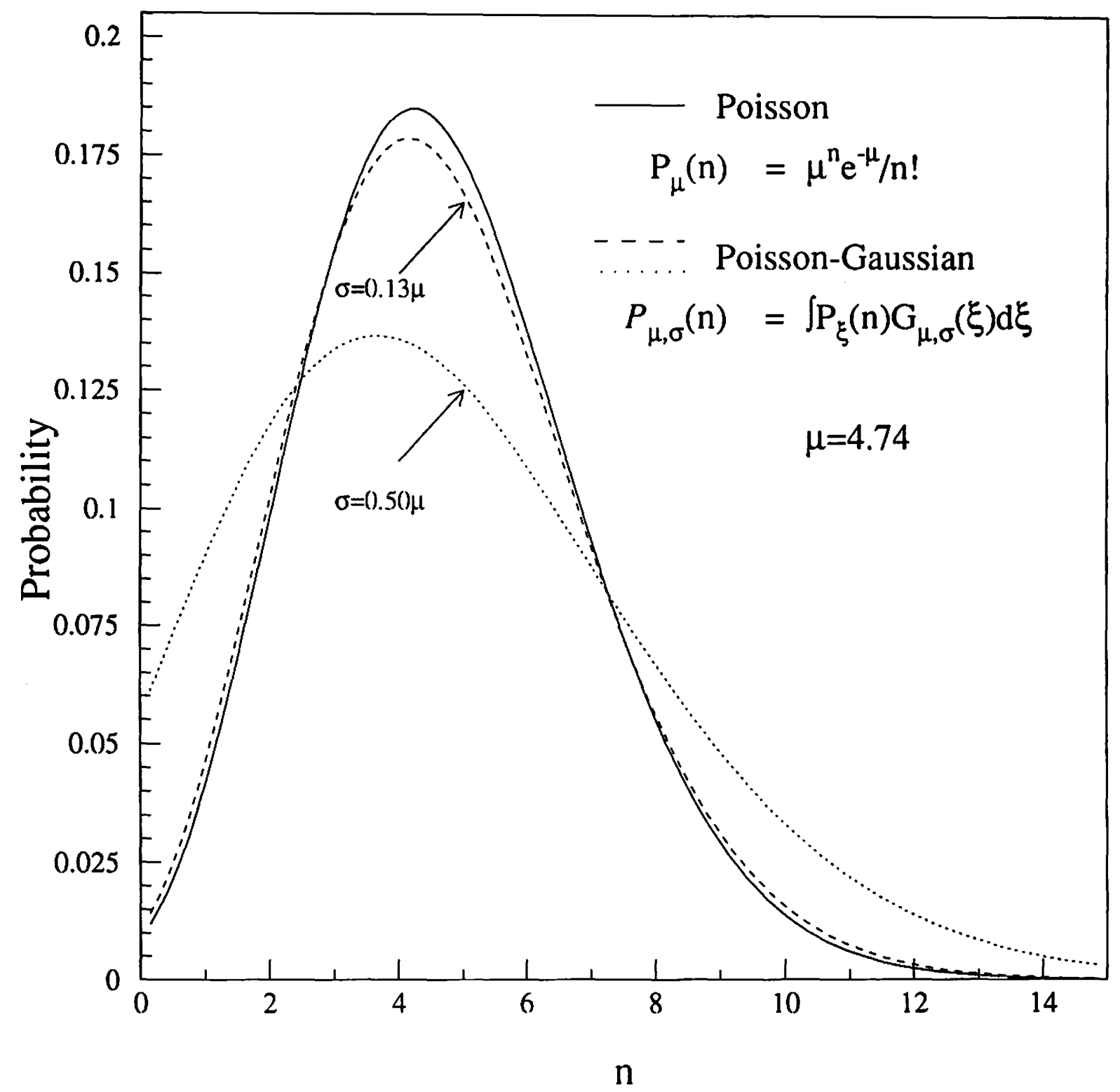

Figure 6.1: A Poisson distribution and a modified distribution (Equation 6.7) for $\mu=$ 4.74. 


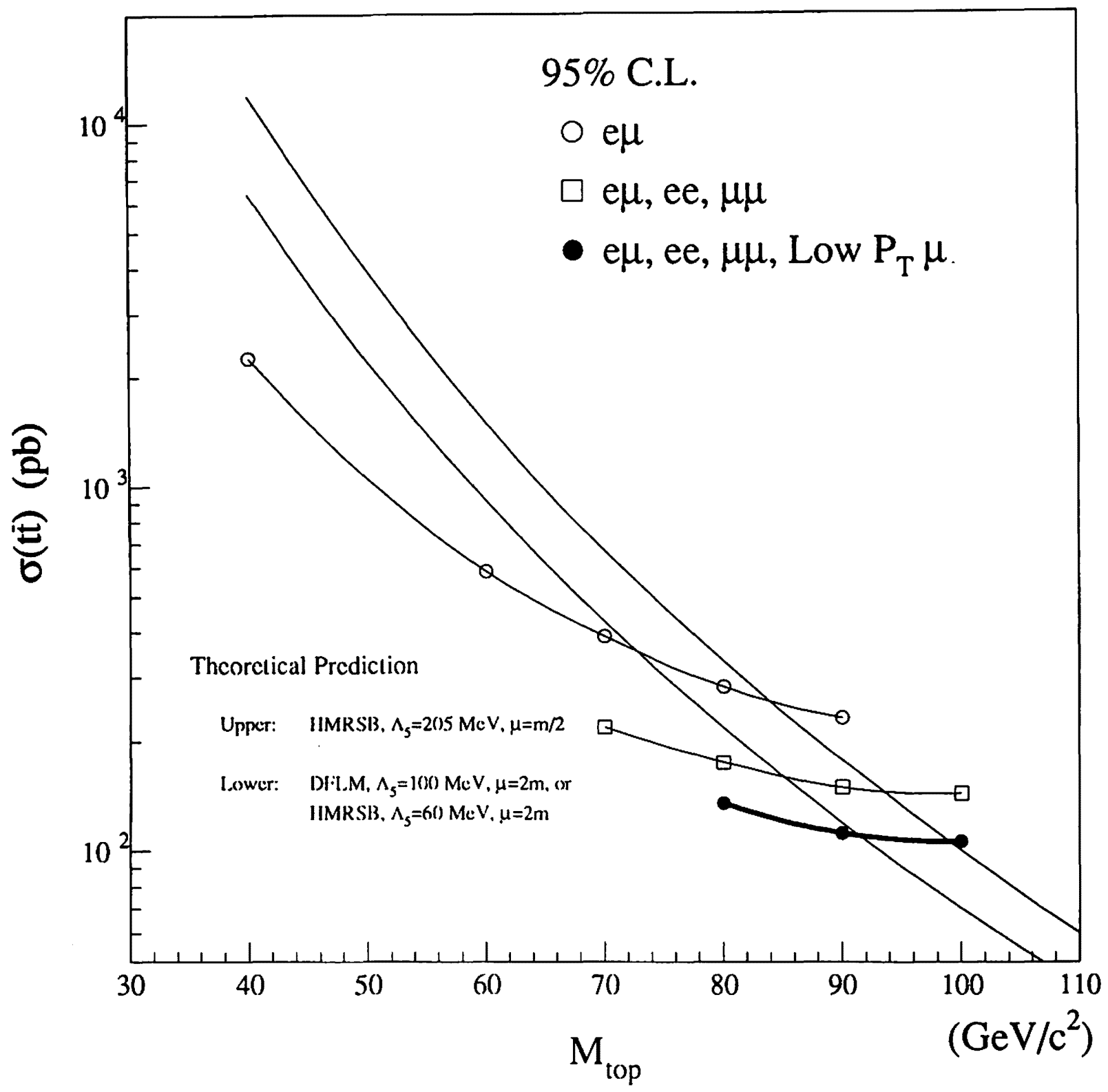

Figure 6.2: The $95 \%$ confidence level limits on $\sigma(t \bar{t})$ compared with a band of theoretical predictions from Reference [24]. We show three sets of experimental limits: (1) From the $e \mu$ analysis of Reference [19], (2) From the analysis of the dilepton modes, (3) From the combination of the dilepton analysis and the low $P_{\mathrm{T}}$ muon analysis. 


\section{Chapter 7}

\section{Conclusions}

We searched for $t \bar{t}$ events in $p \bar{p}$ collisions at $\sqrt{s}=1.8 \mathrm{TeV}$ using the CDF detector. The data were collected in the 1988-1989 CDF run. The corresponding integrated luminosity was $4.1 \mathrm{pb}^{-1}$. We have exploited the good electron and muon identification capabilities of the CDF detector to carry out a search for the top quark in high $P_{\mathrm{T}}$ dilepton events. No decisive evidence for the top quark production has been observed and a lower limit on the top quark mass

$$
M_{\text {Lop }}>85 \mathrm{GeV} / c^{2} \quad(95 \% \text { C.L. })
$$

is obtained. Combining another analysis which searches for $t \bar{t}$ events containing a high $P_{\mathrm{T}}$ lepton plus a low $P_{\text {r }}$ muon from $b$ decay, we obtain

$$
M_{\text {(1) }}>91 \mathrm{GeV} / c^{2} \quad(95 \% \text { C.L. })
$$

as a final result. Currently this is the most stringent limit on the top quark mass available in the world. 


\section{Appendix A}

\section{Luminosity Measurement}

The luminosity measurement at CDF $[69,70]$ is described in this appendix.

The instantaneous luminosity can be obtained directly from the accelerator beam parameters. It is also possible to calculate the luminosity by measuring the rate of a certain process of which the cross section is known,

$$
\mathcal{L}=\frac{R}{\sigma}
$$

where $\mathcal{L}$ is the instantaneous luminosity, $R$ is the rate of the process and $\sigma$ is the cross section for the process. We employed the latter method using the inelastic $p \bar{p}$ interaction cross section at the center of mass energy $(\sqrt{s})$ of $1800 \mathrm{GeV}$ because it gave a more reliable result than the luminosity determined solely from the beam parameters.

At CDF, the inelastic $p \bar{p}$ events are detected with the beam-beam counters (BBC, see $\S 3.1 .1$ ). Given the effective cross section, that is, the cross section visible to the $\mathrm{BBC}$ of the $p \bar{p}$ inelastic scattering, $\sigma_{\mathrm{BBC}}$, and the rate observed by the $\mathrm{BBC}, R_{\mathrm{BBC}}$, the instantaneous luminosity $\mathcal{L}$ is obtained by

$$
\mathcal{L}=\frac{R_{\mathrm{BHC}}}{\sigma_{\mathrm{BHC}}}
$$

We need to know $\sigma_{\mathrm{BBC}}$ at $\sqrt{s}=1800 \mathrm{GeV}$ to calculate the luminosity. 
In earlier publications of the CDF analyses, the luminosity calculation was based on the $\sigma_{\mathrm{BBC}}(1800)$ which was obtained by extrapolating from the SPS collider energy of 546 $\mathrm{GeV}$ to the Tevatron energy [71] assuming the known dependence of the cross section on $\sqrt{s}$. At this time, the difference in the geometry and the efficiency of the trigger counters at CDF and UA4 was corrected using the CDF minimum bias Monte Carlo (MBR) [72]. This method resulted in the total integrated luminosity for the 1988-1989 CDF run of $(4.4 \pm 0.7) \mathrm{pb}^{-1}$.

In order to reduce the uncertainty in the extrapolation, we combined the luminosity determination from the accelerator beam parameters. Since we have collected the data at both $546 \mathrm{GeV}$ and $1800 \mathrm{GeV}$ using the Tevatron, the effective $p \bar{p}$ inelastic cross section at $1800 \mathrm{GeV}$ can be obtained using the equation

$$
\sigma_{\mathrm{BBC}}(1800)=\sigma_{\mathrm{BBC}}(546) \cdot \frac{R_{\mathrm{BHC}}(1800)}{R_{\mathrm{BHC}}(546)} \cdot \frac{\mathcal{L}_{\mathrm{acc}}(546)}{\mathcal{L}_{\text {acc }}(1800)},
$$

where $\sigma_{\mathrm{BBC}}(546)$ is the effective $p \tilde{p}$ inelastic cross section at $546 \mathrm{GeV}$ obtained by converting the measured quantity at $\mathrm{UA} 4, R_{\mathrm{BHC}}$ and $\mathcal{L}_{\text {arc }}$ are the observed $\mathrm{BBC}$ rate and the instantaneous luminosity based on the accelerator parameters at the specified Tevatron energy, respectively. The several uncertainties in the luminosity determination from the beam parameters should cancel out in the ratio of $\mathcal{L}_{\text {acc }}(546)$ and $\mathcal{L}_{\text {acc }}(1800)$, because the beam parameters are measured using the same devices at both energies. From this method, we obtain

$$
\sigma_{\mathrm{BHC}}(1800)=(46.8 \pm 3.2) \mathrm{mb}
$$

The corresponding total integrated luminosity for the 1988-1989 CDF run is

$$
L=\int \mathcal{L} d t=(4.1 \pm 2.8) \mathrm{pb}^{-1}
$$

The fractional uncertainty in the luminosity measurement is reduced from $15 \%$ to $6.8 \%$. 


\section{Bibliography}

[1] S. L. Glashow, Nucl. Phys. 22, 579 (1961).

S. Weinberg, Phys. Rev. Lett. 19, 1264 (1967).

A. Salam, "in Elementary Particle Theory", edited by N. Svartholm (Almqvist and Wiksell, Sweden, 1968), p. 367.

[2] S. L. Glashow, J. Illiopoulos and L. Maiani, Phys. Rev. bf D2, 1285 (1970).

[3] M. Kobayashi and M. Masukawa, Prog. Theor. Phys. 49, 652 (1973).

[4] W. Bartel et al. (JADE collaboration), Phys. Lett. 146B, 437 (1984).

[5] G. L. Kane and M. E. Peskin, Nucl. Phys. B195, 29 (1982).

[6] A. Bean et al. (CLEO collaboration), Phys. Rev. D35, 3533 (1987).

[7] N. Cabbibo, Phys. Rev. Lett. 10, 581 (1963).

[8] J. Ellis et al., Nucl. Phys. B304, 205 (1988).

G. Altarelli et al., Z. Phys. C37, 271 (1988).

[9] F. Abe et al. (CDF collaboration), Phys. Rev. D44, 29 (1991).

[10] D. Bardin et al., Z. Phys. C32, 121 (1986).

[11] G. S. Abrams et al. (MARK II collaboration), Phys. Rev. Lett. 63, 2173 (1989).

D. Decamp et al. (ALEPH collaboration), Phys. Lett. B 231, 519 (1989).

P. Aarnio et al. (DELPHI collaboration), Phys. Lett. B 231, 539 (1989). 
B. Adeva et al. (L3 collaboration), Phys. Lett. B 231, 508 (1989).

M. Z. Akrawy et al. (OPAL collaboration), Phys. Lett. B 231, 530 (1989).

[12] A. D. Martin, W. J. Stirling, and R. G. Roberts, Phys. Lett. B 228, 149 (1989).

[13] U. Amaldi et al., Phys. Rev D36, 1385 (1987).

[14] A. Maki, "Recent Results from TRISTAN", KEK preprint 88-52 (1988).

K. Ogawa, A lecture given at Univ. of Tsukuba (1988).

[15] I. Adachi et al. (TOPAZ collaboration), Phys. Lett. 229, 427 (1989).

[16] D. Decamp et al. (ALEPH collaboration), Phys Lett. 236, 511 (1990).

[17] C. Albajar et al. (UA1 collaboration), Z. Phys. C48, 1 (1990).

[18] T. Åkesson et al. (UA2 collaboration), Z. Phys. C46, 179 (1990).

[19] F. Abe et al. (CDF collaboration), Phys. Rev. Lett. 64, 147 (1990).

[20] F. Abe et al. (CDF collaboration), Phys. Rev. Lett. 64, 142 (1990).

F. Abe et al. (CDF collaboration), Phys. Rev. D43, 664 (1991).

[21] J. C. Collins, D. E. Soper and G. Sterman, Nucl. Phys. B263, 37 (1986).

[22] P. Nason, S. Dawson and R. K. Ellis, Nucl. Phys. B303, 607 (1988).

P. Nason, S. Dawson and R. K. Ellis, Nucl. Phys. B327, 49 (1989). Erratum, Nucl. Phys. B335, 260 (1990).

[23] G. Altarelli, M. Diemoz, G. Martinelli and P. Nason, Nucl. Phys. B308, 724 (1988).

[24] R. K. Ellis, "Rates for top quark production", Fermilab-Pub-91/30-T (1991).

[25] M. Diemoz, F. Ferroni, E. Longo and G. Martinelli, Z. Phys. C39, 21 (1988).

[26] C. Peterson, D. Schlatter, I. Schmitt and P. Z. Zerwas, Phys. Rev. D27, 105 (1983). 
[27] J. Chrin, Z. Phys. C36, 165 (1987).

[28] L. H. Orr, Phys. Rev. D44, 88 (1991).

[29] F. Abe et al., Nucl. Instrum. Methods A271, 387 (1988).

[30] F. Snider et al., Nucl. Instrum. Methods A268, 75 (1988).

[31] F. Bedeschi et al., Nucl. Instrum: Methods A268, 50 (1988).

[32] F. Abe et al., Phys. Rev. Lett. 64, 720 (1989).

[33] L. Balka et al., Nucl. Instrum. Methods A267, 272 (1988).

[34] S. Bertolucci et al., Nucl. Instrum. Methods A267, 301 (1988).

[35] Y. Fukui et al., Nucl. Instrum. Methods A267, 280 (1988).

[36] W. C. Carithers et al., "Performance and Calibration Studies of the C.D.F. Endplug Hadron Calorimeter", CDF internal note 368 (1985).

[37] G. Brandenburg et al., Nucl. Instrum. Method A267, 257 (1988).

S. Cihangir et al., Nucl. Instrum. Method A267, 249 (1988).

[38] G. Ascoli et al., Nucl. Instrum. Method A268, 33 (1988).

[39] M. Contreral, "Search for the Top Quark in Electron-Muon Events in the Collider Detector at Fermilab", PHD Thesis, Brandeis University (1990).

[40] D. Amidei et al., Nucl. Instrum. Method A269, 51 (1988).

[41] E. Barsotti et al., Nucl. Instrum. Method A269, 82 (1988).

T. Carrol et al., Nucl. Instrum. Methods A263, 199 (1988).

[42] G.W. Foster et al., Nucl. Instrum. Method A269, 93 (1988). 
[43] H. Areti et al., Proc. 23rd Int. Conf. on High Energy Physics, Berkeley CA, 1986. I. Gaines et al., Proc. Int. Conf. on Computing in High Energy Physics, Asilomar, 1987 Comput. Phys. Commun 45 (1987) 233.

J. Biel et al., ibid., p331.

[44] E. Barsotti et al., Nucl. Instrum. Methods A269, 82 (1988).

[45] G. Drake et al., Nucl. Instrum. Methods A269, 68 (1988).

[46] H. Brafman et al., IEEE Trans. Nucl. Sci. NS-32, 336 (1985).

[47] E. Barsotti et al., IEEE Trans. Nucl. Sci. NS-32, 1348 (1985).

[48] A. W. Booth et al., Proc. 5th Conf. on Real-Time Computer Applications in Nuclear, Particle and Plasma Physics, San Francisco (1987).

[49] D. Quarrie, B. Troemel, "YBOS Programing Reference Manual", CDF internal note 156 (1988).

[50] J. Hauser, "Guide to the Triggers Used in the 1988-1989 CDF Data Run", CDF internal note 966 (1989).

[51] J. Proudfoot, "A Central Electron Filter for Level 3", CDF internal note 633 (1988).

[52] G. Ascoli et al., Nucl. Instrum. Methods A269, 63 (1988).

A. Gauthier, "Milestones of the Central Muon Trigger", CDF internal note 1029 (1989).

[53] J. Proudfoot, "Electron Identification in the CDF Central Calorimeter", CDF internal note 935 (1989).

[54] CDF Collaboration, F. Abe et al., Phys. Rev. D44, 29 (1991).

[55] CDF Collaboration, F. Abe et al., Phys. Rev. D43, 2070 (1991). 
[56] S. Errede, W. Trischuk, "E/P Calibration of the CEM Calorimeter", CDF internal note 1081 (1990).

[57] R. M. Harris, "Definition of CES $\chi^{2 ",}$ CDF internal note 1329.

[58] K. Ragan, J. Walsh and H. Williams, "Identification of Photon Conversions", CDF internal note 902 (1989).

M. Gold, "Identification of Conversion Electron Pairs", CDF internal note 913 (1989).

[59] F. E. Paige and S. D. Protopopescu, "ISAJET 5.20: A Monte Carlo event generator for $p p$ and $p \bar{p}$ interactions.", BNL Report No. BNL-38034, 1986 (unpublishied).

[60] J. Freeman, "CDF detector simulation", Proceedings of the Workshop on Detector Simulation for the SSC, Argonne, I.L., August 1987, edited by L. E. Price, Argonne National Laboratory, p. 190.

[61] T. Westhusing, "Observation of $W \rightarrow \mu \nu$ decays in Proton-Antiproton Collisions at $\sqrt{s}=1.8 \mathrm{TeV} "$, PHD Thesis, University of Illinois at Urbana Champaign (1989).

[62] A. Byon-Wagner et al., "Cosmic filtering of central muon events", CDF internal note $1260(1990)$.

[63] J. Proudfoot, " Central Electron $12 \mathrm{GeV}$ Trigger Efficiency", CDF internal note 1008 (1989).

[64] M. Contreras, Y. Seiya and G. P. Yeh, "Dilepton Analysis for Top Search including Plug Electrons", CDF internal note 1351 (1991).

[65] A. Gauthier, "Efficiency of the level 1 central muon trigger", CDF internal note 937 (1989).

A. Gauthier et al., "Efficiency of the level 2 central muon trigger", CDF internal note 1106 (1990). 
A. Gauthier, "Efficiency of the level 3 central muon trigger", CDF internal note 1145 (1990).

[66] I. Hinchliffe, Private Communication.

[67] ISALEP is a special version of ISAJET which is modified to generate heavy quark events with high- $P_{\mathrm{T}}$ leptons effectively.

[68] C. Campagnari, P. Tipton, "Search for Low $P_{\Gamma}$ muons for Top Decay", CDF internal note 1122 (1990).

C. Campagnari, P. Tipton, "What's new in the Low $P_{\mathrm{T}}$ Muon Analysis (Version 2.1)", CDF internal note 1425 (1990).

[69] C. Grosso-Pilcher et al. (CDF collaboration), "CDF luminosity calibration", Preprint Fermilab-FN-550 (1990).

[70] F. Abe et al. (CDF collaboration), Phys. Rev. D44, 29 (1991).

[71] T. Liss, "Luminosity Monitoring and Beam-Beam Counter Performance", CDF internal note 552 .

[72] S. Belforte and K. Goulianos, "A complete minimum bias event generator", CDF internal note 256 (1984).

[73] M. Bozzo et al. (UA4 collaboration), Phys. Lett. B147, 392 (1984).

D. Bernard et al. (UA4 collaboration), Phys. Lett. B198, 583 (1987). 

$\frac{1}{20}$

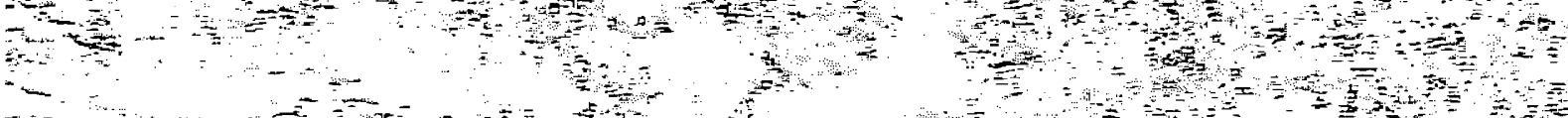

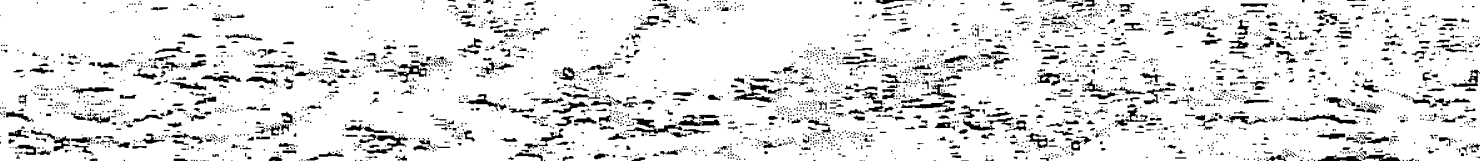
$+1$

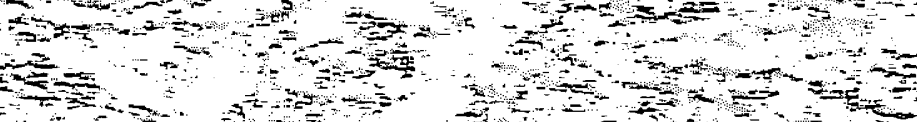

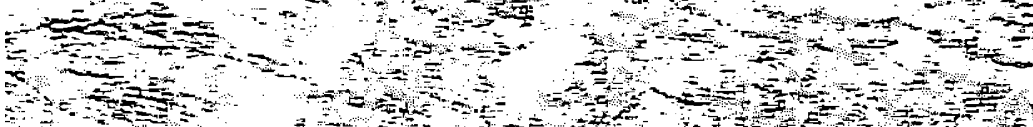
Fond,

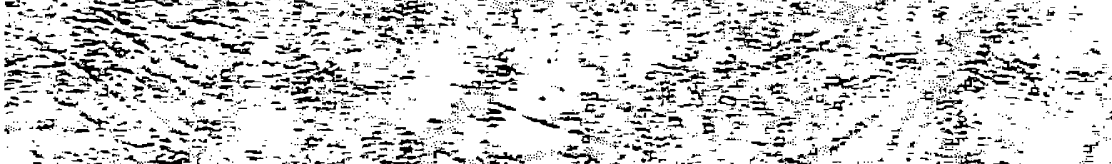

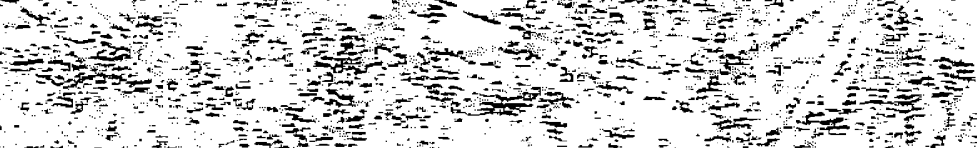

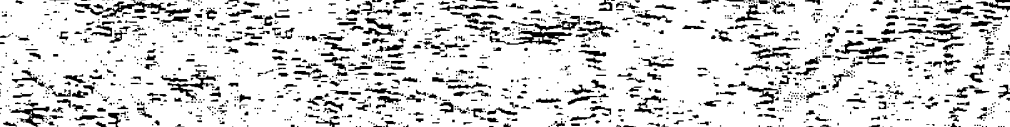
E

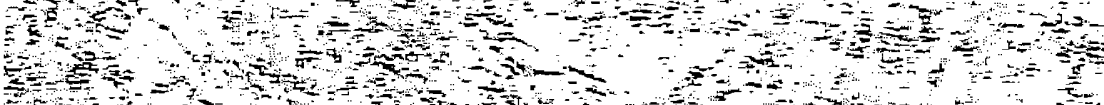

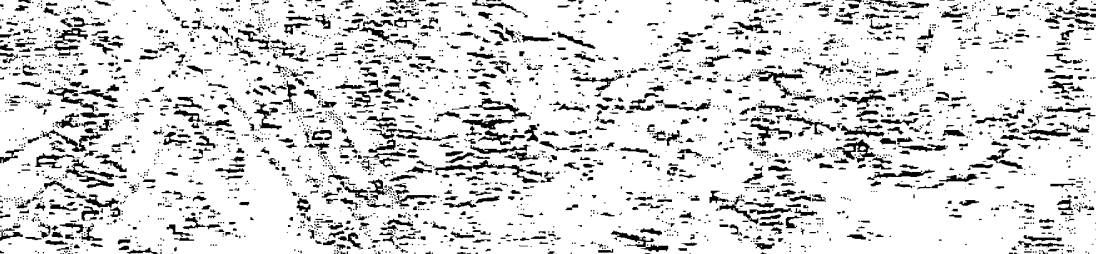

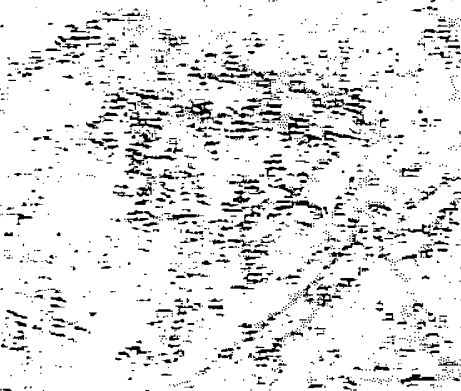

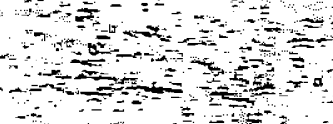

3

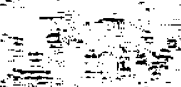

$+3$

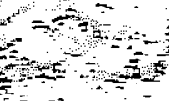

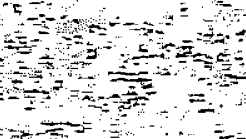

s.

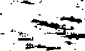

mint

8

tote

$=8+2$

$+2$

tos

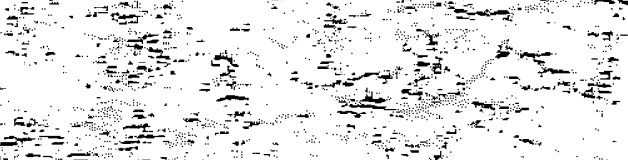

$x_{=1}$

$+3$

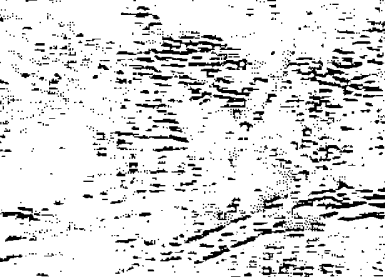

$2-2-2=0$

$-1$

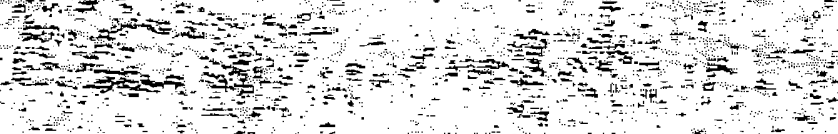

tis.

$+2$

and

$+2=0$

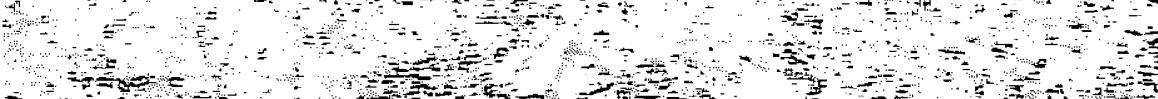

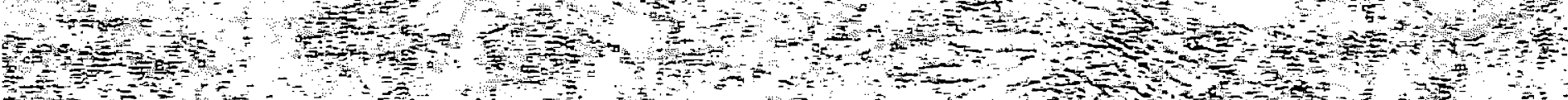

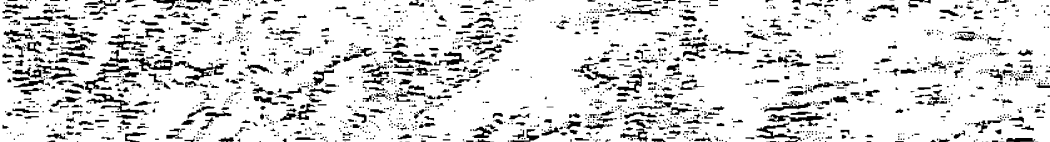

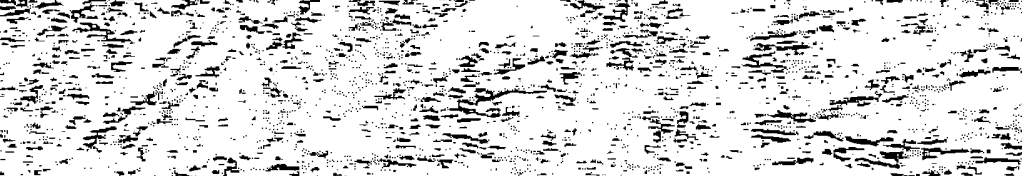
$=0$

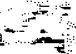
3

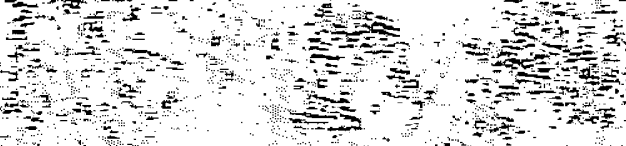
$7+5$

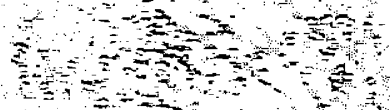

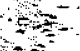

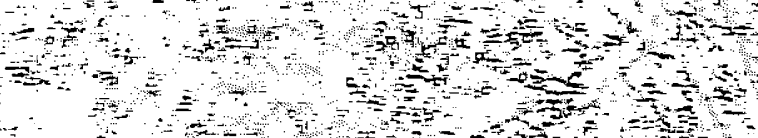

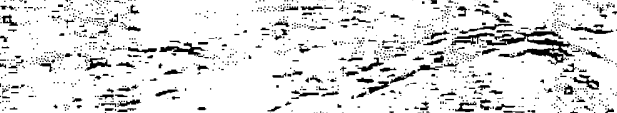

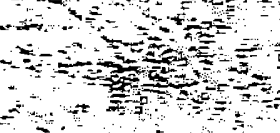
$+1$

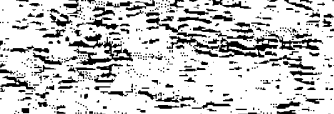

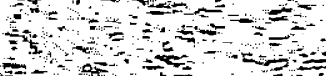

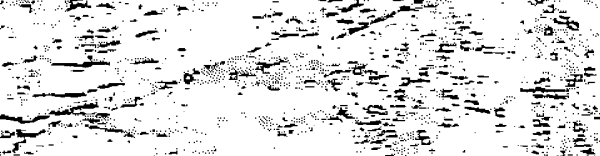

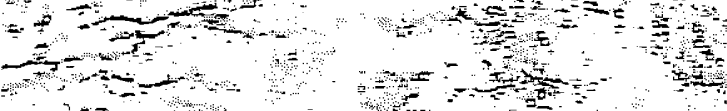

$n_{2}$

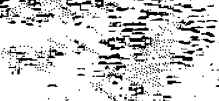

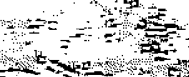

$=y^{2}$

$3=$

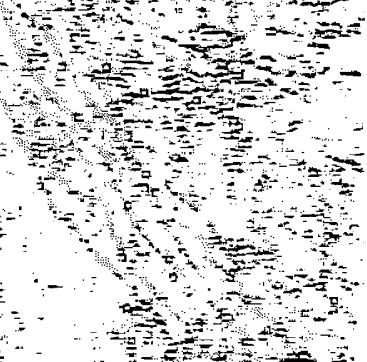

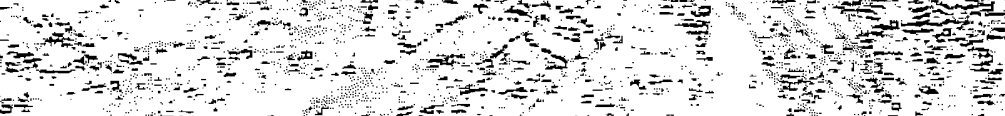

$+2$

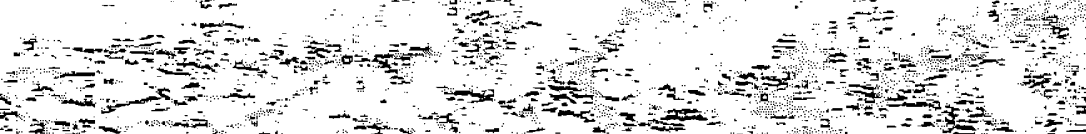

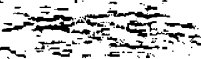

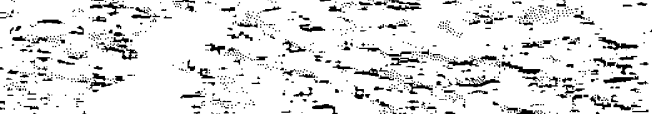

